What Fundamental Properties Suffice

to Account for the Manifest World?

Powerful Structure

Sharon Rose Ford

A thesis submitted for the degree of Doctor of Philosophy at

The University of Queensland in January, 2010

School of History, Philosophy, Religion and Classics 


\section{Declaration by author}

This thesis is composed of my original work, and contains no material previously published or written by another person except where due reference has been made in the text. I have clearly stated the contribution by others to jointly-authored works that I have included in my thesis.

I have clearly stated the contribution of others to my thesis as a whole, including statistical assistance, survey design, data analysis, significant technical procedures, professional editorial advice, and any other original research work used or reported in my thesis. The content of my thesis is the result of work I have carried out since the commencement of my research higher degree candidature and does not include a substantial part of work that has been submitted to qualify for the award of any other degree or diploma in any university or other tertiary institution. I have clearly stated which parts of my thesis, if any, have been submitted to qualify for another award.

I acknowledge that an electronic copy of my thesis must be lodged with the University Library and, subject to the General Award Rules of The University of Queensland, immediately made available for research and study in accordance with the Copyright Act 1968.

I acknowledge that copyright of all material contained in my thesis resides with the copyright holder(s) of that material.

\section{Statement of Contributions to Jointly Authored Works Contained in the Thesis}

No jointly-authored works.

\section{Statement of Contributions by Others to the Thesis as a Whole}

No contributions by others.

\section{Statement of Parts of the Thesis Submitted to Qualify for the Award of Another Degree} None.

\section{Published Works by the Author Incorporated into the Thesis}

Ford, S. (2007). An Analysis of Properties in John Heil's 'From an Ontological Point of View'. In G. Romano (Ed.), Symposium: From an Ontological Point of View, Vol. 6, (pp. 42-51). Philosophy of Mind Review: SWIF http://gxserver.uniba.it/mind/swifpmr/0620072.pdf - sections of author postprint revised and edited for inclusion in Chapter 11.

Ford, S. (Forthcoming). 'The Categorical-Dispositional Distinction'. In, Issues in the Metaphysics of Scientific Realism. - sections of author postprint revised and edited for inclusion Chapter 7.

\section{Additional Published Works by the Author Relevant to the Thesis but not Forming Part of it} None. 


\section{Acknowledgements}

I wish to thank my Advisory team, Phil Dowe and Dominic Hyde, for their time, energy and guidance, provided throughout the course of this research project. I also thank David Armstrong and Brian Ellis, who generously furnished feedback on drafts of certain chapters. I would also like to thank the Philosophy community at the University of Queensland for their camaraderie and support, for the seminars and symposiums, and for their being a family to me over the course of my studies. I also owe a great debt of gratitude to Merin Nielsen for his tireless engagement in conversing about the topics contained herein. Many of the ideas and arguments that feature in this Thesis were refined in the course of those fruitful, and sometimes energetic, conversations. I also credit him for his wonderful patience, and for suffering without complaint, my frequent status as 'otherwise engaged'. I would also like to thank my son, Thomas, for his patience and support. He has never known a time when his mother was not either studying, researching or writing. 


\section{$\underline{\text { Abstract }}$}

This Thesis engages with contemporary philosophical controversies about the nature of dispositional properties or powers and the relationship they have to their nondispositional counterparts. The focus concerns fundamentality. In particular, I seek to answer the question, 'What fundamental properties suffice to account for the manifest world?' The answer I defend is that fundamental categorical properties need not be invoked in order to derive a viable explanation for the manifest world. My stance is a field-theoretic view which describes the world as a single system comprised of pure power, and involves the further contention that 'pure power' should not be interpreted as 'purely dispositional', if dispositionality means potentiality, possibility or otherwise unmanifested power or ability bestowed upon some bearer.

The theoretical positions examined include David Armstrong's Categoricalism, Sydney Shoemaker's Causal Theory of Properties, Brian Ellis's New Essentialism, Ullin Place's Conceptualism, Charles Martin's and John Heil's Identity Theory of Properties and Rom Harré's Theory of Causal Powers. The central concern of this Thesis is to examine reasons for holding a pure-power theory, and to defend such a stance. This involves two tasks. The first requires explaining what plays the substance role in a pure-power world. This Thesis argues that fundamental power, although not categorical, can be considered ontologically-robust and thus able to fulfil the substance role. A second task-answering the challenge put forward by Richard Swinburne and thereafter replicated in various neo-Swinburne arguments-concerns how the manifestly qualitative world can be explained starting from a pure-power base. The Light-like Network Account is put forward in an attempt to show how the manifest world can be derived from fundamental pure power.

\section{$\underline{\text { Keywords }}$}

dispositional properties, categorical properties, Foundation-Monism, light-like network, categoricalism, causal theory of properties, new essentialism, conceptualism, identity theory of properties, Harré

\section{Australian and New Zealand Standard Research Classifications (ANZSRC)}

220309 Metaphysics 


\title{
What Fundamental Properties Suffice \\ to Account for the Manifest World? \\ Powerful Structure
}

\author{
Sharon Ford \\ University of Queensland
}

\section{TABLE OF CONTENTS}

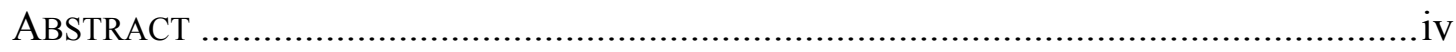

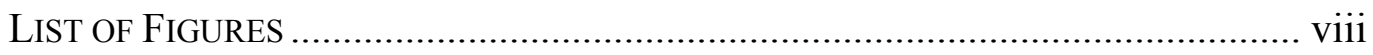

LIST OF ABBREVIATIONS............................................................................... viii

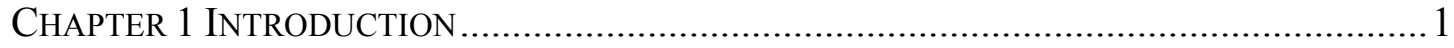

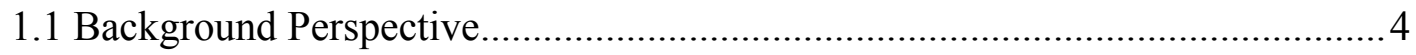

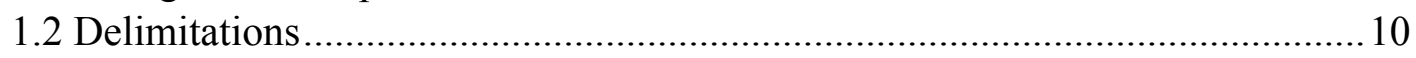

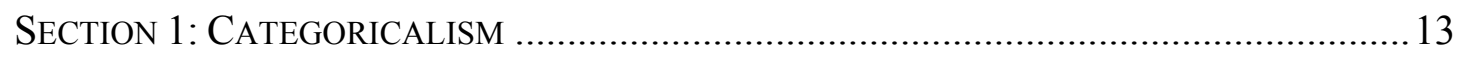

Chapter 2 DAVID ARMSTRONG: CATEGORICALISM - OutLINE .................................... 15

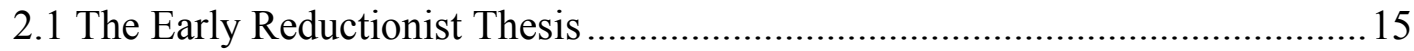

2.2 From Metaphysical Reduction to a Supervenient Thesis of Dispositions ......... 17

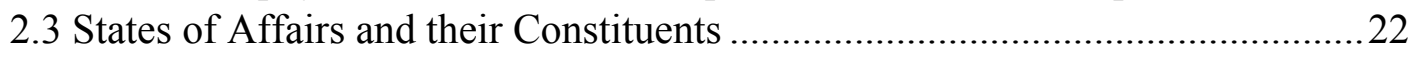

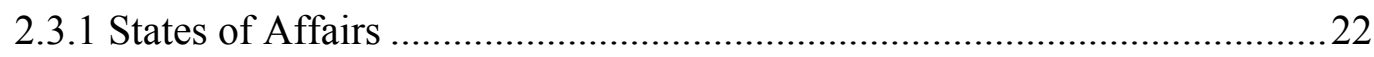

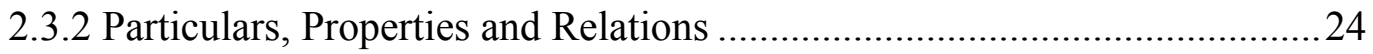

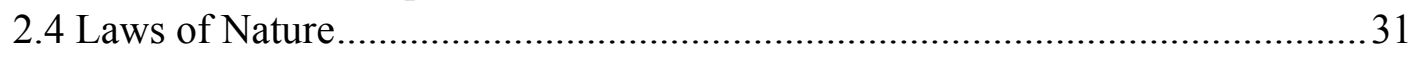

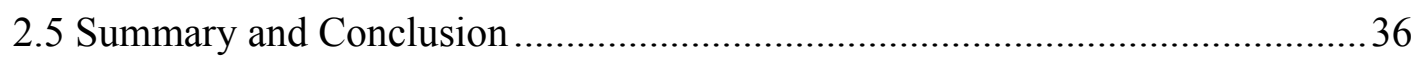

Chapter 3 DAVID ARMSTRong: CATEGORICALISM - DisCUSSION................................. 37

3.1 Martin's Argument Concerning Connectability and Repeatability ....................40

3.2 Hochberg's Criticism of N: Reliance on Ambiguity ......................................4

3.3 Bird's Formal Characterisation................................................................ 43

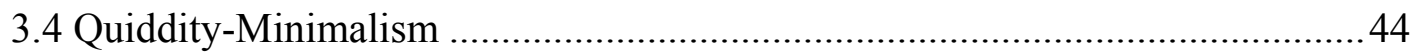

3.5 Revisions Concerning Instantiation and Laws ............................................ 47

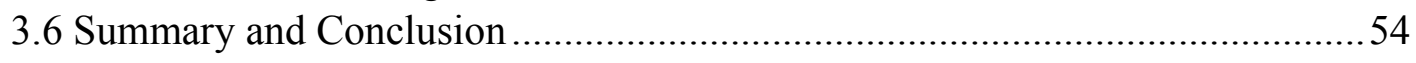

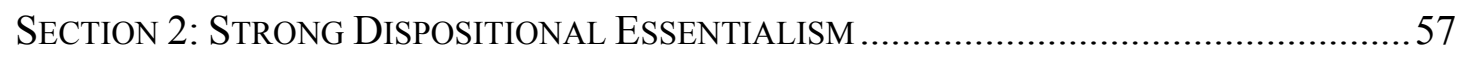

Chapter 4 Sydney Shoemaker: Causal Theory of Properties - Outline .........59

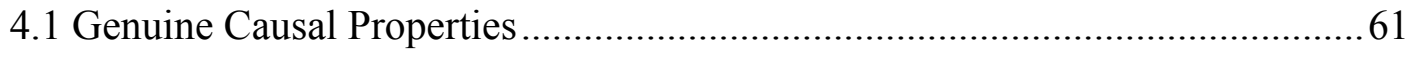

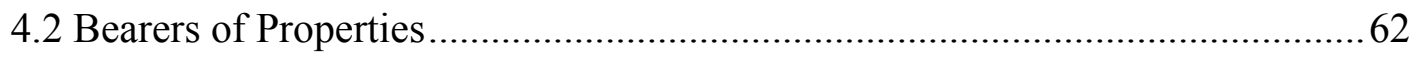




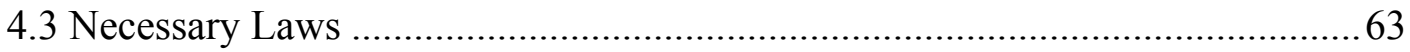

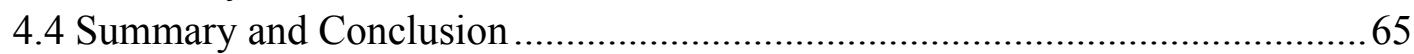

Chapter 5 Sydney SHOEMAKer: CAUSAL TheOry of Properties - Discussion .... 67

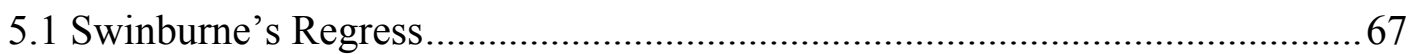

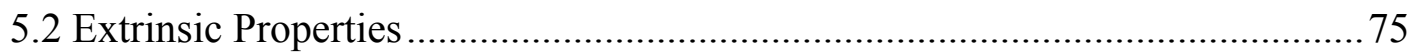

5.2.1 Argument 1: Molnar's Argument Against Foundationism........................ 76

5.2.2 Argument 2: Relational Properties, Although Not Powers, are Causally

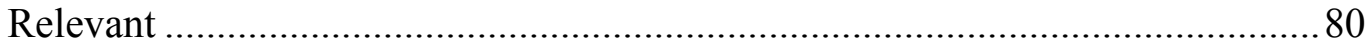

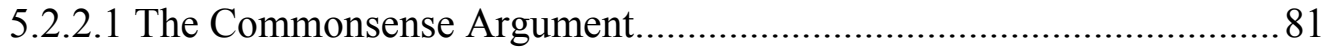

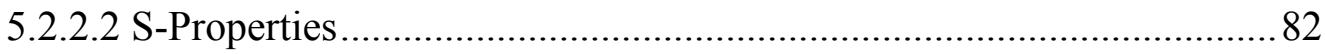

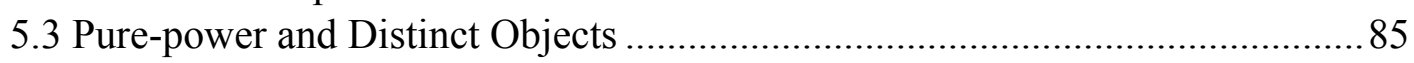

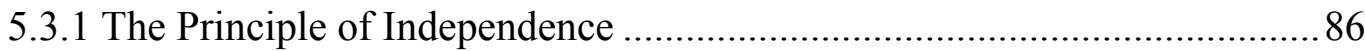

5.3.2 Specifying Circumstances for Power Manifestation................................... 88

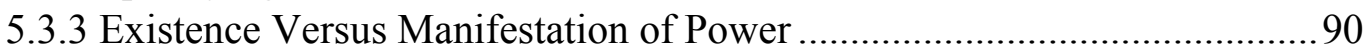

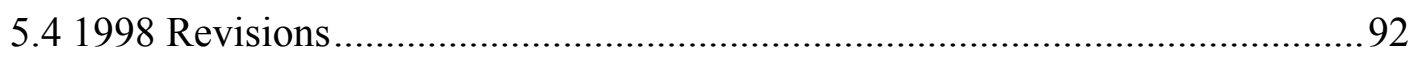

5.4.1 Properties Possessing Essential Causal Features ........................................ 93

5.4.2 Properties No longer Clusters of Conditional Power..................................94 94

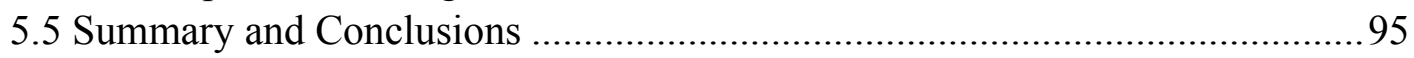

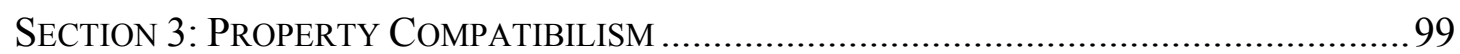

Chapter 6 Brian ElLis: New EsSEnTIALISM - OutLINE …................................... 101

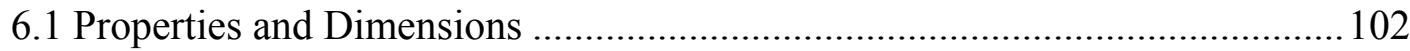

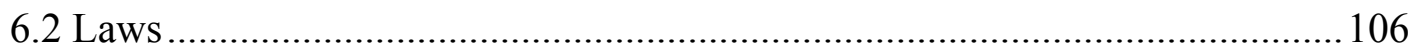

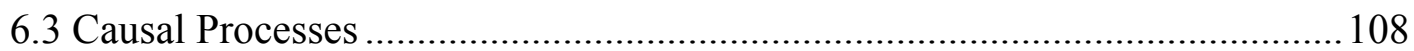

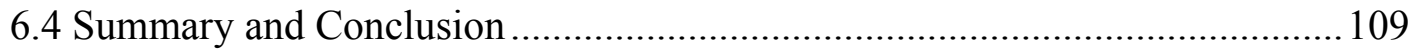

CHAPTER 7 BRIAN ELLIS: NEW ESSENTIALISM - DiSCUSSION .................................... 111

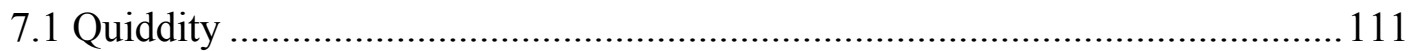

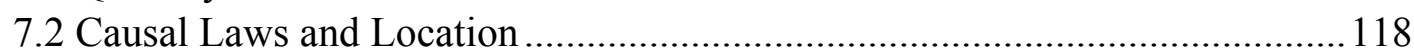

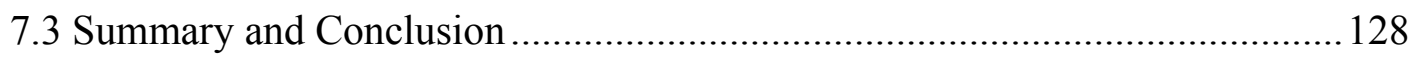

ChaPTER 8 Ullin Place: ConcePtUAlism - OUtLINE ........................................... 131

Chapter 9 Ullin Place: ConcePtualism - Discussion ........................................ 137

9.1 Truthmakers for Dispositional Properties ...................................................... 137

9.2 The Causal Role of the Microstructure ….................................................... 140

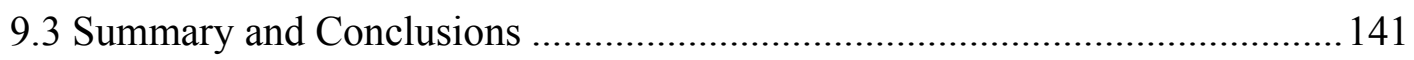

Chapter 10 Charlie Martin \& John Heil: IDENTITy Theory of Properties -

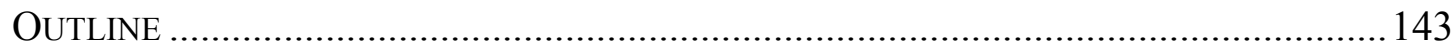

10.1 Two Irreducible but Inseparable Natures...................................................... 147

10.2 The Dispositional and Qualitative are a Single Nature............................... 150

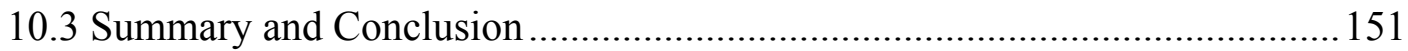


CHAPTER 11 CHARLIE MARTIN \& JOHN HeIL: IDENTITY THEORY OF PROPERTIES -

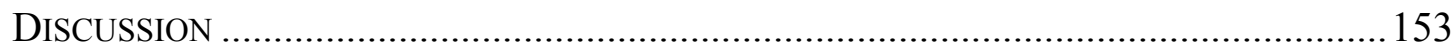

11.1 Differently Considering the Dispositional and Qualitative ........................... 155

11..2 Non-relational Dispositional Properties.................................................... 157

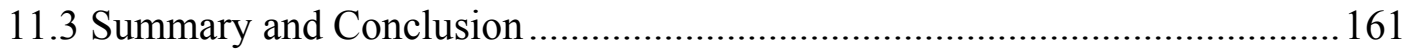

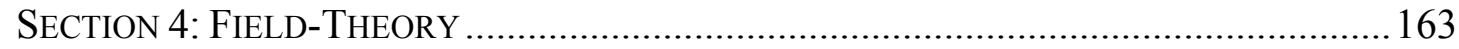

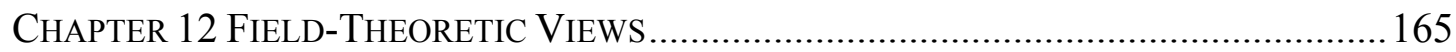

12.1 Field-Theoretic View of Keith Campbell .................................................... 168

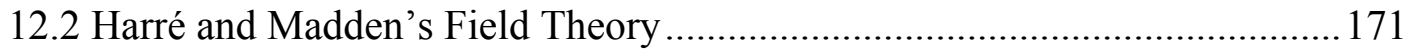

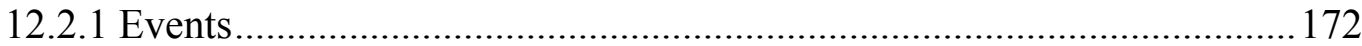

12.2.2 Laws of Nature and Generative Mechanisms; Distinguishing Causal from

Accidental Sequences ........................................................................ 178

12.2.3 Generative Mechanisms, Explanatory Regress and Parmenidean

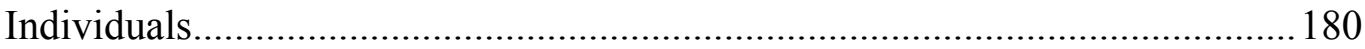

12.2.4 Roger Boscovich - Law of Continuity ............................................... 183

12.2.5 Harré's Field Theory of Power ........................................................... 186

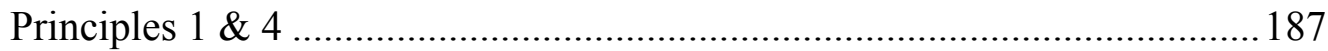

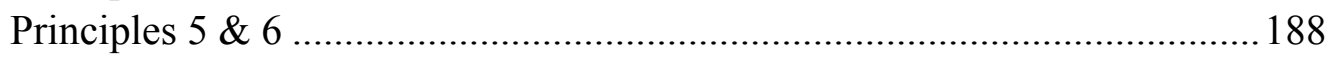

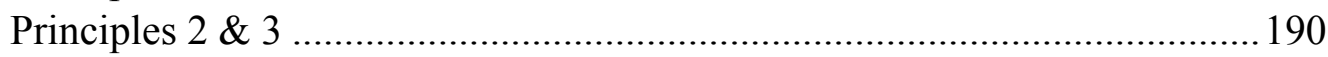

12.2.6 Questioning the Ontological Priority of Spacetime ............................... 193

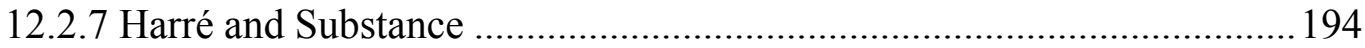

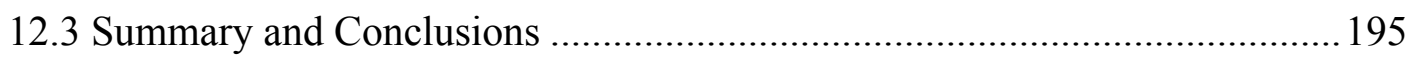

Chapter 13 The Substance Role in a Pure-Power World .................................. 197

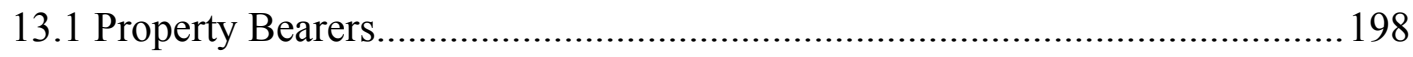

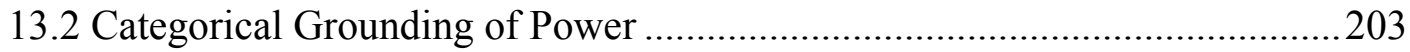

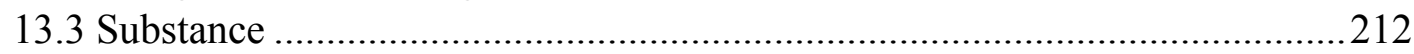

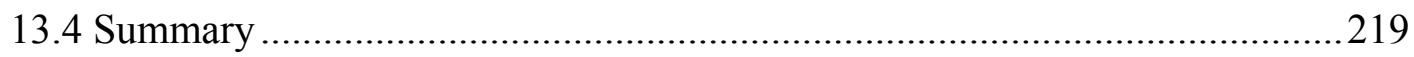

Chapter 14 Deriving the Manifestly Qualitative World from a Pure-Power

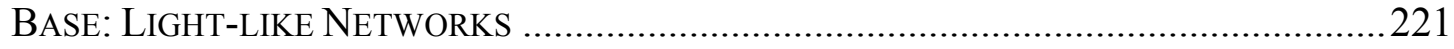

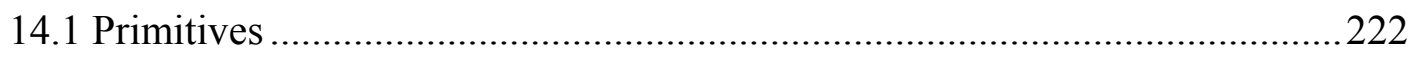

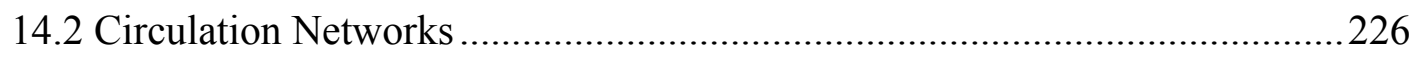

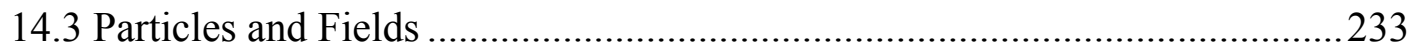

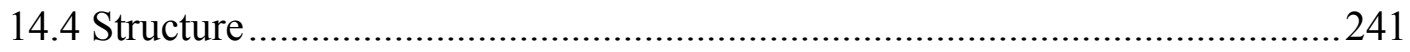

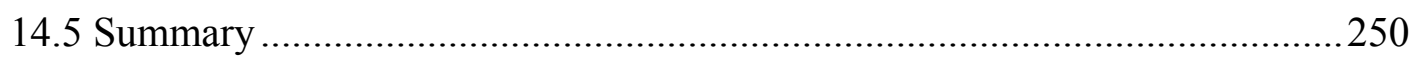

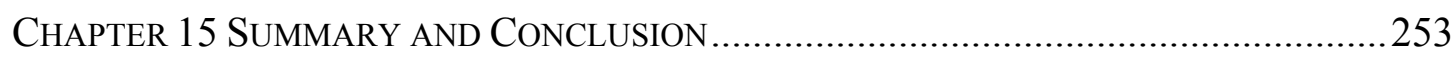

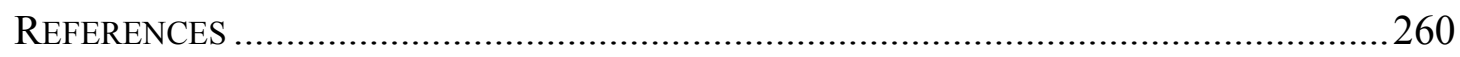

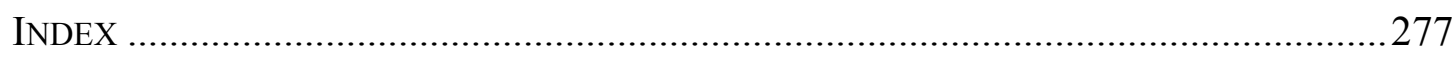




\section{LIST OF FIGURES}

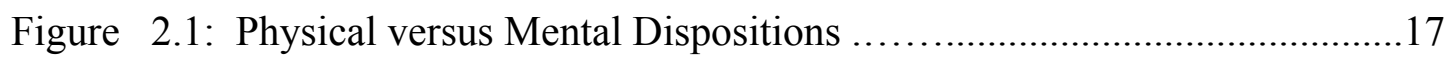

Figure 14.1: 'Barber Pole’ Compared With Gauge Boson Path ..........................229

Figure 14.2: Network Trajectories of Gauge Bosons...............................230

\section{List of AbBREviations}

$\mathrm{N}$ - Necessitation Relation

DE - Dispositional Essentialism

$\mathrm{TI}$ - transcendental individuality

LQG - Loop Quantum Gravity

PII - Principle of the Identity of Indiscernibles

LNA - Light-like Network Account

QFT - Quantum Field Theory 


\section{CHAPTER 1}

\section{INTRODUCTION}

This Thesis focuses on what kinds of properties are required at the fundamental level to provide a satisfactory explanation for the manifest world. Traditionally, a sharp divide has been imposed between dispositional and categorical properties, the two often being defined in mutually exclusive and oppositional terms. Categorical and/or qualitative properties have been typically described as involving spatial extension or space-occupation, and denoted by primary Lockean properties such as size, shape, solidity and so on (Locke,, II, Ch. VIII, p. 66). Brian Ellis notes that categorical properties have been considered readily imaginable (2002, p. 68); existing independently of behaviour (pp. 68-69); multi-dimensional (p. 69); structural (pp. 6970); non-dispositional (pp. 70, 117); and the ground or realiser of the dispositional (pp. 174-175). They have been described by Ullin Place as having an 'actual' or ontologically-robust status (1996b), and by David Armstrong as being self-contained in terms of 'completeness' in their instantiation. Armstrong describes their nature as 'exhausted' in their instantiation by particulars, whereby they do not reserve of themselves for further interactions with other particulars (1989, p. 118; 1997, pp. 41, 69, 245). Alexander Bird describes them as properties that have primitive identity (2007, p. 45). Charlie Martin describes his version of categorical propertiesqualitative properties - as those needed for things to be perceived, providing the 'what' or 'shell' of objects (1997), and John Heil describes them as what individuates or differentiates powers $(2007$, p. 84$){ }^{1}$

Dispositional properties have often been contrasted with categorical properties in all of the descriptive contexts above. ${ }^{2}$ As Ellis points out, dispositional properties have been considered: uni-dimensional (2002, p. 69-70); essentially modal (p. 70); and grounding the categorical (pp. 174-175). One traditional characterisation of a 'disposition' portrays it as that which determines how something will tend to react under different kinds of circumstances. Paradigmatic examples include brittleness or

\footnotetext{
1 The subtle differences between Martin and Heil's qualitative properties and their categorical counterparts will be discussed in more detail in Chapter 9.

${ }^{2}$ The emphasis in this paper concerns the metaphysical difference between the two, rather than a merely predicative differentiation.
} 
solubility. Dispositions are often expressed using the form: if $p$ were the case, then $q$ would be the case. To say, for example, that a substance has the disposition of solubility, is to say that ceteris paribus if it is placed in certain kinds of liquids at certain temperatures then it will dissolve.

Some philosophers use the terms 'disposition' and 'dispositional property' interchangeably. Armstrong, for example, views a disposition as a property that can be possessed by something; and, referring to the ability to effectively interact either actively or passively, he uses the term interchangeably with power. ${ }^{3}$ Ellis, however, asserts that 'dispositional properties' cannot be defined in behavioural terms in the same way we talk about 'dispositions' such as fragility or brittleness. He argues that a variety of objects such as vases, parchments, spiders' webs, eco-systems and personalities all can be considered fragile, and yet these diverse kinds have no property or structure genuinely in common that is responsible for their fragility. Whereas 'dispositions' such as solubility, brittleness, or fragility are simply linguistic labels used across various kinds, 'dispositional properties' are genuine, or ontologically 'real' properties in the sense of contributing to the furniture of the world. Ellis goes on to argue that the manifestation of a behavioural disposition may have many and varied causes, but that a genuine dispositional property is intrinsic to an object and necessarily extant in all instances of that natural kind, to which the object belongs. All acids, for example, have the ability to supply protons in a chemical reaction, just as all electrons have a negative charge. (Ellis's natural kinds hierarchy will be described in more detail in Chapter 6) (Ellis, 2002, pp. 77-78).

The concept of 'intrinsic' or 'intrinsicness' has been the focus of considerable debate in philosophical literature over the last three decades. In 1983 David Lewis separated the notions of 'intrinsic' and 'internal' on the basis that some properties can be only partially intrinsic. These include, for example, being a brother, being in debt, or being located with respect to some place. According to Lewis, properties that are entirely intrinsic (e.g. shape, charge or internal structure), are internal (Lewis, 1983a,

\footnotetext{
${ }^{3}$ Examples of passive powers are the brittleness of a glass, or the solubility of a substance. If a particular possesses the power to either be acted upon, or to act, then the power (disposition) is a property of that particular. Thus, for Armstrong, properties 'bestow' powers (dispositions) on the particulars that have them (Armstrong, 1997, p. 69). Note that Armstrong does not differentiate between passive and active powers in his discussions on dispositions (p. 70).
} 
p. 197). This definition has been much discussed in papers on intrinsicness, with calls for a more precise delineation of properties and relations of objects that are corelational with other objects. The formulation of the term 'intrinsic' that I use is primarily aligned with that provided by Robert Francescotti's formal definition, given as follows:

$\mathrm{F}$ is an intrinsic property ${ }_{\mathrm{df}}$ necessarily, for any item $\mathrm{x}$, if $\mathrm{x}$ has $\mathrm{F}$, then there are internal properties $\mathrm{I}_{1}, \ldots, \mathrm{I}_{\mathrm{n}}$ had by $\mathrm{x}$, such that X's having $\mathrm{F}$ consists in x's having $\mathrm{I}_{1}, \ldots, \mathrm{I}_{\mathrm{n}}$. (Call a property that is not a d-relational feature of item $\mathrm{x}$ an internal property of x.) (Francescotti, 1999, p. 608).

George Molnar also gives an insightful definition of what it means to be intrinsic: 'intrinsic properties are those the having of which by an object in no way depends on what other objects exist' (p. 39). Stated by him more formally, ' $P$ is intrinsic to $x$ iff $x$ 's having $P$, and $x$ 's lacking $P$, are independent of the existence, and the nonexistence, of any contingent object wholly distinct from $x^{\prime}$ (p. 102). The definition of intrinsicness that I will use incorporates key concepts that are central to all of these definitions: an intrinsic property is one possessed by an object which is, itself, not $d$ relational to any other distinct object. That is to say, as Molnar explains, an intrinsic property is had by an object independently of the existence of any other object. The terms ' $d$-relational' (i.e. relational to any distinct object) and 'independent from', in the above, are similar conceptually to Langton and Lewis's use of the term 'unaccompanied' or 'lonely' to discuss objects not contingently co-existing with other (distinct) objects (1998, p. 343). ${ }^{4}$ Adopting a compatible view of relations, I will use the term 'intrinsic relations' to refer to relations between properties of unaccompanied objects, providing that these relations may never differ between duplicate pairs (i.e. pairs that have all of their internal properties the same).

\footnotetext{
${ }^{4}$ Further discussions on intrinsic properties and relations include that provided by David Lewis (1986c; 1999b). Brian Weatherson (2001) also provides a useful resource to the debate by reviewing sources of criticism of Langton and Lewis's account and by amending it to defend against these various criticisms (Handfield, 2009; Langton \& Lewis, 1998; Lewis, 1983a, 1986c, 1999a; Marshall \& Parsons, 2001; Sider, 2001; Yablo, 1999).
} 
Philosophers such as David Armstrong, Sydney Shoemaker, Ullin Place, Brian Ellis, Charlie Martin and John Heil are well-known contributors to the Metaphysics of Science debate concerning the nature of properties. The aim of this present Thesis is to explore the nature of properties in a comparative analysis of the writings of these pre-eminent philosophers. The primary focus is to enquire what kinds of properties are required at the fundamental level to provide a satisfactory explanation for the manifest world. Concerning fundamentality, the available options seem to include that: (i) dispositional and categorical properties are different kinds, both fundamental; (ii) dispositional and categorical properties are one and the same, and fundamental; (iii) only categorical properties are fundamental while dispositional properties, if they exist, are higher-order; and (iv) only dispositional properties are fundamental while categorical properties, if they exist, are higher-order.

I examine each of these positions and conclude that fundamentality is best explained without recourse to the categorical-dispositional distinction, which I believe does not exist at fundamental levels, but instead, only arises as a higher-order differentiation. I defend the claim that a coherent account of the manifest world can be given by starting with a pure-power base, which can be described in terms of powerful structure and is neither categorical in the sense of being independent of power, nor dispositional in the sense of being mere possibility or potentiality. There has been considerable criticism of pure-power theories, including via the Swinburne and neoSwinburne regress arguments, which together assert that pure power theories are unable to account for some kind of substance role, and for the ostensibly qualitative world. I respond to these criticisms, in part, by offering an account of the substance role fulfilled by fundamental power, and provide a plausible counterexample to the Swinburne regress, according to which the manifestly qualitative world might be explainable without recourse to fundamental categorical properties.

\subsection{Background Perspective}

In The ABC of Relativity, Bertrand Russell outlines two traditional conceptions of matter: first, Atomism - the view that at bottom matter consists of indivisible 'lumps' (1925, p. 206). A second view, forged by denying the existence of a vacuum, portrayed matter as an indivisible aether akin to a continuous field. Experimentally 
successful theories, arising with Michael Faraday and the mathematical research of James Clerk Maxwell on electromagnetic phenomena seemed to support this latter conjecture.

The history of physics in the early twentieth century points to a period of seesawing ambivalence concerning these two stances on the stuff of reality-applying to radiation also - being discrete or continuous. Russell noted in 1925, the very same year that Werner Heisenberg was completing his basic equations that became so important for Quantum Mechanics, that, 'relativity demands the abandonment of the old conception of "matter" which is infected by the metaphysics associated with "substance" and represents a point of view not really necessary in dealing with phenomena' (1925, p. 208). In 1927, Russell wrote that 'owing chiefly to two German physicists, Heisenberg and Schrödinger, the last vestiges of the old solid atom have melted away, and matter has become as ghostly as anything in a spiritualist séance' (Russell, 1970 [1927], p. 105). Matter, associated with Atomism, was thought to have given way to classical fields in which the distinctness of material points, heretofore assumed, fails.

Russell's idea of matter itself becoming as ethereal as a ghost at a séance', however, has not come to pass. The difference between his predictions and current eventualities may perhaps stem from the juncture of Maxwellian classical continuous fields and the quantised fields of Quantum Mechanics. In 1905, Albert Einstein's $5^{\text {th }}$ Paper, discussing the Photo-electric Effect, pointed out the contradictions that arise when applying the continuous spatial functions that operate with the Wave Theory of Light to the emission and transformation of light (Einstein, 1998 [1905], p. 178; Stachel, 1998b, p. 167). This paper challenged what had been accepted as the unlimited validity of Maxwell's theory; by suggesting that the effects of fields of influence occur in quanta or packets. As John Stachel notes, Einstein's solution was to propose that energy is not spread out continuously, but rather 'consists of a finite number of energy quanta that are localized at points in space, move without dividing, and can be absorbed or generated only as complete units' (1998b, p. 173). Einstein had spent a great deal of time considering the structure of both matter and radiation (Stachel, 1998a), and throughout his life he attempted to bring together a deterministic picture able to accommodate the quanta as well as continuous fields. In 1905, Einstein 
believed that physics and philosophy would proceed toward an explanation of the 'real' in terms of a continuous field theory of matter, recognising at the time of his writing that this had not been achieved. Later in life, Einstein noted that the thenpresent status of combining a view of material points with field theory had led to logical incompleteness:

Before Clerk Maxwell people conceived of physical reality - in so far as it is supposed to represent events in nature - as material points, whose changes consist exclusively of motions, which are subject to partial differential equations. After Maxwell they conceived physical reality as represented by continuous fields, not mechanically explicable, which are subject to partial differential equations. This change in the conception of reality is the most profound and fruitful one that has come to physics since Newton; but it has at the same time to be admitted that the program has by no means been completely carried out yet. The successful systems of physics which have been evolved since rather represent compromises between these two schemes, which for that very reason bear a provisional, logically incomplete character, although they may have achieved great advances in certain particulars (1934, p. 44).

Einstein noted that the Special and General Theories of Relativity, although based on ideas connected with field-theory, 'have so far been unable to avoid the independent introduction of material points and total differential equations' (1934, p. 45). This re-introduction of material points back into field theory can be read in terms of the quantisation of the classical field that had taken place in Quantum Mechanics, and has been influential in current metaphysical debate concerning spacetime structure. Brian Ellis, for example, considers that structure is the defining descriptor of the categorical. Since space and time are the 'pure forms of physical structure', categorical properties include spatiotemporal relations (2002, p. 174), and Ellis relies on these to underpin his natural-kinds hierarchy and its central tenet - that there is an ontologically-robust structure built into the universe (2001a, p. 174; 2001b, p. $2 ; 2002$, p. $68 ; 2005 a$, p. $382 ; 2008 a$ ). 
The claim that fundamental structure should be deemed categorical, however, is neither transparent nor theory independent. Alexander Bird (2005a; 2007, pp. 161168 ) and Stephen Mumford (2004, p. 188) suggest that this may be merely a matter of theoretical perspective. Quantum mechanical and earlier accounts treated spacetime as 'background', and this has contributed to the assumption that structure is categorical. As Bird notes, a spacetime geometry and metric that is background dependent leads to the idea of structure at fundamental levels being passive rather than active, and thence to the idea of categorical spacetime structure (2005a, p. 458). But, as discussed in more detail in Chapters 7 and 14 of this Thesis, this bias toward passivity is not necessarily justifiable. Background dependence presupposes an intrinsic metric such that spacetime corresponds to a fixed theoretical structure (Kribs \& Markopoulou, 2005, p. 4), interpreted by Ellis as categorical. However, this is contentious in view of recent scientific theories, including Loop Quantum Gravity (Smolin, 2000, pp. 106145; 2006) and the Bilson-Thompson Helon Model (Bilson-Thompson, 2005; BilsonThompson et al., 2009; Bilson-Thompson et al., 2007) that tend to treat spacetime dimensions either as powerful or as emergent from more fundamental, relationally derived foundations.

A second issue is whether the bias toward fundamental categoricity is tied to the intuitively attractive idea of fundamental particularity (i.e. haecceity or primitive thisness). As argued by Michael Redhead (1982) and Paul Teller (1982), the reality of particles in modern physics and more recently in metaphysics is highly debated, with many in favour of abandoning the traditional concept. Carlo Rovelli views a commitment to particle-hood as a long-standing inference formulated in spite of the fact that the particle-aspect of quantum 'entities' has never been detected and might be undetectable in principle (1997, p. 191). John Gribbin argues that the 'folk notion' of fundamental particles is basically a means to understand the mathematical laws describing fields of force, spacetime curvature and quantum uncertainty (1998, pp. 51-52).

The two issues outlined above concern whether fundamental categoricity exists in the form of either fundamental properties or fundamental particles. Although operating within a slightly different guise, the tension that Einstein highlighted, between explaining the structure of both matter and radiation, is still driving 
discussion in current Metaphysics of Science, especially in the theory of properties. It raises general questions about the nature of spacetime. In particular, what kinds of fundamental properties comprise the world, and how we might account for its ostensibly qualitative nature while recognising the existence of what appears to be fundamental power? Is there just one kind of fundamental property in the universe, or more? And if there is more - both categorical and dispositional, say - then what roles do they play and how do they interact?

If we could account for particularity and ostensibly categorical properties in terms of a more fundamental notion of pure power, this would shore up the stance that nothing fundamentally categorical exists - that only fundamentally powerful entities need to be postulated. However, arguments against this pure-power position include that of Richard Swinburne, whose regress argument (1980a, 1980b) was offered primarily in response to Sydney Shoemaker's Causal Theory of Properties ${ }^{5}$ (1980b; 1984a), and has been very influential in recent discussions. Shoemaker proposed that all genuine properties are sets of causal potentialities and are also to be identified with the clusters of conditional powers possessed by their bearers (1980b, p. 295; 1984a, pp. 217-221). Swinburne's response was to argue that a regress occurs for such a world in which there is nothing but powers (1980b, p. 317). If we recognise powers by their effects, and if these in turn are recognised in terms of the properties that are involved in those effects, then for a world in which these properties are pure powers, effects must be recognised by effects which must be recognised by effects, and so on. The alleged problem is that the 'properties' are never actually encountered, since each property is itself comprised of nothing more than effects. Swinburne claims that the regress can only be broken if there is something more to properties than powers (1980b, p. 317), a claim based upon the assumption that only categorical properties afford the direct detection of the contents of the manifest world, since effects themselves cannot be perceived directly.

Numerous 'neo-Swinburnian' arguments have followed. Armstrong presents the 'always packing, never travelling' argument - that the properties of pure power theories are never 'cashed' out in 'actuality' (1997, p. 80; 2000, pp. 13-14; 2004b, pp. 138-139). John Foster claims the need for space fillers (1982, pp. 66-72). Ellis claims

\footnotetext{
${ }^{5}$ Swinburne's regress will be discussed in more depth in Section 2 of this Thesis.
} 
that quiddities, comprising the fundamental structure of the universe, are required for the effects of power to be observable (2001b, 2002, 2005b, 2008a). Heil (2003a; 2006, p. 42) and Martin (1993, 1996a, 1996b, 1996c) conclude that something additional to pure power, namely the qualitative, is necessary for our sensing the objects of the world since they provide the 'what' or 'shell' of objects. Heil claims that, lacking this ingredient, anything resembling substantial nature dissolves and we are bereft of a coherent conception of material bodies (2003a, p. 107), since a nonqualitative world would supply insufficient resources to allow differentiation between empty and occupied space (pp. 76, 99-102).

In response, this Thesis argues that including fundamental categorical properties or particles in an ontology generates considerable difficulty in terms of justifying their presence and explaining their role. The body of literature under specific examination indicates two broad categories of thought-construable as monist and dualist — within which varying and competing stances concerning property fundamentality and type are positioned. Of the monist theories I have selected David Armstrong's Categoricalism and Sydney Shoemaker's Causal Theory of Properties as representing the respective stances that: i) only categorical properties are fundamental whereas dispositional properties are all higher-order; and ii) only dispositional properties are fundamental whereas categorical properties are higher-order. Description and analysis of these stances constitute Sections 1 and 2 of this Thesis. Of the dualist theories, I have selected the New Essentialist stance as put forward by Brian Ellis, and Ullin Place's Conceptualism, as representative of the view that categorical and dispositional properties coexist. While these two stances have in common that dispositional and categorical properties differ in kind, they diverge on issues of fundamentality and role. These two positions are described and discussed in Section 3 of this Thesis.

Section 3 also includes the Property Identity Theory, as put forward by Charlie Martin and John Heil. Martin's Dual-Aspect Theory depicted in his earlier work lends itself to being interpreted as a dualist theory of properties. However, his later Identity Theory presents as a monism, interpreted herein, as a clarification rather than a modification of the earlier Dual-Aspect Theory. The work of both Martin and Heil cannot easily be categorised as either monist or dualist, since it is both and neither: 
dispositional and qualitative (categorical) properties are irreducible to each other, yet they are the very same property.

Section 4 presents a discussion that, overlapping both Metaphysics of Science and Philosophy of Physics discipline areas, outlines and discusses field-theoretic ontologies. Chapter 12 provides an outline of the historical background of fieldtheoretic stances including those of Roger Boscovich (1922) and Immanuel Kant (1786/1909). These dynamicist approaches were influential in the development of Rom Harré and Edward H. Madden's ontology (Harré, 1970; Harré \& Madden, 1973; Harré \& Madden, 1975; Madden, 1972) — outlined and further discussed in Chapters 12 and 13-which in turn has influenced the Light-like Network Account put forward in Chapter 14 as a counterexample to the Swinburne regress.

The contribution of this Research Project is in terms of providing an in-depth, comparative analysis of contemporary ontological theories of properties. It also contributes to the topical discussion in Metaphysics concerning pure-power theory, while engaging with issues in fundamental physics, re-visiting and extending earlier field theories to arrive at a defense of pure-power theories in terms of accounting for the ostensibly qualitative world starting from a pure-power base.

\subsection{Delimitations}

This is a Thesis on ontology. In the 1970s and early 1980s, philosophical interest in questions of ontology_including discussion of fields and powers - was vigorous. Relevant philosophy included: Harré's and Madden's discussion of causal powers and the 'Great Field' (Cohen \& Madden, 1973; Harré, 1970; Harré \& Madden, 1973; Harré \& Madden, 1975, 1976; Madden, 1972; Madden \& Hare, 1971; Madden \& Sachs, 1972) and considerable literature generated by the ensuing debates (Frankel, 1976; Smith, 1982; Smith, 1984; Woller, 1982; Carr, 1978; Mackinnon, 1975; Cohen, 1973; Miller, 1972); Keith Campbell's survey of Spinoza, Leibniz and Boscovich and accompanying suggestion of physicalising spacetime (1976); Sydney Shoemaker's Causal Theory of Properties and what became a very influential response by Richard Swinburne and colleagues (Foster, 1982, 66-72; Robinson, 1982; Rosenberg, 1984; Shoemaker, 1980a, 1984b; Swinburne, 1980a, 1980b), Graham Nerlich's discussion 
of the shape of space (1976) and John Earman's theories concerning space and time (Earman, 1970; 1977).

However, the academic climate subsequently changed and, until quite recently, metaphysicians concentrated more on epistemological considerations, generating considerable discourse on ascriptional and conditional analysis of dispositions, and questions concerning issues of supervenience and reduction of properties. But there is at present, as noted by Martin and Heil (1999), an 'ontological turn' in progress. Evidence of this recent interest in ontology includes publication of books such as Armstrong, Martin and Place's Dispositions: A Debate (1996), George Molnar's Powers: A Study in Metaphysics (2003), Stephen Mumford's Dispositions (1998) and Alice Drewery's Metaphysics (2006). Other relevant material includes publications by Armstrong (1997; 2000; 2001, 2004a, 2005a), Shoemaker (1998), Place (1996d, 1999a; 1999b), Martin and Heil (Esfeld, 2006; Heil, 2003a; Heil, 2003b; Heil, 2005a, 2005b; Heil, 2005c; Martin, 1993, 1997), Ellis (1999, 2000, 2001b, 2002, 2005a, 2005b) and Alexander Bird (Bird, 2007).

Delimitations of this present Thesis include that it will concentrate primarily on questions of ontology rather than those debates that focus on property ascription and associated concerns. Writers such as Sungho Choi (2003, 2005, 2006), Michael Fara (2005), Lars Gundersen (2002), Marc Lange (2004), Isaac Levi (2003), Wolfgang Malzkorn (2000), Alexander Bird (1998) and Stephen Mumford (1998) have recently discussed these other issues at length. Discussions noted but put aside for the purpose of this Thesis include: first, issues relating to Martin's electro-fink thought-experiment $^{6}$ in which the circumstances for an object manifesting a disposition (e.g. touching a live wire) are the very same as would cause the object to lose (or gain) a disposition (e.g. upon touching the live wire, the wire goes dead) (1994). Second, Elizabeth Prior, Robert Pargetter and Frank Jackson's swamping objection - $\alpha$ might be fragile but did not break in appropriate circumstances because some other disposition competed with and swamped the effect (1982). Third, a similar discussion by Bird and others with respect to masking - a poison might have the ability to kill, but an antidote is given (1998). Fourth, mimicking - the manifestation

\footnotetext{
${ }^{6}$ According to Michael Fara, Martin's objections to simple conditional analysis were first raised in 1957, although not published until 1994 (Fara, 2006).
} 
that is identical to that expected of a certain disposition, but comes about in a way independent of the disposition, e.g. the destruction of a dropped chalice due to an angel's act rather than by virtue of being dropped (Johnston, 1992; Smith, 1977). Fifth, the non-existence of the categorical-dispositional distinction in terms of entailment (Bird, 2003; Mellor, 1974; Prior, 1985; Prior et al., 1982) and questions concerning supervenience and reduction of properties (Kim, 1990; Mumford, 1994).

The second delimitation is that this Thesis makes use of representative samples to examine key positions concerning the nature of properties. This methodology affords certain depth and breadth of coverage across the discipline. However, an examination of every possible position is not within the scope of this project. I have, therefore, restricted the range of analysis to include only those positions that hold in agreement with a theory of strong causation. Accordingly, this Thesis does not address those theories, such as those postulated by David Lewis (1983b, 1986b; 1986d; 1992; 1993, 1999a; 2000; 2001), that defend a neo-Humean regularity theory of causation, although Humean event ontology is discussed briefly in Chapter 12 with respect to the work of Rom Harré. It may well be that a Humean view favours a theory of fundamental categorical properties, since it is under no burden to account for the necessity required of strong causal theories. Putting aside whether or not such a theory is satisfactory in terms of explanatory power concerning causality, an examination of its internal consistency with respect to a fundamental categoricity will be an interesting future project. 


\section{SECTION 1:}

\section{CATEgORICALISM}

Section 1 outlines and discusses Categoricalism, a property-monist view put forward by David Armstrong. Categorical Monism restricts the domain of 'real' properties to the purely categorical. Objects participate causally in the world by means of dispositional properties that are supervenient upon the categorical microstructure of their object-bearers. This supervenience, according to Categoricalism, depends upon prevailing, contingent laws of nature. Armstrong also defends a view of strong causality according to which connections exist between instances of cause and effect that, being instances of nomic types, amount to more than Humean regularities. Such a view faces the challenge of explaining where the necessity required of strong causation arises in a world devoid of irreducible dispositional properties. 


\section{ChAPTER 2 \\ DAVID ARMSTRONG: \\ CATEGORICALISM - OUTLINE}

In this chapter, I outline the evolution of Categoricalism, beginning with Armstrong's early reductionist approach to dispositions through to the later Supervenient Thesis. I also briefly address the 2004 tentative revision concerning instantiation and the status of laws of nature, and plausible reasons for Armstrong's subsequent re-thinking of that revision.

\subsection{The Early Reductionist Thesis}

Armstrong's account of dispositions begins with a paper entitled Nature of Mind (1966). ${ }^{7}$ Dispositions were further addressed in his book, A Materialist Theory of the Mind (1968). In these publications, Armstrong responds to Gilbert Ryle's Phenomenalism according to which a disposition is merely a predictive description of behaviour. Two conceptual differences between the views of Ryle and Armstrong stand out: first, for Ryle dispositions are constituted by behaviour, whereas for Armstrong dispositions underlie behaviour. Second, for Ryle there exists, at best, a contingently existing connection between dispositions and the categorical bases of objects (or persons) possessing them. For Armstrong, however, this connection is deemed necessary.

Ryle believes that the possession of a dispositional property is merely the fact that a thing possesses an ability or liability for change under certain circumstances. $\mathrm{He}$ writes, 'To possess a dispositional property is not to be in a particular state, or to undergo a particular change; it is to be bound or liable to be in a particular state, or to undergo a particular change, when a particular condition is realised' (Ryle, 1949, p. 43). He describes, for example, the disposition of solubility: 'To say that this lump of sugar is soluble is to say that it would dissolve, if submerged anywhere, at any time and in any parcel of water (Ryle, 1949, p. 123). An object's brittleness, for example, would be merely the fact that things of that type break easily if subjected to certain

\footnotetext{
${ }^{7}$ This was re-published in Armstrong's collection, The Nature of Mind and Other Essays (1980).
} 
conditions. For early Armstrong, however, dispositions are not merely abilities or capacities, but underlie the abilities or capacities (1968, pp. 86-88; 1980, pp. 9-10). To bequeath them ontological-robustness, dispositions are identified with the state of the categorical base of their bearer (1968, pp. 88-89; 1980, p. 9; 1984b, p. 22), and as such, are nothing over and above the categorical microstructure (1968).

In contrast to Ryle's contingently existing connection, Armstrong's identity between the disposition and the microstructure amounts to a necessarily existing connection. The claim to theoretical supremacy by Armstrong lies in the fact of Ryle's categorical base failing to warrant ascription of dispositions that have not (yet) manifested (1968, pp. 86-87). For a realist about dispositions, such as Armstrong, an unmanifested disposition can supposedly be explained in virtue of its being a possible state of an ontologically-robust categorical base. Given its status as physically existent, this can be treated as the truthmaker for the present existence of the unmanifested disposition (Armstrong, 1968, pp. 86-87). The metaphysical reduction of dispositions advocated in early Armstrong is type-type identity; types of disposition (e.g. brittleness, belief) are ultimately identified with types of microstructure (e.g. glass, certain mental state) (1983a, pp. 57-58). Even if some token categorical base is not presently in some token state, similarity of microstructural types justifies inference of similarity of properties. Thus, one can justifiably infer an unmanifested disposition to a certain microstructural base so long as somewhere at sometime such types of bases have manifested the relevant types of dispositions in relevant circumstances.

As the type-type identity in question describes a necessary connection between dispositions and categorical microstructure in general, clarification is required concerning the contingent identification that Armstrong posits between mental and cerebral states in his Central-State Materialism. In this Theory, a first step reduces mental dispositions (e.g. beliefs) to their 'non-dispositional' (but mental) categorical bases (e.g. 'inner' mental states). This reduction represents a necessary connection between the inner-state and the disposition. However, in a second reductive step, this inner mental state is only contingently (or 'scientifically') identified with its physical categorical base (i.e. brain-state) (1968, p. 91). Armstrong claims 'belief' to be an 'inner state' or disposition $(1968$, p. 88). In this case we differentiate physical from 
mental dispositions as in Figure 2.1: physical dispositions, like brittleness, are necessarily identified with the physical substructure of their bearer; mental dispositions are necessarily identified with inner mental states, then contingently and indirectly identified with their ultimate physical base. Whether physical or mental, the disposition is metaphysically reduced to its basis - physical dispositions to a physical basis and mental dispositions to a mental basis, the latter being further contingently identified with its physical basis.

Figure 2.1 Physical versus Mental Dispositions

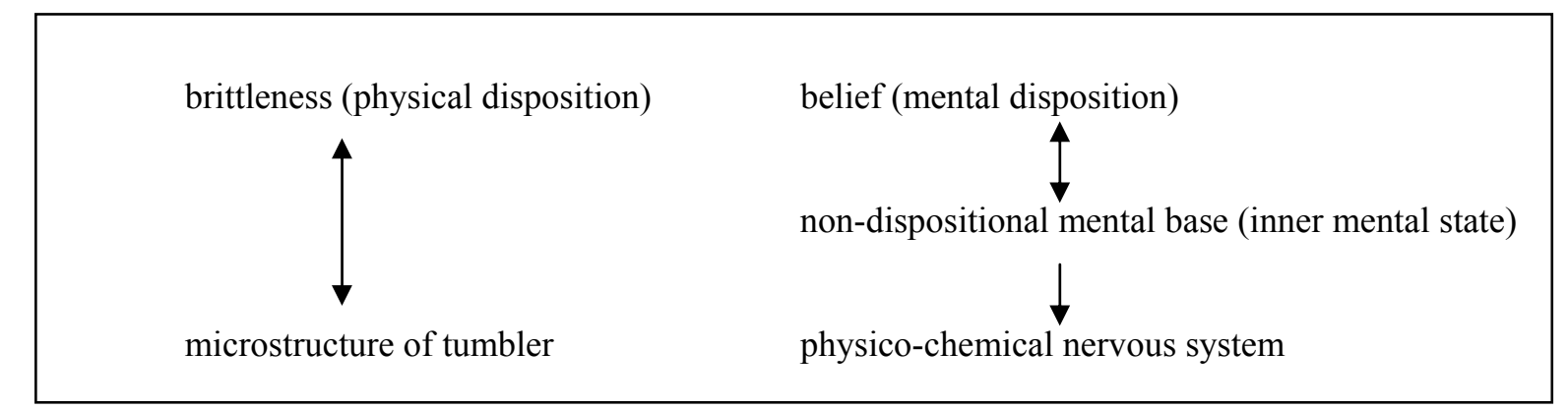

Key: double-headed arrow: metaphysical reduction; single headed arrow: contingent reduction

\subsection{From Metaphysical Reduction to a Supervenient Thesis of Dispositions}

The late 1970s and early 1980s saw the migration of Armstrong's metaphysical reduction of dispositions to the Contingent Identity Thesis. Token-token (or more accurately, sub-type-sub-type (Armstrong, 1997, p. 73)) identity replaced the earlier type-type identity, and the laws of nature became central to the discussion. This section outlines the process of evolution from the earlier Reductionist Thesis to the Supervenient Thesis.

Multiple-realisability generated problems for the Type-type Identity Thesis. Armstrong notes the Functionalist observation that different sorts of things perform the same functions (1983a, pp. 57-58). An elastic band, for example, possesses the disposition of elasticity (1984a, p. 139). According to Type-type Identity Theory, the elasticity is identical with the microstructure. But if we understand 'elasticity' to be the disposition of elastic things to change their shape under strain, and then return to their original shape once the strain is removed, microstructures of many objects (rubber balls, springs, viscoelastic polymers) correspond to 'elasticity'. Moreover, an 
elastic band possesses various dispositions, including colour, thickness and so on. Multiple-realisability of dispositions thereby ruled out types of dispositions being identified with types of microstructures.

Transitivity turned out to be a second, closely related problem for type-type identity. As described by Elizabeth Prior and colleagues (1982, p. 253), if a particular disposition is grounded by base $(\alpha)$ in one object and by a base $(\beta)$ in a second object, then, since each disposition is identified with its base, by transitivity, $\alpha=\beta$. Let us say that $\alpha$ has a biological microstructure of some sort that gives it fragility; and $\beta$ has a crystalline microstructure that makes it fragile. By transitivity, the biological microstructure of $\alpha$ somehow is the crystalline microstructure of $\beta$ (Prior et al., 1982, p. 253). Armstrong's response to these concerns was to change from type-type to token-token (or at least sub-type-sub-type) identity (1997, p. 73).

Although solving the problems associated with multiple-realisability and transitivity, this theory still involved metaphysical reduction, as indicated by Armstrong's continuing to identify an object's properties (microstructural or categorical) $^{8}$ with its causal powers (dispositional properties). For example, his 1972 paper establishes an identity-condition for properties such that, 'for each distinct property a thing has, the thing has distinct causal powers' (1972, p. 176). This identity is upheld as late as 1978 and beyond, with Armstrong linking the 'sameness' or 'difference' between universals with the sameness or difference between powers, respectively (1978a, p. 50). Stephen Mumford emphasises the naturalness of identifying properties with powers; if we believe two things differ in their abilities, potentialities or powers, we tend to explain that difference by pointing to some discrepancy in their material composition (1998, p. 29). For example, given two tumblers identical in every microstructural way, it would seem absurd that one is brittle and the other is not.

The change to token-token metaphysical reduction was not completely successful, however, for it still succumbed to the following possible-worlds objection: if an object's disposition is identical to its microstructure, then, given the identity derives from metaphysical reduction and is therefore necessary, in all possible worlds

\footnotetext{
${ }^{8}$ All true properties are considered by Armstrong to be categorical.
} 
in which that object exists the disposition must exist also. For example, if $a$ is a brittle object, then in all possible worlds in which $a$ exists, it is brittle. But this suggests that: i) the microstructure of an object in one world is functionally equivalent to the same microstructure in all possible worlds; and therefore, ii) the laws of nature of any possible world in which some microstructure exists are the same in all possible worlds in which that microstructure exists, i.e. are necessary laws.

Like many theorists, however, Armstrong considered the laws of nature to be contingent (1997, p. 72), which meant abandoning his token-token metaphysical reduction in favour of a token-contingent identity between dispositions and their microstructural bases. Thus Armstrong replaced the microstructure-causal power identification with a microstructure-causal role identification. The predicate 'elastic' picks out some microstructural property of an object, but does so by targeting the role that that property plays in allowing that object to stretch under strain. In this way, Armstrong allows for different structures of things to play the same role (1984a, p. 140). Thus a causal property of an object is picked out via its causal role in bringing about some manifestation, rather than via its intrinsic nature (1996b, p. 39).

This solution involves the laws of nature because, by proposing that although dispositions are nothing over and above the microstructure of their bearers, a particular microstructure together with the laws of nature determines the specifics of the causal role. The laws of nature 'ensure' the contingent identity between microstructure and disposition, as the following example, given by Armstrong, shows: let disposition $\mathrm{D}$ be a categorical (non-relational) property of the microstructure of object $a$; and let $\mathrm{E}$ be the effect of manifestation of D. Suppose $a$ has the brittleness, $\mathrm{D}$, to break in suitable circumstances. Then, as Armstrong says, since dispositions are to be identified with their microstructural base (albeit contingently):

D will have to be some categorical property of $a$, connected with the breaking, E, only by laws of nature (which are contingent)... The position may be stated in terms of supervenience. If $\mathrm{D}$ is taken as a categorical property, then $a$ 's brittleness is supervenient on the fact that $a$ has $\mathrm{D}$, together with the laws of nature that ensure that, in suitable circumstances, an $a$ that has D breaks (1989, pp. 117-118). 
This position moves away from token metaphysical reduction to a supervenient, Contingent Identity Theory of dispositions; strong identification of dispositions with their microstructures is replaced by contingent identification, supplied by the arbitrating influence of the laws of nature. Nonetheless, John Bigelow and Robert Pargetter argue that this change does not allow a disposition the status of being anything over and above the microstructure of its bearer. Rather, it merely represents the fact that a given property can be described in two different ways (1999, p. 625). If something is 'soluble', it does not have a property of 'solubility' plus a certain chemical structure. Rather, we just use two different terminologies to describe the fact that the substance is able to dissolve in certain circumstances. Armstrong's token-contingent view still permits identifying dispositions with their categorical base, provided we understand this as contingent (Armstrong, 1989, p. 118). The same substance may not have the ability to dissolve in those same circumstances in another possible world where the laws of nature differ. Armstrong thereby retains an identity between token dispositions and token microstructures without being compelled to defend necessary laws.

In this Contingent Identity Theory, although the causal role of tokendispositions is contingent upon the laws of nature, the laws are not causal factors in the manifestation of these dispositions. Armstrong writes, 'Notice that the laws are not causal factors. The causal factors, real and feigned, bring about the manifestation, in accordance with the relevant laws, and not with the addition of the laws' (1996b, p. 41). As he further notes:

[B] ut it may still be defensible to identify the brittleness with the bonding. Consider that the first-order state plus the laws will, in the given conditions, be sufficient to entail (or give a certain objective change) that a suitable striking will shatter the object...Given this, cannot one say that, relative to the laws, the first-order state is the disposition, and then leave this relativity in the semantic background? (1997, p. 73). 
The difference between laws playing a causal role and being causal factors is significant. Causal factors are the microstructural categorical properties. The laws, being contingent, cannot be these properties. Armstrong contends instead that the various properties of the microstructure are relata, linked in certain ways according to laws. Thus, although the laws are contingent and therefore extrinsic to the microstructural properties, they are partial truthmakers for the existence of dispositions, the complete truthmaker being the composition plus the relevant laws of nature (1997, pp. 72-73). The term 'truthmaker' was introduced to current metaphysical use by Charles Martin (following Gustav Bergmann) in describing truthmakers as the 'ontological ground' by which statements are true (Armstrong, 1997, p. 13). If the laws and microstructure are together the truthmaker for a dispositional ascription; and if the relation between truthmaker and truth is internal; then the laws cannot stand to the disposition extrinsically, although they stand to the microstructure extrinsically. Armstrong captures this idea in claiming supervenience of dispositions to be both ontologically reductive, yet semantically non-reductive:

A supervenience thesis is often thought of as a reductionist thesis, or perhaps as a superior substitute for reduction. It is superior because it promises to cut down on entities without necessarily demanding that statements about the supervenient entities be translated into statements about the entities on which the supervenient supervenes. Perhaps it might be said to be ontologically reductionist, without being semantically reductionist (1989, p. 104).

The supervenience incorporates the 'ontological free lunch', according to which, whatever supervenes is nothing ontologically over and above its subvening base. In the case of dispositions, while we refer to the term 'disposition' or ascribe such to objects, these predicate terms correspond to the ontologically-robust categorical microstructural properties and laws that ground them. The gist of the ontological free lunch is that, as Armstrong notes, 'You get the supervenient for free, but you do not really get an extra entity' (1997, p. 13). (Armstrong upholds this doctrine with a few exceptions (e.g. for totality states of affairs)). Since dispositions are nothing over and above their categorical basis together with the laws determining 
what the objects-bearers of categorical properties can do, then accounting for power in the world falls heavily onto the laws. This is a topic further addressed later in this chapter, and also in Chapter 3.

In summary, responding to problems of multiple realisability and transitivity, Armstrong moves from type-type metaphysical reduction to token-token reduction. To accommodate contingent laws of nature into this ontology, his view then evolves into a token-based Contingent Identity Theory of dispositions, whereby the identity of token disposition and token microstructure occurs with respect to contingent laws of nature as partial truthmaker for dispositional ascriptions. The role of laws as partial truthmakers for dispositions and their manifestation allows dispositions to be accommodated within the possible-worlds discourse, while still (contingently) identified with the microstructure of their bearers.

\subsection{States of Affairs and their Constituents}

The previous section focuses specifically on the evolution of Armstrong's Contingent Identity Thesis. This section provides a broader picture of his Combinatorial Theory. Thus far, I have used terms such as 'universal' and 'property' fairly loosely, but here I will describe more precisely what Armstrong means by these and by 'states of affairs'.

\subsubsection{States of Affairs}

Armstrong's Combinatorialism is a theory of possibility, starting from a world of states of affairs comprising individuals and universals. An atomic (first-order) state of affairs exists where a particular has a property, or where a relation holds between two or more particulars (1997, p. 20). A particular having a property is represented as $\mathrm{F} a$, where $a$ is a particular with the property F. An atomic (first-order) state of affairs in the case of a relation between two particulars is consistently represented by Rab, 'a state of affairs involving first-order particulars falling under a first-order universal' (Armstrong, 1983b, p. 88). Universals, general, are considered to be properties that are able to be multiply located, and are wholly present in each of its instances.

Just as complex or structured properties and individuals are composed of parts, so molecular and higher-order states of affairs are composed of atomic states of 
affairs. Armstrong upholds the Thesis of Independence: two states of affairs are independent if and only if no conjunction of them entails either the existence or the non-existence of any wholly distinct state of affairs (1997, p. 139). Atomic states of affairs, for example, are independent (1997, p. 147).

Molecular states of affairs should be differentiated from higher-order states of affairs (1997, p. 122). Molecular states of affairs are comprised of further states of affairs standing in a part-whole relation to each other. In this case, or in the case of overlapping states of affairs, Armstrong describes that independence does not hold, since they are compositionally mereological. He describes, for example, an overlapping state of affairs such as his townhouse having walls in common with its neighbours. He argues that should its neighbouring townhouses disappear, he would be missing two walls, which shows that his townhouse is not independent from the neighbouring ones. Instead, this is a case of partial identity, such as is the case in molecular and/or overlapping states of affairs; and is not compatible with independence (1997, p. 18). A molecular state of affairs is supevenient upon, and is nothing over and above, its constituent parts. In comparison, higher-order states of affairs are non-supervenient (Armstrong, 1997, p. 118), demonstrated by the possible existence of two quite independent states of affairs comprised of the very same constituents (Armstrong, 1997, pp. 118, 196). At least two kinds of higher-order states of affairs are included in Armstrong's ontology: first, totality states are described as collections of first-order states of affairs; and second, laws of nature that contingently link universals (i.e. state of affairs types) (Armstrong, 1997, p. 2). Whereas $\mathrm{F} a^{9}$ is a first-order state of affairs, a state of affairs having $\mathrm{F} a$ and $\mathrm{F} b$ as constituents would be a second-order state of affairs, represented by $\mathrm{F} a$-R-F $b$. Unlike molecular states of affairs, totality states of affairs are non-supervenient, and thus do represent an existent over and above its constituents. In the case of totality states of affairs, only one-way independence holds; that is, given the existence of the higher-order state of affairs, its constituents must exist, although the existence of the very same constituents would not necessarily entail a higher-order state of affairs. Moreover, the characteristics of

\footnotetext{
${ }^{9}$ Armstrong uses the regular capital letters of the Roman alphabet to indicate properties; and small, italicised letters of the Roman alphabet to indicate particulars possessing those properties.
} 
this higher-order state of affairs will differ from those of its constituents (Armstrong, 1997, p. 118).

Armstrong introduces the notion of universals as 'state of affairs types' into his ontology. States of affairs are not universals, but particulars (non-repeatables). Armstrong calls this 'the victory of particularity' (1978b, chapter 11, section III; 1989 , p. 52). Hence, Fa and Rab are particulars. The expression 'state of affairs type' does not suggest that universals are themselves states of affairs, but emphasises that all states of affairs instantiating a given universal possess that universal in common. The $\mathrm{F}$ instantiated by $\mathrm{F} a$ is a first-order universal. $\mathrm{But} \mathrm{F} a, \mathrm{~F} b$, and $\mathrm{F} c$ are all states of affairs with the property $\mathrm{F}$ in common. In this sense $\mathrm{F}$ is a type of state of affairs, but since the individuals $a, b$ and $c$ have only the barest of haecceity, we abstractly remove the particularity of each and regard them as instances of a type of property (1989, p. 44). In Armstrong's scheme, $\mathrm{F} a$ is a first-order state of affairs, but $\mathrm{F} a$ is also a state of affairs of type F. This type-F, argues Armstrong, is itself a universal, because it is a type of state of affairs and hence repeatable in its instances. So we have two universals: F (first-order) and type-F (higher-order). Armstrong (1983b, chapter 6 , section 4; 1997, sections 3.5, 15.1) provides detailed discussion of state of affair types. These play an important role in his theory of causality. Observed regularities of 'this causing that' point to some underlying causal connection between state of affairs types. However, because uninstantiated universals do not exist, these types exist only in their instances, albeit the instances occur according to patterns among the types.

\subsubsection{Particulars, Properties and Relations}

Armstrong's states of affairs take as their constituents particulars, properties and relations. It is a combinatorial theory in the sense that it begins with simple individuals that constitute complex or structured objects. 'Simple' individuals are not constituted by proper parts (1989, p. 38), but neither do they exist bare of properties and relations to other individuals. Armstrong notes that we cannot say dogmatically that simple properties actually exist, since reality might be structured 'all the way down' (1978a, p. 68; 1989, p. 113; 1997, p. 33), but they are a conceptual beginning for his combinatorial ontology. Examples of simple properties might be point-instants, mass and charge (envisaged non-relationally). Simple relations include spatiotemporal 
and causal relations. These simple properties and relations are viewed by Armstrong as universals (Armstrong, 1989, pp. 38-39).

Armstrong's theory admits higher-order properties and relations - those with constitutive parts - such as conjunctive and structural universals. If $a$ instantiates both $\mathrm{F}$ and $\mathrm{G}$, where $\mathrm{F}$ and $\mathrm{G}$ are wholly distinct universals, then $a$ instantiates the conjunctive universal F\&G. Also, if $a$ is $\mathrm{F}$ and $b$ is $\mathrm{G}$, with $a$ wholly distinct from $b$, and if $a$ has relation $\mathrm{R}$ to $b$, then $[a+b]$ instantiates the structural universal made up of an F-part having R to a wholly distinct G-part (1989, p. 113). However, these conjunctive and structural universals supervene upon simpler constituents and are nothing metaphysically extant over and above simple universals.

Universals are repeatable such that they can be instantiated by an indefinite number of individuals. They are the same property and/or relation across different instantiations (1989, p. 39). Armstrong outlines three important differences between universals and individuals: i) Properties and relations are predicated $o f$ individuals but not vice versa. Thus, a property is of an individual; but an individual is not of a property; ii) when an individual possesses a property, it is said to instantiate that property. Likewise for relations. However, for a property or relation to be a genuine universal, it must be identical in all its instantiations, suggesting that a Principle of Instantial Invariance applies (1989, p. 40): for a relation to be a genuine universal, it cannot take different numbers of terms in different instantiations. For example, it cannot relate $a$ and $b$ in one instantiation, but $c, d$, and $e$ in another. A simple nonrelational property is always monadic - wholly present in its individual instantiation; and a given simple relation is always dyadic, triadic or so forth, such that the number of terms of a universal is fixed. Conversely, providing that an individual has at least its individuating property, the number of properties and relations that it instantiates is open; leading to iii) all simple individuals are identical apart from their individuation. In Armstrong's words, 'In abstraction from their properties and relations they are barely numerically different' (1989, p. 44).

Armstrong does not allow the existence of bare particulars, since particulars need at least 'particularisation'-the property of individuation, or numerical individuality - in order to exist (1989, p. 32; 1997, p. 109). The question of whether he allows thin particulars is more controversial. A thin particular is one that is devoid 
of all properties other than its 'particularising property'. His position on thin particularity seems to have changed in line with the adoption of a deflationary account of haecceity in 1997. I briefly outline this evolution in the passages that follow.

In 1978, Armstrong held spatiotemporal position to be a unique property; one that was not to be viewed as a universal in the manner of other properties such as size and shape. Rather, it was considered to be the property that particularises a particular. Discussing the proposition that, 'sameness of total position and sameness of nature necessitates sameness of particulars' (given as proposition 5), Armstrong argues that:

I believe that we are now in a position to substantiate, though not actually to prove, the suggestion that spatio-temporal or total position does, as a matter of fact, constitute the particularity of particulars (at least for all those particulars which are spatio-temporal in nature, which in my view are all the particulars there are). Proposition (5) appears to entail that each spatio-temporal particular, concrete or abstract, is some sort of unity of its properties (its properties include its spatio-temporal properties) and its total position. Between a nature thus conceived, and its total position, we appear to be able to make only Scotus' "formal distinction". It is not easy to identify the total position with any further property, relational or non-relational, of the particular. So spatio-temporal or total position appears to constitute the particularity of spatio-temporal particulars. By adding this particularity to a thing's nature, or properties, we gain a particular: the 'victory of particularity'...If spatio-temporal position had been a property, adding it would simply have augmented the thing's nature, and nature by itself is repeatable, i.e. it does not by itself constitute a particular. That fact that different ("abstract") particulars might all have the same position merely shows that the particularity of a particular does not completely determine that particular. For such a completely determination, we also require its nature. This is no more than the doctrine of the this-such (Armstrong, 1978b, p. 124). 
Duns Scotus' formal distinction allowed for a merely formal, yet real, distinction between the thisness of a particular and its 'forms' or properties (Armstrong, 1978b, p. 109). (Duns Scotus' formal distinction is further described in: Boler, 1963, pp. 5355; Grajewski, 1944, pp. 97-101; Scotus, 1994, d. 3, part 1, q. 6, p. 107; 1997, I, d. 33, q.2, pp. 329-334). This formulation was borrowed from Aristotle's notion of 'thissomething', sometimes translated as 'this-such'. Aristotle had written, 'we must also ask whether principles are general or, as we say, "individuals". If they are general, they are not primary beings; for what many beings have in common cannot itself be a this-something, but is a "what"; whereas a primary being is a "this"'(Aristotle, 1952, Book Beta (III), 6, 1003a, p. 60). For early Armstrong, particulars are not only 'such' - they also have a 'thisness' to them that makes them distinguishable from properties (Armstrong, 1978b, pp. 109, 116). Since Armstrong, at this stage, viewed the nature of a particular solely in terms of its qualitative properties such as size or shape, he needed to provide for the individuation of a particular apart from such properties. He opted for spatiotemporal position to do so. Spatiotemporal position was thought by Armstrong to be no ordinary universal, seen as providing numerical differentiation while not adding to the qualitative nature of the particular. The 'thissuch' tie between this particularising property and a particular afforded the existence and numerical independence of the particular, which as Armstrong notes, is the 'Victory of Particularity' Principle in play. He further clarifies this description of the particularising property:

We might call the truth involved the Principle of Particularisation. It is the truth that, for each particular, there exists at least one monadic universal which makes that particular just one, and not more than one, instance of a certain sort. Such a universal will be a "particularizing" universal, making that particular one of a kind. Without such a universal, the particular is not restricted to certain definite bounds, it is not "signed to a certain quantity", we do not have a "substance", we do not have a particular... What will the particularising universals be? I can see nothing which is always available except the spatiotemporal pattern possessed by the total or spatio-temporal position of the particular involved (Armstrong, 1978a, p. 64). 
By 1989, the particularising property was considered to be a spacetime point, which still built in the notion of spacetime position (1989, p. 32) and acted as a place-holder for the banished notion of fundamental haecceity:

But could an individual be propertyless? Can it exist, but not in any particular way? I do not think it can. An individual, to be an individual, must be one thing. But to be one must it not 'fall under a concept', as Frege would put it, that is to say, have some unit-making property? Without that, it is not even an individual. ...I shall argue in Chapter 4 that there is (a) sense in which individuals are all the same. In abstraction from their properties and relations they are barely numerically different. (This is the rejection of the doctrine of haecceity) (Armstrong, 1989, pp., 43-44).

Dismissing the option of adopting a Haecceitist view, and differentiating between a strong anti-Haecceitist position (i.e. a bundle view of universals $(1989$, p. 59)) and a weak anti-Haecceitist position, Armstrong defends the latter, writing, 'I suggest, therefore, that the Naturalist-Combinatorialist [such as Armstrong] should move to the weak anti-Haecceitist position' (1989, p. 60). Thus, it can be argued that the earlier adoption of the particularising property was tied to the rejection by Armstrong of the doctrine of haecceity, which had resulted in spacetime location being called upon to supply individuation of particulars.

By 1997, Armstrong had adopted a 'moderate' or 'deflationary' form of haecceity, writing that he thinks his earlier anti-Haecceitism was too extreme (1997, p. 108, 168). Nonetheless, while still maintaining that, 'the nature of a thing is given exclusively by its properties', he asserts that, '[w] hen we have said that different particulars are numerically different, then we have said all that can be said about the nature of particularity' (1997, p. 109). This appears to be an unsatisfactory move, since he is left with no explanation for individuality other than it being fundamental and unanalysable. This new position leads Armstrong to reject the role of haecceity being had by anything 'property-like', although it still requires his individuals to be 'numerically different'. This change was expedited partly in order to accommodate 
the possibility that spatio-temporal position may not be available to play the individuating role, as he had once suggested:

Second, it is not at all obvious that two particulars could not exist at the same place and time...Perhaps the fields of physics are real things, as contemporary physics tends to assume, and can interpenetrate. Perhaps there can even be two particulars with exactly the same properties at the same place and time. If so, difference of spatiotemporal position is not necessary for numerical difference. Third, if there are or can be particulars that remain numerically and strictly identical over time, then difference of spatiotemporal position is not even sufficient for numerical difference (Armstrong, 1997, p. 109).

As Armstrong notes, the upshot is that from 1997 onwards 'the particularity of particulars is taken as fundamental and unanalysable' by him, and the once understandable difference between bare and thin particulars ceases to be considered (1997).

Clearly, thin particulars play an important role for Armstrong, who uses them to argue against a bundle theory of properties (1997, pp. 95-99, 123-126). Armstrong's states of affairs require a bearer or instantiator in addition to the properties and relations instantiated. Moreover, if properties are 'ways' in which particulars are, this is more reason for thin particulars being theoretically indispensable to his ontology (pp. 122-124). The thin particular, then, forms the skeleton of the thick particular-the fully-fleshed entity.

On one hand, some might argue that Armstrong's support of direct perception of particularity, over and above any experience of properties and relations (pp. 122123), leads to the existence of thin particulars in his ontology. On the other hand, it seems doubtful that Armstrong allows his thin particulars to be 'unclothed', and if clothed they constitute 'thick' particulars. From this it appears that Armstrong's thin particulars are nothing more than abstract entities, or pre-forms of thick particulars. Discussion of thin particulars are taken up in more detail in Chapter 3.

In contrast to Armstrong's Universalism, Nominalism denies repeatability of properties. Issues pertaining to differences between Universalism and Nominalism are 
outside the scope of this Thesis, but a short note here simply highlights the existence of alternative stances to that of Armstrong. Both Universalism and Nominalism present certain difficulties. Central to the controversy is how to explain different objects seeming to possess the same properties. What, for example, is meant by saying that a glass tumbler and a china plate both possess the very same property of brittleness?

A Nominalist stance may require accepting certain brute facts concerning exact resemblance of tropes. However, as Bertrand Russell notes, the relation of resemblance itself appears to be a universal: given something white, it must resemble a particular already taken to be white with respect to colour, and this resemblance holds between all pairs of white things, which is just the repeatability proposed of universals (1911). Armstrong argues that theories which reject universals must embrace some kind of fundamental exact resemblance to explain the similarity of things (1997, pp. 22-24).

By comparison, the repeatability of Armstrong's universals explains why things may seem to be of the same type, and act in similar ways. If particulars instantiate the same properties and relations, then the similarity of particulars is understandable. David Lewis notes a major advantage of Armstrong's position is that universals unify reality by accounting for how things have properties in common (1983b, pp. 344-345). However, as discussed in more detail in Chapter 3, this repeatability, and the fact that universals exist only in their instantiation rather than in some Platonic Ideal realm, are put forward as facts partially derived from, or at least tied to, the notion of universals as able to be multiply located. This multiple location appears to be fundamental and unanalysable. Armstrong strongly links multiple location and repeatability when he writes, 'I think the repeatability of universals is a further mark of differentiation. There is no modal limit to their being instantiated at indefinitely many places and time (Armstrong, 2004a, p. 147). Although repeatability may play an important explanatory role, it is itself left unexplained. Moreover, the nature of instantiation of universals, and the 'non-relational' tie that Armstrong introduces to explain how properties are 'had' by individuals, is also far from transparent. These issues will be further discussed in Chapter 3. 
In summary, properties and relations are universals in Armstrong's ontology, with strictly identical repeatables exemplified in different instantiations. Contra transcendent Platonic universals (Ideal Forms), Armstrongian universals do not exist unless and until instantiated by a particular. An instantiating particular together with at least one property constitutes an atomic 'fact' or 'state of affairs', providing this is not composed of further states of affairs (1997, pp. 19-20). Any state of affairs is a particular, not a universal, although the property it instantiates is a first-order universal. State of affairs types are higher-order universals.

\subsection{Laws of Nature}

Some define power in terms of the ability of objects to affect or be affected in virtue of their dispositions. However, for a Categoricalist, dispositions (including powers) are grounded in the categorical microstructure, and objects engage causally only by virtue of the power that they derive from their dispositional properties, contingent upon the laws of nature (1997, pp. 82, 205). These laws are relations between universals, according to a concept independently proposed by Armstrong (1978a), Fred Dretske (1977) and Michael Tooley (1977) in the 1970s (Armstrong, 1983b, p. 85).

For Armstrong all 'true' properties and relations, being categorical, have natures that are self-contained in the sense of being 'distinct from the powers they bestow' (1989, p. 118; 1997, pp. 41, 69, 245). A categorical property has a nature that is 'exhausted', or completely spent, in its instantiation, meaning that it does not reserve of itself for further interactions with other particulars (1997, p. 69). Bird describes Armstrong's categorical properties as having no 'nontrivial modal character', as not conferring essentially or necessarily any dispositional character (2005a; 2005b, p. 147). As for properties, likewise for relations between particulars, since they can be treated in much the same manner as properties, except that they are polyadic rather than monadic (Armstrong, 1997, p. 85). (In the case of relations that are universals, the Principle of Instantial Invariance holds.)

Just as universals can be first-order or higher-order (state of affairs types), so the laws - defined as relations between universals - also have fundamental and higher levels. For Armstrong, universals do not exist as Platonic Ideals, but only in their 
instantiation. Hence, at the fundamental level, relations between instantiated universals are singular instances. Even if these relations occur in regular, reliable patterns, there is nothing about the situation per se that accounts for the uniformity of nature, or implies its continuation, short of the existence of 'strong' laws (1997, pp. 82, 226). Although strong, however, the laws need also to be contingent. As Armstrong notes, contingency seems to follow from the self-contained nature of categorical properties (1997, p. 82). In the language of possible worlds, a description of strong but contingent laws would be those that provide necessity in the actual world although they need not be representative of the laws that hold in other possible worlds (1989, pp. 117-118). Explaining how contingent laws supply the necessity to underpin nomic uniformity is a major challenge for Categoricalism, and will be discussed in detail in Chapter 3 of this Thesis. Relations between instances of universals need something more in order to be posited as law. This 'something' is a necessitating relation (N), but just as the Contingent Identity Theory evolved gradually, so too has this necessity relation been subject to modification across the years.

Beginning in the 1970s Armstrong's necessitating relation $N(F, G)$ was presented as a first-order particular rather than a universal. By the early 1980s it was represented as a second-order particular and thus a first-order universal. The following passage explains Armstrong's reasons for the change:

In my 1978, I said that a state of affairs such as $\mathrm{N}(\mathrm{F}, \mathrm{G})$ was not a universal, but a particular (Vol. I, 115, n.1). I arrived at this view in the course of thinking about a very interesting phenomenon in the theory of universals which I called, rather melodramatically, "the victory of particularity"... Particulars + universal $=$ a particular. $I$ then argued, as an afterthought, that $\mathrm{N}(\mathrm{F}, \mathrm{G})$ was equally insusceptible of repetition. Even universals + higher-order universal $=\mathrm{a}$ (first-order) particular. States of affairs are always first-order particulars.

But perhaps the afterthought was a mistake. In the state of affairs, Rab, the first-order particulars, $a$ and $b$, together with the first-order universal, R, yield a state of affairs which is a first-order particular. In the state of affairs, 
$\mathrm{N}(\mathrm{F}, \mathrm{G})$, the second-order particulars, $\mathrm{F}$ and $\mathrm{G}$, together with the second-order universal, N, yield a state of affairs which, considerations of symmetry suggest, is a second-order particular. But a second-order particular is a firstorder universal... $\mathrm{N}(\mathrm{F}, \mathrm{G})$ would be a first-order universal, and its instances will be the positive instantiations of the law (1983b, pp. 89-90).

In the passage above, $\mathrm{N}$ is the necessitating relation linking $\mathrm{F}$ and $\mathrm{G}$, which are states of affairs types. Nonetheless:

Laws are categorical states of affairs - higher-order states of affairs - linking properties (states of affairs types) directly and issuing in regularities involving the particulars which have the properties... On this scheme, furthermore, the law has no existence except as it is thus instantiated in instances (1996b, p. 43).

To accommodate the fact of laws existing as relations between types but also not existing except in the instances, Armstrong considers laws to be both categorical relations between instantiated properties and also higher-order relations between types of states of affairs (1996b, p. 43): 'laws are connections of properties, or, alternatively but apparently equivalently, connections between types of states of affairs...These properties are universals' (1996b, p. 43). According to the above passage from 1983, a (categorical) relation linking two second-order universals such as $\mathrm{N}(\mathrm{F}, \mathrm{G})$ must be considered a second-order particular. But $\mathrm{N}(\mathrm{F}, \mathrm{G})$ is equivalently a relation, $\mathrm{R}$, between instances of the types $\mathrm{F}$ and $\mathrm{G}$, and thus constitutes a relation between fundamental particulars given as a first-order universal in Armstrong's scheme, e.g. Rab.

Armstrong establishes duality concerning the second-order particular $(\mathrm{N}(\mathrm{F}, \mathrm{G}))$ and first-order universal (Rab), by means of collapsing $\mathrm{F}$ and $\mathrm{G}$ as types to their representative instances. He explains as follows:

I propose that the state of affairs, the law, $\mathrm{N}(\mathrm{F}, \mathrm{G})$, is a dyadic universal, that is, a relation, holding between states of affairs. Suppose that a particular object, $a$ 
is $\mathrm{F}$, and so, because of the law $\mathrm{N}(\mathrm{F}, \mathrm{G})$, it, $a$, is also $\mathrm{G}$. This state of affairs, an instantiation of the law, has the form $\mathrm{R} a b$, where $\mathrm{R}=\mathrm{N}(\mathrm{F}, \mathrm{G}), a=a$ 's being $\mathrm{F}$, and $b=a$ 's being $\mathrm{G}$ :

$$
(\mathrm{N}(\mathrm{F}, \mathrm{G}))(a \text { 's being } \mathrm{F}, a \text { 's being } \mathrm{G}) \text {. }
$$

...I do not see why $\mathrm{N}(\mathrm{F}, \mathrm{G})$ should not simultaneously be a (dyadic) universal and a state of affairs (1983b, p. 90).

Here, $\mathrm{N}$ is a dyadic universal, the law that links the universals $\mathrm{F}$ and $\mathrm{G}$ (state of affairs types); but it is a state of affairs in the form $\mathrm{R} a b$ where $\mathrm{R}$ is a relation instantiated together with $a$ and $b$. Since $\mathrm{F}$ and $\mathrm{G}$ do not exist apart from their instantiations, $\mathrm{F}$ is seen in the token instance $a$ 's being $\mathrm{F}$, and $\mathrm{G}$ is seen in $a$ 's being $\mathrm{G}$, such that a relation between the universals $\mathrm{F}$ and $\mathrm{G}$ is also a relation between the fundamental instances. Thus, the higher-order law between $F$ and $G$ is represented by the fundamental relation $\mathrm{R}$ between $a$ and $b$, since the state of affairs $\mathrm{N}(\mathrm{F}, \mathrm{G})$ is the type instantiated by $\mathrm{R} a b$.

The reasoning in the passage above is problematic, however, at the step where Armstrong replaces ' $a=a$ being an F' with F itself; and likewise ' $b=b$ being a G' with $\mathrm{G}$ itself, and $\mathrm{N}$ with $\mathrm{R}$ :

$\mathrm{R} a b$, where $\mathrm{R}=\mathrm{N}(\mathrm{F}, \mathrm{G}), a=a$ 's being $\mathrm{F}$, and $b=a$ 's being $\mathrm{G}$ :

$(\mathrm{N}(\mathrm{F}, \mathrm{G}))(a$ 's being $\mathrm{F}, a$ 's being $\mathrm{G})$.

By 1997 Armstrong explicitly recognises that the difference of order between token and type - first-order versus second-order levels - demands different treatments, and that $F$ and $G$ had thus far been under-described:

It must be conceded that those of us who uphold the view that laws of nature are relations of universals have often used a symbolism... The connection was represented as $\mathrm{N}(\mathrm{F}, \mathrm{G})$ and the entailment of a universally quantified truth, a regularity, seemed a mystery. For N I am now substituting $\mathrm{C}$ for cause...But it was the "( $\mathrm{F}, \mathrm{G})$ " part of the formula that seriously underdescribed the situation (1997, pp. 228-229).

Being a type of particular, F cannot be 'untransparently' collapsed into the lower- 
order as Armstrong had supposed, since $\mathrm{F}$ is not, in fact, equivalent to $a$ 's being F; nor is $\mathrm{b}$ equivalent to $a$ 's being $\mathrm{G}$. But this very dual-status of $\mathrm{F}$ and $\mathrm{G}$ as both fundamental and higher-order was what had allowed Armstrong to have necessity of laws at both fundamental and higher levels.

Armstrong's 1997 answer is to dispense with the duality by reiterating that relation instances are instantiations of the nomic states of affairs types:

A law... is a causal connection between state-of-affairs types. It is a 'direct' connection between these state-of-affairs types, that is, between universals. It does not hold between universals via their instances...A reader who has been led through this [his 1997] argument, has first been presented with singular causation, and then has been asked to consider the suggestion that the same relation of causation which holds between suitable token states of affairs could also hold between state-of-affairs types. But as has but recently become clear to me, this is a ladder that can now be thrown away. The fundamental causal relation is a nomic one, holding between state-of-affairs types, between universals. Singular causation is no more than the instantiation of this type of relation in particular cases (1997, pp. 226-227).

Armstrong proposes a direct connection between what happens at the instance-level and at the type-level (1997, p. 228): 'Each case of singular causation is a relationship between first-order states of affairs, but where this relationship instantiates a law (or laws), a law that is a causal connection of state-of-affairs types (universals)' (1997, p. 227). Each time a causal relation between $a$ and $b$ occurs, it does so by instantiating a higher-order law.

This 1997 solution, discussed in more detail in Chapter 3, incurs a problem in so far as these higher-order laws do not exist except in their instances. Armstrong emphasises that although his laws might echo Platonic transcendent Universals or Ideals that 'govern' their instances, this is not what he envisages (1997, p. 226). Rather, he asserts that there is nothing to the law except what is instantiated, and draws upon the nature of universals, as repeatables, to justify an abduction about higher-level laws: if different instances involve the instantiation of an entity strictly 
repeated, then those instances imply the existence of a law that is more than merely an inductive generalisation (1997, p. 227). The nature of universality allows him to draw this abduction:

A regularity in nature is observed. It is postulated that behind this regularity, explaining it, and predicting further observations, is some connection of properties, some connection of state-of-affairs types...the unifying power of the explanation depends on the true and ultimate laws being connections of universals. Following Gilbert Harman (1965) I have in the past described this sort of explanation as inference to the best explanation, and am still prepared to do so (1997, p. 236).

This controversial formulation is the topic of considerable criticism in the literature, and a closer analysis is undertaken in Chapter 3.

\subsection{Summary and Conclusion}

This chapter has traced the development of Armstrong's Contingent Identity Theory of Dispositions within the context of Categoricalism. It began with an outline and brief contextual history concerning the type-type identity inherent in the early metaphysical identity of dispositions and the microstructure of their bearers. Reasons for positing such a reduction included needing to account for unmanifested dispositions. Problems with multiple-realisability enforced the recognition that tokentoken identity needed to replace type-type identity. Accommodating contingent laws in this latter development required this token identity to be contingent upon the laws of nature, the importance of which rests in providing the strong necessity that Armstrong builds into his system. Just as the Contingent Identity Theory of Dispositions represents an outcome of theoretical evolution, so the necessitating relation packaged with the laws evolved in response to philosophical debate. Analysis of the apparent bootstrapping that results will be undertaken in Chapter 3. 


\section{CHAPTER 3 \\ David Armstrong: \\ CATEGORICALISM - DisCUSSION}

The previous chapter outlined Categoricalism and introduced certain difficulties for the theory, the most challenging being that necessity is required for an adequate account of strong causation, yet laws as relations between states of affairs types do not exist over and above their instantiated instances. This chapter discusses these concerns in more detail and agrees with arguments criticising Categoricalism for its inability to demonstrate the necessity required for strong causation.

In section 2.1, I noted that one of Armstrong's criticisms of Ryle's position on dispositions concerned Ryle's inability to justify ascribing unmanifested dispositions. Since Ryle's dispositions are only contingently connected to the microstructure of their bearers, there is no reason why the connection must exist in the present, or indeed would continue to exist in the future. In contrast, Armstrong's early reductive theory was supposedly able to warrant the claim for the existence of unmanifested dispositions by identifying types of dispositions with types of microstructural bases. Similarities in types of microstructures justified inferences regarding similarities in their properties. Armstrong seemed to have the advantage of the canonical debate. But once Armstrong's position on dispositions changes from a metaphysical reduction to a supervenient token-token identification, it relies very heavily on the laws of nature to warrant the identification (1997, pp. 82-83).

Importantly, for Armstrong, the laws are contingent, existing simply as a 'matter of fact', the fact of the contingency ensuing from the self-contained nature of categorical properties (1997, p. 82). Such a view faces the challenge of explaining where the necessity required of strong causation, advocated by Armstrong, arises in a world of purely categorical properties and contingent laws. Categoricalism, being a 'soft theory of powers' (Armstrong, 2004b, p. 142), provides for the necessity via the laws of nature. Singular causation occurs between instances. Armstrong defines this as a certain state of affairs (the cause) bringing about a further state of affairs (the effect) (1997, p. 218) via law-governed singular causation. Being law-governed, 
singular causation is not 'mere regularity' (1997, p. 218). Since it is governed by relations between universals (repeatables), it is also nomic (1996c, p. 102). However, 'lawful singularity' is subject to a trilemma, as outlined by Armstrong (2004b, p. 128):

(1) Singular causation is a relation 'intrinsic to its pairs'(strong causation)

(2) Singular causation is essentially law-governed

(3) Laws are essentially general

If singular causation is intrinsic (1), then it is local to the relata. But, by (2) and (3), law-governance indicates that this local relation is part of a wider system, so it cannot be strictly local. The problem is that causal relations link particulars (locally), but laws link universals (non-locally).

Armstrong's solution draws on the repeatability of universals (properties) such that connections exist between instances as well as between higher-order state-ofaffair types (2004b, pp. 130, 133-134):

The idea here is to postulate a causal, or cause-like, connection not merely between particular states of affairs - this billiard ball hitting that billiard ball, and making the latter move - but also, at a higher-order level, between the universals involved in the first-order states of affairs... Now, consider truths involving general propositions: for instance, <the ingestion of a certain quantity of cyanide causes death $>$. Current philosophical thought wants to put this in the form of a universally quantified truth about particular events. But this is not its surface form. The surface form asserts a causal connection between kinds of events... My suggestion is that this is closer to the real form of a causal law. The law is a causal connection between these kinds. The same sort of connection which we have direct experience of at the level of particulars is here postulated (on good evidence) to obtain between the kinds themselves. A universally quantified truth can then be derived from this truth, analytically it would seem. But the universally quantified truth by itself is no more than good evidence for the direct causal connection of the kinds...all 
laws involve the instantiation of certain universals ensuring that the particular that instantiates them (or some further particular of a certain nature in a certain definite relation to the original particular) instantiates certain further universals (Armstrong, 2004b, pp. 133-134).

Whereas the 'surface' form describes connections between token events, the actual form is a connection between kinds of events, such that a law holding between states of affairs (instances) is really a causal connection between kinds (2004b, p. 134). Causal relations between instances of universals instantiate these higher-order laws but do not themselves constitute laws (1997, p. 227). Because instances are of kinds, we may infer the existence of laws via experience of the instances. But there is more than merely inference from instances to laws. Ontic relations between the kinds ensure the relations between instances, with such necessitation supposedly provided by the laws. This would seem to make the laws, as relations between kinds, responsible for necessity. Yet, these laws do not exist over and above their instances, which would seem to make the relations between instances responsible for the necessity required for strong causation.

The apparent bootstrapping effect that emerges in this theory is of concern to critics of Categoricalism, including Charles Martin, Herbert Hochberg and Alexander Bird, who each argue that Categoricalism cannot successfully derive the required necessity. Martin focuses his criticism on the idea that laws ultimately supervene upon the relations between instances. According to Martin, necessity is inadvertently introduced into Armstrong's Categoricalism via repeatability and thus connectability; these being ascriptions of irreducible dispositionality. Hochberg argues that reliance on laws as relations between kinds is untenable; and that passing responsibility back and forth between the instances and the kinds, although perhaps resulting in the appearance of necessity, actually fails to account for it. Bird points to the same regress identified by Hochberg, namely that a source of entailment requires higher and higher-order analogues of a necessitating relation, which is ultimately not provided. 


\subsection{Martin's Argument Concerning Connectability and Repeatability}

In Dispositions: A Debate (Crane et al., 1996) Armstrong describes the role of the laws thus:

Let us now apply such a scheme to the case where a brittle glass is struck, and as a result shatters. The striking of the as yet unbroken glass may then be thought of as the instantiation of a very complex universal which, because there is a certain forward linking of universals, brings forth the glass in a shattered state (1996b, p. 46).

This 'connecting' or 'forward linking' of universals indicates to Martin that Armstrong sequesters something 'in' the first-order properties. The grounding of dispositional properties in categorical properties depends heavily on: i) properties being repeatables; and ii) repeatables forming regularities in virtue of being repeatables. Because property universals are repeatables, the relations between their instances will form a pattern, the same relations between the same universal instances. As Armstrong notes, 'we can say that an F, simply in virtue of being an F, will bring forth a G' (1996c, p. 100). Martin (1996a, pp. 174-177; 1996c, pp. 127129) argues that Armstrong's laws are strong and thus capable of ensuring the connections between the instances; but that the necessity built into Armstrong's system cannot be accounted for in terms of purely categorical properties and relations. Although not explicitly recognised, there is irreducible dispositionality present in Armstrong's ontology. Given that: i) the same universals will be linked in the same way each time; and ii) these links are external to the first-order properties that are their relata, Martin asks what makes the same links instantiate between the same properties each time. The answer seems to be that the properties are repeatables; but this indicates that it must be something 'in the properties' themselves that affords them to link repeatedly the same way each time. Yet, it cannot be 'in' the properties unless the properties are not distinct from the laws, in which case they are not 'selfcontained', categorical properties. Thus, to rely on the repeatability of universals is to admit irreducible dispositionality into the ontology. 


\subsection{Hochberg's Criticism of N: Reliance on Ambiguity}

Armstrong's relation of natural necessity, 'N', is described by Hochberg as occurring where, 'a primitive higher-order causal relation between universals naturally entails corresponding specification' (1999b). When holding between two universals (or more correctly, two state of affairs types) $F$ and $G$, we write N(F,G). But as Hochberg notes, Armstrong sees this as a higher-order state of affairs (1999b, p. 485). Hochberg means that Armstrong's laws are relations both between token atomic states of affairs (i.e. 'this $F$ ' and 'this $G$ ') and simultaneously between types of states of affairs ('F' and ' $G$ '). But Hochberg denies the logical possibility of the latter case if F and G do not exist except in their instantiations. Armstrong writes that laws 'exist nowhere and nowhen except in their positive instantiations' (Hochberg, 1999b, p. 239). There can exist, in ontologically-robust terms, a totality of token states of affairs of particular kinds. However, no such corresponding ontologically-robust state-of-affairs type can exist if, as Armstrong proposes, laws exist only in their instances. The upshot is that there can exist an ontologically-robust relation between 'this F' and 'this G', but not between $\mathrm{F}$ and $\mathrm{G}$; the relation between $\mathrm{F}$ and $\mathrm{G}$ as types can be only an abstraction from the totality of relations between instances.

Hochberg notes Armstrong's claim that, because a relation between instantiated universal instances - higher-order or otherwise - is a state of affairs, laws are simultaneously both instantiated constituents of states of affairs and dyadic universals acting as 'functors', combining two universals to form another complex universal (Hochberg, 1999b, p. 485). For Hochberg, construing laws in both these ways is 'misusing' $\mathrm{N}$ by relying on its ambiguity to achieve an illusory goal:

Fusing these distinct roles of the causal connection N, Armstrong has N(F,G) as a fact, a Husserlian law of nature that is the ontological ground for 'all F's are G's' stating a causal law, as opposed to an accidental generality, and as a property that is exemplified by particulars. This ambiguous use of $\mathrm{N}$ enables him to achieve the specification to a first order generality and its instances. But, like Husserl, he can provide no account of the entailment involved in deriving the universal generalization (Hochberg, 1999b, p. 486). 
In truthmaker terms, we could take either a bottom-up or top-down approach. The bottom-up approach would let instances act as ultimate truthmakers for successive higher-order levels of instances and relations. In this case, a first-order relation, $\mathrm{F} a \mathrm{RG} a$, instantiating the law, $\mathrm{N}(\mathrm{F}, \mathrm{G})$, serves as truthmaker for its own necessity, since the law supplying this necessity supervenes upon these first-order instances. Relations between instances also act as truthmakers for the necessary relations between instances (and higher-order instances) of types. But it is unclear how regularities of instances can act as truthmakers for something stronger than mere regularity.

The top-down approach would allow that, although we infer the existence of types via instances, the same types ensure the existence of the relations between instances. This approach appears, superficially, to solve the lack of necessity in the bottom-up approach. However, as pointed out by Hochberg, understanding types other than as abstractions from the totality of instances belies the claim that laws do not exist over and above their instances. If ontologically-robust relations between types do not exist, then they cannot be posited as ultimate truthmakers for the supposed necessity of the relations between instances. This would require a regress of higherand higher-order instances of $\mathrm{F}$ and $\mathrm{G}$ to be truthmakers for lower-order relations, reaching no actual $\mathrm{F}$ and $\mathrm{G}$ existing apart from their instances. $\mathrm{N}(\mathrm{Fx}, \mathrm{Gx})^{10}$ relies on a higher-order necessity holding between instances of universal types $N(F, G)$, which relies on further higher-order necessity, passing on the burden of explanation ad infinitum (Hochberg, 1999a, p. 254).

Hochberg argues that Armstrong's apparent necessity is an illusion born of juxtaposing the bottom-up and top-down approaches (1999a, pp. 244-274; 1999b, pp. 486-488; 2001, pp. 299-317), producing a truthmaker for neither the relations between instances, independent of the higher-order laws; nor for types, independent of relations between instances. In Hochberg's view, the law instances, the law regularity, and the law necessity must be treated separately, rather than as a fused notion. This means separating $\mathrm{N}\left(\mathrm{Fx}_{\mathrm{x}}, \mathrm{Gx}\right)$ as a higher-order fact; $\mathrm{N}\left(\Phi_{\mathrm{x}}, \Psi_{\mathrm{x}}\right)$ as a higher-order relation between universals; $\mathrm{N}(\mathrm{Fx}, \mathrm{Gx})$ as a first-order universal that "contains" the relation $\mathrm{N}(\Phi \mathrm{x}, \Psi \mathrm{x})$; and $\mathrm{N}$ as a functor forming the universal from Fx and Gx (1999a,

\footnotetext{
${ }^{10}$ Hochberg does not italicise representation of particulars in the same manner as Armstrong.
} 
pp. 258-260; 1999b, p. 486; 2001, pp. 301-303). However, this separation would require amendments to Armstrong's laws. The next section draws on Bird's formal characterisation of Armstrong's laws and properties to argue that what kind of revision would be required is not be achievable within the framework of Categoricalism.

\subsection{Bird's Formal Characterisation}

Bird's argument follows from a summary of Armstrong LAWS (Armstrong, 1983b, 1997):

LAWS: Laws of nature are contingent relations among natural properties (Armstrong 1983). If $\mathrm{F}$ and $\mathrm{G}$ are first-order universals, then a law relating them is the fact of a certain second-order universal relating $\mathrm{F}$ and G. We may call that second-order relation ' $\mathrm{N}$ ', so that the law may be symbolized $\mathrm{N}(\mathrm{F}, \mathrm{G})$. $\mathrm{N}$ has certain properties. For example: $\mathrm{N}(\mathrm{F}, \mathrm{G})$ entails $\forall x(\mathrm{~F} x \rightarrow \mathrm{G} x)$. Let us call the relation between $\mathrm{F}$ and $\mathrm{G}$ that holds whenever $\forall x(\mathrm{~F} x \rightarrow \mathrm{G} x)$ the 'extensional inclusion relation', symbolized thus: R(F,G). So N(F,G) entails $\mathrm{R}(\mathrm{F}, \mathrm{G})$. However, $\mathrm{R}(\mathrm{F}, \mathrm{G})$ does not entail $\mathrm{N}(\mathrm{F}, \mathrm{G})$, since the relation of necessitation is not the same as nor coextensional with the relation of extensional inclusion. This is clear because there may be accidentally true generalizations without any corresponding law (2005b, pp. 147-148).

Bird's formal characterisation of Armstrong's N:

(I) $\langle\mathrm{N}(\mathrm{F}, \mathrm{G})\rangle$ entails $\langle\mathrm{R}(\mathrm{F}, \mathrm{G})\rangle$

where $\mathrm{N}(\mathrm{F}, \mathrm{G})$ is the relation of necessitation and $\mathrm{R}(\mathrm{F}, \mathrm{G})$ is the extensional inclusion relation between instances. (I) tells us that wherever there is a necessitating relation, there is a relation. A general law, Armstrong's relation between state-of-affairs types is just such a necessitating relation. The difficulty that such a necessitating relation raises is that it would seem to entail a nontrivial modal property. However, if all of Armstrong's properties are categorical, they cannot be nontrivially modal.

The problem might be remedied, suggests Bird, by dropping the entailment between the necessitating relation and the extensional inclusion relation, leaving Armstrong with only a regularity theory of laws rather than a causal theory of strong 
laws, which would be unacceptable to him. Alternatively, making $\mathrm{N}$ only contingently necessary would provides a kind of necessity, although disallowing entailment; dictating merely that a relation between this $\mathrm{F}$ and this $\mathrm{G}$ is necessary, given that the relation between $\mathrm{F}$ and $\mathrm{G}$ is necessary. However, this modification would seem to require an even higher-order relation to explain why the relation between $F$ and $G$ is necessary in the first place. Such a higher-order analogue of $\mathrm{N}$ would require still higher-order analogue ad infinitum (Bird, 2005b, p. 151). Hence we get the same regress identified by Hochberg.

\subsection{Quiddity-Minimalism}

The above criticisms focus on an explanatory lacuna in justifying necessity in a purely categorical world. This predicament does not occur for Necessitarians ${ }^{11}$ concerning dispositions, who hold that properties (or at least some properties) are intrinsically powerful and that laws merely describe the outworking of the essential natures of these properties. Categoricalism, however, purports that laws are prescriptive, rather than descriptive, in the sense that they play an active role as partial truthmaker in the identification of token dispositional properties with the token microstructures of their bearers. They play this role, presumably, in virtue of how they link properties. As already discussed, Martin questions why the linking process should not be attributable to something in the properties themselves, and thus represent irreducible dispositionality. Here I present a slightly different argument for why, given that Armstrong's laws are contingent and that the microstructural properties in question are categorical, it is doubtful that the Armstrongian laws would be able to link properties such that differentiations of power occur.

Armstrong holds a minimalist view of quiddity, for he allows properties to be interchangeable with respect to the powers they bestow. Robert Black describes 'quiddity' as whatever there is to a property apart from the power that it bestows on its bearer (2000). In keeping with this definition, Armstrong restricts quiddity of properties to bare numerical difference:

\footnotetext{
${ }^{11}$ These include Brian Ellis (2002), Ullin Place (1996b), Charles Martin (1993, 1996b), George Molnar (1999, 2003), Sydney Shoemaker (1984a) and Alexander Bird (Bird, 2005a).
} 
[C]onsidering a number of different simple properties, that is, properties that have no structure at all. These, on my view, would resemble each other in being monadic universals. How would they differ? They would differ numerically only! The parallel here is with particulars that exactly resemble and so differ numerically only...may we not argue that all simple monadic universals differ from each other purely numerically, all simple dyadic universals differ from each other purely numerically, and so on for all n-adic universals? (2000, pp. 19-20).

In 2000, Armstrong points out the difficulty faced by the traditional Categoricalist position on the nature of properties. In this view, properties were attributed an inner nature, yet could bestow different powers on particulars depending upon the circumstances. The problem with this view is in understanding what this inner nature might be, since it could be identified neither directly nor indirectly via the manifestation of a particular's power. Properties and relations are self-contained, 'distinct from the power they bestow' (1989, p. 118; 1997, pp. 41, 69, 245). Since properties are not essentially associated with their powers, then the powers pertaining to a property can be gained or lost across different possible world scenarios, just as the properties of a particular can be similarly gained or lost. As Armstrong notes, this seemed to render the inner nature of properties as mysterious and inaccessible (2000, p. 19). As Armstrong notes in 1997, a strong account of quiddity 'seems to involve ontological embarrassment', since the inner nature 'seems to elude the resources of natural science to deal with' (1997, p. 169). In avoiding the need to postulate quiddities, from 1997 onwards, Armstrong professes to a 'deflationary account of quiddity' according to which a difference between universals in the same adicity class (e.g. monadic, dyadic relation, triadic relation etc.) is merely numerically different in much the same manner as fundamental particulars are deemed to be (1997, p. 168).

In this formulation, no longer can the power that a particular bestows be associated with the inner nature, contingently or otherwise, of a property. However, in 1997 Armstrong also posits universals as being 'ways' that particulars are (Armstrong, 1997, pp. 122-124), and this is compounded by the claim that each universal within an adicity class can be identified with a different causal power it 
bestows (1997, p. 168). The change from a property possessing an inner nature, to being one that is merely numerically different from other universals in its adicity class, is at odds with the role of universals as providers of the qualitative character of the power of their bearers. Specifically, there appears to be a logical gap arising from the fact that if universals are 'the ways that particulars are', as Armstrong still describes them in 1997 (Armstrong, 1997, pp. 122-124), and yet they lack a nature that is qualitative, then no explanation is provided for the qualitative difference between manifested powers, or how properties contribute qualitatively to particulars such that they constitute those 'ways' described of them.

Among other reasons-including the difficulty of empirically addressing such an inner nature (1997, p. 169)-Armstrong's denial of a qualitative difference between universals is based upon an argument for the contingency of laws. In different possible worlds, the laws of nature might be different, and hence the very same universals could be associated with the manifestation of different powers. This view builds qualitativity into the laws themselves rather than into the instantiated universals. I argue, however, that since the laws are, according to Armstrong, relations between universal-types, if the universal types are only differentiated numerically, then there remains no explanation for how qualitative differences between powers arises. Neither is there an explanation for the relevance of defining universals as the 'ways' that particulars are. Since universality denotes repeatability, numerical difference must distinguish universal types rather than universal instances. But mere numerical difference between properties cannot explain how universal-type $\mathrm{F}$ should be considered to differ qualitatively from universal-type G, such that F-ness is different to G-ness. Consequently, it is not transparent how different microstructures give rise to different, specific powers.

Armstrong allows different possible worlds with different laws to uniquely associate microstructures with powers, indicating that the differentiation rests upon the laws. But if laws do not exist over and above relations between instances, and if these instances are differentiated only numerically, then it is unclear how laws may contingently differentiate one power from another, and hence identify any given disposition with a given microstructure. If laws were to represent relations between universal-instances, which all possess some essential nature besides numerical 
differentiation, then the way these entities are linked could give rise to differing qualities among microstructures. Without such differentiation of the nature of properties, however, it is not feasible for various ways of linking properties to afford qualitative differentiation of microstructures and the powers they bestow.

\subsection{Revisions Concerning Instantiation and Laws}

The contingency of Armstrong's laws can be expressed in possible-world terminology: The microstructure of an object may be identified with different dispositions across different possible worlds, depending upon which laws of nature prevail in such worlds. The idea, that it is the very same individual or object inhabiting distinct possible worlds, is referred to by Robert Black as 'Haecceitism' (2000, p. 92). This definition can be refined in light of the earlier paper by David Lewis on his discussion of Haecceitism. For Lewis, talk of possible worlds does not connote that the very same objects across different worlds exist, but rather, that we are referring to counterparts of objects, representing different possibilities within a single world. As he notes, 'I say: two possibilities, sure enough. And they do indeed differ in representation de re: according to one I am the first-born twin, according to the other I am the second-born. But they are not two worlds. They are two possibilities within a single world' (Lewis, 1986a, p. 231). Perhaps, however, the contingency in Armstrong's view aligns better with Bird's definition, that 'the transworld identity of particulars does not supervene on their qualitative features' (Bird, 2005a, p. 444). This indicates the identity of particulars being given in virtue of something over and above their properties. Taken strictly in this sense, Armstrong (1997) champions such 'moderate' haecceity, permitting particulars to possess transworld identity while denying that they have any essential nature except their particularity (1997, pp. 108109). (Note, haecceity was denied in Armstrong's earlier writing, and later revised.)

Although Armstrong prohibits the existence of bare particulars, his moderate view of haecceity appears to support at least the conceptual existence of thin particulars, which are defined precisely as individual particulars. Such thin particulars are never 'naked', however, and thus participate in states of affairs in which there are other qualities that determine the 'way' such states of affairs are. Armstrong claims these states of affairs to be primary and the constituents of states of affairs - namely 
particulars, properties and relations - to be dependent upon the existence of states of affairs itself (Armstrong, 1997, p. 29). Atomic states of affairs do not have parts in the sense of a mereological sum (1989, pp. 41-42). In the mereological case, while the sum would entail the existence of $a$ and F, $a$ and F could exist without the sum necessarily existing. But Armstrong's theory permits the existence of neither without their instantiation in a state of affairs (2004a, p. 144; 2006, p. 211).

What might be the nature of this instantiation? Contingent instantiation is conceptually troublesome. First, it implies that a particular may instantiate any given property, and any given property may be instantiated by any given particular. Thus, contingent instantiation reflects distinctness between instantiating particulars and properties, suggesting that somehow particulars and properties exist independently prior to their instantiation. Armstrong rejects both Platonic uninstantiated universals and un-propertied particulars, describing that their exemplification in states of affairs follows from their status as instances of higher order state-of-affairs types, at least in the case of properties. However, the regress problem concerning laws of nature, outlined in sections 3.1 to 3.3 , is also relevant in this case. If property-types explain the existence of property instances in states of affairs, these types must exist as more fundamental than states of affairs themselves. But this would lead back to their existence as some form of Platonic Ideal, a stance that Armstrong avoids. Yet, if property types exist only in their instances, this returns us to the question concerning the distinctness of properties from particulars as indicated by contingent instantiation.

A distinctness between the qualifying properties and the instantiating particular would also entail either some relation between them or another form of tie in order to link the two together in the post-instantiated state, in which case, an explanation of how instantiation is tied to its terms would be called for. Armstrong posits a non-relational but contingent tie (2004b, pp. 46-47). His reasons for rejecting relational ties involve a regress, described by F.H. Bradley, that obtains because for each relation tying the terms together, further relations are required to attach those relations, and so on (Baxter, 2001, p. 449; Bradley, 1897). Armstrong describes Bradley's regress in more detail: 
Suppose that $a$ stands in the relation $\mathrm{R}$ to $b$. $\mathrm{R}$ is instantiated by the pair $a$ and $b$. So a special extra relation of instantiation is needed to weld the ordinary relation $\mathrm{R}$ to the two particulars. And if it really is needed, then why is not a still further relation needed to get the special extra relation, and R, and the two particulars together, and so ad infinitum? F.H. Bradley and Quineans join hands here in a rather unholy alliance (1997, p. 114).

Since the relational tie incurs this regress, Armstrong proposes some sort of non-relational tie. As described in Section 2.3.2, Armstrong's arguments against particulars as merely bundles of properties borrowed Aristotle's notion of 'thissomething', and resulted in a theory of 'simultaneous unity and distinguishability' which captured Armstrong's contingent instantiation in the case of thick particulars (1978b, p. 110). Armstrong's fundamental but non-relational tie arises from states of affairs existing first; presupposing their constituents in the sense that 'there is no relation of instantiation over and above the states of affairs themselves' (1997, p. 118), although it also is a certain sort of fundamental 'structuring' of its constituents. How the constituents are organised determines what state of affairs there is, since two distinct states of affairs can have the same constituents but different arrangements of them. The identity condition for states of affairs resides in structure such that identical states of affairs will have the same constituents and organisation (Armstrong, 1997, pp. 121, 131-132).

Once structure is parcelled into the identity of states of affairs, its utilisation to explain the instantiation tie becomes incompatible with contingent instantiation, however. Donald Baxter argues that a non-relational tie involves a contradiction: 'Seemingly, if the things are distinct then the tie is a relation. If the tie is not a relation then they are not distinct. So a non-relational tie could hold between distinct things only if they are not distinct' (2001, p. 449). Armstrong's (2004) self-effacing solution is to introduce an in-between position that is neither strictly distinct nor indistinct: ${ }^{12}$

Suppose that a particular, $a$ as usual, has the non-relational property, good old $\mathrm{F}$, which is a universal...The difficulty is to understand the "fundamental tie"

\footnotetext{
${ }^{12}$ Here, Armstrong does not appear to be talking about a thin particular, but a thick particular.
} 
between $a$ and $\mathrm{F}$. The relation cannot be strict identity, because we want to distinguish the particular and its property. But equally, $a$ and $\mathrm{F}$ cannot be "distinct existences" because then they cannot be united except by a fully blown relation, call it "I" for "instantiation". Then, as F. H. Bradley and others have pointed out, the problem reappears. How are $a$, I and $\mathrm{F}$ to be brought together? One can, using Scotist terminology, speak of a "formal" distinction between $a$ and F, a distinction that is more than identity but less than a "real" distinction. But this, alas, seems a label for the problem, not a solution (2004a, p. 140).

Involving structure as one component builds-in the tie between properties and particulars such that it is an internal to the state of affairs itself, and therefore necessary. Recognising this — and also influenced by Donald Baxter's Aspect Theory which posits a partial identity between particulars and universals as the non-relational instantiation tie (Armstrong, 2005a, p. 317; Baxter, 2001)—Armstrong, by 2004, had come to regard instantiation as a necessary partial identity of the particular and its instantiated properties, rather than being contingent (2004b, pp. 46-49, 86). He adapts Baxter's partial identity to propose an Intersection Theory of instantiation (Armstrong, 2004a, p. 140). Imagining a table with the columns as particulars (thin) and rows as universals and properties, the intersections correspond to states of affairs (in the form of thick particulars). Rather than 'one running through many' whereby a particular instantiates possibly many properties, the table represents 'many running through many' (Armstrong, 2004a, p. 141). For Armstrong, these changes make instantiation necessary because, if particulars and universals were partially identical, any given particular could never lack any property that it had, and remain the same particular (2004b, p. 49; 2005c, pp. 317-318). In keeping with earlier assertions, Armstrong emphasises that in the aforementioned grid, the particulars and universals do not first exist, and then intersect to form states of affairs. Rather, they are 'illegitimate abstractions' (2004a, 143-144). If Fa is a grid intersection, then $a$ exists already — with all of its properties and relations - including F. Contrary to earlier claims, in 2004 Armstrong also posited the converse, that given F, $a$ also necessarily exists. However, Peter Simons and others (Armstrong, 2005a, 2006; Ehring, 2004; 
Rissler, 2006; Robb, 2005; Simons, 2005) have indicated problems for a world containing such very little contingency, and Armstrong rescinded this perspective in a reply to James Rissler (Armstrong, 2006).

Moreover, it can be argued that the moderate haecceity adopted by Armstrong in 1997, together with the claim that properties are merely numerically different (i.e. quiddity minimalism), problematises the idea of states of affairs being necessary, as Armstrong had suggested in the Intersection Theory. If transworld particulars have no essential nature except their numerical particularity, and if the properties that clothe such states of affairs in themselves represent no more than mere numerical difference in adicity, then different laws across different possible worlds would result in the very same states of affairs having different powers. This suggests a contingent rather than necessary instantiation of properties by particulars.

A further repercussion of the Intersection Theory involves letting the laws of nature be necessary rather than contingent $(2004 \mathrm{~b}, \mathrm{pp} .126,136)$. 'If this new theory of how particulars stand to universals is correct,' writes Armstrong, 'then the necessity involved seems to change the situation in looking for truthmakers in a number of controversial fields, for instance the status of laws of nature. It also involves rethinking the matter of states of affairs' (2004b, pp. 48, 52). He also writes that, 'In the past I have regarded external relations as holding contingently only, and I think that this has been the assumption generally made. But...this assumption can be questioned. Whether such relations are considered to be universals, or instead considered to be tropes, perhaps they should be thought of as necessary' (Armstrong, 2004 b, p. 52). Intersection theory proposes that universals and thin particulars intersect to form states of affairs. As Armstrong notes,

Particulars are ones running through many different universals, universals are ones running through many different particulars. A particular instantiating a universal is an intersection of the two sorts of oneness, a point of partial identity. That, on this theory, is what the formal distinction is. The particular and the universal intersect (Armstrong, 2004a, p. 141).

And:

If Baxter is correct, however, what we have in a fact or state of affairs is a 
non-mereological partial identity. Particulars and universals participate in each other. It is quite clear that this hypothesized partial identity of particular and universal is not mereological (Armstrong, 2004a, p. 141).

The states of affairs at the intersection points are not additional to the universals and thin particulars that intersect. Rather, as Armstrong describes, 'Given $a$ and given F...then the state of affairs of a's being $F$ is automatically there. It is built into the two constituents of the state of affairs' (Armstrong, 2004b, p. 49). Such a partial identity leads Armstrong to draw the conclusion that particulars and universals are thus an 'inseparable package deal' (2004a, p. 143), which, in turn, means for Armstrong that the state of affairs a's being $\mathrm{F}$ is necessary. He writes, 'the theory I now incline to: a's being $\mathrm{F}$ being necessary because $a$ and universal $\mathrm{F}$ intersect, and are thus partially identical' (2004b, p. 49). And again, 'Once given their constituents, states of affairs will be necessary, not contingent' (2004b, p. 51). Given that the universal types (i.e. laws) are nothing over and above the instances, then if the instances are partially identical with thin particulars, so too will be the universal types, with ramifications that these laws will be necessary:

[W]e should notice the possibility that the connections between universals involved in laws of nature are necessary rather than contingent... The relation of nomic connection, L (which I take to be best understood as a state-of-affairs type producing a further state-of-affairs type), will have to be partially identical with its universals, schematically F and G. It will then, I think, be a necessary connection, a necessity in re. If types are so connected, then the first-order particulars involved will conform to this linking of types $(2004 \mathrm{~b}, \mathrm{p}$. 136).

But if this partial identity leads to the laws of nature and causal relations being necessary connections between universals, it will surely affect Armstrong's view on dispositions; irreducible dispositionality in his theory might be unavoidable. As Armstrong replies to Bird: 
I think Alexander Bird has pointed to a real difficulty in my view of laws of nature, or at any rate the position I held in 1997 . He sees it as a clash between PROPERTIES: Natural properties have no essential or other nontrivial modal character; and LAWS: Laws of nature are contingent relations among natural properties. My properties are universals, and if some universals taken pairwise sustain such nomic relations then it appears that this sustaining is a nontrivial modal character of these universals. Some reconstruction seems necessary on my part. As a matter of fact I have, since 1999, become aware that my position on modality will have to change (2005b, p. 264).

Were the laws to be considered necessary rather than contingent, this would impact a great deal upon Categoricalism. First, properties could no longer be considered minimally quiddistic, since their essential nature would be given by what they do rather than what they are. If the laws, being relations between universals, are necessary, and if these universals do not exist over and above their instances, it follows that the laws do not exist except as relations between instances. But if the laws are necessary, and therefore not distinct from the instances they take as relata, then the instances must embody some essential nature concerning how the laws link them. In keeping with Martin's critique, this indicates an essential nature to properties, determined dispositionally by how they are linkable. Second, and positively, if the nomic necessity of the laws is built-in as a brute fact or irreducible dispositionality, Armstrong would have a viable explanation for the necessity that underpins strong causality. Third, instead of token dispositional properties and token microstructure being identified contingently according to the laws of nature, it would be the case that dispositional properties and the laws together were identified with the microstructure. But this scenario disallows properties being self-contained or complete in their instantiation, one of the hallmarks of categoricity. Rather, properties would be constantly 'giving of themselves' in the form of relations or links, and irreducibly dispositional.

For all of these reasons, the incorporation of necessary laws leads to Dispositional Essentialism, a view that is not compatible with the Categoricalism. Possibly for these reasons, and others, Armstrong has suggested in personal 
communication that he is now re-considering the 2004 revisions, wishing to maintain contingent laws. ${ }^{13}$ There is, however, benefit to be had by discussing the proposed 2004 revisions in terms of exploring available solutions to the problems that Categoricalism faces. It turns out to be extremely difficult to resolve the problem of explaining where a purely categorical world gets the necessity to shore up a strong theory of causality. This suggests that strong causality goes hand in hand with a theory of irreducible dispositionality. It does, however, tell us nothing about the compatibility of a Humean regularity theory of causality and Categoricalism, an issue that is beyond the scope of this Thesis, although a very interesting topic for future research.

\subsection{Summary and Conclusion}

The theory of strong but contingent laws required by Armstrong's Contingent Identity Thesis has proved to be problematic in terms of providing necessity, as discussed by Martin, Hochberg and Bird. The contingency of the laws also entails a distinction between properties and particulars, and thus contingent instantiation of qualifying properties by particulars in states of affairs. In Armstrong's view, properties do not exist except in their instantiations. However, contingent instantiation requires that properties exist independently of instantiation. A second difficulty with proposing properties and particulars to be distinct is that of explaining the instantiation tie. Relational ties confront Bradley's regress; a non-relational but contingent tie is presently without further explanation; and attempts to provide an account by proposing a partial identity of properties and particulars, as does the Intersection Theory, results in a slippery slide to necessary states of affairs and thereby, necessary laws. Moreover, this latter position can no longer be construed as a Categoricalist Theory of properties.

The lesson is that claiming all properties to be categorical seems incompatible with strong causality, regardless of whether laws are postulated to be contingent or necessary. Contingent laws are external to their relata, requiring further explanation

\footnotetext{
${ }^{13}$ In personal communication with David Armstrong at the 2007 and 2008 Australasian Association of Philosophy Conferences (AAP), he suggested that these changes were still very much in the formative stages and tentative. In conversation at the 2009 AAP, he mentioned possibly revoking some of these changes.
} 
for regularity of the relations between instances. This cannot be provided in terms of higher-order types without postulating an end-point, such as the transcendent Platonic ideal type, which is not legitimately categorical. Neither can regularity of the relations between instances be explained in terms of the relations themselves, since these comprise purely singular causation. Laws that are exemplified by necessary relations between instances would have truthmaker support in virtue of the necessity proposed at the instance-level. However, necessary relations could hold only by virtue of irreducible dispositionality among the relata, ruling them out as categorical. Regardless of whether laws are considered contingent or necessary, Categoricalism fails to accommodate the necessity required of strong laws. 



\section{SECTION 2:}

\section{STRONG DisPoSITIONAL ESSENTIALISM}

Section 1 showed that strict Categoricalism, since it does not adequately account for the necessity underpinning strong laws of nature, and since it relies upon these laws as partial truthmakers for dispositional properties, fails to give a satisfactory account of dispositionality. An attempt to account for the necessity that underpins a strong account of causation has encouraged some philosophers to favour Dispositional Essentialism (DE), and in so doing to defend the existence of irreducible dispositional properties. DE presents in various forms, including weak DE and strong DE. Weak DE theorists, for example Brian Ellis (2001a, 2001b, 2002, 2005b), merely acknowledge some properties that are essentially and irreducibly dispositional. Weak DE will be covered in Section 3.

This section presents and discusses Sydney Shoemaker's early ontology as a means of exploring some of the issues that arise for a Strong DE view. ${ }^{14}$ The Strong Dispositional Essentialist view is most often associated with the claim that no ontologically-robust categorical properties exist. This position is compatible with, but does not entail, a stronger claim that all properties are dispositional. This stronger claim is that which is held by Shoemaker in his early writing, and by Alexander Bird (2005a, 2006, 2007). The challenge for Strong DE, is to say how, without the existence of categorical properties, properties are individuated and recognised; how relations between distinct objects can be necessary; how the circumstances for the manifestation of power can be specified; and finally, whether the existence of power is discrete from its manifestation. It will be argued that addressing these problems would require the Causal Theory of Properties to be formulated either in terms of a Foundational-Monist theory, or else retreat to a view that incorporates fundamental categorical properties. The 1998 revision that Shoemaker introduces takes the latter path, disqualifying it as a pure-power theory.

\footnotetext{
${ }^{14}$ In 1980, Shoemaker's Causal Theory of Properties was published in two papers: A short paper entitled Properties, Causation and Projectibility (1980b); and a more detailed paper, Causality and Properties (1980a). This second paper was also published along with other seminal papers by Shoemaker in 1984 and in 2003 (1984a, 2003). I take my references to this second paper from the 1984 reprint.
} 


\section{CHAPTER 4 \\ SYDNEY SHOEMAKER: \\ Causal Theory of Properties - Outline}

In his early papers, Shoemaker draws a distinction between 'genuine' and 'mereCambridge' properties. ${ }^{15}$ The distinction is made in terms of causality (1984a, p. 209). Genuine properties of an object are identified by their abilities or potentialities for contributing to that object's causal powers (1980b, p. 294). A property that does not so contribute is a 'mere-Cambridge' property. Examples include: the property of being 'grue' (i.e. the property of being either blue or green dependent upon when the object is first observed (Goodman, 1973, p. 123)) and historical properties such as being twenty years old and having been slept in by George Washington (1984a, p. 208). Shoemaker also includes spatiotemporal properties, such as, 'being fifty miles south of a burning barn (1984a, p. 208) and other relational properties (e.g. 'Jimmy Carter is President of the United States') as mere-Cambridge (1984a, p. 208).

How might we verify which properties and relations are genuine and which are mere-Cambridge? Shoemaker notes that a property or relation is genuine just in case the causal powers to which it contributes can be ascribed to the object to which the property belongs. In the case of a distance or location, this supervenes (my terminology) upon the objects that stand in that distance relation. In this case, the properties of the objects themselves which stand in such a relation are genuine, but that the distance itself is mere-Cambridge:

[I]n the case of mere-Cambridge properties some of the operative causal powers will either belong to something other than the object to which the property is ascribed, or will belong to that object at a time other than that at which it has that property. Thus if I verify that a man has the property of being fifty miles south of a burning barn, it will be primarily the causal powers of the barn, and of the intervening stretch of land (which, we will suppose, I

\footnotetext{
15 The distinction between genuine and mere-Cambridge also applies to change, similarity and difference, as well as to properties (Shoemaker, 1980b, pp. 208-209).
} 
measure), rather than the causal powers of the man, that will be responsible for my verifying observations (Shoemaker, 1984a, pp. 220-221) .

In a further example, Shoemaker describes that genuine properties and relations contribute to intrinsic power, whereas mere-Cambridge power operates merely as a result of those genuine properties of objects. If an object's power can be changed without a change in its genuine properties, that power is a mere-Cambridge power, and arises in situations in which objects stand in relation to one another. $\mathrm{He}$ writes:

It is worth observing that there is a distinction between kinds of powers that corresponds to the distinction, mentioned earlier, between genuine and mereCambridge properties...A particular key on my key chain has the power of opening locks of a certain design. It also has the power of opening my front door. It could lose the former power only by undergoing what we would regard as real change, for example, a change in its shape. But it could lose the latter without undergoing such a change; it could so do in virtue of the lock on my door being replaced by one of a different design. Let us say that the former is an intrinsic power and the latter a mere-Cambridge power. It is clear that in my account of properties the word 'power' must refer only to intrinsic powers. For if it refers to mere-Cambridge powers as well, then what seems clearly to be a mere-Cambridge property of my key, namely being such that my door has a lock of a certain design, will make a determinate contribution to its having the powers it has, and so will count as a genuine property of it. But it seems unlikely that we could explain the distinction between intrinsic and mereCambridge powers without making use of the notion of a genuine change and that of a genuine property (Shoemaker, 1984a, pp. 221-222).

Thus relational properties involving causal relations and spatiotemporal relations between distinct objects are mere-Cambridge relations for Shoemaker. Things may have innumerable mere-Cambridge properties and relations, but these supervene upon the genuine, intrinsic, causally contributing ones. 
In 1998, Shoemaker introduces a change implying quiddity in his ontology. This chapter outlines Shoemaker's original position and this later change.

\subsection{Genuine Causal Properties}

Shoemaker's genuine causal properties (and relations) can be summarised in five main postulates:

i) Each genuine property is a set of causal potentialities, which are nomic, essential, primitive and immutable, unchanging across time or possible worlds (Shoemaker, 1980b, pp. 294-296; Shoemaker, 1984a, pp. 217-221). Here each set of causal potentialities is identified with a property.

ii) Properties contribute to the conditional powers of their instantiating objects. Contingent upon their co-instantiation with other properties, the instantiating object will possess a 'cluster' of conditional powers (Shoemaker, 1984a, p. 213). (Some, for example, Anjan Chakravartty (2003) and George Molnar (2003) refer to these conditional powers as the dispositions which an object possesses. Shoemaker, however, prefers to draw a verbal distinction between the term 'disposition' and 'power' with the former representing linguistic usage and the latter referring to power in an ontologically-robust sense (1984a, pp. 210-211).)

iii) A cluster of conditional powers is also identified as a property, albeit at a higher-order than a property comprised of a set of causal potentialities (Shoemaker, 1984a, p. 213).

iv) The identity of genuine properties is given by their causal contribution to the conditional powers of their bearers, such that it is not possible for two different properties to possess exactly the same set of causal potentialities (Shoemaker, 1980b, pp. 296-297). If properties X and Y make exactly the same causal contribution to an object, then $\mathrm{X}$ and $\mathrm{Y}$ are the same property (Shoemaker, 1998, pp. 64, 212). This identity applies across possible worlds (Shoemaker, 1984a, p. 221). In response to an objection raised by Richard Boyd (Shoemaker, 1984a, postscript), Shoemaker adds an additional requirement, that for $\mathrm{X}$ and $\mathrm{Y}$ to be the same property, the circumstances 
required for the instantiation of $\mathrm{X}$ are sufficient for the instantiation of $\mathrm{Y}$, and vice versa (1984a, postscript, p. 233).

v) While an object has a set of conditional powers, certain of these powers may manifest in particular ways depending on extrinsic circumstances involving external relations between the object and others. As noted already, such external relations are mere-Cambridge.

The identification of properties with sets of causal potentialities and clusters of conditional powers - even at differing levels in terms of higher- versus lower-order properties - results in a reduction of properties to powers. This is the main focus of Richard Swinburne's criticism of the Causal Theory of Properties (introduced in Chapter 1, and discussed here shortly). Swinburne's concern is that reducing properties to powers results in a pure-power world bereft of anything 'substantial'. This is also in response to Shoemaker's idea that genuine properties are causally potent while all other properties are supervenient, in which case properties, both as sets of causal potentialities and as clusters of conditional powers, cannot be individuated from each other. Individuation of properties would seem to require quiddity, something to properties over and above their causal power. These issues raised by Swinburne, along with neo-Swinburne arguments, are discussed more thoroughly in Chapter 5.

In a later paper, 'Causal and Metaphysical Necessity' (1998), by way of answering the Swinburne and neo-Swinburne objections, Shoemaker modifies his earlier position of the identification of properties and powers. This revision, discussed in more detail in Chapter 5, includes retrospectively denying that properties should be reduced to anything more fundamental (1998, pp. 63-64). Accordingly, the claim that a property is a cluster of conditional powers is revoked, and sets of causal potentialities are no longer identified with properties, but instead become backwardand forward-looking 'essential features' of properties.

\subsection{Bearers of Properties}

As noted already, the cluster of conditional powers is possessed by a bearer or instantiator of properties. This raises questions concerning the nature of individual 
entities. Two prominent characterisations are available: either an individual just is a bundle of properties (in this case each property being a cluster of conditional powers); or it is a thing which possesses a bundle of such properties. The latter is described by Michael Redhead as involving 'transcendental individuality (TI)', a phrase indicating the individuation of entities by some "essential "thisness", or haecceity, that transcends its properties' (Redhead, 1988, p. 10). As discussed in Chapter 3, haecceity can be viewed in terms of whatever there is to an individual over and above its properties. Clearly, a theorist advocating an individual as a bearer of properties incorporates haecceity into their theory. The bundle theorist may or may not accept the inclusion of haecceity, depending upon their view of how bundles cohere to form individuals, and whether the 'glue' or operation performing this task constitutes haecceity.

Shoemaker's early writing does not appear to make his stance on haecceity explicitly clear. However, he does adopt Jaegwon Kim's concept of 'constituent objects' (Kim, 1973; Shoemaker, 1984a, p. 206), affording the existence of distinct individuals related by contingently existing, extrinsic mere-Cambridge relations (Shoemaker, 1984a).

\subsection{Necessary Laws}

The Causal Theory of Properties regards the laws of nature as necessary, and holds that causal necessity amounts to metaphysical necessity (1984a, p. 226). As Shoemaker describes it, his account covers relations as well as properties, and the laws specify the relations between dispositional properties such that they comprise the intrinsic powers of objects, as well as the relations between objects and their properties:

This account could be extended to cover relations as well as properties. The laws that specify the causal potentialities of properties will frequently say what happens when things having certain properties are put in certain relations to one another; so these laws can be thought of as specifying the causal potentialities of these relations as well as the causal potentialities of the properties (Shoemaker, 1980b, p. 296). 
Genuine properties are 'marked off' from mere-Cambridge properties by their relations to causal laws (1984a, p. 217), which both govern the instantiation of properties and, by deriving from property instantiation, also specify the set of causal potentialities that constitute the essential natures of properties (1980b, pp. 299-300). Thus, laws both denote what genuine properties are, and also derive from these properties. While Shoemaker only retrospectively_via his 1998 revision-recognises a problem in actually reducing properties to powers and vice versa, his early writing explicitly acknowledges and permits the circularity involved in explanations of the relation between properties and powers. Indeed, exploring explanations of the relation between properties and powers seems to require an assumption of their non-identity. Shoemaker allows the explanatory circularity because he sees it as informative rather than vicious: 'As I see it, the notion of a property and the notion of a causal power belong to a system of internally related concepts, no one of which can be explicated without the use of the others' (1984a, p. 222). He considers that his view of necessary laws provides a genuinely explanatory account of how a thing retains the same causal powers over time: same properties, same powers (1984a, p. 216). Were a thing's genuine properties to change, then it would undergo a change in its power; justifying inference from particular instances of behaviour to general rules.

If properties are identifiable by their causal potentiality, as Shoemaker claims, this leaves the question of how spatiotemporal and other mere-Cambridge properties and relations are detected. How, for example, is the relational property of ' $x$ being fifty miles south of a burning barn' recognised, if it does not causally contribute to the powers of $x$ or of the barn? The general solution offered by Shoemaker is that all genuine existents form an aggregate by which mere-Cambridge properties and relations are inferred. In this particular example, the entirety of genuine properties includes the ground that physically lies between $x$ and the barn (1984a, pp. 220-221). Thus $x$, the burning barn and the stretch of land, act altogether as truthmakers for the ascription of the spatiotemporal distance between $x$ and the barn. Likewise for historical properties. Thus, the intrinsic causal properties of the multiple entities involved in any given system afford the detectability of mere-Cambridge properties and relations. These mere-Cambridge properties and relations could, therefore, be 
construed as higher-order properties that supervene upon the ontologically-robust existents of the causally potent world.

\subsection{Summary and Conclusion}

In this chapter I have briefly introduced and outlined Shoemaker's Causal Theory of Properties, which distinguishes between genuine and mere-Cambridge properties and relations, offering causal criteria as instruments of differentiation. Mere-Cambridge properties and relations supervene upon genuine ones. A genuine property is comprised of a set of causal potentialities, and each property bestows power on its bearers by contributing to a cluster of conditional powers possessed by the bearer. Complicating matters, Shoemaker also identifies the cluster of conditional powers with higher-order properties. Thus, the cluster of conditional powers is deemed to be a dispositional property which can then manifest in various ways depending upon the external circumstances of the bearer. Identifying properties with sets of causal potentialities reduces properties to something more fundamental and irreducibly dispositional. Likewise, identifying properties with clusters of conditional powers reduces properties to powers. The resulting pure-power world is the focus of Swinburne's regress criticism. In a later (1998) revision, Shoemaker attempts to avoid such a reductionist ontology by revoking the identification of properties with clusters of powers, and also by considering properties to possess rather than be comprised of causal potentialities. 


\section{CHAPTER 5 \\ SYDNEY SHOEMAKER: \\ Causal Theory of Properties - Discussion}

This chapter discusses Shoemaker's (1980) Causal Theory of Properties, beginning with a discussion of the Swinburne regress and associated calls to include fundamental categorical properties. This is followed by a discussion on George Molnar's critique of the dual criteria for defining genuine properties; as causally contributing and intrinsic to their object-bearers. I show why Molnar succeeds in his argument that spatiotemporal relations play a causal role in the moderation of intrinsic powers of their bearers; and contend that this presents a problem for the Causal Theory of Properties only in virtue of the theory incorporating distinct objects. Sidestepping this problem leads to a unification regress that must result, ultimately, in a foundational-monist theory, the alternative to which is the adoption of quiddity. This latter is the path that Shoemaker's 1998 revision takes, but it leads to a theory that no longer offers a pure-power view.

\subsection{Swinburne's Regress}

For early Shoemaker, properties are comprised of sets of causal potentialities that contribute to the cluster of conditional powers that a bearer of properties possesses. Each cluster of conditional powers also comprises a property, the nature of whose manifestation-effects is determined with respect to other co-instantiated properties, and prevailing contingent circumstances. Richard Swinburne pointed out that the conditional powers of objects are ascribed to them based upon the effects that objects produce when they manifest their powers. If properties are reducible to causal powers and potentialities, and hence to effects alone, the resulting pure-power world is one in which only effects exist, and to which there is nothing except other effects. The overall argument, and neo-Swinburne arguments that followed, speak to both ontological and epistemological considerations. The following passage outlines Swinburne's case against Shoemaker's Causal Theory of Properties: 
[T]he only good grounds for attributing causal powers to objects are in terms of the effects which objects typically produce. Such effects can only be recognized if properties can be recognized. But if properties are nothing but potentialities to contribute to causal powers, one will only have good reason for attributing them to objects if their effects can be recognized. This leads to a vicious infinite regress...Claims to recognize powers and so potentialities to contribute to powers, need justification in terms of the effects which objects typically produce, and that involves justification in terms of the presence or absence of properties. But if properties are nothing but potentialities to contribute to powers, once could only justifiably attribute such properties to objects if one had observed their effects. And so ad infinitum... (1980b, pp. 313, 317).

We recognise powers by their effects, but we can only recognise these effects by recognising properties involved in them. Object 1 produces effects on object 2, but since no genuine categorical properties exist, the effects on object 2 are recognised only in terms of the previous and subsequent properties of object 2. But such properties of object 2 are recognised only in terms of the effects that it produces on some object $3 \ldots$ and so on. The alleged problem is that the 'properties' are never actually encountered, since each one is represented by proxy as nothing more than effects. The only way that this regress can be broken, submits Swinburne, is if there is something more to properties than powers (1980b, p. 317).

The Swinburne regress can be framed in terms of a requirement for quidditycharacterised as whatever there is to a property over and above its causal power. Swinburne indicates that this might be whatever allows properties to be 'straight-off' available to our perception (Swinburne, 1980a, pp. 317-319):

For myself I do not hold that in any sense we often observe straight off the properties of our sensations, and certainly I do not hold that that is all we observe. What normally stares me in the face is not that something looks red, but that it is red; not that it feels square but that it is. So the redness and squareness of objects are among the properties which we observe straight 
off...If these are the redness and squareness of physical objects, rather than properties of sensations, we can see just by looking what more there is to such properties than the causes which produce them and the effects to which they give rise (p. 318).

Swinburne's claim is that when we perceive a property (or at least some properties), we do so because the property is in the object rather than being merely the property of some sensation that occurs in us. This is an argument for Lockean primary properties such as shape, size and so on (Locke, 1924, II, Ch. VIII, p. 66), properties that have been described as categorical. Swinburne's argument that categorical properties are those that can be observed 'straight off' is reflected in Brian Ellis's idea that categorical properties are required for 'direct perception' of properties to occur (Ellis, 2008a, 2008b). We may infer via other properties that a certain property exists, and this may continue in a chain of inference. However, somewhere along the line, the regress will need to be halted by direct perception. In essence, Swinburne's criticism of pure-power theories is that effects are imperceptible without the presence of categorical properties, which afford direct detection of the contents of the manifest world.

Alexander Rosenberg's (1984) argument from poverty of knowledge also concerns itself with detection of properties, although taking a slightly different tack in criticising the Causal Theory of Properties. Rosenberg claims that since it identifies properties by their relations, Shoemaker's theory requires a complete knowledge of the laws of nature before we can identify properties; and since having such complete knowledge is impossible, it must be impossible to identify properties (Rosenberg, 1984, pp. 81-82). Anjan Chakravartty points out that Rosenberg's argument is flawed in its assumption that a complete knowledge of laws is required to pick out or identify properties (2003, p. 396). He notes that partial knowledge has not, in the past, been problematic in gaining knowledge of certain properties of things in terms of specific relations. For example, we can use microscope slides to detect similarities among samples without knowing how all of the optical laws work, or we can measure the mass of an object without knowing all of the relations of which it is capable (2003, pp. 396-397). 
Shoemaker's answer to Swinburne flows in a similar vein to Chakravartty's response. While Swinburne interprets direct perception of effects as evidence for primary properties, Shoemaker allows that directly perceiving properties is made possible because perceivers are links in the chain of effects of these properties. Thus we can infer the existence of some properties and we can directly perceive the existence of some properties, but reaching an endpoint is not required for effects to be perceived, and indeed, when we observe or perceive a thing, we are being causally influenced by it in some way (1980b, p. 323; 1984a, p. 214). Chakravartty also notes that 'the buck stops with perception' because we participate in attributing properties to things; but whether effects carry on beyond the point of our attribution-making is not relevant (2003, pp. 397-398). Bird makes a similar point regarding direct perception, noting that one might be in the state of 'knowing' without necessarily perceiving further details of that state, such as, for example, that one is in that state. Bird says that this stops the regress because 'knowing' at any point in the chain of effects may allow knowledge of the preceding point in the chain and so on (Bird, 2007, p. 134). As Shoemaker notes, while we may infer the existence of properties by effects, we can also directly perceive some properties because we, ourselves, are involved in the chains of effects of these properties (1980a, p. 323).

According to Charlie Martin, John Heil and other proponents of neoSwinburne arguments, what is missing in Shoemaker's Causal Theory of Properties is not an ability to directly perceive properties, but an explanation of how objects might be perceived in a world devoid of properties capable of giving rise to our qualia (i.e. qualitative properties). In line with Swinburne's thinking, Martin asserts that there is something more to knowing or believing, something non-mental which underlies our knowing or believing. He writes, 'When we consider the need for physical qualia (that is, qualities), even in the finest interstices of nature, largely unregarded and unknown, among them should belong the qualia (qualities) required for the sensing and feeling parts of physical nature' (1997, p. 223). When we experience qualia, there is something ultimately responsible for us being caused to experience, over and above the fact that we experience. Behind any experience is a 'causer', and one must assume that this 'causer' cannot be, itself, pure effect, but rather, includes the qualitative properties or qualities of things. 
Since, according to the Swinburne and Martin arguments, primary or qualitative properties are a prerequisite for perception, their existence must precede perception and be therefore autonomous with respect to our senses. The difficulty with this claim lies in justifying how we would detect such sensory-independent properties. How might we detect redness or squareness 'straight off' rather than indirectly inferring their existence because of data supplied to us via our sensory modalities? George Molnar describes qualia as very compelling, immediate, urgent and intrusive, forcing us to accept the existence of at least psychological qualia (2003, p. 178). When we experience properties, therefore, it is understandable that we infer their independence in the objects that possess them, accounting for the experience of psychological qualia (or qualities) by the existence of physical qualia (or qualities).

However, the assumption that our experience of objects via their properties requires the ontologically-robust existence of categorical properties is not transparent. Molnar suggests that the ontological status of physical qualia can be readily questioned because what we experience cannot be the fundamental constituents of matter. Thus 'straight off' perception fails as evidence of fundamental categorical properties. Perception being ampliative rather than direct, we merely infer fundamental physical qualia or qualitative properties (2003, p. 178).

Were it the case that qualitative properties exist, might they exist at a higher level rather than as fundamental properties? Then the argument would be for a theory according to which categorical properties emerge from a pure-power base. I argue in Chapter 14 why I do not think this presents a serious problem, so long as these are merely supervenient, and by the Ontological Free Lunch Principle, not considered to be ontologically-robust. However, John Heil, in discussing Brian Ellis's New Essentialism (Ellis, 2001b, 2002; Heil, 2006, p. 42) argues that such a concept is incoherent. ${ }^{16}$ Martin also argues that a theory excluding fundamental physical quantities, or categorical properties, is left with an empty 'Pythagorean' ontology that, while allowing for something like properties as 'mere mathematicized measures', leaves out what those numbers or quantities exist in virtue of, namely qualitativity

\footnotetext{
${ }^{16}$ I discuss New Essentialism in more detail in Section 3 of this Thesis. Since Weak DE theories such as New Essentialism do incorporate fundamental categorical properties, I do not think the question is relevant here, although it does apply to the Causal Theory of Properties as presented in early Shoemaker and the Swinburne and neo-Swinburne arguments.
} 
(Martin, 1997, p. 195; Quine, 1976). The point is that Swinburne and neo-Swinburne arguments against the Causal Theory of Properties object to a world in which powers cannot be grounded in categorical properties. This would seem to indicate that the claim is one for categoricity at fundamental levels.

In response to Martin, Molnar suggests that numbers and quantities can just as easily be denotations of force or dispositionality as indicators of something qualitative (2003, p. 179). Martin's belief that a non-qualitative world is empty is tied to his view that the world must be considered in terms of 'space-filling' qualities such as shape and size, which he claims are needed to provide the 'shells' of entities (1997, p. 222) by which we 'sense and feel' physical qualia (1997, p. 223). However, as Molnar argues, space-filling applies only to the macro-world. Fundamental particles may have neither shape, size, nor volume; but are simply in space in virtue of having a spatial location (2003, pp. 175-176). Properties such as the size and shape of objects, therefore, cannot be said to represent experience of fundamental qualitative properties. Moreover, these properties are explanatorily idle in terms of fundamentality. For example, we do not need to explain the behaviour of an electron with reference to anything other than its powers. Its occupation of space needs no account in terms of qualities; and if it did involve qualities, they would add nothing of value over and above explanations already given in terms of the dispositions of an electron to behave in certain ways. Hence, Molnar argues, we tend to treat qualities as ontologically respectable even though they are 'unobservable and idle'. He further suggests that if we have 'neither experimental nor theoretical evidence' for believing in something, then we should not do so (2003, p. 178).

Molnar also argues against John Foster's (1982, pp. 66-72) claim for the existence of categorical properties in terms of the requirement for space-occupiers, which Molnar says incorrectly amounts to a claim for space-filling. Foster proposes that it is inadequate to conceive of power as that which 'affects or is affected', because to do so is to leave power unspecified. Rather, power imbues its bearersome object — with the ability to affect or be affected. Setting up a hypothetical world of atoms that are nothing but spheres of impenetrability, Foster argues that to answer a question demanding the specification of a power with a description of the effect of 
that power is inadequate because attempting to specify powers in terms of other powers leads to a vicious regress:

The problem arises when we ask: 'To what is a sphere of impenetrability impenetrable?' The answer is: 'To other atoms, i.e. to other spheres of impenetrability.' But this means that the specification of the content of the atom-constituting power is viciously regressive: each atom is a sphere of impenetrability to any other sphere of impenetrability to any other sphere of impenetrability.... and so on ad infinitum. From which it follows that the notion of such a power is incoherent, since there is nothing which the power is a power to do. To conceive of a sphere of impenetrability, we have to postulate some other type of space-occupant whose passage it is empowered to obstruct (1982, p. 68).

This argument suggests that a coherent account of power cannot be given in terms of other powers, since no real specification is thereby achieved. We may recall Nietzsche's reference to Molière's doctor who was asked, 'How does opium induce sleep? And he replied, 'quia est in eo virtus dormitiva, cujus est natura sensus assoupire', which means, 'because it contains a dormative virtue whose nature is to put the senses to sleep' (Holbo; Nietzsche, 1886/1923, section 11). As Keith Campbell notes, we are asking for the power, as effect, to be explained, but are answered with only a description of the effect in question (1990, pp. 117-119). 'The only way of avoiding the regress it seems', writes Foster, 'is to construe at least one of the powers as a power to affect the behaviour of some type of substantial spaceoccupant - an occupant with an intrinsic nature independent of its causal powers and dispositions' (1982, p. 69). He thus rules out the idea that power can affect or be affected by power, because space-occupants are required as the objects of the effect of power. But, as Molnar points out, it is a false assumption that only objects with a nondispositional essence can occupy space (2003, pp. 173-174). He sets out the Foster argument as follows: 
For any $\mathrm{x}$,

(1) $\mathrm{x}$ is a physical object $\rightarrow \mathrm{x}$ is a space occupant

(2) $\mathrm{x}$ is a space occupant $\rightarrow \mathrm{x}$ is a substantial object

(3) $\mathrm{x}$ is a substantial object $\rightarrow \mathrm{x}$ has a non-power nature ergo, for any $\mathrm{x}$,

(4) $\mathrm{x}$ is a physical object $\rightarrow \mathrm{x}$ has a non-power nature.

Molnar makes the point that the word 'substantial' in premise (2) is problematic, since it is difficult to say without circularity what it means for a thing to be substantial. The common folk science view is that such things have bulk or volume (2003, p. 175). However, 'voluminous' refers to 'space-filling', not space-occupying, as Foster would have it. As noted earlier, Molnar argues that space-filling only applies to the macroworld whereas fundamental entities might be deemed space-occupying, and thus physically existent, without possessing fundamental qualitative properties.

A further problem with Foster's argument is the claim in premise (3) that if an object is substantial, then it has a non-power nature. Foster's argument has the burden of proof to show a necessary connection between being substantial and being nondispositional. Moreover, if having a non-power nature is attached to having a spatial address, Molnar notes that this is falsified by examples such as the point of maximum density in a vector force field, which exhibits no need for 'substance' (2003, p. 180).

In the special case of central forces for, say, gravitational effects, it is particularly easy to see that our ability to locate the source does not depend on any assumptions about the 'substantial character' of the source-object. The locus of a vector force (the point of maximum density in the relevant force field) unambiguously identifies the spatial point that the source of the force occupies (but does not 'fill'). The reasonable demand that moderate dispositionalism should deliver a spatial address for the source of the force can be met. The further demand that spatial location be identified with something more than occupancy is, in this case, unreasonable (2003, p. 180). 
Altogether, Swinburne's regress relies on assuming that primary properties, such as size and shape, can be directly perceived whereas pure-power properties can only be indirectly inferred from their effects. In the case of a pure-power world, it is argued, properties cannot be recognised because there is nothing detectable. Molnar argues, however, that fundamental qualitative properties are not detectable in the way Swinburne supposes, and that primary properties such as size and shape are macroworld features that tell us nothing about fundamental entities or properties. Moreover, Molnar shows why Foster's position - that pure-power worlds fail to specify power and therefore need bearers as recipients of the action of power-is based upon the assumption that non-dispositional properties are the only viable space-occupants. This assumption, Molnar argues, confuses space-occupying with space-filling, the former applying to fundamental entities but the latter, including primaries such as size and shape, being exclusive to macro-world entities. Thus Foster's argument does no work in making the case for the existence of fundamental qualitative properties.

\subsection{Extrinsic Properties}

Although Molnar defends Shoemaker against the requirement for fundamental categorical properties, he nonetheless is critical of the Causal Theory of Properties in terms of its causal criteria for properties to be genuine. For Shoemaker, all genuine properties are dispositional and intrinsic to their bearers. Moreover, they are identified by their respective causal contributions to the powers of their bearers (1980b, p. 294; 1984a, pp. 212-213; 1980). Accordingly, extrinsic relations between entities - for example, spatiotemporal and historical relations-are 'mere-Cambridge' and not causal contributors to the powers of property-bearers. Shoemaker considers these mere-Cambridge relations as being supervenient upon, and therefore nothing over and above, genuine properties and relations. The distance between a burning barn and an onlooker, for example, can be said to supervene upon all of the relevant ontologicallyrobust entities to which it applies. Since the distance is mere-Cambridge, and not causally relevant in and of itself, it is ontologically eliminable.

Molnar uses spatiotemporal relations as a counterexample to Shoemaker's criteria for genuine properties or relations (2003, p. 159): i) causally contributing; and ii) intrinsic to their bearers. He points out a conflict between the two criteria: 
a) By Shoemaker's criterion, all genuine properties and relations are powers and vice versa, since they causally contribute;

b) By Shoemaker's criterion, all genuine properties are intrinsic;

c) Spatiotemporal properties and relations do causally contribute, and by (i) are genuine properties;

d) Spatiotemporal properties and relations are not intrinsic, and by (ii) are not genuine properties.

Hence, either (i) or (ii) is not correct.

The arguments put forward by Swinburne, Martin and Foster are designed to show that (i) is incorrect. These propose that the identity of properties is not effectively given by the causal contribution that properties make, but instead, by their quiddity - by 'something else' over and above their causal role. The claim is that the identity of properties is given by the categorical, since this ultimately grounds dispositional properties. As shown in Chapter 13.1, Molnar argues against Swinburne's, Foster's and Martin's claims that perception of properties requires the existence of fundamental categorical properties. He contends that it is not clear how purely quiddistic properties could be detected. This, together with the fact that paradigmatic categorical properties such as size and shape seem restricted to macroworld entities, disallows asserting the existence of fundamental, categorical properties.

Molnar's course of action is to argue, instead, that (ii) is incorrect, that certain extrinsic properties and relations are genuine and thus ontologically ineliminable. If spatiotemporal relations, such as distance, can be shown to be causally contributing, then by this criterion they should be accepted as genuine properties, but nonetheless extrinsic. Molnar argues this by first attempting to establish that spatiotemporal properties are extrinsic; and second, by shoring up the claim that spatiotemporal relations are causally contributing.

\subsubsection{Argument 1: Molnar's Argument Against Foundationism}

Shoemaker's supervenient view of spatiotemporal relations resembles the Foundationist perspective adopted by Keith Campbell, whereby all spatiotemporal 
relations are accounted for by reference to the ontologically-robust foundations of the universe, which he considers to be the overlapping physicalised spacetime fields (Campbell, 1976, 1990; Molnar, 2003). By denying the existence of distinct objects, Foundationism ontologically eliminates extrinsic spatiotemporal relations. In arguing against Foundationism, Molnar defends the status of spatiotemporal relations as extrinsic and ontologically ineliminable. The issue raised by extrinsic relations and properties concerns what it is that instantiates them. It would seem that they must be instantiated by a system that encompasses the extrinsic relation as well as its relata, in which case the relation is apparently intrinsic to the instantiating system. Extending this idea results in Foundational-monism, presented by Campbell such that all the objects of the world are indefinitely extended, overlapping sub-regions of the spacetime field.

Foundationism regards relational facts, such as distances between objects, as supervenient upon their relata, and as nothing robustly over and above the 'foundational facts' (1990, p. 101). It differs from Relationism in that these foundational facts include both the relata and 'physicalised' spacetime. A Relationalist picture of spacetime, in which distance and orientation, for example, are nothing more than relational difference, by denying that spacetime is itself reducible to merely relations between objects (1990, p. 126). However, Campbell's position does not constitute a Substantivalist view, since his spacetime is not absolute and distinct from its contents. Instead, Campbell adopts a perspective in-between these two traditional stances: bodies correspond to overlapping sub-regions of the spacetime field and give rise to spacetime.

This in-between position should not be interpreted as an identity between spacetime and its contents, however. Campbell's physicalisation of space differs from John Wheeler's geometrization of spacetime - a theoretical approach that attempted to unify the forces of nature and was a forerunner to current research in quantum gravity (1962). Whereas the earlier Geometrodynamic Theory proposed to identify physical characteristics (mass/energy) and spacetime curvature, Campbell suggests that the physical characteristics of the universe produce spacetime curvature (1990, pp. 128129). The physical characteristics such as mass/energy are seen by Campbell as primary, and also categorical (1976). Applied to a field theoretical view of the 
universe in which physical reality consists of superimposable fields, bodies are appointed as sub-regions of the field. Since they produce the curvature of this spacetime field, and as position is given with respect to the field, bodies do not have their positions contingently. Campbell says that 'It is of their essence that they have the location they have and stand in the relations they stand in to all other sub-regions' (1990, pp. 129-130).

The theory does away with distinct objects, replacing them with sub-regions, described by Campbell as an 'orderly succession of fluctuations in the values of the fields in neighbouring sub-regions' (Campbell, 1990, p. 130). Manifest objects merely appear distinct from other bodies, and thus seem 'ontologically ineliminable'. Thus, what appears to obtain in terms of the 'manifest image' does not hold for the 'scientific image', which describes loci of power density regions giving rise to our higher order perception of objects. Campbell highlights the continuity of power density, with overlapping potential gradients such that fields emanating from dense loci are not truly distinct one from the other. By this reasoning, the position of any locus is related to all regions of the continuous, ontologically-robust field, without constituting extrinsic relations between distinct things.

Campbell's view is outlined in more detail in Section 12.1 of this Thesis, and I refer the reader to Molnar's 2003 book, Powers: A Study in Metaphysics, for more detail. In brief, Molnar summarises Campbell's fieldscape in three parts: i) superimposed and density-variable overlapping fields are the only 'occupants' of spacetime; ii) these variably-dense sub-regions correspond to the manifest image of bodies. The variable density is real, not merely epistemic, and these sub-regions are not physically distinct from each other or the fieldscape itself; and iii) each body, as an overlapping field, occupies the whole of spacetime; and does so essentially (2003, pp. 53-54). I read Molnar's analysis of Foundationism as an attempt to thwart the possibility of Campbell's reducible relations that serve as counterexamples to Molnar's claim for relations being extrinsic. I will argue, later in this section, that although Molnar's arguments against Foundationism are not successful, nonetheless his points are troublesome for the Causal Theory of Properties.

Molnar's rebuttal of Foundationism comprises three premises: i) 'all energy fields are discontinuous below the distance measured by Planck's constant', hence the 
world ultimately consists of discrete objects; ii) 'field strength, as measured at a point, varies over the entire extent of the field'; and iii) 'the strength of a point in the field, at a time, is not an essential, but a contingent, feature of the field' (since field density-ata-point is not necessarily constant) (Molnar, 2003, p. 53). Premise (i) suggests that the positions of loci, since discrete at levels below the Planck scale, vary independently. Premises (ii) and (iii) together suggest that locus position depends on the field strength at every point, and that, if these values contingently exist, so too does the locus position. Molnar concludes that position is an external, contingent relation with which we cannot dispense.

By way of response, I argue that premise (i) is contentious. Although manifestations of power may not occur below the order fixed by Planck's constant, Quantum Mechanics nevertheless requires field continuity, albeit that of a field whose manifestations are quantised. Whereas energy is absorbed and emitted only in Planck's quanta, the field itself need not be so stepwise in nature. Perhaps merely 'measurable' processes, as understood in terms of currently recognised laws, do not operate below the order of Planck's constant. The constant may thus enforce discreteness more in epistemic terms rather than ontic. In this light, the 'zero-point energy' of the vacuum could amount to an instrumentally defined minimum. Moreover, taking quantisation to imply fundamentally discrete entities could well be unwarranted. Recent work in quantum gravity attempts to explain the spacetime field in terms of more fundamental, but purely relational, processes. Loop Quantum Gravity (LQG), for example, puts forward overlapping fields comprised of excited field lines as fundamental (Smolin, 1991, 1997, 2000, 2006). This theory provides for emergent, stable conserved quantities and fermionic entities arising from a purely relational universe. Reminiscent of Campbell's overlapping fields, LQG proposes that spacetime derives from, rather than being constituted by, relational primitives. According to such an approach, contra the Swinburne regress and neo-regress arguments, discreteness or individuation of higher-order entities and powers, including that of spacetime itself, may obtain relationally, without primitive discontinuity. It is possible, therefore, that quantisation occurs without distinctness of entities at the most fundamental levels. 
The cogency of Molnar's premises (ii) and (iii) is similarly undermined by the points just made. Constraining himself to the Planck scale as a minimum of measurability, in referring to 'measured' field strength, Molnar is essentially drawing upon epistemological considerations, which are unarguably contingent. He ultimately begs the question by declaring that quantum indeterminacy equates to intrinsic contingency in the ontological status of fields. The understanding of how epistemology and ontology relate to quantum uncertainty remains subject to future scientific investigation.

If these provisional answers to Molnar's objections are acceptable, then a field-theoretic view, as provided by Campbell, is capable of supporting the contention that spatiotemporal relations may be neither extrinsic nor ontologically robust. Nevertheless, Shoemaker's Causal Theory of Properties is not a field-theoretic view; and unlike Foundationism, builds in discrete objects. The upshot is that Molnar's view that spatiotemporal properties are extrinsic only applies to the Causal Theory of Properties in virtue of its adoption of discrete objects. This point will be revisited in Chapter 13.3, in discussing Armstrong's criticism of Shoemaker's theory. I now turn to the second phase of Molnar's objection against Shoemaker's causal criteria for properties.

\subsubsection{Argument 2: Relational Properties, Although Not Powers, are Causally Relevant}

The second part of Molnar's approach backs up the claim that spatiotemporal relations are causally relevant although not intrinsic. Molnar puts forward his commonsense argument to show that powers and properties should be considered independent rather than reducible to each other as Shoemaker suggests. Changes in an object's properties, such as location in space, can occur without corresponding changes in its intrinsic powers. The S-property argument is then used to show that these spatiotemporal properties (and relations) are causally contributing, and should therefore be considered ontologically-robust, genuine properties. In this way, Molnar arrives at the conclusion that there are some causally contributing properties that are not intrinsic powers. In this section I argue that although the commonsense argument fails, the S-properties argument is sustained. 


\subsubsection{The Commonsense Argument}

Shoemaker's definition of a genuine property relies upon its causal efficacy. Molnar argues that powers, because intrinsic, are 'portable' such that an object can be spatiotemporally translated without changing any of its essential powers (Molnar, 2003, p. 159). He presents the examples of Socrates and a ballerina, Pavlova, changing their physical postures (standing, sitting, standing on tip-toes) and then returning to their original positions. Despite changing spatiotemporal properties, each ends the sequence with the same powers possessed at the beginning (2003, p. 159). Since there is a change in their properties but not in their intrinsic powers, there must be some properties, that are not powers. ${ }^{17}$

This argument rests on two premises: a) that there is no change in power associated with the spatial translation (as described in this specific scenario); and b) that spatial location is a property of an object. Regardless of whether an object's location in space and time should be considered a property, Molnar's argument relies upon (a), but it can be argued that this premise is unsustainable, since both Socrates and Pavlova gain memories of their experiences, and such information corresponds to power. But perhaps memory is not a basic, physical power, and so is not what Molnar had in mind. Although this point is outside the scope of this paper, a brief response is that memory is ultimately reducible to basic powers, although non-reductionists may demur.

Then does the commonsense argument work for non-sentient objects? Does a ball, if rolled to the left and back to the right, acquire any change in its intrinsic powers? Firstly, in the realm of common sense, macroscopic objects must age. This naturally modifies their powers, but spatial translation also entails motion which has effects over and above those of persistence. With reference to General Relativity, any round trip involves acceleration. If the ball moved quickly to the next galaxy and back, it would clearly be subject to time dilation. Let us say the ball is made of some rubber that 'ordinarily' crumbles after fifty years. Returning from a 'speedy' hundredyear journey, the ball may have aged only one year, so it would be far from

\footnotetext{
${ }^{17}$ We could possibly further explore the case whereby they change their spatiotemporal properties but don't return to their original position. In this case Socrates and Pavlova have changed both their spatiotemporal properties and arguably their powers. However, Molnar does not address that scenario in presenting this example.
} 
crumbling, indicating a clear change in its intrinsic powers as compared to those of a twin that had moved nowhere. This result generalises to a journey of even one metre.

What about something that does not age, like a proton? Does proximity to an electron, for example, amount to an extrinsic circumstance or some actual change in the proton's powers? Molnar relies upon a caveat that comparison of an object's powers is valid only between locations that are 'relevantly similar' such that 'locations per se are not empowering or disempowering' (2003, p. 160). This is essentially saying that we simply cannot count changes in circumstances which may indeed influence the parameters of how an object behaves. Since a change in spatial or temporal location arguably just is a change in circumstances, at least from a Relationist perspective, Molnar is thereby begging the question of what constitutes mere-Cambridge as opposed to bona fide change. (There is also the implicit assumption of ontological independence - that an object of interest is distinct from its surroundings - but this is allowed in the context of Shoemaker's theory). The commonsense argument therefore fails to show that spatiotemporal relations and powers are independent, because it does not succeed in demonstrating that a change in spatiotemporal properties may occur without a change in powers.

\subsubsection{S-Properties}

The S-property argument attempts to make a similar point to that of the commonsense argument. Its success lies in that it does so more precisely; but as I will argue in this Chapter, it only succeeds because Shoemaker proposes distinctness between objectbearers. (The argument itself is very similar to that put forward by Brian Ellis in the case of categorical dimensions, discussed in more detail in Chapters 6 and 7. There, I argue that Ellis cannot sustain an argument that the dimensions are categorical, since although perhaps passive rather than active in terms of causal contribution, they are nonetheless not independent of powers.)

The S-property argument shows that objects have intrinsic powers which are sensitive to location and distance, and in virtue of which they are, in turn, affected by location and distance. Taking his cue from Richard Feynman, R.B. Leighton, and M. Sands (Feynman et al., 1963, pp. 521-523; Molnar, 2003, p. 160), Molnar puts forward 'S-properties' as those involved in symmetry operations. A symmetry 
operation may be defined as an operation on a particular that at its conclusion leaves all but one of the salient physical properties of the particular unchanged (2003, p. 160). These S-properties, all of which are positional properties except for the interchange of identical particles, include translation in space, translation in time, rotation through a fixed angle, uniform velocity in a straight line, and reversal of time. While not being powers, S-properties moderate the forces among objects, because the powers of objects are fundamentally 'sensitive' to them. In this role, positional properties are causally relevant to every physical event (2003, p. 163).

Molnar argues that powers are fundamentally location-sensitive such that different positional circumstances of force-bearers will produce different outcomes of the manifestation of the very same forces. Formally this can be described as: ' $a$ ' $\mathrm{s}$ being at $p_{1}$ (at $t_{1}$ ) is not among $a$ 's powers, but it co-determines the strength of the forces that $a$ can exert on anything that is not also at $p_{1}$ at $t_{1}^{\prime}$ (2003, p. 165). Moreover, sensitivity is inherent since it derives from the nature of the powers that are manifested by their force-bearers (2003, pp.163-164). While location-sensitivity resides intrinsically within objects and is part of the essential nature of power, it is moderated by, being sensitive to, relational properties such as location or distance. Therefore, it would seem that distances between things do affect the outcomes of the operation of powers, although these distances are not themselves powers (2003, pp. 164-165). Spatiotemporal relations play a causal role in moderating the intrinsic powers of objects. Molnar defines this sense of 'intrinsic' in terms of an object possessing its powers independently of any other object (2003, pp.39, 102). The problem seems to be that relations extrinsic to the powers possessed by power-bearers can nonetheless affect these powers. Thus these extrinsic relations are causally potent.

Given that Shoemaker's spatiotemporal relations are mere-Cambridge properties, is it possible that the causal potency of these relations exist by virtue of being supervenient upon their relevant relata? In the case of a foundation-monist worldview where objects are not strictly distinct from one another, perhaps so. However, for a view that postulates distinct objects, I argue that such an answer falls short of explaining how intrinsic powers could operate on other distinct bodies without allowing for ontologically-robust, contingent relations between them. In this case, both relata of these contingent relations must be causally relevant. The operation 
of location sensitivity relies on the powers of multiple, distinct relata being moderated by all other co-relata. Letting $m_{1}$ and $m_{2}$ be two distinct masses, the force of gravity between them will be moderated by their separation according to Newton's Law of Gravity: $\mathrm{F}=\mathrm{Gm}_{1} \cdot \mathrm{m}_{2} / \mathrm{d}^{2}$. If spatiotemporal properties are supervenient, then $m_{1}$ and $m_{2}$ are both responsible for the causal role played by $d$. Although the sensitivity of $m_{l}$ and $m_{2}$ is supposedly intrinsic to these objects, nonetheless the sensitivity appears to implicate both objects.

A solution available to Shoemaker is to treat $m_{1}-m_{2}$ as a single system and hold distance, $d$, to be supervenient upon that complex, but finally singular, 'objectsystem'. In this way $d$ can be 'grounded in' $m_{1}-m_{2}$, and intrinsic to the instantiating system. However, since the $m_{1}-m_{2}$ system also stands in relation to other systems external to itself, the same difficulty and solution would apply, so as to combine these systems in much the same way that $m_{1}-m_{2}$ are unified. This leads to the unification of more and more complex systems, creating a regress that ends with a single system upon which the spatiotemporal relations between non-distinct parts of the system supervene.

This solution would work against Molnar's claim for spatiotemporal relations being causally contributing and extrinsic, since the end-result would correspond to a Foundation-Monism whereby spatiotemporal relations are intrinsic to the universe as a whole. This is the upshot of Foundationism, but unlike Shoemaker, Campbell does not allow ontologically distinct objects, from which the intrinsic/extrinsic divide of Shoemaker's world derives. The idea of properties being intrinsic, as a criterion for being genuine, seems meaningless without the comparative reality of extrinsic properties. The solution I have outlined for Shoemaker only works by repudiating the distinctness of objects in favour of some rendition of Foundation-Monism. So, without this step, even if Molnar does not succeed in refuting Foundationism, this does not save the Causal Theory of Properties, as set out by Shoemaker in the early 1980s, from Molnar's criticism of the criteria for genuine properties being both causally contributing and extrinsic.

In summary, this section has argued that Molnar's argument against Foundationism is contestable. Much of that argument relies on, as yet, unknown questions of physics. Therefore, Molnar has not successfully sustained the claim that 
relations cannot supervene in the case of field theories. Neither does his commonsense case support a denial of spatiotemporal relations as supervenient in a world of distinct objects. However, his S-property case does open the way to claim that spatiotemporal relations play a causal role in the moderation of intrinsic powers of their bearers. Attempts to side step this problem lead to a unification regress that can only end, ultimately, in a Foundational-monist theory. However, such a theory is very different from the world of distinct objects that Shoemaker proposes. The next section explores the consequence of combining a pure-power theory, such as the early Causal Theory of Properties, with an ontology that supports distinct objects. I argue that the two stances are incompatible.

\subsection{Pure-power and Distinct Objects}

Let us say that the manifest, macro-world objects are complex, mereological entities. For Shoemaker, complex objects are imbued with sets of conditional powers via the properties of each of their constituent parts. Thus the relations between parts of the complex object are involved in the existence of the overall powers of these objects. Extending the principle, the same applies to systems of complex objects that derive their power partly from relations between system parts. In what follows, I develop the case concerning systems. However, this case can also be generalised to complex objects, since they, themselves, can be thought of in terms of systems of more fundamental parts.

Shoemaker puts forward distinctness of particulars, yet advocates necessary laws of nature, i.e. necessary relations between properties. He means that laws specify or describe relations between properties intrinsic to the objects in which they are instantiated (1984a, pp. 222-223). A formal definition of 'intrinsic' is given by Molnar: ' $P$ is intrinsic to $x$ iff $x$ 's having $P$, and $x$ 's lacking $P$, are independent of the existence, and the non-existence, of any contingent object wholly distinct from $x$, (2003, p. 102). The Causal Theory of Properties posits a world whose objects appear to rely on extrinsic relations in order to build up complex objects and systems, and yet for Shoemaker the extrinsic relations are supervenient on their relata, the properties of which are construed as pure power. This situation poses at least three closely related, interdependent difficulties. i) It violates David Hume's Principle of Independence; 
ii) it underdetermines how circumstances for manifestation of power can be specified; and iii) it quells the ability to distinguish between a power's existence and its manifestation. These will be discussed in turn.

\subsubsection{The Principle of Independence}

Shoemaker's relations between one object and another are effectively $d$-relational (see the discussion on pp. 2-3 of this Thesis; (Francescotti, 1999)), since Shoemaker's objects are distinct from each other (or in Langton and Lewis's terminology, are relations between unaccompanied objects (Langton \& Lewis, 1998)). According to David Lewis, properties and relations which are entirely intrinsic are internal properties (1983a, p. 197). It follows that properties and relations that are not entirely intrinsic are in some degree external to their relata. Since Shoemaker's spatiotemporal relations are both $d$-relational and not internal to their relata-objects needing to be located with respect to other objects or frames of reference-they are extrinsic relations. However, since they are mere-Cambridge, and nothing over and above their relata, given the existence of the relata, the relations between them would be necessarily existent. To illustrate the difficulty further, take a complex object that is comprised of many constituent particulars, which are themselves constituted by more fundamental particulars and so on. Each layered particular possesses a set of conditional powers bestowed upon it by the properties it instantiates. The mereological combination of all of these particulars and their powers forms a complex object which itself possesses a cluster of conditional powers. If power is strictly intrinsic to its bearer, as Shoemaker suggests, then the set of overall conditional powers possessed by the complex object involves extrinsic relations between each of the constitutive particulars. If these extrinsic relations are, themselves mereCambridge and thus nothing over and above their respective sets of constitutive particulars, then these would appear to be necessarily existent. Given the existence of the parts and their properties of a complex object, the relations between the parts cannot fail to obtain. Thus, it would seem that there must be something in the properties of objects and their constitutive parts that governs how they relate to one another. In Shoemaker's schema, the constituents of a molecule of water (two hydrogen atoms and an oxygen atom), for example, are bound together by relations 
that supervene upon the respective atoms. The 'glue' of binding is provided by the properties of each of the atoms themselves. Thus it would appear, given that all properties are ultimately sets of causal potentialities and powers, that the relations between the atoms are necessary. However, because the atoms are distinct entities in this schema, the relations between them seem to be extrinsic. So, pure-power theories with distinct objects apparently entail extrinsic but necessary relations between distinct particulars.

Armstrong points out an incompatibility between relations that are both necessary and extrinsic, observing that this represents a situation ruled out by the Principle of Independence, whereby 'there are no necessary connections between (wholly) distinct existences' (Armstrong, 2000, p. 8). For Armstrong, necessary connections may occur only between entities that are not distinct; and in a world of distinct objects, relations between them must be self-contained, contingently exist and be categorical. This objection can be applied equally to systems of complex objects. Rejecting the Principle of Independence, by adopting necessary but extrinsic relations, demands an account of how extrinsic relations might be necessitated. Shoemaker argues that necessity is provided in virtue of extrinsic relations being grounded in the (pure-power) properties of their relata. However, in the case of mere-Cambridge relations supposedly tying the relata together, there is actually nothing over and above the relata themselves, which therefore cannot be strictly extrinsic.

It is evident that the terms 'extrinsic' and 'intrinsic' represent relative notions. Relations between particulars which together comprise complex object $a$ are 'extrinsic' to those particulars, yet intrinsic to object $a$ itself. Likewise, relations between complex objects $a$ and $b$ are extrinsic to $a$ and $b$, but intrinsic to an $a-b$ system. Even if the terms 'extrinsic' and 'intrinsic' are fully relationally specified, their appearance, in general, is derived in virtue of the relata in question being distinct. The core of the problem remains, that relations between distinct objects seem to require that these relations be self-contained, and thus are categorical and contingently exist. 


\subsubsection{Specifying Circumstances for Power Manifestation}

For Shoemaker, laws are necessary-the laws simply spell out the causal potentialities of properties and relations that exist in the world: given what exists in the world, we have the laws. The properties — sets of primitive causal potentialities and relations between them give rise to the conditional powers of the things that instantiate them. These properties (and relations) are intrinsic to the objects that instantiate them. Recall the definition of intrinsic given on pages 2-4 of this Thesis, summed up nicely by George Molnar's definition: 'intrinsic properties are those the having of which by an object in no way depends on what other objects exist' (2003, p. 39). Stated by him more formally, ' $P$ is intrinsic to $x$ iff $x$ 's having $P$, and $x$ 's lacking $\mathrm{P}$, are independent of the existence, and the non-existence, of any contingent object wholly distinct from x' (p. 102). This, as Molnar notes, makes 'intrinsic' a modal concept (p. 39). If a thing's properties and relations are intrinsic, genuine properties of the objects instantiating them, then they are independent of anything external to the object.

For Shoemaker, although the cluster of conditional powers possessed by objects are intrinsic to the object, the picture we are given is of manifestation of powers that can only occur due to the extrinsic circumstances in which the object exists. Notice the following, 'A different distinction is between powers, in a sense I am about to explain, and the properties in virtue of which things have the power they have' (Shoemaker, 1980a, p. 211). By 'in virtue', as Shoemaker explains in the accompanying footnote, he means 'it is a lawlike truth that whatever has those properties has that power'. He continues on to make the very important point that, 'For something to have a power, in this sense, is for it to be such that its presence in circumstances of a particular sort will have certain effects. One can think of such a power as a function from circumstances to effects' (1980a, p. 211). Again, he uses a footnote to clarify what he means when he talks about 'circumstances':

In speaking of "circumstances" I have in mind the relation of the object to other objects; instead of speaking of "presence in circumstances of a particular sort" I could instead speak of "possession of particular relational properties". 
Being in such and such circumstances is a mere-Cambridge property of an object, not a genuine (intrinsic) property of it (1984a, p. 211, n).

So, on one hand, Shoemaker has conditional powers arising from properties and the relations between those properties; and these conditional powers are intrinsic to objects. By the above definition, these conditional powers exist independently of any external object; and external objects are wholly distinct from one another. But, on the other hand, he seems to require some kind of 'circumstance' in order for an object's conditional powers to manifest as power; and the 'circumstance' in question, we are told, is precisely the relation of one object to other objects. Power, furthermore, is a function from the state of an object to other objects - to effects.

While powers are intrinsic to their bearers, how they manifest-that is, the particular way they affect other objects - depends on the relations between the powerbearer and those objects. For Shoemaker, such circumstances do not endow an object with power; this task belongs to the properties instantiated by the object. Once so endowed, however, an object will manifest its power differentially according to circumstances. In this respect, Shoemaker's description of power takes the form as a 'function', which can be understood as an operational relation, from circumstances to effects (1984a, p. 211, n). David Armstrong makes this interpretation of Shoemaker's ontology clear, when he writes:

Suppose that the world consists of particulars having properties in the narrow sense and related to each other by external relations. (Internal relations may, I trust, be ignored as not constituting any ontological addition. The truthmakers for such relations are just the terms of the relation.) Now suppose that these properties and relations are nothing but powers. It will follow that the manifestations of these powers, when they occur, can themselves be nothing but cases of particulars coming to have certain powers. After all, manifestations are nothing but certain particulars coming to have certain properties, and on the theory being criticized all properties dissolve into powers. But could there be a world of this sort? Powers must surely issue in manifestations that are something more than just powers. A world where potency never issued in act, 
but only in more potency would be one where one travelled without ever having the possibility of arriving (Armstrong, 2004b, pp. 138-139).

Teasing out the role of power in this description, it determines how extrinsic relations between objects occur such that certain effects obtain. If the extrinsic relations between objects were contingent, a power could accordingly produce various manifestations-effects, depending on the natures of the contingent relations in question. However, since these relations are supervenient upon the genuine properties of the objects that they take to be their relata, and thereby supervenient on pure-power properties, it would seem, as Alexander Bird notes, that these relations between objects are built-in, being given in terms of the powers of the objects themselves (2005c, pp. 535-536). However, specifying such relations for each manifestation of power involves an indefinite number of relations. Two hydrogen atoms and an oxygen atom, for example, may form a water molecule depending on the relations between them. Since these relations are supervenient on each of the respective atoms, it must be in virtue of the powers of each atom that the water molecule forms. Yet it will only do so, and persist, within specific ranges of temperature and pressure, thereby involving further atoms and their motions. These, in turn, have relations with other atoms and so on. A complete specification of the circumstances for forming one molecule involves, albeit indirectly, every object in the universe. Therefore the totality of the universe is required to fully explain each relation between its objectcontents.

On the face of it, since this very same totality explains every other such relation, any particular relation seems underdetermined. Perhaps a solution can be given in terms of the arrangement of the totality, providing Shoemaker with a unique specification for every manifestation relation? But such an arrangement would depend on relations between parts, and these relations, being supervenient, must be given in terms of the totality of objects in the universe.

\subsubsection{Existence Versus Manifestation of Power}

Applying the constraint that the extrinsic relations are supervenient upon the powers, which are identified by their effects, leads to a situation whereby no wedge can 
feasibly be imposed between power and its manifestation. Power can be defined as the ability that objects have (in virtue of their properties) to affect other objects or be affected by them. The identity of properties with power and the resulting intrinsicality of power indicates that it is borne independently of relations that its bearer may have to other objects. However, given Shoemaker's definition of 'circumstance', a power's manifestation requires that at least one relation exist between the bearer and another object. Taking a universe that has only a single entity (and discounting frames of reference as constituting plausible relata), the power possessed by that entity could never manifest, since there would be no second-place relata available. Although unmanifested powers are not for this paper to address generally, such thought experiments raise the question of whether powers can be defined in terms of the effects they allow their bearers to exert, where such effects are not possible.

It can be argued that certain repercussions arising from the single-object world apply directly to Shoemaker's situation, given that his is a pure-power theory. Shoemaker describes power as a function from the relational states of an object to its effects. These relational states are the relevant circumstances, representing the relations between the object and others. This can be set out more formally: let $f$ (for function) be a certain power of an object of interest, say $a$. And let the 'circumstances' be a relation R between $a$ and 'another relevant object or set of objects' $b$. Then $a$ 's power is expressed as: $f(\mathrm{R} a b) \rightarrow$ effects. But the effects in question are precisely the manifestation of $a$ 's power, so we could equally write: $f(\mathrm{R} a b) \rightarrow$ manifestation of $f$.

While it is clear that $f$ needs $\mathrm{R} a b$ in order to be manifested, it does not necessarily follow that $R$ is causally relevant, i.e. makes a causal contribution to the outcome, since the crucial causal influence could as well be provided by the intrinsic properties of $a$ and $b$. I believe this is the scenario that Shoemaker puts forward in his account of spatiotemporal relations being mere-Cambridge. However, even if $\mathrm{R}$ is not causally relevant, it is supervenient upon $a$ and $b$. In this case, $f$ needs at least $a$ and $b$ to exist in order to be manifested. While this does show that some particular external object is part of $a$ 's ability to manifest any particular power from its set of conditional powers in any particular way, it does not show that the intrinsic properties of $b$ itself are necessarily part of $a$ 's ability to manifest power per se, since $a$ 's cluster of conditional powers may represent the ability to manifest in various other ways, given 
different relations with other combinations of external objects. Although $a$ does not require $b$, in particular, for the manifestation of its powers, $a$ does require some external object—-some variable entity $b^{\prime}$ —in general, in order to manifest its power in some way.

I conclude from the above that, for Shoemaker, the existence of objects external to $a$ is necessary to provide part of $a$ 's ability to manifest power, although the manner of such manifestation will be contingent upon what external objects are involved. We thus observe that $a$ 's power is intrinsic and therefore independent of anything external to $a$; yet for $a$ to manifest its power in some way, at least one external object is required. The catch is that for the Causal Theory of Properties, power by its nature is precisely the ability it bestows upon its bearer to affect or be affected, and these effects are the manifestation of power. Yet, in no way do extrinsic relations, or circumstances, drive a wedge between power and its manifestation if those extrinsic relations are nothing over and above the powers themselves. Theorists advocating categorical properties may possibly fall back on these to make a distinction between power-existence and its power-manifestation, but the Causal Theory of Properties must do without, for power and its manifestation are interdependent. Since powers reduce to properties and properties to powers, and both reduce to effects, for a pure-power theory such as that presented by early Shoemaker, power that cannot manifest does not exist. Moreover, if the manifestation of power requires the existence of at least one object apart from that of its bearer, and if power cannot exist without possibility of manifestation, then power cannot be entirely intrinsic.

In order to redress the reduction underlying this violation of the Principle of Independence, and problems involved in the specification and manifestation of power, Shoemaker puts forward a later revision of the Causal Theory of Properties, which is discussed in the next section.

\subsection{Revisions}

The early Causal Theory of Properties purports that each genuine property is a set of causal potentialities, each set being identified with a property (Shoemaker, 1980b, pp. 294-296; Shoemaker, 1984a, pp. 217-221). Such properties contribute to the 
conditional powers of their instantiating objects by forming clusters of conditional powers (Shoemaker, 1984a, p. 213). Each cluster of conditional powers is also identified by Shoemaker as a property:

When a thing has a power conditionally upon the possession of certain properties, let us say that this amounts to its having a conditional power...Having introduced this notion of a conditional power, we can express my view by saying that properties are clusters of conditional powers...And the causal potentialities that are essential to a property correspond to the conditional powers that make up the cluster with which the property can be identified (Shoemaker, 1984a, p. 213).

This formulation represents the difficulty that properties can be identified both as parts of clusters of conditional powers, and also as clusters themselves. I think that these two different ways of identifying properties can be interpreted in early Shoemaker in terms of differing levels of properties. The prospect of properties being reducible to powers, and vice versa, becomes the target of Shoemaker's 1998 reforms. In this later formulation, Shoemaker: i) posits properties as possessing essential forward- and backward-looking causal features, rather than being comprised of them (1998, p. 64); and ii) revokes the claim that properties are conditional powers (1998, p. 63). I will discuss the implications in turn.

\subsubsection{Properties Possessing Essential Causal Features}

Shoemaker's move to allow properties to possess essential causal features, rather than being comprised of causal potentialities, introduces contingency into the theory. His changes also allow the possibility of categorical properties, if by this we mean properties that make no causal contribution to the powers of objects; although he is non-committal about what role they would play (1998, p. 65). His claim that properties have causal potentialities 'non-derivatively' (1998, p. 65) implies that potentialities are not further reducible. Other introductions in the 1998 revisions include allowing two or more properties to share all of their causal features, and yet remain distinct. Shoemaker allows merely 'being property $\mathrm{X}$ ' and 'being property $\mathrm{Y}$ ' 
to define different properties, indicating that there is something to properties $\mathrm{X}$ and $\mathrm{Y}$ that contributes to their identity over and above what they do; namely, what they are (1998, p. 64). These revisions suggest that there is more to properties than just their causal features, aligning the theory with calls by Swinburne et al. for there to be more to properties than powers (Armstrong, 1997, p. 80; Armstrong, 2000, pp. 13-14; Armstrong, 2004b, pp. 138-139; Ellis, 2001b, 2002, 2005b, 2008a; Foster, 1982, pp. 66-72; Heil, 2003a, pp. 76, 99-107; 2006, p. 42; Martin, 1993, 1996a, 1996b, 1996c). It also admits Heil's claim that qualitative properties are required for powers to be individuated or differentiated (2007, p. 84). Exactly what more there is to a property, aside from its essential features, is not explicated, although the fact of properties possessing rather than being comprised of their essential features seems to build in 'quiddity', described by Robert Black as whatever there is to a property over and above the power that it bestows on its bearer (2000). Importantly, this revision removes Shoemaker from what Armstrong dubs a 'Power-Maximalist' view (2004b, p. 139) or pure-power position to a much weaker stance that portrays causal features as merely essential to properties rather than comprising them.

\subsubsection{Properties No longer Clusters of Conditional Power}

The second revision revokes the identity of properties as clusters of conditional powers. Since the explanation of conditional power is given in terms of properties, these cannot circularly be explained in terms of powers $(1998$, p. 64). This suggests that conditional powers are no longer constitutive of their properties, but instead, supervene upon properties. This change has much in common with evolution of Categoricalism from a reductionist to a supervenient account of dispositions, although some important differences between the Categoricalist and Strong DE accounts emerge with respect to the laws of nature. As discussed in Chapters 2 and 3, Armstrong moved away from a reductionist stance, that identified dispositional properties with their categorical bases, toward viewing dispositions as occupying a causal role conditioned upon the laws of nature. Nevertheless, both the subvening microstructure and the laws were considered categorical. In contrast, Shoemaker (1998) posits conditional powers that are supervenient upon a base of dispositional, rather than categorical, properties. Unlike Armstrong's categorical laws that are 
explicitly included as partial truthmakers for dispositions, Shoemaker's laws are necessary, and derive automatically from the dispositional properties.

Usually the difference between supervenience and reduction is understood in terms of one-way versus two-way entailment, respectively. If the causal powers are supervenient upon an object's properties, then there is a correspondence between properties and powers, although not one-to-one, such that the properties 'fix' the powers but not vice versa. This creates the situation whereby two objects with the same powers can plausibly have two differing sets of properties. Thus, Shoemaker's later formulation no longer allows a cluster of conditional powers to be uniquely identified with any set of given properties. Previously we could only know about properties via the effects of the powers, and even that was a problematic affair. By moving to a supervenience position, Shoemaker now distinguishes a bearer's powers from its properties; an object may have various powers, yet not possess any specific properties that reliably correspond to those powers.

If properties can no longer be identified in terms of their contributions to power, then their existence appears to require that they achieve their identity by means other than causal roles. While Armstrong's supervenience of dispositions also encounters this challenge, he answers it by allowing the causal role of dispositions to be modified according to the contingent laws of nature. However, this solution is not open to Shoemaker's view in virtue of his necessary laws. Just as quiddity is introduced in Shoemaker's later modification, by allowing properties to have an identity in virtue of something other than their causal features (discussed in Chapter 13.4.1), so this step of supervening powers on properties presupposes something more to properties than those powers. As already noted, the introduction of quiddity turns Shoemaker's view from a pure-power theory into some sort of dualist or dual-aspect theory. In Section 3, although I do not re-visit the implications of Shoemaker's 1998 change specifically, I examine certain difficulties with views that allow properties or aspects of properties to be categorical or qualitative at the fundamental level.

\subsection{Summary and Conclusions}

In this chapter, I have discussed arguments against Shoemaker's Causal Theory of Properties, such as the Swinburne regress and Molnar's commonsense and S-property 
arguments. The Swinburne regress and neo-Swinburne arguments rely upon the assumption that fundamental categorical properties afford their own direct perception. Molnar argues that this assumption is unsustainable. His criticism of Shoemaker, instead, focuses on the causal criteria for genuine properties to be both causally contributing and intrinsic. Using spatiotemporal relations as a counterexample to Shoemaker's formulation of genuine properties, Molnar develops arguments from commonsense and S-properties to show that there is a contradiction between the two criteria. I have argued that while the argument from commonsense is flawed, Sproperties account opens the way to claim a causal role for spatiotemporal properties in moderating the intrinsic powers of their bearers for those theories that hold with distinct objects. This raises questions concerning the Principle of Independence; how circumstances for the manifestation of power are specified, and whether the existence of power is independent of its manifestation.

According to the Principle of Independence, for theories that postulate distinct objects, relations between such objects must be contingent, hence self-contained and categorical. Yet, the Causal Theory of Properties requires these relations to be supervenient upon pure-power properties. Thus, the categoricity required for the existence of distinct objects is missing in the Causal Theory of Properties as formulated in the early 1980s. This chapter further argued that in order to completely specify circumstances for the manifestation of power, every object in the universe is implicated. The result is that the totality of the universe is required to explain each and every relation between its object-contents, resulting in an underdetermination of local, extrinsic relations. The third problem concerned the inability of the Causal Theory of Properties to properly tease out power from its manifestation. In theories which posit fundamental categorical properties, the distinction between the existence and manifestation of power is formulated accordingly. Given that power is defined in terms of effect, and that the identity of properties resides in their causal contribution, Shoemaker's properties are reducible to powers. If the identity of powers resides in both effects and properties, then it appears that power cannot be entirely intrinsic; and how properties get to be individuated is problematic.

Redressing the difficulties incurred by reduction of powers to properties, and vice versa, requires that Shoemaker reformulate his theory in terms of either removing 
the reduction between powers and properties or crafting a Foundation-Monism, whereby the universe emerges from a single fundamental entity. The former option is his chosen path, presented in the later revision to the Causal Theory of Properties. I have argued that this course removes the theory from a pure-power view in favour of incorporating quiddity and hence fundamental categoricity. The alternative reformulation, postulating a fundamental monism and denying distinct objects, is the approach that Rom Harrè and other monist field-theorists adopt. Discussion of this perspective is deferred until Section 4 of this Thesis. 



\section{SECTION 3: \\ Property Compatibilism}

Sections 1 and 2 examined two different types of monist property theories: Categoricalism and the Causal Theory of Properties. Categoricalism faces the challenge of providing a satisfactory explanation for the necessity that underpins strong causation, without drawing on irreducible dispositionality to achieve this. Shoemaker's Causal Theory of Properties faces just the opposite problem, namely, that it lacks the categoricity required for the distinctness of objects and for discriminating between the existence and manifestation of power. To alleviate these property-monist concerns, some theorists propose that both categorical and dispositional properties exist with ontological robustness. The term 'Property Compatibilism' is given to such accounts, since they allege that fundamental dispositionality is compatibly accommodated with fundamental categoricity in some way. This section outlines and discusses the issues and concerns that Property Compatibilist theories face in attempting to explain how two different natures fit together at the fundamental level. 



\section{CHAPTER 6}

\section{BRIAN ELLIS:}

\section{NEW ESSENTIALISM - OUTLINE}

Brian Ellis (1999, 2001b, 2002, 2005a) envisions a highly structured world based on a hierarchy of physical natural kinds ${ }^{18}$, each of whose members are identical to those of their own kind, and different to those of every other kind, with respect to their intrinsic properties $(2001 \mathrm{~b}$, p. 31). Examples of natural kinds include different chemical elements (2001b, p. 3). An intrinsic property is defined by Ellis to be those properties possessed by every member of the kind that would be displayed in the absence of any accidental forces (2001b, p. 29). Those intrinsic properties that things have necessarily-such that they are the kinds of things that they are-comprise essential properties (2002, p. 54).

Ellis's Substance-Attribute view of a world describes three categories of natural kinds: substantive (substances and objects), dynamic (events and processes) and property (properties and structures) kinds (2001b, p. 74). These are subdivided, each into a hierarchy of levels of generality-global, species and infimic (from the term 'infima', referring to the least general level of taxonomic categorisation such that all entities of a kind at this level are identical with respect to their essential properties). The global level of substantive natural kinds is maximally determinate, including every kind of object or substance in the world (e.g. elements, compounds and fundamental particles). The global level of dynamic natural kinds includes all other kinds of events or processes that exist (e.g. causal process, causal interactions and energy transfer processes). The global level of natural property kinds includes all the kinds of properties and structures in the world (e.g. dispositional and categorical properties; spatial and temporal relations). Each of these global kinds is determinate for more specific kinds (or species) falling under each of their categories (e.g. substantive kinds: sodium salts, leptons; dynamic kinds: chemical reactions,

\footnotetext{
${ }^{18}$ Natural kinds are kinds of things of a material nature. New Essentialism posits natural kinds in a far more narrow way than Aristotle, who incorporated biological kinds. Ellis cites examples of natural kinds including basic physical and chemical substances such as species of molecules, subatomic particles and so forth.
} 
electromagnetic radiations; property kinds: mass, charge, field strength, shape, spatiotemporal interval). Each of the species-level kinds, in turn, includes the maximally determinable infimic levels of category kinds (e.g. substantive kinds: helium atoms, neutrons; dynamic kinds: $\mathrm{H}_{2}+\mathrm{Cl}_{2}=>2 \mathrm{HCl}$; property kinds: unit field strength, spherical shape). Each of the natural kinds is associated with their respective universals - substantive, dynamic and property universals; and the instances of the infimic level of each natural kind instantiate their respective universals. These property instances correspond to the 'classical' universals (2001b, p. 74).

Any natural kind is objectively "discrete" and "discontinuous" with respect to all other natural kinds. The discreteness is: i) built-in to the universe and is thus objectively present in nature; ii) derived from the intrinsic essential natures and structures of things rather than as an artefact of some arbitrary classificatory system (2001b, 19, p. 31); and iii) generated from the fundamental discreteness of quantum reality (2001b, p. 2). Ellis's universals are consistent with his picture of an objective, structured hierarchy. Ellis emphasises the ontologically-robust nature of the kinds, which are as much part of the furniture of the world as the instances that fall under each of these kinds. Hence, the kinds are not mere epistemic categorisations of the instances (2005b). We have epistemic access to the kinds because their instances exemplify the kinds themselves, and thus the processes of the same natural kind, including causal powers, operate in structurally similar ways.

\subsection{Properties and Dimensions}

Traditionally, dispositional and categorical properties have been put forward in attempts to describe the manifest world. They have often, although not always, been defined in mutually exclusive and somewhat oppositional terms (Ellis, 2001b, 2002). AS noted in Chapter 1 of this Thesis, Ellis writes that categorical properties have been considered readily imaginable (Ellis, 2002, p. 68); existing independently of behaviour (pp. 68-69); multi-dimensional (p. 69); structural (pp. 69-70); nondispositional or non-modal (pp. 70, 117); and grounding or realising of the dispositional (pp. 174-175). Adopting the concept of quiddity, described by Alexander Bird and Robert Black as referring to some 'nature' of properties independent of their causal roles (Bird, 2006; Black, 2000; Ellis, 2008a), Ellis asserts that categorical 
properties are quiddistic in the sense that they have their identity by virtue of what they are rather than by what they do (Ellis, forthcoming). Categorical properties have by and large been characterised in terms of spatially-extended or space-occupying properties represented by Lockean primaries of size, shape, solidity and so on (Locke, 1924, II, Ch. VIII, 8, 66). Strong Categoricalists, such as Armstrong, hold that all properties, including those at the fundamental level, are categorical.

Importantly for Ellis, categorical properties are structural properties, and he relies on these to underpin his natural-kinds hierarchy and its central tenet- that ontologically-robust structure is built into the universe (Ellis, 2001a, p. 174; 2001b, p. $2 ; 2002$, p. 68; 2005a, p. 382; 2008a). This dependency on structure requires it to exist at fundamental levels and to include spatiotemporal relations, as he considers space and time to be 'the pure forms of physical structure' (Ellis, 2002, p. 174). To accommodate current theories of physics, Ellis leaves open the idea that quantum fields might be fundamental quiddities, replacing the Lockean quiddities of Newtonian mechanics (forthcoming). Yet, as he observes, neither structure as relations between parts (Ellis, 2001b, p. 10; 2008b, p. 143), nor objects themselves (forthcoming), exist at fundamental levels.

As also noted in Chapter 1, dispositional properties have been contrasted with categorical properties in all of the descriptive contexts above. As Ellis points out, dispositional properties have been considered uni-dimensional (2002, p. 69); nonstructural (pp. 69-70); essentially modal (p. 70); and grounds for the categorical (pp. 174-175). Ellis defines dispositional properties as those that obtain their identity by virtue of what they dispose their bearers to $d o$ (forthcoming), rather than by what they are. Dispositional properties are essential properties, and are therefore all intrinsic to their bearers (2001b, p. 26; 2002, p. 54). The term 'intrinsic' is used by Ellis here to specifically refer to 'causal intrinsicality', which he defines as 'a property of the relation between a property and its bearer' (2001b, p. 27), and possessed by an object in the absence of accidental forces (2001b, p. 29). The causally intrinsic properties of things explain how those things behave, or are disposed to behave, in virtue of the intrinsic causal powers of their constituents (2001b, p. 31). 
Dualist positions, such as the New Essentialism advocated by Ellis ${ }^{19}$ (2001b, 2002, 2008b, forthcoming), hold that dispositional and categorical properties present a real difference in category between dispositional and categorical properties (forthcoming), whose mutual exclusion is based upon whether a property is structural or not (2002, p. 70).

Because categorical properties are structural, they are almost all considered to be what Ellis terms 'dimensions'. He describes dimensions as the quantitative properties that are involved in the laws of nature (forthcoming), which direct how the effects of causal power are distributed (Ellis, 2001a, 2008a). The effect of a causal process is to change the values of certain dimensions, and Ellis describes these dimensions as 'respects in which things may be the same or different' (2008a). They 'determine the structural frameworks within which the powers operate' (Ellis, 2001a, p. 174) and include, for example, quantities, shapes, duration, direction, spatiotemporal separation, position and time (Ellis, 2001b, pp. 136-138; 2008a). While the dimensions include most, if not all, of the categorical properties, they also include certain causal powers, since causal powers and capacities, like categorical dimensions, also represent 'respects in which things can be the same or different' (Ellis, 2008a). I refer to the latter as 'powerful dimensions'.

For Ellis, both dispositional and categorical properties play causal roles (2001b, pp. 9-10; 2005b, p. 470). ${ }^{20}$ The causal powers are 'active' forces, determining the behaviour of the objects that bear them. In contrast, categorical properties-such as sizes, shapes and spatiotemporal relations and locations - obtain identity in virtue of what they are rather than what they $d o$. Their causal role is passive, being that of merely 'factors' (forthcoming), rather than of driving forces, in the operation of causal processes. The contingency or necessity of a property's manifestation is of importance to this passive-active differentiation. With respect to categorical properties, even in relevantly appropriate circumstances, their manifestation is contingent. For Ellis, categorical properties are those that do not 'confer of necessity any power or

\footnotetext{
${ }^{19}$ First posited jointly with Caroline Lierse (Ellis \& Lierse, 1994).

${ }^{20}$ Causal powers are properties involved in physical causal processes and energy transmission. Capacities and potentials are dispositional properties, but do not necessarily involve transmission of energy (Ellis, 2008a).
} 
disposition' and do not essentially require manifestation in any distinctive fashion in response to an appropriate stimulus (Bird, 2005a, p. 439; Ellis, 2005b, p. 470).

In a forthcoming paper, Ellis describes the distinction between dispositional and categorical properties at the object level. In earlier writing, Ellis claims his categorical dimensions to be higher-order properties of causal powers (2002, p. 69; 2005 b, p. 470). Existing independently of how a bearer of a property is disposed to behave, they nonetheless were purported to play a causal role in 'determining' or 'directing' the behaviour in particular ways. Hence, they supply the circumstances for the behaviour:

These dimensions of the causal powers are the properties that I call categorical. They are real, and no less important in the overall scheme of things than the causal powers that have them essentially. In reality, they are second order properties - properties of properties. They are, indeed, amongst the essential properties of the causal powers (2005b, p. 470).

These higher-order structural properties were described as including shape, size and aggregation (2001b, p. 135). Characterising the categorical dimensions as higherorder properties of the causal powers turned out to be problematic, given that Ellis also referred to them as fundamental $(2001$ b, p. 218; 2002, pp. 70, 174) and in some passages 'more fundamental' than the causal powers (2001b, p. 138; 2005b, p. 470). Their fundamentality status is clarified in personal communication in which Ellis says that he no longer considers the categorical dimensions to be higher-order-properties of causal powers - since dimensions are 'presupposed' by the causal powers, and so must be fundamental (2008a). Further clarification is provided in a forthcoming paper in which Ellis describes the dimensions as 'determinables' (e.g. mass), each with at least two possible values or 'determinates' (e.g. $5 \mathrm{~kg}$ of mass), one of which is actual; and necessarily the dimensions are more ontologically fundamental than their values - mass must exist before one can have five kilograms of it. (Importantly, the dimensions are not constituted by their values. Mass, for example, exists as something over and above the fact of being quantifiable.) While Ellis does note that dimensions are 'among the fundamental constituents of reality at the object level' (forthcoming), 
rather than denoting dimensions as being actually fundamental, his specifying the 'object level' here leads me to interpret him as meaning that dimensions are actually present in the world in the sense of being ontologically-robust; his dimensions exist over and above their respectively many instantiated values.

One reason for talking about the difference between dispositional and categorical properties 'at the object-level', is because it is only at this level that talk of 'physical objects that are the bearers of causal powers' (forthcoming), and hence of spatiotemporal relations between objects, has meaning. The relations between mereological parts of complex objects also fit into this level of description. Consequently, structure is portrayed by Ellis in two different ways: first, as higherorder block structure (2001b, pp. 10, 247); and second, as the kind of quiddities, regardless of whether Lockean or quantum mechanical, that feature as fundamental categorical properties $(2001 \mathrm{~b}$, pp. 138, 218; 2002, p. 70). What stands out in the course of the above clarification is that categorical dimensions and causal powers are irreducible to each other, regardless of the level of reality under discussion; they represent different types of properties (2001b, pp. 4, 47, 49, 111, 217; 2005b, pp. 470471 ; forthcoming) that are purportedly different, ontologically-robust ways that reality can be divided (Mumford, 1998, p. 95).

In recent writing, Ellis describes the possibility of viewing dispositional properties in two different ways: Unconditional (e.g. 'propensities') and conditional (e.g. causal powers) (forthcoming). For Ellis, propensities are more primitive than causal powers, and their laws of action (discussed shortly) are independent of contingent circumstances that involve categorical properties. An example might be the propensity for a substance to undergo spontaneous radioactive decay. In contrast, conditional dispositional properties, such as causal powers, rely upon categorical properties such as location and spatiotemporal relations to provide the circumstances in which their relevant laws of action and reaction operate.

\subsection{Laws}

The laws of nature are not ontologically-robust properties or relations that exist independently (2001b, p. 128), but rather, are descriptions of how things are disposed to behave or interact in particular kinds of circumstances, by virtue of their causal 
powers (2001b, p. 206); for example, how the negative charges of electrons will behave in particular circumstances. The laws describe behavioural dispositions in terms of the essential natures of the kinds of things in the world (Ellis, 2002, pp. 59, 88,101 ), and are thus metaphysically necessary (Ellis, 2001b, p. 116; 2002, pp. 88, 100-101, 110-111). Ellis says that change occurs via laws of action and reaction, defined as how the dimensional values of an agent change or are changed by respective causal powers $(2001 b$, p. 206; 2008a). Laws are not 'accidental generalizations', albeit statistical laws describe probabilities (2002, p. 116).

For Ellis, the laws are structured in parallel with the hierarchy of natural kinds (2001b, pp. 4, 205-206), such that there are general, global laws that apply to all events or processes, e.g. conservation laws; and other laws that apply to general structures, e.g. spacetime or energy fields (2001b, p. 205). There are also laws concerning the essential natures of species level kinds, e.g. all electrons have a negative charge; as well as causal or statistical laws operating at the infimic level.

A difference between Categoricalism and New Essentialism can be noted with respect to the contingency of laws (Ellis, 2002, pp.59-63). The laws of the mechanistic or passivist paradigms, such as Categoricalism, are contingent and prescribe how inert, categorical, self-contained properties relate (2001b, p. 206). By contrast, in the Essentialist paradigm, laws are descriptions of the causal powers of things, and thus necessary (2001b, p. 215). Since the necessity of the laws derives from the essential natures of their relata $(2002$, p. 37), the truth-makers for the laws of nature are the fundamental dispositional properties $(2001 \mathrm{~b}$, p. 128$)$, and the identity of an object is tied to its behaviour in a way that is not contingent. Nonetheless, for Ellis, the manifestation of an object's power is linked, not only to the causal powers possessed by it, but also to structural properties and to relations between things. As Ellis notes, 'the laws of action of any causal power will always, or nearly always, be descriptions of the changes that would occur...in the relations between things, or in the structures of things, in response to various possible stimuli (which themselves will normally be just other possible changes in the relations between, or structures of, things)' (2001b, p. 137). 


\subsection{Causal Processes}

Ellis defines a causal power as a 'property that an object may have in virtue of a relationship between that object and a natural kind of process' (2001b, p. 206). This 'natural kind of process' is a causal process, involved in the display of causal powers (2001b, p. 206; 2002, p. 48). There are two different types of causal processes that Ellis sets out: causal interactions and energy transfer processes.

Causal interactions occur between and within (complex) particles, where the changes are discontinuous and instantaneous $(2002$, p. 83 ), as in atomic decay or spectral emission. Suppose that electron $\mathrm{X}$ of atom 1 drops from energy level A to lower energy level B. Also suppose that electron $\mathrm{Y}$ of atom 2 jumps from energy level $\mathrm{D}$ to a higher energy level $\mathrm{C}$. These changes of electron states indicate a causal interaction, but nothing can be said to have 'directly caused' either change, nor was there a continuous link between either A to B or D to C such that the change could be traced. (I parenthesise the topic of causation and systems as a whole with respect to these changes of state, since their discussion is not within the scope of this Thesis.)

A second type of process described by Ellis is that of energy transfer (2002, pp. 83-84), which may be initiated and terminated by the sort of causal interaction described above. For example, atom 1 undergoes the described change concerning electron X (from State A to State B), and this (somehow) gives rise to or initiates an energy transfer process, e.g. a photon being 'emitted'. Once initiated, no external force is necessary for that process to continue-it is inertial. Energy transfer processes conserve mass-energy, charge, spin, momentum and other universally conserved quantities (Ellis, 2002, p. 83). The energy transfer process terminates in absorption, let us say, at atom 2 where electron Y undergoes its change of state from D to C, and the photon is 'absorbed'. For Ellis, energy transfer is not a causal process in and of itself; rather, it is indeterminate in accord with Quantum Mechanics (2002, p. 84). So it is not that the energy transfer somehow 'caused' the change of state within atom 2 . Rather, within a closed system, causal interactions are followed by other causal interactions on a statistically significant basis, and energy transfer processes begin and end with such causal interactions. Altogether, a causal process is constituted by causal interactions plus energy transfer processes. 


\subsection{Summary and Conclusion}

This Chapter outlines the hierarchy of natural kinds that underpins New Essentialist claims for the existence of both categorical properties and dispositional properties, and sets the groundwork for the discussion to follow in Chapter 7. The categorical dimensions provide the structural circumstances that feature as factors in the operation of the causal powers. They direct and constrain what the causal powers can do by virtue of their quiddity, imposing effects by defining the ways in which their bearers can change in response to causal powers, whose range of effects is described by the laws of action and reaction. Ellis postulates a two-step description of causal processes, in terms of causal interactions and energy transmission processes. 


\section{CHAPTER 7}

\section{BRIAN ElLis:}

\section{NEW ESSENTIALISM - DISCUSSION}

In this Chapter, I examine the New Essentialist claim that fundamental categorical dimensions account for the structure that appears built-in to the universe. I argue that such dimensions, as formulated in the New Essentialist Theory, cannot sustain their identity in terms of quiddity. Moreover, their supposed mode of operation in directing and constraining causal powers renders their quiddity inseparable from their causal role. If quiddity is to be understood in terms of what there is to a property over and above its causal role, then it is not clear how attributing quiddity to these categorical dimensions can be justified, since it appears that what they are cannot be teased out from what they $d o$. Their identity, then, must be given at least partially by their causal role, weakening the claim that structure may be characterised purely categorically. The Chapter puts forward a thematic assertion that structure should be considered powerful rather than categorical.

\subsection{Quiddity}

The role of dimensions is to provide the circumstances in which the laws of action occur. How they do this is crucial to what the dimensions are. Ellis's categorical dimensions determine how the effects of causal power are distributed (Ellis, 2001a, 2008a). The effect of a causal process is to change the values of certain dimensions, described by Ellis as 'respects in which things may be the same or different' (2008a). These dimensions include quantities, shapes, duration, direction, spatiotemporal separation, position and time (Ellis, 2001b, pp. 136-138; 2008a). The constraint imposed by the dimensions - the fact that they are respects in which things can or cannot change - presumably affords their character as structural properties, and thus categorical. But this is compromised by the fact that Ellis also considers causal powers and capacities to be dimensions. His reasoning is that, like categorical dimensions, causal powers and capacities also represent 'respects in which things can be the same or different' (Ellis, 2008a). 
Given that both dispositional and categorical properties may represent dimensions, being a dimension per se does not render a property categorical; being structural or quiddistic does. This raises the question of how a categorical dimension differs from a dispositional or powerful one with respect to what role dimensions play in general. For example, if the role of dimensions is to fix the circumstances for the action of laws by virtue of being 'passively' structural or quiddistic, then an 'active' dispositional property, such as mass, cannot be regarded as a dimension in these terms. And conversely, if dispositional properties are counted as dimensions, as Ellis suggests, then fixing the circumstances for the action of causal power is not the only role that dimensions play. In this case, properties that provide the structural circumstances cannot be deemed categorical merely on the basis that they are dimensional; and it is clearly not the fact of being dimensional that is crucial to Ellis's argument for the existence of categorical properties.

Ellis claims that categorical properties are quiddistic by virtue of being structural; such that they contribute to the circumstances for the operation of laws of action according to what they are rather than what they do (Ellis, 2002, p. 69; 2008a). On these grounds, however, being a dimension does not justify counting structure as categorical rather than powerful. Instead, it is structure being quiddistic that purportedly renders it categorical. A difficulty-captured in the argument from quiddity - is encountered if the identity of a structural property is determined by means other than its causal role, since it would seem that, apart from some ability to engage in a causal process leading to our perception of such a property, we could not know anything about it. This problem appears to have been avoided by Ellis because his categorical dimensions do play a causal role (2005b, p. 470), albeit a passive one. They are 'factors' in the causal process and, as circumstances for the operation of causal powers, feature in the laws of action that describe these powers.

This causal role, while allowing room for structural properties to be recognised by virtue of their relationship with causal powers, problematises the claim for categorical dimensions being purely quiddistic. Given that categorical properties require a causal role in order to be recognised, it would appear that we cannot identify fundamental categorical properties in terms of quiddity alone. Ellis explicitly recognises this when he writes: 
It may be true that an atomic or a molecular structure of a given kind exists if and only if there is some atom or molecule that is disposed to behave in a certain way in appropriately specified conditions. But this is not what makes it an atom or molecule of this kind. Its essence is structural, not dispositional. It is, of course, only from the behaviour of an atom or molecule that we can infer its structure. But the structure exists independently of its disposition to behave in this way (2002, p. 69).

Here, what is postulated is the awkward notion that while the identity of categorical properties is supplied in terms of quiddity, we can only identify them in terms of their effects. As 'pure forms of physical structure', they have been described as restricting, constraining and informing the kinds of effects that causal powers can wield (Ellis, 2002, p. 174). Yet Ellis denies that they produce any effect that can be attributed to their own action. They do not 'resist, deflect or otherwise interfere with the actions of any known causal powers' (forthcoming). While determining where causal powers may exist and how they are distributed, they do so not as causal powers or, if my earlier argument holds, even by being dimensions per se; but by sheer dint of existing 'structurally'.

They purportedly fulfil their causal roles without acting, but this raises the question of how we might know about properties that are deemed to do nothing. How might their effect-contribution, including their ability to affect our perception of them, be achieved? Ellis explains that we can know about these entities, not by virtue of their own abilities, but because of the abilities and actions of the relevant causal powers. He claims that 'the physical causal powers always act to change the values of the dimensions of the things on which they act' (2008a), suggesting that the fundamental categorical properties might be discerned because they are respects in which things can change, and that this discernment is achieved by virtue of the causal powers of the objects that possess these categorical dimensions. Ellis notes:

Spatial properties, such as shape and size, are known to us because things of different shape or size affect us differentially. They produce in us different 
patterns of sensory stimulation, so that things of different shape and size look or feel different... But if spatial, temporal, and other primary properties and relationships are not causal powers, the question arises as to how we can know about them. We can know about them, we say, because of the dependence of the quantitative laws of action of the causal powers on these relationships. If the laws of action of the causal powers were independent of such factors as size, shape, direction, duration, spatio-temporal separation, and the like, then we could never know about them (2001b, pp. 136, 138).

In a recent communication, Ellis more explicitly describes and reaffirms how the categorical dimensions are discerned:

The categorical dimensions of things are made manifest to us, not directly by their own powers, (for they have none), nor by our own innate capacity of perception (for nothing can perceive a quiddity directly), but by the distributed causal power of the things that possess them, and our innate capacity to learn from experience about the shape of this distribution (2008a).

This explanation requires a relation between the categorical dimensions and the causal powers of their bearers. In short, things possess categorical dimensions that change in response to the action of causal powers, and these changes are perceived and interpreted by us, allowing us to infer the presence of the categorical dimensions. This seems to be what Ellis means when he writes, 'For the causal powers that stimulate our senses presumably all have constant laws of action that enable us ultimately to construct accurate neural maps of the locations of their sources, and hence of many of the categorical structures [sic] things that lie within range of our senses' (2008a). The upshot is that, in virtue of the causal role they play as they engage - via laws of action and reaction-with the causal powers, categorical dimensions escape the criticism that they are not discernible.

Earlier, I argued that being a dimension per se does not make a property categorical rather than dispositional. However, might being a dimension per se render a property powerful rather than categorical? The claim that quiddities can be known 
because of some causal role, even if passive, seems to raise the question of whether this role is fulfilled, not by virtue of the categorical dimensions being quiddistic, but because structure itself is powerful. The issue can be formulated in terms of teasing out what a property is from what a property does.

Let us suppose, as suggested by Ellis, that the role of categorical dimensions is to constrain and direct causal powers by limiting how they themselves can be changed. In this case, how changes can occur, and thus what the causal powers can do, seems 'built-in' to what the dimensions are. In this sense, the categorical dimensions are structural, yet play a causal, albeit passive, role. The problem is that the identity of these categorical properties is now determined not completely by what they are, but also by what they do. Ellis suggests that we recognise at least certain categorical properties through common patterns of spatiotemporal relations (forthcoming), where these patterns are recognisable, not because of the categorical properties of the bearers, but because of essential dispositional properties that these bearers also possess. Thanks to these latter properties enacting patterns of behaviour upon the former, we discern the existence of categorical properties. However, I maintain that the categorical dimensions have some effect if the patterns arise by virtue of their evidently interactive presence; and this appears to render them powerful in some way.

A further doubt about the purported quiddity of categorical dimensions is raised by considering what we really mean when we talk about the units of dimensions; say, mass or distance. ${ }^{21}$ Certain quantities in fundamental physics can be reduced to dimensionless numbers, dispensing with units altogether. Choice of units such as the second or the metre is often a matter of convenience and to a large extent reflects accepted conventions of physical theory. For example, as James Hartle notes, the second is defined as 'the time required for exactly 9,192,631,770 cycles in the transition between the two lowest energy states of a Cesium atom'. Employing the observation that the speed of light is the same in all inertial frames of reference, the metre is then defined as $1 / 299,792,458$ of a second (Hartle, 2003, p. 541). We have separate units for mass, length and time because our prior physical theories used independent standards for these quantities. The metre, for example, was defined by

\footnotetext{
${ }^{21}$ I wish to thank Brian Ellis for this suggestion.
} 
the distance between two marks on a particular bar, and the second was defined as a certain fraction of the mean solar day. Developments in physical theories, however, have come to show the interdependence of dimensions as measurement conventions change to reflect updated information. $\mathrm{E}=\mathrm{mc}^{2}$ is a case in point. As absolute quantities, both the speed of light and Planck's constant are frequently assigned the value of unity. The 'kilogram' has been traditionally defined as 'the mass of the block of metal kept in the Bureau International des Poids et Mesures, in Sèvres' (outside Paris). Today, as Hartle notes, the kilogram can be defined in terms of distance: 'with confidence in the equality of gravitational and inertial mass, general relativity, and access to precise enough measurements, the kilogram could be defined as the mass of a sphere such that a test mass completes a circular orbit of radius $1 \mathrm{~m}$ in some defined number of days' (Hartle, 2003, p. 542). As Ellis notes in personal communication (Dec 2009), if it is in principle possible to measure distance, say, in terms of kilograms, then it might be also possible for all quantities to be measured in terms of dispositional ones. If so, then all quantities could be seen as derived dispositionally. This principle can be taken further to suppose that it might be equally convenient (or inconvenient) to measure all quantities categorically, depending on instrumental purposes. This situation raises the question of whether the categorical-dispositional divide is best viewed as a supervenient, higher-order distinction which, albeit intuitively appealing and instrumentally useful, embodies no ontologically-robust division of reality. (This issue is taken up again in Chapter 14 of this Thesis.)

It is arguable that if 'quiddity' means what a property is over and above its causal role, then no such pure form of quiddity exists. However, rather than assuming the relevant property to be therefore dispositional, an alternative-and perhaps better-solution might be to recognise such properties as 'powerful', a topic also taken up again in Chapter 14. In brief, unlike the 'power-qualities' put forward by Charlie Martin and John Heil in their Identity Theory of Properties (Heil, 2003a; Martin, 1996b, 1997), I suggest that power is aligned with neither categoricity nor dispositionality. Properties that are powerful might be best described as those that both are, and yet $d o$, merely by virtue of being. Although powerful, these may also be described as structural. Such properties are not categorical because they are not purely quiddistic, but I resist viewing them as dispositional because they are ontologically- 
robust, and hence always manifesting simply by virtue of existing. Molnar proposes that one might allow such properties 'full ontological status on par with all of the paradigms of respectable existences' (Molnar, 2003, p. 141). This requires powers to be more than mere possibilia, since it involves actuality in the sense of ontologicalrobustness.

One important reason Ellis provides for rejecting the consideration of structure as powerful rather than categorical is couched in the form of a neo-Swinburne regress argument. The Swinburne regress objection is that a purely dispositional world ultimately lacks the resources to allow for the detection of properties and their effects. As noted earlier, Richard Swinburne argues that a regress occurs for such worlds because powers are only recognised by their effects, but that these recognised in terms of the properties they involve. Since properties are themselves nothing but powers, then effects must be recognised by effects which must be ultimately recognised by effects, and so on; but at no stage do the required properties appear. Swinburne suggests that this regress can only be broken if there is something more to properties than powers (1980a, p. 317). The idea of structure being categorical is thus driven by calls for the effects of causal processes to be directly observable at some point, for which categorical properties are purported to be necessary; supposedly affording direct perception of effects. Ellis claims that, at some point in the causal chain, changes must occur in 'directly observable dimensions of things' (2008a). He further notes that, although causal powers also give us direct knowledge of the world, quiddities 'are among the most direct objects of knowledge that we have of the world' (forthcoming). However, I suggest that almost the opposite seems to happen if, as noted earlier, these are observed only indirectly via patterns of distributed causal powers.

I have argued that we are: i) not able to tease out what a property is from what it does; ii) on the understanding that certain absolute physical quantities permit reinterpretations of measurement, not able to clearly differentiate between categorical and dispositional quantities; and iii) not able to directly detect quiddities, which leaves the Swinburne regress unresolved. 


\subsection{Causal Laws and Location}

Do the laws offer some way to tease apart quiddity from the causal role of the categorical dimensions? As noted in Chapter 6, Ellis's causal laws are metaphysically necessary descriptions (Ellis, 2001b, p. 116; 2002, pp. 88, 100-101) of the essential natures of the kinds of things in the world (Ellis, 2002, pp. 59, 88, 101). These laws of action and reaction specify the categorical dimensions (Ellis, 2005b, p. 470), describing the effects of change as understood in terms of the modification of dimensional values (2001b, p. 206; 2008a).

However, relying on these relations - the laws of action and reaction-to explain why the dimensions change as they do does not rescue the dimensions from being essentially powerful. In $7.1 \mathrm{I}$ have argued that having some causal role excludes a strictly quiddistic essential nature that is inert in terms of causal agency. There is no account of how a causally inert property may undertake a causal role. This highlights the case that what the dimensions are cannot be altogether teased out from what they do. It can be further argued that the constraining and patterning of the causal powers cannot be relegated to the laws of nature, since these laws are merely descriptions of the workings of the essential natures of their relata. Were the laws contingent, representing something over and above the natures of their relata, it could be argued that they would somehow carry the weight of the causal role. But the laws are necessary, precisely because they are nothing more than descriptions of the essential nature of their relata, which denies their utility in separating out what the dimensions are from what the dimensions do.

Armstrong raises this issue, pertaining to necessary versus contingent laws, through an example concerning spatiotemporal distance. He argues that if, via their causal role categorical properties are included in the specification of the action of causal powers, provided by the laws, then these laws must contingently exist rather than necessarily exist. Since Ellis's categorical dimensions are very similar to the Sproperties utilised in George Molnar's discussion of Shoemaker's Causal Theory of Properties (Molnar, 2003, pp. 160-164) (presented in Chapter 5 of this Thesis), the example that Armstrong employs is derived from Molnar's argument: 
Let us consider two particulars, each having some mass, which attract each other according to some formula, say the Newtonian inverse square law. The masses we think of as powers, but the distances are non-powers, given the theory we are examining. But the forces generated between the two particulars vary inversely with the square of the distance. Have not the two particulars got to 'know', as it were, at what distance they are from each other if they are to exert the right amount of force on each other? Struggling with this difficulty, Molnar speaks of the mass-properties that are powers being 'sensitive' to the distance. But to be sensitive to something is to be able to pick up signals from it. Sensitivity is dispositional/causal in its essence. For the particular case this means that Molnar is conceding that distance actually has some sort of causal efficacy. So for him causal efficacy is not confined to powers. And that causal efficacy will presumably be contingent, not necessary. (And if it is necessary, it cannot be a transparent necessity.) So for Molnar and Ellis it seems that there will be an element of contingency in the Newtonian gravitational law (2004b, p. 140).

The causal role played by distance implicates it in the generation of effects or manifestation of causal power. Since these structural properties (and relations) are said to be categorical, the contribution that they make to such effects cannot be necessary; rather, it must be contingent. Hence, the effects of causal power cannot be necessary; rather, they must be contingent, in which case the laws that describe the causal process cannot be necessary, as Ellis claims; rather, they must also be contingent. If Ellis wants to claim a causal role for categorical properties, then his laws must be contingent, given that the properties feature as the relata of the laws. Necessary laws require that all causal contributions are necessary, and this rules out categorical properties. Armstrong writes:

You will see that I am putting a destructive dilemma to Ellis. Either every factor involved in a causal action is a power, and then the Swinburne objection kicks in; or else there are non-powers involved. If non-powers are involved, 
then their causal contribution is contingent. And then the effects will not be necessary (2001, p. 170).

Ellis's response is to deny that there are only two possibilities - that structural properties contribute to the effects necessarily, or they contribute contingently. $\mathrm{He}$ puts forward a third possibility; that something like distance does not directly contribute to the effect. Rather, distance is one of the dimensional quantities, itself affected by the action of causal power, and in turn affecting causal power. But causal power, not distance, is the driver of the effect. To explain: Let D (the spatiotemporal relation of distance) be the structural circumstance in question; A be some object; and $\mathrm{C}$ be a causal influence. Ellis writes:

This ingenious argument rests on the assumption that the laws of action of the causal powers are not functions of the circumstances in which they act. If the effect on some $\mathrm{A}$ is a function of its distance $\mathrm{D}$ from a causal influence $\mathrm{C}$, then Armstrong thinks that there are only two possibilities: Either $\mathrm{D}$ is a contributing cause to the overall effect, which makes its contribution by necessity, or it is not. If it is, then I am caught up in Swinburne's regress. If it is not, then the distance must make its contribution contingently. However, there is another possibility. One can simply deny that D is a contributing cause. In my view, D is a dimension of the causal set-up. It is one of the categorical properties that both affects and, in this case, is also affected by, the causal power (2002, p. 172).

As Armstrong sees it, relations between entities are either necessary, in the case of causal powers being specified by the laws; or contingent, if the dimensions are included in the focus of specification. However, Armstrong sees an incompatibility between laws as specifying both causal powers and contingent circumstances and those laws also being necessary: 'It looks as though these structural properties must have some "causal role", he writes, "And will they not have that role contingently only? Not being powers, they do not necessitate any particular causal role' (Armstrong, 2001; 2002, p. 170). 
In addressing Armstrong's criticism, Ellis argues that, although the objects involved in the causal laws operate within contingent circumstances, they all have causal powers and dispositional properties as part of their essential natures. The necessity of the laws derives from these 'essential natures of the things on which they are said to operate' (Ellis, 2002, p. 37). He has further argued that the passive causal role attributed to categorical dimensions as 'factors' in causal processes can be differentiated from the active causal role of causal powers (forthcoming), and this he uses this differentiation to claim for necessary laws rather than contingent ones. Hence the argument appears to hinge on the active versus passive distinction. I argue, however, that the intrinsic-extrinsic differentiation is the important and relevant driver in this dispute. Defending my claim involves two related observations: First, spatiotemporal properties, such as distance, feature in laws of action more generally, whereas causal powers are more specific. Second, for ontologies that posit distinctness of objects, spatiotemporal properties or relations, such as location or distance, are extrinsic with respect to the objects bearing the relevant causal powers.

Ellis (forthcoming) provides the example of a weight suspended above ground level with the causal power to compress, stretch or pull things by way of potential energy. How the power manifests depends on the relevant circumstances, e.g. where the weight is in relation to other things, how it is fixed in position, and so on. The law of action concerns the effect of the weight in terms of the strength of the causal power as a function of the dimensions and initial circumstances. Ellis notes that all such laws of action are quantitative, depending on the magnitude and location of relevant powers; and that all involve one or more categorical properties, these categorical properties comprising the dimensions or circumstances in which the powers operate. The spatiotemporal property of location (assuming it can be considered a property) is paradigmatically categorical for Ellis (forthcoming), and importantly, all causal powers are located. This is one reason why the laws of action describing causal processes all include categorical dimensions.

The term 'location' is encompassed by the more general idea of spatiotemporal relations. In keeping with Armstrong's example, distance describes the location of one object with respect to another in terms of spatial separation. Location can also relate an object and a spacetime point within a frame of reference; even an 
absolute frame of reference, such as in the example cited by Ellis whereby a location, if emptied of all causal powers, would still be a location. Ellis notes that all instances of causal powers have specific locations, which are had contingently. He supplies the example of specific instances of gravitational mass (forthcoming). Objects are located here or there, but might have been located otherwise, or subsequently change their location through time. Thus the property of gravitational mass, although essential and intrinsic to those objects possessing it, is borne by objects that are nonetheless located contingently with respect to some spacetime frame of reference. By contrast, instances of location are said by Ellis to be necessarily where they are.

Location might be conferred with meaning in terms of the relation between an object and an absolute spacetime through which the metric for a fixed 'background' structure is presupposed (Kribs \& Markopoulou, 2005, p. 4). Alternatively, from a relationalist perspective, location has meaning only with reference to physical entities. On one hand, if location is derived via reference to a fixed background, then as Alexander Bird (2005a; 2007, pp. 161-168) and Stephen Mumford suggest (2004, p. 188), this may constitute merely a choice of theoretical perspective. According to General Relativity, spacetime is not absolute, while various theoretical models in physics and in metaphysics treat spacetime as emergent (Bilson-Thompson et al., 2009; Bilson-Thompson et al., 2007; Harré, 1970; Harré \& Madden, 1975; Smolin, 1997, 2000, 2006). On the other hand, if location is derived only with respect to the contents of the universe, then in keeping with the contingency of objects' locations, instances of location in general should also be deemed contingent. Moreover, even if it were hypothetically possible to remove all causal powers from a certain spacetime region, as Ellis suggests, the location itself would nonetheless be derived or 'framed' in relation to neighbouring objects.

Ellis attempts to base the categoricity of location on a contrast between the necessity of location instances and the contingency of causal power instances. The above argument amounts to proposing that this contrast only holds for theories that adopt a fixed spacetime background. I also earlier commented on why an active versus passive distinction between causal powers and categorical dimensions is unpersuasive. However, a distinction based upon whether a property is intrinsic or extrinsic to an object, whose causal powers feature in some law of action, is an option 
for those ontologies that posit distinct objects. Taking distance, for example, Ellis argues that its being a factor in the outcome of a causal process-e.g. 'living a long way from Sydney prevents one from walking there'- does not itself constitute a causal power. We may think of causal powers in terms of dispositional properties, the possession of which bestows upon their bearers the ability to act in certain ways depending on the essential nature of these properties. Then distance does not bestow any particular ability (or in this case disability) upon the walker. Given that it features in many other causal laws, it is not an essential property or causal power intrinsic to the walker. Rather, since distance is extrinsic to the walker, it is a non-essential, categorical property, only contingently related to the walker. Nonetheless, it features in the law in question, playing a role in conjunction with other more specific powers that are intrinsic to the walker, such as endurance, muscular power and cardiovascular condition. Similarly, in the example of the weight mentioned earlier; its location with respect to other objects contributes to the circumstances within which its causal powers operate, and which contribute to the laws of action of those powers. However, because its location is given with respect to other objects that are external to the weight itself, this cannot be an essential property of the weight, and hence cannot be a causal power of the weight. It must, instead, be a contingently existing, categorical factor that features in the laws of action specifying the effect of the weight.

Ellis defines a causal power as follows: 'Any quantitative property $\mathrm{P}$ that disposes its bearer $\mathrm{S}$ in certain circumstances $\mathrm{C}_{0}$ to participate in a physical causal process, which has the effect $\mathrm{E}-\mathrm{E}_{0}$ in the circumstances $\mathrm{C}_{0}$, where $\mathrm{E}$ is the actual outcome and $\mathrm{E}_{0}$ is what the outcome would have been if $\mathrm{P}$ had not been operating' (forthcoming). A physical causal process is defined by Ellis to be an energy transfer from the state of one physical system to another, so as to bring about a physical change in the system that would not have occurred in the absence of that physical causal process (forthcoming). This description outlines two criteria for being a causal power: It must dispose its bearers to be involved in causal processes that i) involve transfer of energy; and ii) would thereby make a difference in outcome so long as the circumstances remain constant. (A causal process builds-in the idea that energy transfer occurs between states of different systems, although I see this as including energy transfer between different states of sub-systems of complex systems.) 
Applying this description of causal power to the Sydney example: Living a long way from Sydney disposes me, in certain circumstances, to not walk to Sydney, which alters the outcome of whether or not I go there. No transfer of energy takes place, either actually or counterfactually, and so 'living a long way from Sydney', i.e. distance from Sydney, is not a causal power. But this example is complicated by being phrased negatively. Here is a parallel, but positive example: Living close to a dairy disposes me to buy milk there rather than go without milk. Here, energy is transferred in the process of walking to the dairy and bringing milk home. A physical change occurs, fulfilling one requirement for my location to be a causal power. The second requirement is that the presence or absence of closeness makes a difference to the outcome. Were I living further from the dairy, I would have no milk. So this criterion for distance being a causal power is also met.

According to the definition of a causal power, therefore, it is not clear from this example alone why distance should be treated as a factor or circumstance in my having milk rather than as a causal power. The reason could be that choosing to buy milk is typically seen as a contingent matter, even with the relevant circumstances in place. The supposed contingency is tied to the scenario whereby myself, my home, the dairy and the milk are all counted as distinct objects rather than constituting a single system. My buying milk is accordingly considered contingent by the Humean Principle of Independence, which disallows necessary relations between distinct objects (see, Armstrong, 2000, p. 8). Causal powers, however, do not represent contingency in this way. The power to crush an object under a weight, for example, would operate necessarily, providing that the circumstances specified in the laws of action of that power were in place. (I am putting aside probabilistic causal powers or propensities for the purpose of this example.) Thus, the claim that distance is merely a factor rather than a causal power depends on whether the separation between objects is viewed as extrinsic and contingent or as intrinsic and essential.

How might we observe distance as intrinsic rather than extrinsic? Suppose Mars and Venus were 50 million miles apart. The two masses could be counted as causal powers with specific locations (possibly identifiable with particular singularities in the gravitational field). But if the situation is viewed from a fieldtheoretical perspective, taking into account the entire region's contours of 
gravitational potential, then all the field contours throughout the 50 million-mile region are directly involved in how the whole set of field contours will behave- and this is the only relevant behavior that will take place. Given any contingent variation in the local field contours, such as constituted by the presence of Earth, for instance, the field contours of the entire region would then behave differently. In fact, given the field topology at any time, and its subsequent behaviour, we could retro-determine the distance between Mars and Venus. (The topology and behaviour of the topology need not explicitly incorporate distance). This perspective implies that the relevant causal power (in this case gravitational power) is not really at any particularised locationbut exists everywhere throughout the region of interest. At any time, any difference in the field contours of the whole region would cause different behaviour, and since distance is an intrinsic aspect of the field contours, it thus corresponds to causal power. Clearly, the distance could be retro-determined in various ways - in terms of other causal powers and laws - which might be taken by some theorists ${ }^{22}$ to suggest its ontic independence. For example, the distance between any two charges could be retro-determined from the topology of the electromagnetic field at a given time, along with that field's subsequent behaviour. (Naturally, at this scale, the scenario is extraordinarily complicated by the phenomena of quantisation.) Both gravitational and electromagnetic scenarios will determine the same distance, but this simply indicates an intrinsically deep connection between the scenarios. It highlights that the singularities of gravitational fields could simultaneously be the singularities of electromagnetic fields. (Of course, it is commonly, if optimistically, anticipated that a unification of all the fundamental forces will be discovered.)

Perhaps being an extrinsic, contingent relation between distinct objects or states, versus being an intrinsic, essential property, amounts to the difference between what is deemed a categorical dimension and what constitutes a causal power. If so, then the difference is theory-bound rather than ontologically-robust. Say, for example, my home, the dairy and the land stretching between them are viewed as part of the same, very complex system rather than as distinct objects. In this case, just as in the Mars-Venus case, distance (as 'size') is an intrinsic property. Likewise, the weight, the wire and the object situated for compression may all be considered a single system

\footnotetext{
${ }^{22}$ This was a point made to me by Brian Ellis in personal communication.
} 
to which the distance between the weight and object is intrinsic and essential. The argument between Ellis and Armstrong introduced and discussed earlier in this Section, concerning necessary versus contingent laws, thus hinges on the status of circumstances as extrinsic or intrinsic to the system described by the laws in question. The key point of difference between Armstrong and Ellis is subtle. Armstrong's contention involves the assumption that the laws described by science are derived via observations, that are made among diverse circumstances in all of which the respective causal powers operate. The specification of laws acknowledges the contingency of circumstances from which they are derived. Therefore, in describing systems, laws are based on circumstances that are intrinsic to the nature of those systems. By this reasoning, since the nature of any system is at least partially contingent, the relevant laws must also be contingent.

Ellis's approach, to the contrary, assumes the circumstances to be extrinsic to the objects whose causal powers are described by the laws. These laws, which he describes as necessary, can be best characterised, I suggest, as 'unsaturated' of circumstance. One way to do this would be to separate out and exclude the plethora of possible circumstances in which a causal power might operate. In practice, from the myriad observations of a certain causal power operating in various circumstances, we extract a common thread that depicts the essential operation of the causal power in question. Formulated by this means, such 'laws' would be ideal ones, specifying the essential nature of causal powers, and could in principle be considered necessary. However, applying the term 'law', as above - to describe the essential nature of a causal power - risks confusing it with the term 'law' as a description of the operation of causal powers in specific circumstances. I suggest that such unsaturated lawsEllis's necessary laws describing the essential nature of objects in terms of their causal powers - conform better to what Rom Harré calls 'generative mechanisms' (Harré, 1970; Harré \& Madden, 1975), a topic covered in more detail in Chapter 12 of this Thesis. This distinction might permit Ellis to more readily differentiate ideal descriptions of the essential operation of causal powers, aligned with necessary laws, from those descriptions that specify the operation of objects in specific circumstances, described by contingent laws. This might provide a resolution to the impasse between 
Armstrong and Ellis on the nature of laws; while allowing contingent circumstances to be represented as functors in the everyday actions described by science.

This section began by asking if laws provide some way to tease apart quiddity from the causal role of the categorical dimensions. On one hand, I propose that the unsaturated necessary laws described above-ideal specifications of the causal powers-have nothing to say about the circumstances, so cannot explain how the categorical dimensions, as pure quiddities, are recognised. On the other hand, if distinguishing unsaturated laws from laws that include specification of circumstances is rejected in favour of claiming that all laws must specify circumstances, then Armstrong seems justified in approaching such laws as based on underlying contingency. In this case, the causal role of the circumstances is recognised, although the laws must be admitted as contingent. If the causal role is recognised as part of the specification of the laws, this supports my earlier conclusion that what the categorical dimensions are cannot be teased out from what they do, and thus cannot be purely quiddistic.

The upshot is that purely quiddistic properties are unrecognisable and therefore surplus to a theory. Properties that play a causal role- even a passive oneeither comprise part of the specification of the laws or they do not. If they do, then the laws themselves must be contingent, as per the argument above. But if the laws are given as unsaturated descriptions of the causal powers, then while being properly considered necessary, they have nothing to say about the causal role of the categorical dimensions or how the purported quiddities are recognised.

A solution might be for Ellis to adopt two types of laws into his system: those that he considers contingent, and which describe the essential natures of powers as they feature in their operation in the physical world; and those that are necessary, which are ideal descriptions of the essential natures of objects, and which merely encounter and incorporate contingency upon being instantiated. An alternative solution would be to abandon categorical dimensions in favour of powerful ones. However, such a view culminates in a Foundation-Monism, the stance that I defend in more detail in Section 4 of this Thesis. 


\subsection{Summary and Conclusion}

The New Essentialism advocated by Brian Ellis proposes the ontologically-robust existence of both dispositional and categorical properties. The two are combined such that fundamental categorical properties - termed 'categorical dimensions' or 'fundamental structural properties'-fulfil a passive causal role in constraining the effects of active causal powers. I have argued that this distinction is unpersuasive, since although a passive role allows categorical dimensions to be recognised, it compromises the claim for their characterisation as purely quiddistic. Hence, we are not able to properly tease out what a property is from what it does. This observation is reinforced by the fact that absolute physical quantities permit re-interpretations of measurement that remove a clear differentiation between categorical and dispositional quantities. Moreover, Ellis's assertion that structure is categorical is partly driven by the Swinburne regress argument, which calls for the effects of causal processes to be directly observable at some point; and which claims that categorical properties are required for this to occur. However, as Ellis notes, categorical dimensions can only be observed in virtue of perceiving a pattern of distributed causal powers. This implies that categorical properties must be either perceived indirectly or inferred from the way things behave. Thus, arguments for fundamental structure given in terms of categorical properties fail to discharge the Swinburne regress. On the back of my argument that the causal role of categorical dimensions-what they $d o$-is inseparable from what they are, I have suggested that the dimensions should be considered powerful rather than purely quiddistic, although a detailed characterisation of powerful structure is postponed until Section 4 of this Thesis.

The second topic raised in this chapter concerns the laws of action and reaction and claims for their being necessary rather than contingent. Ellis claims them to be necessary, since they describe the essential natures of the causal powers relevant to their specification. I have argued that the intrinsic-extrinsic division is a primary determinant of whether spatiotemporal properties and relations, such as location and distance, should be incorporated within the specification of the laws. Ellis has argued that spatiotemporal properties and relations are categorical. In such light, if they are counted as intrinsic to the system whose causal powers are described by the relevant laws, the laws would appear to be contingent. I have suggested one way around this 
difficulty by recognising two different types of law: Ideal laws that are unsaturated, derived by extraction from the circumstances and thus describing causal powers alone. These laws may be considered to be necessary, since nothing categorical is involved in their specification. The second type of law corresponds to instances of ideal laws that specify not only the essential natures of the causal powers, but also the action of the causal powers in particular circumstances. Where these circumstances are deemed categorical, then in keeping with Armstrong's perspective, I have suggested that these such laws should be deemed contingent. As I see it, purporting these two types of laws does not contradict the central claims of New Essentialism, that the causal powers are essential to the objects that possess them, although it does provide for an answer to the dilemma that Armstrong raises concerning categorical properties entailing contingent laws. 



\section{CHAPTER 8}

\section{Ullin Place:}

\section{CONCEPTUALISM - OUTLINE}

In Dispositions: A Debate (Crane et al., 1996), Ullin Place engages with David Armstrong and Charlie Martin concerning dispositions. ${ }^{23}$ The Conceptualist view expressed by Place is outlined in this Chapter. An inventory of the contents of the universe according to Place comprises four categories: concrete particulars (i.e. categorical objects, entities or substances); dispositional properties of, and categorical relations between, concrete particulars; situations (events or state of affairs); and dispositional properties of, and categorical relations between, situations. Place shares Armstrong's view that the world is composed of concrete particulars that are bearers of properties; and that there are no such entities as 'bare' concrete particulars, or bare properties and relations (1996b, p. 53).

Place's Conceptualism posits the existence of purely dispositional properties, which he describes as consisting of simply the property-bearer having a 'projection' or 'orientation' towards possible future possible or past counterfactual events:

[D]ispositional properties are 'pure' in the sense that they do not consist in anything over and above a projection or orientation (there's no avoiding metaphors here) of the properties bearer towards what would happen, if in the future certain conditions were to be fulfilled. They are not pure in the sense of H.H. Price's supposition that there are dispositional properties which have no 'categorical basis' whatsoever. All such properties, according to Place, have a basis in the structure, either macro or micro, of the property bearer. It is just that, on this view, the dispositional property and its structural basis are two distinct and causally related things, not one and the same thing (Place, 1996e, p. 119).

\footnotetext{
${ }^{23}$ The narrative style of Dispositions: A Debate has authors referring to themselves in third-person.
} 
These dispositional properties are caused to exist by the structures of the objects that possess them. In keeping with Hume's Principle of Independence (discussed in Chapters 2 and 3 of this Thesis), they are also distinct from their structural basis, and thus not reducible to their causal basis. The structures of objects include both dispositional and categorical components (Place, 1996e, p. 118), although no pure categorical properties are conjectured to exist (Place, 1996c, p. 27). The idea of 'categorical' can be contrasted with the notion of future possibility and with past counterfactual representations of the dispositional (1996b, pp. 60-61). Place's distinction between what it means to be categorical or dispositional carries a slightly different nuance to that of Armstrong, Shoemaker or Ellis. For Place, the term 'categorical' emphasises the actuality of an existent; thus the term 'categorical' can only be properly applied to an entity existing fully in the present moment, 'having no projection beyond the here and now' (1996e, p. 115). Properties are what exist through time; thus they denote the possibilities a thing had in the past and will have in the future. Since this fact about properties coincides with how Place defines dispositionality, it rules out the existence of purely categorical properties (1996c, p. 21).

Unlike Armstrong, whose truthmakers for dispositions reside in the categorical microstructure of property-bearing objects, Place conceives truthmakers for dispositional ascriptions to be nothing more than the possession of a dispositional property by an object (1996c, p. 20). When we ascribe brittleness to a tumbler, for example, the truthmaker for that statement is the possession by the tumbler of the dispositional property of 'brittleness'. However, because the nature of the dispositional is given, contra categoricity, in future possible and past counterfactual terms, the truthmakers for dispositional properties, themselves, are just counterfactual states of affairs. This view has similarities to the Phenomenalism concerning dispositions proposed by Gilbert Ryle (discussed in section 2.1 of this Thesis). Place's dispositional properties take their truthmakers to be predictive descriptions of behaviour:

On this Rylean view, it is a matter, not of anything that is happening or is the case in the here and now, but of what would happen or, in the counterfactual 
case, would have happened if certain conditions were to be or had been fulfilled. If that is correct, there is nothing more to the truthmaker of a causal counterfactual than what may quite properly be called a 'counterfactual state of affairs', a state of affairs whereby certain predictions and counterfactual retrodictions of which the counterfactual in question is one are true of the owner of a dispositional property (1996c, p. 21).

An important difference between Ryle and Place, however, is that Ryle's dispositions are nothing more than predictive descriptions of behaviour (1949, pp. 43, 123), while Place's dispositions derive some kind of ontological reality in virtue of being caused by a presently-existing structural basis. For Place, the possession of a disposition is a matter of being able to answer the question, "what would happen if this or that circumstance were the case?' and have the answer causally connected to the structural basis of the object in question.

Place's objects are combinations of categorical and dispositional components. Since his defining characteristic of the categorical is ontologically-robust existence in the present, whatever about the microstructure that fulfils this description constitutes the categorical component. This includes concrete particulars (e.g. property bearers), permitted the status of being purely categorical since they exist at each present moment. Possessing properties, however, occurs across a period of time, and this idea of past and future 'existence' of properties is captured in the notion of the dispositional (1996c, p. 22). Although Place does not allow purely categorical properties, since he does admit purely categorical relations, including spatiotemporal relations between the parts of a (complex) particular and between it and other objects in the world (1996c, pp. 22, 27; 1996e, p. 115). A rationale for Place's denying the existence of categorical properties while allowing categorical relations is in the context reduction; we can account for structural properties like shape and size (paradigmatic categorical properties) in terms of relations. Place writes that, 'the external shape and internal structure of a concrete particular, appear to reduce on analysis to spatial relations between the concrete particulars which make up the whole' (1996c, p. 27). I construe Place's relations to conform to his definition of categoricity by corresponding to processes that occur in each present moment, and not 
persisting through time in the same way that properties so persist. Altogether, the microstructure of objects can be characterised as a complex of (1996e, p. 116):

i) the "purely categorical existence of the parts of which the substance is composed';

ii) the purely categorical existence of spatial relationships between parts; and

iii) the existence of the dispositional properties of the parts that interact with one another and maintain the integrity of the whole.

Place views dispositional properties as essential to the existence of causal relations. He writes, 'Place not only allow[s] dispositional properties to play a basic role in causality, he insists that the existence of a causal relation, any causal relation, depends on the coincidence of two causal factors, one categorical/structural, the other dispositional/modal' (1996e, p. 115). Microstructural properties cause the existence of dispositional properties, which govern interactions of the bearer with other objects. A dispositional property is not - as Armstrong circa 1997 holds - to be contingently identified with the microstructure or the state of the microstructure. Rather, for Place, the microstructure causes the tumbler to possess the property of brittleness, and it is this property that causes it to shatter when struck under particular conditions. Thus the microstructure (as cause) stands in a causal relationship to the dispositions (as effect) (1996c, p. 20). Place subscribes to the 'Humean view' that causal relations hold between distinct existences, claiming the doctrine to be a crucial premise in his argument for the ontological independence of dispositional properties from their microstructural bases (1996a, p. 154; 1996c, p. 30). For Place, the causal relationship between the state of possessing the dispositional property and the state of the microstructure are two distinct states of affairs (1996c, p. 20). He provides three arguments for the distinction:

i) Dispositions are of a different category to the categorical and have different roles, different characterisations and different features and these differences are unbridgeable (1996b, p. 60).

ii) The microstructure concerns the internal space of an entity, whereas the dispositional is located outside (external) to an entity. 'Roughly speaking', writes Place, 'we can say that the microstructure of an entity is 
inside the entity, whereas the dispositional property, in so far as it is located anywhere, is outside the entity at its point of interaction with other things' (1996b, p. 61). Hence the two cannot be one and the same thing.

iii) Differences in causal role; microstructures stand as cause to dispositions, and following Hume, must therefore be distinct from them (1996b, p. 62).

In summary, dispositional properties are distinct from the structural basis that cause them. Truthmakers for dispositional ascriptions are given in possible future or past counterfactual terms. The structure underlying dispositional properties is comprised of both structural and dispositional components (Place, 1996c, p. 26). 


\section{CHAPTER 9 \\ UlLin Place: \\ CONCEPTUALISM - DisCUSSION}

This Chapter discusses the Conceptualism of Ullin Place, focusing on two main objections: First, the lack of an independent truthmaker for ascriptions of dispositions; and second, microstructures causing the dispositions of their bearers, while remaining distinct from these dispositions.

\subsection{Truthmakers for Dispositional Properties}

Tim Crane notes that, for Place, the dispositional is distinct from the categorical and that both are equally real (1996, p. 8). If dispositional properties are 'real' - as real as some believe categorical properties to be-then what are they? Although Place's dispositions are not given a purely phenomenological account in the same manner as Ryle's, Armstrong argues that their lack of an 'ontologically-robust' truthmaker amounts to such (1996a, pp. 15-16). The only truthmaker that Place can provide to support his dispositional properties is a counterfactual state of affairs or fact. The appearance of dispositions being ontologically-meaningful, is gained by conflating the linguistic counterfactual with its existence in some 'real' sense. However, the Truthmaker principle requires truth to depend not on some further statement, but ontological ground. A merely counterfactual state of affairs or fact to support the ascription of dispositional properties appears in the same form as that which it is intended to warrant. In this case, a counterfactual statement is supplied to warrant a statement about dispositional properties. For Armstrong, the two far too closely resemble each other to allow suitable truthmaking. He writes that, 'On this view, the counterfactual statement has a form that pictures rather directly the form of a certain portion of reality: the counterfactual state of affairs' (1996a, pp. 15-16).

Place agrees with Armstrong that should a counterfactual state of affairs be defined as a 'fictional event', then it would indeed be absurd to claim it as part of reality (1996c, p. 20). Place's reply is that his counterfactual state of affairs is not 
fictional. He notes that the counterfactual statement and the state of affairs that makes it true are two different things; the former is non-existent but the latter does exist:

But this is precisely the difference between a simple categorical statement of the cat is on the mat variety and the case of counterfactuals, subjunctive conditionals, law statements, etc., where what the statement depicts and the actually existing state of affairs which makes the sentence true are two different things; necessarily so, because in these cases the event or state of affairs depicted does not exist, has not existed and may never exist, whereas ex hypothesei the state of affairs which makes the counterfactual true most certainly does (1996c, p. 20).

Place further objects, claiming that Armstrong's criticism assumes that proper truthmakers need to be categorical or somehow ontologically-robust (1996c, p. 19). His defense relies on denying that 'pure' categorical properties exist. Since the microstructure of any property-bearer is comprised of categorical relations and categorical particulars bearing their dispositional properties, the microstructure is not itself purely categorical (1996c, p. 22). Hence, Place questions whether a microstructure can be properly categorical. Since Place denies pure categorical properties, Armstrong begs the question against him in requiring categorical truthmakers. Place further points out that Armstrong's microstructure, as truthmaker for dispositions, is no more ontologically robust.

I will leave aside Place's argument that Armstrong cannot supply a categorical truthmaker, since Place depends on his own formulation of microstructure to make this assertion. Since Armstrong's version of microstructure entails purely categorical properties and contingent laws, Place similarly begs the question against Armstrong. Place's further responses focus on the claim that non-categorical truthmakers are equally as viable as categorical truthmakers, since they are equally real. Consider, for example, an existent state of affairs such that if a certain tumbler were struck in the right circumstances it would shatter. Place's contention is that the reality of this state of affairs acts as truthmaker for the counterfactual statement, 'if a certain tumbler were struck in the right circumstances it would shatter'. 
My response is to further enquire what might be the truthmaker for this existent state of affairs. Place answers that, 'the state of affairs that makes the counterfactual true is simply the possession by the entity in question (the glass) of the dispositional property or passive causal power of being shattered when struck sufficiently hard' (1996c, p. 20). So, the truthmaker for the dispositional property is the existence of the counterfactual statement, whose truthmaker is the existence of a certain state of affairs such that an entity possesses the dispositional property in question. But this is the very property the ascription of whose existence we are attempting to guarantee. Place writes, 'Dispositional properties are modal properties, they consist in their possible future and past counterfactual manifestations' (Place, 1996b, p. 60). And, 'such dispositional properties are 'pure' in the sense that they do not consist in anything over and above a projection or orientation...of the property bearer towards what would happen, in the future [sic] certain conditions were to be fulfilled' (Place, 1996e, p. 119). Although the structure that causes the dispositional property presently exists, for the purposes of this debate concerning properties, the future has not yet arrived and the past counterfactual circumstances are not ontologically 'real'. ${ }^{24}$ Although Place does allow 'here-and-now' dispositional properties, he writes, 'but all that exists now is a state of the property bearer, a substantive law of its nature, which can be specified only by reference to its potential future manifestations' (Place, 1996e, p. 113). Thus, as Place acknowledges, the dispositional property does not exist in the 'now' in the sense in which the structure, itself, presently exists. If the dispositional property were reduced to its underlying structure, as Armstrong posits, then the warrant could be given in terms of the present existence of that structure. But since Place's dispositional properties are distinct from their causal basis (Place, 1996b, p. 63), no such solution is possible, and the present existence of the structure lends no credence to the existence of dispositional properties indexed to non-present circumstances.

\footnotetext{
${ }^{24}$ It is not within the scope of this Thesis to debate whether the future or past is fixed or open, although the impact of this topic on Place's dispositional properties would be an interesting future research project.
} 


\subsection{The Causal Role of the Microstructure}

A second objection to Conceptualism concerns Place's claim that the microstructure of an entity causes it to possess its dispositional properties. Place asserts that the state of affairs whereby an entity possesses a dispositional property and the state of affairs of the corresponding microstructure are distinct; and stand in a relation such that the microstructure causes the entity to possess its dispositional properties (1996c, p. 20). Yet he asserts that the microstructure is never purely categorical; rather, that dispositionality also features in it (Place, 1996e, p. 118).

Stephen Mumford argues that whether the microstructure were purely categorical or, as Place insists, partially dispositional (Place, 1996e, p. 109), the causal connection between the microstructure and the dispositional property would be problematic. In a case where the microstructure were purely categorical, Mumford notes that the categorical base would cause a disposition, which would cause the bearer to manifest power. However, since the microstructure is clearly capable of causation, why not suppose that the categorical base also causes the bearer to manifest power rather than, as Mumford writes, 'creating this separate dispositional property to do the job' (1998, p. 107)? If manifestation of power requires dispositions because the microstructure is somehow causally impotent, then how can the categorical base cause a disposition in the first place?

Moreover, given that the microstructure causes a disposition by virtue of its own partial dispositionality, this involves an infinite regress, since the categorical base cannot give rise to a disposition without first possessing some disposition to do so. Any such disposition must be caused by some other microstructure which, in turn, requires a still further level of microstructure, and so on. The result, Mumford concludes, is that possession of a disposition by some categorical structure requires an infinity of dispositions, suggesting that the microstructure cannot be causally connected to the disposition, as Place supposes (Mumford, 1998, p. 108). The regress is forced by the requirement that categorical properties cause dispositional ones, but that dispositional properties are required for such causing to occur (Place, 1996e, p. 119). There is no question of halting the regress by proposing irreducible fundamental dispositional properties, considering that Place's dispositions are caused by structural bases. 


\subsection{Summary and Conclusions}

This chapter has argued that Place's characterisation of dispositional properties, as consisting in nothing more than possible future and past counterfactual orientation of the property bearer, limits his ability to provide an adequate truthmaker for dispositional properties. Unlike Armstrong's categorical properties, whose existence is supervened upon the categorical microstructure of the property-bearer, Place's dispositional properties are distinct from any causal structural basis. Thus, the existence of the structure fails to contribute to shoring up ascriptions of dispositional properties indexed to non-present circumstances.

A second problem, described by Mumford, concerns a regress that arises because the structure of object-bearers is considered, by Place, to cause their dispositional properties. This structure is itself comprised of both categorical and dispositional components. Since dispositional properties are caused by underlying structure, each level of structure must contain dispositional properties, caused by still more basic structures and so on. This regress may only be halted by the existence of fundamental, irreducible dispositional properties. However, Place rules out this solution by denying the existence of dispositions apart from the presence of underlying structures that cause them to exist. 



\section{CHAPTER 10}

\section{ChaRlie MARTin \& John HeIL: Identity Theory of Properties - OUtline}

This chapter, and the following, outline and discuss the Identity Theory of Properties. Recognising the difficulties incurred by positing two distinct types of propertyqualitative and dispositional - at the fundamental level, Martin and Heil attempt to identify them (Heil, 2003a, pp. 111-112; 2005a, 2005b; Martin, 1993, 1996b, 1996c; 1997, p. 216; Martin \& Heil, 1999). Martin posited an earlier theory-known as the Limit or Dual-aspect Theory_-which he later clarified in terms of the Identity Theory of Properties (1993, 1996b, 1996c; 1997, p. 216; 1999). The Identity Theory employs the idea of fundamental 'power-qualities', which are at once qualitative and powerful (Heil, 2003a, 2005b; Martin, 1996c, p. 136; 1997).

The starting point for both these theorists is a denial that the dispositional and categorical are mutually exclusive. As Heil notes, if one sees the dispositional and qualitative as defined in opposition to each other, the idea of their being identical is unintelligible (2003a, pp.111-112). Hence, couching the identity in language that downplays the mutual exclusivity of the traditional dispositional and categorical dichotomy, the term 'categorical' is replaced with the term 'qualitative' (Heil, 2003a, pp. 111-112), specifying intrinsic, non-relational properties of objects. Like an ambiguous drawing (Martin, 1997, pp. 216-217) or a Necker Cube (Heil, 2003a, p. 120), the qualitative 'face' or 'side', respectively, provides the 'shell', and the dispositional 'face' or 'side' indicates what the bearers of these power-qualities 'do'. Importantly, whether any power-quality appears qualitative or dispositional will depend on how it is 'differently considered' (Heil, 2003a, p. 112).

Heil points to John Locke's account of qualitative but powerful properties as an exemplar. Locke writes:

Whatever the Mind perceives in itself or is the immediate object of Perception, Thought, or Understanding, that I call Idea; and the Power to produce any Idea in our mind I call Quality of the Subject in which the power is. Thus a Snow- 
ball having the power to produce in us the Ideas of White, Cold and Round, the Powers to produce those Ideas in us as they are in the snow-ball I call Qualities (1924: II. Ch. VIII, p. 66).

Locke famously distinguishes between primary properties, those revealed to us as they are in their objects, e.g. size and shape; and secondary properties (Locke's 'pure powers'), those that cause perception of certain properties that are not intrinsic to the object, e.g. colour (Heil, 2003a, pp. 79-82). Heil notes that all Locke's qualities are power-bestowing, and therefore the distinction between primary and secondary qualities should not be translated as a corresponding distinction between qualitative and dispositional properties (2003a, pp. 79-82). Hence, Heil collapses dispositional and qualitative properties together such that every property of a concrete spatiotemporal object is 'simultaneously qualitative and dispositional' (Heil, 2003a, pp. 11, 80-81; Martin \& Heil, 1999, p. 47). ${ }^{25}$ These constitute Heil's 'power-qualities' (2003a, p. 79); power or dispositionality ${ }^{26}$ is built into the universe and a property's dispositionality is strictly identical with its qualitativity, and both are strictly identical with the property itself (2003a, p. 111). The formulation is set out as follows:

If $\mathrm{P}$ is an intrinsic property of a concrete object, $\mathrm{P}$ is simultaneously dispositional and qualitative; P's dispositionality and qualitativity are not aspects of properties of $\mathrm{P}$; P's dispositionality, $\mathrm{P}_{\mathrm{d}}$, is $\mathrm{P}$ 's qualitativity, $\mathrm{P}_{\mathrm{q}}$, and each of these is $P: P_{d}=P_{q}=P(2003 a, p .111)$.

The strict identity entails that neither the purely qualitative nor the purely dispositional exists. Rather, these must be regarded as unrealisable limits of different ways of being a single property, leading to the stance that it is not possible to vary dispositionality without varying qualitativity and vice versa (2003a, p. 115). Moreover, properties are not comprised of separate dispositional and qualitative aspects, as the two cannot be somehow prised apart (pp. 111-112).

\footnotetext{
${ }^{25}$ The term 'higher-level' is described by Heil to mean 'a property possessed by an object in virtue of that object's possession of some distinct, lower-level realizing property' (p. $88 \mathrm{n}$ ).

${ }^{26}$ Heil sometimes uses these terms interchangeably (p. $76 \mathrm{n}$ ).
} 
The 'qualitative' is 'noun-ish' in that it describes states of affairs as they are arranged within any spatial region at some moment of time, e.g. 'shattered-ness when struck'. It is space-filling, involving definite spatial extension. Martin talks of it as that which is present in order for us to experience the world that we touch, feel and see. Importantly, it provides the 'what' whenever we encounter quantity:

The qualities of shape and size are intrinsic and provide the form and extent of the "shell" of the entities that have them. These qualities are space-filling...in that they are needed as intrinsic to what has size and shape and are to what the properties of size and shape are the extensional limits. They are needed for what is changing... When we consider the need for physical qualia (that is, qualities), even in the finest interstices of nature, largely unregarded and unknown, among them should belong the qualia (qualities) required for the sensing and feeling parts of physical nature (1997, pp. 222-223).

The ideas of 'extension through space' and 'space-filling' place the qualitative in the realm of the spatial. It is tied to the 'actual' in terms of a specific or definite spatial region at any given moment in time. But for both Martin and Heil, it also appears to be that which is 'intrinsic' in the sense of being non-relational.

When Martin talks of the dispositional, he has in mind capacities that, like the qualitative, actually and presently exist, whether or not they are exercised (1997, p. 202); they are 'readiness potential' for manifestation (1997, p. 210), and this 'potential' is ontologically robust. Unlike Place, whose dispositions are ontologically robust yet distinct from the categorical, Martin and Heil derive the robustness of dispositions by identifying them with the qualitative, such that the self-same property is both qualitative and dispositional.

Martin and Heil emphasises that the dispositional is definitely non-relational, reasoning that a dispositional property cannot be a relation to some manifestation (or possible manifestation), as that would conflate the two (2003a, pp. 81-83; 2007, p. 83; Martin, 1997). Martin notes, for example, that, 'dispositionality is not a relation between what is dispositional and what is its manifestation. It is not a relation because the dispositional can fully exist and be "ready to go" with the total non-existence of 
the manifestation' (1997, p. 216). Heil notes that, 'To regard dispositions as relations between the disposition itself (or some property grounding the disposition) and its actual or possible manifestations is to confuse a feature of our way of characterizing dispositions - conditionally by reference to their possible manifestations - for the dispositions themselves' (Heil, 2003a, p. 83). Martin and Heil separate out the disposition-existence from disposition-manifestation, which means that the disposition, in itself, does not consist of a relation. Rather, it is the manifestation of the disposition which involves a relation between the dispositional property and its reciprocal dispositional partners. Thus, for Martin and Heil, a disposition is nonrelational. In distinguishing dispositions or powers from their manifestations, Heil considers that a disposition is 'fully present in the object' and that 'relations come into the picture with manifestations' (2007, p. 83). The overall dispositionality-or 'dispositional profile' - of complex objects is the manifestation of the dispositions of its constituents (2007, pp. 83-84). Furthermore, dispositionality is to be understood causally, with cause and effect being explained in terms of 'Reciprocal Dispositional Partners'; whereby any disposition may have numerous reciprocal partners, in terms of which they have 'directedness' and 'selectivity' (Martin, 1997, p. 205). Martin uses the familiar examples of a lock and key as dispositional partners (1997, p. 204); or of water possessing a directedness and selectivity as a solvent for salt, but not for gold (1997, p. 205).

To compare the qualitative and the dispositional: The exhibitionism of the qualitative is intrinsically connected to the 'facilitative' forness of the dispositional. For Martin, 'What is exhibited in the qualitative informs and determines what is the forness of the dispositional, and what is the forness of the dispositional informs and determines what is exhibited in the qualitative' (1997, p. 216). Whereas the qualitative is 'noun-ish' in that it provides the 'what' for what it is that possesses properties; the dispositional is 'verb-ish' in that it depicts a process or potential process that, given an appropriate dispositional partner, arrives at some state of affairs, e.g. the disposition for (Martin's forness) 'shattering when struck'. Here are Martin's other characterisations of the qualitative and dispositional (1997, p. 216):

i) Both are equally basic and irreducible - no supervenience is involved. 
ii) Neither one can exist 'purely', i.e. they are like two sides of a Janus-faced coin such that neither exists without the other; any 'pure' conceptions of them represent unrealisable limits for different ways of being the same property.

iii) It is not the case that any property contains degrees of dispositionality or qualitativity ; that is, it is not the case of their being some combination of aspects of the qualitative and dispositional.

iv) They are not fully distinct or separable (although this is ambiguous in the body of Martin's writing, since he also claims them to be 'distinct').

v) What the property exhibits of its nature (the qualitative) equals that for which the property is directive and selective (dispositional) in terms of manifestations.

vi) They cannot be prised apart; each informs and determines the other.

vii) They are, in fact, identical with each other and with the property itself.

From this list of characteristics, two irreconcilable themes seem to emerge: First, The dispositional and qualitative are of two irreducible, but inseparable natures; and second, the dispositional and qualitative are of a single nature. These two themes are further outlined in what follows.

\subsection{Two Irreducible but Inseparable Natures}

Both Martin and Heil deny the existence of properties that are either purely dispositional or purely qualitative (see, Heil, 2003a, pp. 111-112; 2005a, pp. 352-353; Martin, 1996c, p. 133; 1997, p. 216; Martin \& Heil, 1999, pp. 47-48), which, as described in Chapters 3 and 4, leads them to criticise Shoemaker's causal theory of properties (Heil, 2003a, p. 76; 2006; Martin, 1997, pp. 213-215). The reasons, described by Heil, for denying the existence of purely non-qualitative worlds include that: First, relations need relata; second, we need to accommodate counterfactuality; and third, we must be able to distinguish non-qualitative objects from empty space.

First, relations need relata. Heil discusses world-models put forward by Richard Holton (1999) and Randall Dipert (1997, pp. 98-101) in which the world is a network of relations without relata, other than intersections of relations that form 
dense nodal points. Heil argues that relations need relata that are more than mere intersections of relations. (2003a, pp. 99-103). He argues that one reason why relata must be independent of their relations is to avoid a situation of interdependence: If relations did not exist except between relata that are only dense nodes of intersecting relations, then we readily end up with neither relations nor relata (2003a, p. 104).

Second, a world of pure powers would leave no room for the counterfactual nature of dispositions. Dispositional ascriptions are fundamentally modal but worlds composed of pure power must suppose necessary relations. A purely relational world has no room for modal truths, since it is composed of relations that are already actual. Put another way, if objects are nothing but their relations (including dense nodal intersections of relations), then the existence of an object ensures that the relations comprising it already exist. We get a fixed universe in the sense that it is not open to possibilities, thereby making it difficult for objects to possess dispositions in the first place (2003a, pp. 99-113).

Third, we cannot distinguish empty space from space with non-qualitative objects. Utilising an argument derived from an early Armstrong paper (1961), ${ }^{27}$ Heil, in concurrence with Martin, argues that properties such as shape, size, position, duration, divisibility, solidity and so on cannot, of themselves, give us a physical object because these properties could just as easily apply to any region of space (Heil, 2003a, p. 106; Martin, 1997, pp. 213-215). Even motion can be treated as a body 'occupying' adjacent spatial regions over successive intervals, and solidity as applying to one region of space being impenetrable to another. Therefore, something additional is required for distinguishing the presence of these properties from 'empty space'. This something is that which allows us the qualia whereby we see, hear, smell, touch and taste the objects of the world. Lacking this additional ingredient, we are left 'without a coherent conception of material bodies' (Heil, 2003a, p. 107), since a nonqualitative world would supply insufficient conceptual resources (p.100) to differentiate between space which is empty and space occupied by material objects. 'If an object's qualities are reduced to or replaced by pure powers,' writes Heil, 'anything resembling substantial nature fades away. Substances wholly bereft of

\footnotetext{
${ }^{27}$ Heil indicates that this is no longer Armstrong's view.
} 
qualities are difficult to envision' (p. 99). A non-qualitative world is, to all our sensibilities, 'empty of concrete objects' (pp. 76, 102).

In such a world, then, could objects be merely conglomerations of spatial points rather than substantial points? Drawing on an argument analogous to Richard Swinburne's (1980b) regress objection (1980a), Heil says no (2003a, p. 98). The world that lets us experience individuated objects as having shape, size, motion, solidity and so on incorporates either material objects or some 'field' of 'granular substance' (2003a, p. 101). This argument relies on the premise that even if properties like shape, position, duration, divisibility and solidity, of themselves, could be accounted for dispositionally, then the qualitative would still be required with respect to how these properties are detected. This criticism of pure-power worlds indicates that the dispositional lacks something which is provided by the qualitative.

As was shown in Chapters 2 and 3, Martin further criticises purely qualitative worlds on the basis that they lack modality (1993; 1996a pp. 127-129, 174-177; 1996b, 1996c). Heil (see, , p. 118) also argues against such worlds. His reasons include: First, that purely qualitative properties would not be detectable, and so we would never know of their existence. This claim takes us back to Locke's qualities whereby detection requires the ability to be detected - which is itself a power. We can never experience pure-qualities. Our experience extends only to qualities that are powerful. Why postulate entities that we can know nothing about?

Second, higher-level dispositions lack a causal role. Attempting to supervene dispositions on a purely qualitative ground encounters the problem of overdetermining the role of dispositions. If, as in Place's Conceptualism or Prior, Pargetter and Jackson's claim (1982, p. 255), the categorical base is the 'real' causal agent for an object's possession of power, then it seems that categorical properties are causally and irreducibly powerful, so any distinct higher-level dispositions are causally impotent. If the microstructure plays the causal role, there is no need to postulate dispositions over and above the microstructural base.

Third, an analysis of Categoricalism shows that strong causality requires irreducible dispositionality to be built into the system. Martin and Heil account for it by building it into properties. Thus irreducible dispositionality is built-in to properties, 
rendering them power-qualities (Martin, 1996c, p. 127). Here we have the converse of the above: the qualitative lacks something which is provided by the dispositional.

It can be nothing other than supposing that the dispositional and categorical each bring something unique to the world that fuels Martin's and Heil's criticism of monist stances that lack either the categorical or the dispositional. They assert that the qualitative and the dispositional are both required to fully characterise a property (Martin \& Heil, 1999, p. 47). This is particularly the case since the dispositional and qualitative are claimed to be equally basic, intrinsic, and irreducible to each other (Heil, 2005a; Martin, 1996c, pp. 132-133; 1997, p. 216; Martin \& Heil, 1999, p. 48). But the notion of 'reducibility', like 'supervenience', is tied closely to the idea of ascription. Is the irreducibility of the dispositional and qualitative, merely a matter of language, or do Martin and Heil have in mind an ontologically-robust distinction? The discussion leans toward indicating a robust distinctness, but the Identity Theory, as outlined in the next section, denies such a distinction.

\subsection{The Dispositional and Qualitative are a Single Nature}

The strict identity (Heil, 2003a, p. 111) of the dispositional and qualitative indicates that they are really a unitary 'one and the selfsame property' that cannot be prised apart. Martin notes, 'The only way that this can be expressed is by claiming that the qualitative and dispositional are identical with one another and with the unitary intrinsic property itself' (1997, p. 216). Properties do not combine dispositional aspects and qualitative aspects (1993, p. 184; 1996c, p. 132). Likewise, Heil warns that, "The identity theory is to be distinguished from theories according to which the dispositional and the qualitative are 'aspects', or 'sides', or higher-order properties of properties' (2003a, pp. 111-112).

Earlier, Martin explains that dispositionality and qualitativity are to a property in the same way that shape and size are to extension; they cannot exist without each other, although they can co-vary: 'The dispositionality and qualitativity of any intrinsic property is similar to the way shape and size are of extension. In each case, one cannot exist without the other, though one can vary without the other. Contra Hume and Armstrong, they are distinct but not separable' (1996c, pp. 133-134). Later, Martin clarifies the distinction as only abstract (1993, p. 184): 
What has been (misleadingly) called "The Limit View" (Martin 1994, 519) treats any intrinsic property not as a two-part compound of the purely qualitative and non-dispositional and of the purely non-qualitative and dispositional, but as a two-sided dispositional-qualitative coin. These cannot be abstracted as fully distinct and separable elements, but must be considered as unrealizable limits for different ways of being the same unitary property (1997, p. 216).

The 'abstraction' seems to indicate that the distinction is not ontologically robust; but merely the way that we conceive of it, such that in reality, there is only a single nature.

\subsection{Summary and Conclusion}

This chapter has described two prevalent themes that underpin the Identity Theory of Properties as put forward by both Martin and Heil. On one hand the dispositional and qualitative are both required, such that they each appear to make a unique contribution to the world. They are irreducible to each other, and as some have noted, have 'distinct natures' (Sparber, 2006). However, the claim for a strict identity between the two 'natures', and their collapsing into a single property—a power-quality—indicate that the distinction may be epistemological—how we 'consider' properties — rather than an ontologically-robust distinction. This is an important issue that is taken up in some detail in Chapter 11 to follow. A further topic discussed in Chapter 11 is Heil's claim that dispositional properties are non-relational. 



\section{CHAPTER 11}

\section{CHARLIE MARTIN \& JoHN HeIL: Identity Theory OF Properties - Discussion}

The Identity Theory rules out properties being either purely dispositional or purely qualitative, any combination of both dispositional and qualitative 'aspects', or some form of unity by which dispositionality and qualitativity can be reduced to each other. Invoking the term 'dispositional-cum-qualitative' as does Martin (1996c, p. 132), or the term 'powerful qualities' as does Heil (2005a, p. 352) in characterising properties, attempts to pick out the single nature of properties. However, both these labels are formulated by prior embedding in intractable terminology, combining concepts of the purely dispositional and the purely qualitative, which can only return us to the Limit View and thus to the dual-aspect nature of properties that both Martin and Heil are keen to avoid.

This chapter considers the Theory's ramifications, arguing that, based on the allowed parameters, the desired identity of the dispositional and the qualitative is not possible, and that the characterisation of a single-natured property may need to disengage altogether from the concepts of qualitativity and dispositionality in order to proceed on some other basis. Consider the following analogy in which nature is replaced by pigmentation. Let 'redness' represent the dispositional and 'blueness' represent the qualitative. Some purple objects of different hue appear 'red-ish' and others appear 'blue-ish'. Each object's purpleness is perhaps expressible as a degree of redness and blueness combined. However, Martin and Heil deny that the limits of spectrum - pure redness or pure blueness - exist. Correspondingly, they deny that the purple of any object can be any combination of degrees of pure red and pure blue. Accepting these restrictions, no object can be somehow 'red-cum-blue' or 'blue-red'. Similarly, we cannot describe properties as 'qualitative-cum-dispositional' or as 'power-qualities' while denying the existence of both the purely qualitative and purely dispositional. The upshot is that the restrictions laid down by Martin and Heil enforce the need to characterise the nature of properties without relying on the use of the terms 'dispositional' or 'qualitative'. (I describe an alternative basis in Chapters 13 
and 14 of this Thesis, arguing that the categorical-dispositional distinction has no meaning at fundamental levels).

In response, it might be argued that the analogy of pigmentation is not appropriate because a continuum per se is not apt for describing the Martin and Heil view. Identifying the qualitative and the dispositional removes the option of the two being distinct, and so being ultimate or pure ends of a continuum is rejected as a coherent analogy. The Identity Theory in this sense has superseded the earlier Limit View. But such a distinction is, indeed, embedded in the criticisms made of the competing monist theories. The possibility of two viable ways to consider a powerquality suggests that the dispositional and the qualitative appear as two different 'natures' (Sparber, 2006), each uniquely contributing to the world. The uniqueness is evidenced by the criticism that Martin and Heil make of the monist theories of Armstrong and Shoemaker - on the grounds that each fails to supply something crucial of the dispositional and qualitative respectively (Heil, 2003a, pp. 76, 111-112; 2005a; Martin, 1996c; 1997, pp. 213-216; Martin \& Heil, 1999, pp. 47-48). It would seem, then, that the two cannot be of the very same nature, particularly since the dispositional and qualitative are claimed to be equally basic, intrinsic, and irreducible to each other (Heil, 2005b; Martin, 1996c, pp. 132-133; Martin \& Heil, 1999, p. 48).

Their ostensible unique contribution pushes in the direction of robust distinctness between the dispositional and qualitative, but the Identity Theory denies such a distinction. The purported strict identity entails that the qualitative and dispositional are unitarily 'one and the selfsame property' - a 'power-quality' - and cannot be prised apart (Heil, 2003a, p. 111; Martin, 1997, p. 216). How does this fit with the seemingly unique nature of each? In particular, when we differentiate between the qualitative and dispositional in our consideration process, does this occur in virtue of any ontologically-robust feature of the property or its context; or do we make the distinction merely in virtue of how we perceive? That is, does our 'differently considering' power-qualities in terms of the qualitative or dispositional reduce to epistemology? In their discussion on picture theory (Heil [2003], [2007]; Martin [1997]), both Martin and Heil deny this claim, insisting that there is some truthmaker underlying the different ways we can consider properties. 


\subsection{Differently Considering the Dispositional and Qualitative}

It appears contradictory to suppose that the qualitative and the dispositional are each 'irreducible' and yet strictly identical, since the meaning of strict identity demands, surely, that identical things be reducible to each other. One way to avoid the contradiction would be to adopt an epistemological approach to their differentiation, explicitly allowing that: i) in terms of the way we talk about, or consider the dispositional and qualitative, the two are irreducible; but allow that ii) in ontologically-robust terms, the two are identical. According to this approach, when considering an object in terms of its behaviour, we talk about its dispositions; but in terms of how we sense it, we talk about its qualities. Our 'differently considering' an object's properties will sometimes lead to perceiving them dispositionally, and sometimes qualitatively, although the properties are the self-same properties across these differing considerations. Such an approach can be read into Martin's use of an ambiguous goblet/two-faces drawing:

What is qualitative and what is dispositional for any property is less like a two-sided coin or a Janus-faced figure than it is like an ambiguous drawing. A particular drawing, remaining unitary and unchanged, may be seen and considered one way as a goblet-drawing and differently considered, it is a twofaces-staring-at-one-another-drawing. The goblet and the faces are not distinguishable parts or components or even aspects of the drawing, although we can easily consider the one without considering, or even knowing of, the other. The goblet-drawing is identical with the two-faces drawing (1997, pp. 216-217).

Likewise for Heil's Necker cube example: 'The model, if you want one, is an ambiguous figure - a Necker cube, for instance - that can be seen now one way, now another' (2003a, p. 120). Construing the problem as a matter of epistemology would seem consistent with his view that, 'A property's dispositionality and its qualitativity are, as Locke might have put it, the selfsame property differently considered' (2003a, p. 112). In their discussion on picture theory both Martin and Heil deny this claim, insisting that some truthmaker underscores the different ways we can consider 
properties (Heil, 2003a; 2007, p. 82; 1997, p. 202). If this truthmaker is in the property itself, then clearly the qualitative and the dispositional each contribute uniquely to producing different effects in a perceiver, and this belies the claim that they are identical.

If not something in the property itself, can the truthmaker be found in the manifest world at large? Recall that the manifestation of the dispositions of objects occurs as relations between the numerous dispositional partners that constitute the physical context in which the object subsists. Since perception of an object is also a matter of its dispositions being manifested, Martin calls for the human mind as well as the physical environment to be considered reciprocal dispositional partners in the case of our perceiving the properties of objects (1997, p. 213). Merely epistemological differentiation in terms of our perception of properties has been denied. But could the physical environment, given that it works in conjunction with the human mind to bring about perception, be the relevant truthmaker for considering a property to be dispositional or qualitative in different contexts? This falls short of explaining why the self-same property would be considered in completely different ways - even given different physical contexts-unless the property has different features that could receive such different forms of focus. Martin and Heil rule this option out by denying dual aspects or any other combinatorial account of property-features (1999, p. 47).

Can differing functional roles, pace Stephen Mumford (1998), then, explain why we differentiate the dispositional and qualitative depending on how we consider the power-quality? Perhaps through reference to a property's functional roles, we appropriate one term or the other. Accordingly, whether considering a property to be qualitative or dispositional at any time might be tied to the function upon which we focus. If the focus is on, for example, how one property is individuated from another (e.g. sphericity from size), then the property would be viewed as qualitative. The same property might be seen as dispositional when the focus is on how it bestows power to its bearer (e.g. in virtue of being spherical, a ball can roll). Clearly, a single entity can play two roles, but in the sense that the Identity Theory of Properties posits only a single property types - power-qualities - it constitutes a property-monist position. This Property-monism, together with Martin and Heil's denial of two differing aspects to properties (1997, pp. 83-84), seems to rule out differing functions indexed to 
different property types. Alternatively, we might allow a property to be dispositional or qualitative, depending upon the function, by explicitly recognising that dispositionality and qualitativity each have unique contributions to make in terms of function. I have argued that this recognition drives the aforementioned criticism that both Heil and Martin make of Categoricalism and pure-power theories. 'To my mind', writes Heil, 'the identity theory is independently attractive, but, even if it were not, it appears to win by default! Purely qualitative properties lack appeal, as do pure powers. Mixing these does not help matters, nor does turning dispositionality and qualitativity into aspects or kinds of higher-order property' (Heil, 2003a, p. 120). If sustaining different functional roles, the contributions of the qualitative and dispositional appear unique, and this forces some justification of their purported strict identity.

A third scenario might be that varying functional roles are explicable in terms of differing physical circumstances or via differing epistemological considerations. However, both explanations having already been countered as suitable truthmakers for properties being differently considered without the difference being attributable to something in the property itself.

If something in the property itself is responsible for how a property is considered, then the dispositional and qualitative each contribute uniquely to producing different effects in a perceiver. Yet, if they are identical-if there is no ontological difference - then surely we have a monism. Talk of requiring both types of property seems redundant. Calling this ontologically-robust singular nature 'dispositional' or 'qualitative' or 'dispositional-cum-qualitative' is possibly inconsequential: It is ontologically singular regardless of the predicates used to describe it, the mode of perceiving it, or the functional role it plays.

\section{1..2 Non-relational Dispositional Properties}

Martin denies that dispositions need be considered relational, although he does postulate separation of dispositional properties from their manifestations; a stance that implies non-relational dispositions. Heil's stance is stronger in that he emphasises and explicitly defends dispositionality as non-relational. As Heil notes, 'There is, I believe, no compelling reason to regard dispositions...as relational. Dispositions can 
be conditionally characterized in a way that invokes their actual or possible manifestations. But this does not turn dispositions into relations' (2003a, p. 83).

Heil notes that critics of power theories may argue that because connections or relations have been re-located inside properties, rather than being external and existing contingently, every property must include all its possible relations (2003a, p. 123). Thus characterised, unmanifested dispositions would seem to push in a Meinongian direction because, they, in Ellis's terminology, 'stretch out' beyond the property bearer to the manifestation itself (2001b, p. 267), thus 'pointing' (Armstrong, 1997, p. 69) to possible manifestations that do not yet exist. This argument describes dispositionality in terms of unconstrained possibility. The Meinongian charge follows from the fact that possible manifestations have their subsistence in the disposition, and so if the disposition exists then the manifestation also has some kind of ghostly or pseudo-existence, by virtue of contributing to the identity of the disposition itself.

There is a built-in assumption that possible relations subsist in some ontologically-robust manner rather than as merely potential, if no categorical constraint or contingency is built-in to the system to make disposition-existence distinct from disposition-manifestation. As detailed in Chapters 2 and 3 of this Thesis, Armstrong's wedge is provided by the grounding of dispositional properties in categorical ones; and as discussed in Chapters 6 and 7, Ellis's wedge is provided by categorical dimensions. Heil and Martin's wedge results from denying that dispositions are relational, in the sense of stretching out beyond themselves to become continuous with their manifestation. By purporting dispositions to be intrinsic and non-relational Martin and Heil separate dispositions from their manifestations (2003a, p. $83 ; 2007$, p. 83), and thus avoid Meinongianism.

It can be argued that the distinction between dispositions and their manifestations applies to the Identity Theory of Properties both within and between objects. Heil notes that the dispositions of complex objects are derived by the manifestation of the dispositions of each constituent part. He writes that, "the overall dispositionalities of a complex object result from interactions among dispositions possessed by its constituents. Think of an object's dispositional profile as a manifestation of dispositions by its constituents' (Heil, 2007, p. 83). Were it the case that this dispositional profile entailed all dispositional properties of each of its 
constituent parts to all be constantly manifesting so as to produce that profile, then Meinongianism might be avoided, since all possible manifestations of the dispositional properties comprising the complex object are already manifesting. In this case, the step at which contingency would be required in order to avoid Meinongianism appears to be restricted to where objects manifest their dispositional profile in this way or that. And it is here that contingent circumstances seem to be required in order to account for the ways in which the profile is or is not being manifested. This is also where the notions of 'power-nets' and 'dispositional partners' (2003a, pp. 95, 97; 1993, p. 95) act to provide the contingency of the manifestations relations between objects.

Objects possessing a dispositional profile entailed by the dispositional properties of all its constituent parts might side-step Meinongianism as described above. However this would result in objects having their dispositional profile necessarily; given the composition of the object, one gets the dispositional profile. If all objects had such profiles necessarily, from where would the theory derive contingency of relations between objects, such that circumstances dictate how the profile manifests in any given situation? To provide for such circumstances, Martin and Heil build-in contingency to properties at the fundamental level, such that the properties of each constituent parts of each object is distinct from their manifestations. Hence the dispositional profile of objects must exist contingently rather than necessarily. Since each property is a power-quality, the contingency built-in to the system is given in terms of this singular dispositional-cum-qualitative nature of each property.

The non-relational status of dispositions raises the question of how Martin and Heil's power-qualities differ from Armstrongian-style categorical properties (properties I call 'pure-qualities'). As described already, the Identity Theory of Properties is arguably a property-monism, but with the pressing problem of how to characterise the singular nature of properties. Accepting the contributions of the qualitative and dispositional as unique gives rise to issues of their purported strict identity. However, positing their respective contributions as non-unique is also problematic, since it imposes the limitations of the qualitative upon the dispositional—since qualitative properties are intrinsic and non-relational, so too must 
be dispositional properties. Moreover, as discussed shortly, introducing non-relational dispositionality problematises power-qualities playing a dispositional role.

Dispositions that are self-contained and wholly-present in their instantiation, as are power-qualities, resemble Armstrongian categorical properties. Furthermore, just as those rely on contingent laws of nature for the manifestation of the power of their bearer, Martin and Heil's dispositional properties rely on contingent manifestation-relations, which arguably make dispositions distinct from their manifestations. Heil notes, 'Here we have relations, but the relations involve manifestations of dispositions. The resulting dispositions themselves are not relations - any more than the effect of a cause must, because caused, be a relation. Their existence, but not their identity, depends on relations they bear to other dispositions (Heil, 2007, p. 84). As discussed, this distinction applies within, as well as between, property bearers, since each constituent part of a complex object bears its dispositional properties, the manifestations of which contribute to the overall dispositional profile of the complex object (Heil, 2007, p. 84).

This Thesis has argued that Categoricalism fails to explain how a purely categorical world can provide the necessity required of strong causation. Although Martin and Heil are proponents of irreducible dispositionality, they remove the necessity between a property having an essential nature, and it manifesting that nature. By denying that dispositional properties are continuous with their manifestations, the Identity Theory of Properties is under a burden to explain how non-relational power-qualities differ from Armstrongian 'pure-qualities', since both appear to rely upon contingent relations extrinsic to the property itself for the manifestation of those dispositions. My claim is that necessity can only derive from relational dispositional properties, by which I mean, properties that give rise to necessary laws that describe the nature of those properties. That is, where relata are continuous with each other via relations, or, in Ellis words, dispositional properties that 'stretch out' (2001b, p. 267) rather than being self-contained.

Heil's defense of non-relational dispositionality draws on considerations of simplicity. Armstrong's pure-qualities require contingent laws of nature linking them. Together these categorical properties and laws would bestow power on the propertybearers. In contrast, Heil's power-qualities do not require contingent laws to bestow 
power, doing so through their own natures (2003a, p. 79) whose powers are 'built into' them (p. 124). As I have just argued, however, it is unclear how non-relational power-qualities do have their powers built-in if they rely upon manifestation relations that are distinct from the identity of the dispositional properties themselves.

Heil claims the ability for dispositions to bestow power is a brute fact: 'An identity theorist agrees that there is no further explanation for the fact that certain qualities endow their possessors with certain powers' (2003a, p. 117). He considers that this is no more mysterious than competing views, arguing that his position has the advantage over Categoricalism because his requires only a single brute fact - that 'power-qualities bestow power on their bearers'-whereas Armstrong presents both categorical properties and the laws of nature linking them, entailing at least two brute facts (p. 17). However, as Martin and Heil also require an additional manifestation relation to link dispositional properties with manifestations, it would appear that they also require two brute facts. I deem their case to be weaker than Categoricalism; given that they defend irreducible dispositionality, their Thesis lacks an explanation of how this cashes out in terms of power-qualities. If the difference between power-qualities and pure-qualities rests in the ability of power-qualities to bestow power without contingent laws of nature, then some detail of the action of 'bestowing' is required. Otherwise the theory presents essentially a deus ex machina leaving the notion of power-qualities incomprehensible. It appears that, as it stands, the relations involving manifestation are indistinguishable from Armstrong's contingent laws of nature.

\subsection{Summary and Conclusion}

I have argued that the two mutually-exclusive themes in the Martin-Heil Identity Thesis - the dual versus singular nature of properties - cannot be resolved either by proposing an epistemological explanation to account for different ways of perceiving properties, or by bolstering such a story with the addition of a physical context or differing functional roles a property might fulfill. If it is something in the property itself that is responsible for how the property is perceived, then it is hard to see how the dispositional and qualitative are a singular nature, since they each contribute uniquely to producing different effects in a perceiver. The uniqueness of the contribution is evidenced by the criticism that Martin and Heil make of the monist 
theories of Armstrong and Shoemaker-criticism on the grounds that each fails to supply something of the dispositional and qualitative respectively. Yet, Martin and Heil put forward strict identity of the dispositional and qualitative to form a powerquality, giving rise to the pressing problem of how to characterise such a property. The identity theory rules out either its being either purely dispositional or purely qualitative; or a combination of both dispositional and qualitative 'aspects'; or some form of unity by which dispositionality and qualitativity can be reduced to each other. This Chapter argues that, given these restrictions, describing the nature of this property will need to disengage with the concepts of dispositionality and qualitativity, and proceed on some other basis.

It was also argued that the non-relational status of Martin and Heil's dispositions renders their power-qualities indistinguishable from Armstrong's purequalities. First, if dispositions are non-relational, they supposedly differ from categorical properties in that, by virtue of their own nature, they bestow power upon their bearers. This is not explained, however, since they must be only contingently connected to manifestation-relations, yet rely upon these for the operation of power. Second, for properties to be irreducibly dispositional requires them to manifest in certain ways depending upon their essential nature. Separating the dispositional property from its manifesting relation, however, introduces an extrinsic, contingent factor into how it manifests. For power-qualities to differ significantly from purequalities, the irreducible dispositionality of the power-qualities must, of its own nature, be continuous with the manifestation relations, and in this sense, relational. However, according to the rationale of the Identity Theory of Properties, such entities are not viable, since, by strictly identifying the dispositional with the qualitative, the dispositional is constrained to a non-relational status. 


\section{SECTION 4:}

\section{FIELD-THEORY}

The conclusion to be drawn from sampling the Property Compatibilist positions presented in Section 3 is that accounts incorporating two completely different types of properties - or in Martin's and Heil's case, two different ways of considering properties - trip up at the point of showing why these two are required and how they interact. Categoricalism, outlined and discussed in Section 1, is a property monism claiming all true, ontologically-robust properties to be categorical. I have argued that it is unsuccessful in adequately accounting for the necessity required of strong causality. The monist position represented by the early Causal Theory of Properties, and discussed in Section 2, encounters a fork when attempting to resolve issues involved in reducing powers to properties and vice versa. The resolution requires either introducing quiddity or denying the existence of distinct entities.

The status of Shoemaker's spatiotemporal relations are implicated in the issue of distinctness between entities. Since all genuine properties are causally contributing, if Molnar's S-property argument holds, then these spatiotemporal relations should be seen as genuine properties, and by Shoemaker's criteria, intrinsic to their bearers. Indeed, if spatiotemporal relations were to be considered intrinsic, they would appear intrinsic to the system representing the inclusion of all of their relata. A related issue concerns whether the relata in question would be distinct, as Shoemaker proposes. It can be argued that whether the spatiotemporal relations are causally contributing or not, if the relata ground the relations as Shoemaker supposes, then the fact of distinct relata 'linking' together would have to be in terms of the properties of the relata themselves. Since the essential nature of power is that of 'effect', the self-containment characterising the individuation of relata would have to rely upon the quiddity of their properties. In early Shoemaker, quiddity is ruled out by virtue of the essential nature of all genuine properties being that which contributes to the powers of the things which possess them. As described in Chapter 5, a later reformulation of the Causal Theory of Properties introduces quiddity and hence fundamental categoricity into the ontology. 
The alternative course is to posit a Foundation-Monism allowing only noncategorical properties, and denying distinct objects, at fundamental levels. Section 4 explores this option by outlining and discussing a brief history of Dynamism as antecedent to more modern field theories, and then engaging with recent fieldtheoretic discussions and implications. Chapter 12 outlines the development of the early dynamic theories, and focuses on the Causal Theory of Harré and Madden, whose impact on my own philosophic stance has been considerable.

In Chapters 13 and 14, I defend a Foundational-Monist field theory that considers spacetime and the ostensible qualitativity of the manifest world to be emergent. The light-like network account that I put forward is influenced by the early dynamic theories of Roger Boscovich and Immanuel Kant, the philosophy of Rom Harré and W. H. Madden (the 'Great Field'); and indirectly by Keith Campbell's physicalised spacetime. ${ }^{28}$ The early higher-dimensional theories of Theodore Kaluza and Oscar Klein which pre-date contemporary string theory, and contemporary work in Quantum Gravity, has also been influential. Examples of philosophically interesting current physics research include Loop Quantum Gravity and the Helon Model. The Kaluza-Klein model is described in more detail in Chapter 14.

\footnotetext{
${ }^{28}$ While I do not adopt the fundamental categoricity that Campbell assumes, his discussion of Boscovich, Leibniz and other dynamic theorists seeded my own interest in field theories.
} 


\section{ChAPTER 12}

\section{FIELD-ThEORETIC VIEWS}

Thinking of fields as the ultimate fundamental entity goes back to the $16^{\text {th }}$ Century. Rom Harré provides an insightful, albeit brief, examination of the development of the Dynamical Theory of Physical Things, including Robert Norman's (1581) application of the tendency for magnetic orientation; William Gilbert's (1600) discovery of magnetism as the source of this, postulating structured 'spheres of power' around magnetic bodies (1975, pp. 164-165); Roger Boscovich's material points (1922); Immanuel Kant's fields of influence (1786/1909); Joseph Priestley's (1782) influence on Michael Faraday; James Clerk Maxwell's equations; and the subsequent development of contemporary quantum field theory (for detailed summary, see 1975, pp. 164-175). Keith Campbell (1976) also provides an insightful reading of Benedictus De Spinoza’s Monism (1632-1677); Gottfried Leibniz's Monadism (1969, 1996); and the material points of Boscovich (1922), whose spacetime Campbell 'physicalises'.

Harré narrates that although field theory has its roots in the $16^{\text {th }}$ century, it is not until the $18^{\text {th }}$ century that we get, from Robert Greene (1712), a clearly stated dynamic account of metaphysics. Greene held the view that material things have a range of dispositional properties (or powers), and that some of these produce experiences of what presents in Locke as secondary properties. In contrast to Locke's secondary properties, however, Greene's powers are all ontologically robust, not reducible to experienced properties of perception (1997, p. 21).

Later in the same century, Thomas Reid (1788) distinguished between passive and active dispositions, pre-empting Harré's distinction between dispositions as passive (liabilities) and powers as active (Harré, 1997, pp. 21-22; Harré \& Madden, 1975, pp. 86-87; Reid, 1788/1819). Around the same time, Immanuel Kant articulated that powers and only powers may be accounted as basic properties, and from which we may derive the matter of the manifest world. In his Metaphysical Foundations of Natural Science (1786), Kant outlines two fundamental forces-repulsion and 
attraction—with his Dynamism comprising eight propositions (1786/1909, Second Division, pp. 169-213 ):

i) Matter fills a space, not by its mere existence, but by a special moving force (p. 170);

ii) This special moving force is a repulsive force: matter fills its spaces by the repulsive forces of all its parts, i.e., by its own force of extension (p. 172);

iii) Matter can be compressed to infinity, but it can never be penetrated, by a matter (p. 174);

iv) Matter is divisible to infinity into parts, of which each is again matter (p. 176);

v) The possibility of matter requires a force of attraction, as its second essential fundamental force (p. 182);

vi) By mere attraction without repulsion, no matter would be possible (p. 185);

vii) The attraction essential to all matter is an immediate effect of it on other matter, through empty space (p. 191); and

viii) The original attractive force, on which the possibility of matter itself as such rests, extends itself directly throughout the universe to infinity, from every part of the same to every other part (p. 191).

Together, these propositions offer a comprehensive picture of matter, and as Kant notes, what we perceive as solidity is explicable in terms of forces:

If we review all [our] discussions on the above, we shall observe that the following things have been taken into consideration: Firstly, the real in space (otherwise called the solid) in its filling through the force of repulsion; Secondly, what, in respect of the first, as the proper object of our external perceptions, is NEGATIVE, namely, the force of attraction, by which, so far as may be, all space is penetrated, [or], in other words, the solid, is wholly abolished; Thirdly, the LIMITATION of the first force by the second, and the thence resulting determination of the degree of a filling of space; [we shall 
observe] therefore that the quality of matter has been thoroughly dealt with, under the heads of reality, negation, and limitation, in so far as they belong to a metaphysical dynamics (1786/1909, p. 199).

Kant's analysis concludes by supposing these forces to be primitive (1786/1909, pp. 204-205). By positing attraction and repulsion as fundamental forces, Kant explains, we arrive at our 'external perception' of the solidity of matter. Yet matter is fully accounted for by these forces.

Around two decades before Kant's exposition, Boscovich had proposed that everything is composed of material points, also acting upon each other according to forces of attraction and repulsion (1922). Keith Campbell describes Boscovich's system as a theory that 'bases itself on material points with no volume, no shape, no parts, and no mass, which act on one another at a distance in accordance with a single law of repulsion and attraction' (1976, pp. 86-87).

These early ontologies are surprisingly recognisable within the garb of modern physics, as can be appreciated by comparing Kant's and Boscovich's systems with Campbell's description of recent theories:

There are, however, modern speculations in which fields, like magnetic or gravitational fields, are the basic particulars. These unfamiliar entities extend indefinitely in all directions. They can interpenetrate. They have no surfaces and do not move. Our familiar material objects — which are solid, have definite surfaces, and are located at definite places - are the result of the interplay of fields, which gives a particular region different characteristics from its surroundings. Force fields have no size except that of the whole world, no shape except that of the whole world, no place except everywhere. Yet I suppose that they could be held to be spatiotemporal insofar as they fill and belong to spacetime. The essence of matter would thus involve just some kind of spatiotemporality (1976, p. 71). 


\subsection{Field-Theoretic View of Keith Campbell}

Campbell claims that the abandonment of atomism has involved a move away from thinking of particles as basic particulars and has required re-conceiving their boundaries as indistinct and overlapping (1976, p. 100). He describes spacetime as a totality in which there are no subdivisions or structures built up from a complex assemblage of parts (1990, p. 145). Unlike atomistic spacetime, described in terms of atoms and void, Campbell's physicalisation (or 'Geometrodynamisation') of spacetime describes it in terms of fields that are densities of mass/energy grounding the geometric feature of curvature (1976, p. 97). Imposing an identity between matter and spacetime, Campbell's matter is not superimposed upon spacetime, but rather, comprises, in the sense of consisting of or constituting, spacetime (1990, pp. 128129).

Some vagueness is introduced to the discussion if fields are considered to be both infinitesimally continuous and indefinitely extensive. (An in-depth consideration of quantised fields versus classical continuous fields is outside the scope of this Thesis, although will be briefly addressed in Chapter 14). Indefinite extension permits the overlapping and blurring of boundaries which gives rise to a picture of spacetime as holistic and monistic. As Campbell notes, 'this holistic, monistic, totalistic view echoes the conclusions of Spinoza. There is but one substance, and it embraces all reality; familiar bodies are but finite modes of extension' (1976, p. 104). A more detailed description of the field-theoretic view that Campbell envisions is provided in the following passage:

Suppose...we thought of the field as a real physical entity, with stresses and powers distributed through space, and the point as a mere mathematical abstraction. Then the point is a center of symmetry of the field. The values of the field build up symmetrically towards this center. The field associated with one 'point' has an effect on the field associated with each other point, to alter their centers of symmetry in regular ways. This is what we used to describe as the acceleration of one point particle by another. The interplay of these fields, which interpenetrate one another, gives rise to the observable phenomena of nature (1976, p. 99). 
While Campbell concludes in 1976 that the fundamental character of mass/energy may be considered in terms of 'physicalised' spacetime (1976, p. 101), in 1990 he canvasses doubts concerning the success of the Geometrodynamic program, put forward by physicists such as John Wheeler, whereby fundamental physical processes are accounted for through multi-dimensional geometry (Wheeler, 1962). Campbell notes that Geometrodynamics fails in attempting to identity matter and spacetime curvature (1990, p. 129), but favours a modified Geometrodynamics whereby mass/energy is antecedent to, or even produces, spatiotemporal curvature (1990, p. 129), echoing Harré's reservation about the ontic primacy of spacetime (1973, p. 223).

Campbell's physicalisation of spacetime incorporates the idea of structure and the field being ontologically-robust. In this sense he describes it as categorical. Marc Lange also provides an in-depth discussion on the 'reality' or physicalisation of fields - and hence their categoricity — arguing that such treatment solves the problem of action at a distance (2002). Graham Nerlich considers ontic originality in the curvature of spacetime, without committing to what this 'unique ontic category' might be:

[G]iving space a role in physical explanation need not, by itself, take us any nearer to showing that space may be understood as material when we treat it as real. It is always open to us to say that spacetime is a material field. Of course, the field can be regarded as material only in a somewhat attenuated sense and there can be little doubt that field theories have changed our concepts of the material and the physical. Hence, it is by no means clear that to describe spacetime as a material field accomplishes a material understanding of space and spacetime rather than a geometrical extension of the concept of matter (1993a, p. 129).

Both Harrè's Dynamism and Campbell's Foundationism (described in 5.2) attempt to explain how fields might account for the perception of solidity and shape. The big question is whether it is necessary to posit the categorical (or qualitative), or 
whether a pure powers account is defensible. If fields are something more than Boscovich's material points, but not categorical in nature, then a burden to explain this 'something' rests on the pure-power theorist. I argue in Chapter 14 that a characterisation of categoricity rightly includes that categorical properties as fully present in their instantiation and self-contained such that they accompany a notion of contingent laws. I also explain why I reject the view that 'categoricity' should be a term reserved for that which is 'actual' in the sense of being ontologically-robust; but that pure power might be considered to possess an ontologically-robust status although not categorical.

A concern that might be raised for the pure-power field-theorist is that of providing some categorical source of the field. However, assuming that power needs to be grounded in the categorical would be unsustainable, since the ability of such a source to give rise to the field would also need explaining and so on ad infinitum. This regress ends with only one or the other-power or categoricity-being primitive. Simon Blackburn argues that the requirement for a ground favours power theories rather than those positing Categorical grounds:

True, you might say, things like fields and masses are known by their effects on other things. But this should not prevent us thinking of them as in themselves categorical. There will be a categorical ground, G, for the (multitrack) disposition $\mathrm{D}$ whereby we know of mass or charge. It will be in virtue of the instancing of such a $G$ that an object has the mass that it does, or a region of space the charge...Presumably then there is a law whereby G supports D and this law imputes a power to G. So it ought to need a separate categorical ground, $G^{*}$, it being in virtue of $G^{*}$ that $G$ gives rise to D...But then the power of $G^{*}$, to bring it about that $G$ gives rise to $D$, will itself need a ground, and so forever. To stop the regress we need a brute or bare power without a categorical ground: better in that case not to insist on grounds in the first place (1990, pp. 63-64). 


\subsection{Harré and Madden’s Field Theory}

Building on a significant body of writing in the 1960s, Rom Harré published The Principles of Scientific Thinking in 1970 in which he focussed on the concepts of laws of nature, natural necessity, space, time and matter. Subsequent papers were devoted to the topic of powers and causal necessity, and saw the publication of Causal Powers by Harré and Edward H. Madden in 1975 and Philosophical Foundations of Quantum Theory by H. Brown and Harré in 1988. This section concentrates on Harré et al's Field Theory of Power described in the above and various other publications, setting aside the wealth of discussion on epistemological, linguistic and social themes in Harré's writing. ${ }^{29}$

The rationale behind Harré's Theory of Natural Necessity and his accompanying Dynamism is to overcome multiple problems persisting in the philosophy of science_-problems that, Harré proposes, are legacies of the 'Humean tradition', and which take the form of a group of claims or shared commitments regarding events as distinct, immediate and punctiform (Harré \& Madden, 1976, p. 95; Madden, 1972, p. 23). Harré et al. argue that certain of these doctrines, heretofore accepted as self-evident, can be refuted; and they attempt to do so through a field-theoretic view (Harré \& Madden, 1973, p. 209; Madden, 1973, p. 727). This attempt entails abandoning the notion of Humean-distinct individuals in favour of generalising the individual in terms of loci of force in what is ultimately an allencompassing field of potential; and thence to the ultimate or fundamental entities which correlate to 'centres of mutual influence' or 'singularities in the field' (1975, p. 161). The following account of Harré's theory begins with a discussion of the

\footnotetext{
${ }^{29}$ Space requires us to side-step most of the debate on Harré's Theory of Natural Necessity. Instead, I direct the reader to the extant literature, including discussions by Edward Madden and co-writers, Barry Cohen and Mendel Sachs, in defense of Harré's ontology (Cohen \& Madden, 1973; Madden, 1972, 1973; Madden \& Hare, 1971; Madden \& Sachs, 1972). I also refer the reader to Harré's and to Harré and Madden's publications setting out a defense of their theory of natural necessity (Harré, 1973; Harré, 1997, 2001; Harré \& Madden, 1973; Harré \& Madden, 1976; Madden \& Harré, 1973). For further reading of criticisms and responses, I recommend Henry Frankel's review of Harré's causal powers and Joseph Wayne Smith's response to Frankel (Frankel, 1976; Smith, 1982); Raymond Woller's criticism of Harré and Madden's account of causal necessity and Joseph Wayne Smith's response to Woller (Smith, 1984; Woller, 1982); Brian Carr's review (Carr, 1978); Edward MacKinnon's comments on Harré and Madden's 'Copernican Revolution' (MacKinnon, 1975); and in particular, Robert K. Shope's claimed correction of many of the errors made by these critics (Shope, 1988). Also of interest is David Miller's review of Harré's Principles of Scientific Thinking, and Barry Cohen's response (Cohen \& Madden, 1973; Miller, 1972).
} 
Humean event ontology, and then further discusses his 'Parmenidean Individuals', laws, and generative mechanisms.

\subsubsection{Events}

For David Hume, events are instantaneous impressions that are elementary and punctiform (Harré \& Madden, 1975, p. 110; Hume, 1739, Book I, Part III, Section XII). For Harré, however, events are not dimensionless points in space and time. Adhering to a 'common-sense' view of an 'event', he considers it to be something that happens to a particular across a period of time and a region of space. More specifically, they are enduring states of affairs. For Harré, a state of affairs is a persisting structure of a particular (e.g. a physical object), together with its properties and powers and relations between these. An event occurs when a state of affairs changes in some way or other (1975, p. 109).

If events are characterised, in Hume's manner, as immediate and punctiform, then their self-contained independence seems transparent. However, the ontological form does not necessarily reflect the epistemological one. Harré explores the Kantian principles of phenomenal and noumenal 'worlds', noting that while our phenomenal, manifest 'life world' supplies a reality incorporating substance and attributes, and is intrinsically bound up with consciousness, we cannot assume that perceived attributes pertain to the noumenal world (2004, p. 10). The role of an apparatus in the experimental framework is to 'force' the material world to interact with the apparatus, and so 'disclose itself in a perceptible form' (2004, p. 9). For example, a cloud chamber showing particle tracks forces the world to display itself in particle form (2004, p. 10). Each person, as a perceptual apparatus, is something like a scientific apparatus. We induce the noumenal world to manifest itself to us in particular ways, such as, for example, that of comprising substances with properties tied into a spatial and causal framework (2004, p. 11). Harré claims that the Humean construct of independence of events arises because of conflation of the epistemological with the ontological: 
Hume $^{30}$ argues that since we do have the idea of the action of causal power, and the connected idea of necessary connection between the nature of the powerful particular and what it can produce when it acts, and since reason alone could never give rise to it, we must find the original of this idea somewhere in experience. Then he proceeds to demonstrate in well-known ways that this concept could never be derived from single instances of any impression, either external sense impressions or internal impressions of the influence of the will. Hence we are never directly aware of the action of the causal powers of things. We come to the idea of causal power instead through the repeated experience of certain objects or events always occurring together. The firm habitual expectations established by such conjunctions are projected on to the external objects and events themselves and thus the concept of causal power is finally attained (1975, pp. 54-55).

Since our experienced sensations seem punctiform and distinct, it intuitively follows that this is how events are. And if events are distinct, then the notion of causality is derived as a constant conjunction of distinct events. Harré and Madden argue, however, that a distinction between the meaning of a proposition and the grounds we have for holding it should be observed. Failure to do so can result in questionable conclusions. Metaphysical atomism, for example, arose as a consequence of epistemic atomism, even though the latter does not entail the former (1973, pp. 120-123).

A consequent of the Humean distinctness of events is captured in the Principle of Independence (already discussed several times in this Thesis), which denies that there can be necessary relations between distinct entities (Armstrong, 2000, p. 8). Harré sets out the reasoning behind this Principle:

The heart of this argument, of course, and the backbone of the Humean tradition, is that there are no necessary connections between matters of fact. This contention is supported by the familiar Humean dialectic. If there were a

\footnotetext{
${ }^{30}$ This reference to Hume is with respect to his Treatise of Human Nature (Hume, 1962, part iii, sect. xiv) and Enquiry Concerning Human Understanding (Hume, 1993 [1740, 1777 post-humous edition], sect. vii).
} 
necessary connection of any kind between $\mathrm{C}$ and $\mathrm{E}$, then the conjunction of $\mathrm{C}$. $\sim \mathrm{E}$ would be self-contradictory. However, all events are complete in themselves and never alone require that any other event will or must result from them. It is very strange to think of water freezing when heated or air pressure decreasing with depth, but no matter how foreign these conceptions may seem there is nothing self-contradictory about them. Since the assertion of $\mathrm{C} \cdot \sim \mathrm{E}$ is never self-contradictory, it follows that there can be no necessary connection, logical or "causal", between them (1971, p. 12).

Given two wholly distinct entities, there is no contradiction in either existing without the other (Armstrong, 1997, p. 18). Likewise, if all events are independent or distinct, a conjunction between one occurring and one not occurring cannot entail a contradiction. Consequently, distinct events afford nothing logically or metaphysically contrary about nature's habits changing, and provide no explanation for any such change. For example, dynamite can suddenly not be explosive without any factor about the dynamite, other than its nature, changing. The argument can be set out as follows:

i) If there were a necessary connection between $\mathrm{C}$ and $\mathrm{E}$, then the conjunction $\mathrm{C} \cdot \sim \mathrm{E}$ would be self-contradictory

ii) If all events are independent, then the conjunction of $\mathrm{C} \cdot \mathrm{E}$ and $\mathrm{C} \cdot \sim \mathrm{E}$ is not self-contradictory

iii) All events are independent

Therefore

iv) There is no necessary connection between $\mathrm{C}$ and $\mathrm{E}$

The possibility for the inner nature of things to change without incurring contradiction is the basis for premise (ii) above (1975, p. 44). But, as Harré et al. claim, the phrase 'nature may change its course' has two possible interpretations: 1) that the nature of a particular remains the same even as its powers change; or 2) that a particular loses its previous characteristics (1976, p. 95). Madden and Harré argue that the first interpretation is self-inconsistent, and the second is irrelevant 
(1973, p. 120). We cannot use the nature of dynamite to explain its exploding while denying any connection between the nature of dynamite and exploding. Escaping this contradiction by claiming that the nature of dynamite might suddenly change-becoming, say, the nature of a stone - is an irrelevant prospect, since the nature of dynamite, not stone, is under discussion.

A possible reply on behalf of Humean ontology, pre-empted by Harré, is that the Regularity Thesis is not subject to the criticism Harré makes because a Humean would deny that the structure of dynamite (or stone) explains its explosiveness (or non-explosiveness). After all, the point of Humean regularity theory is that we can hope, at most, to recognise a constant conjunction between dynamite and exploding, so Harré's objection lapses within the domains of Humean ontology. While this effectively counters the contention of self-contradiction in Hume, it also renders the endeavours of scientific investigation impotent, as it undermines the utility of theories to explain and predict outcomes.

While this invocation of a disturbing explanatory lacuna does not make the Humean Thesis self-contradictory, as Harré et al. contend, even if change in the habits of nature were not self-contradictory, the Humean argument still fails. This is because it is impossible to demonstrate premise (i) without removing the Humean defense of premise (ii)+(iii). Premise (i) depends on $\mathrm{C}$ and $\mathrm{E}$ being conceptually independent of the natures of $\mathrm{C}$ and $\mathrm{E}$ - natures that would explain why $\mathrm{C}$ and $\mathrm{E}$ occur in succession (1973, p. 121). But such conceptual independence would entail that such natures do, in fact, exist in order to be independent. The thinking behind this claim is that the fact of $\mathrm{C}$ being $\mathrm{C}$ rather than $\mathrm{E}$, and vice versa, requires some differentiation between $\mathrm{C}$ and $\mathrm{E}$ other than their being simply numerically distinct. Numerical distinction is not equipped to sustain the qualitative distinction that is required to separate one event from the other in such a way that patterns of constant conjunction result. The argument is, thus, only against 'thin' events, and aligns with Harré's criticism of premise (ii), that it is inconsistent to propose that the nature of a thing both explains and does not explain its power.

A Humean may respond that, even admitting these natures, it is a further step to claim they explain powers - since they may be deemed categorical natures. However, in order that natures be natures, they must have some kind of 'ability', 
including, at the least, ability to be perceived. That is, they must provide for interaction, which is characteristic of power. Otherwise, they do no explanatory work, and then premise (i) is again jeopardised for exactly the same reasons given for nonexistence natures. The Principle of Independence requires something essential to events in order for the argument about the distinctness of events to even get off the ground; namely, there must be something ' $\mathrm{C}$-ish' about ' $\mathrm{C}$ ' and 'E-ish' about $\mathrm{E}$; an essential nature. Making this merely something akin to David Armstrong's individualising properties of thin particulars, there is no explanation why the constant conjunction of events should form various causal patterns by which we attribute causal interaction, as Hume claims of his Regularity Theory.

Armstrong keeps Humean Independence between his distinct, self-contained, categorical properties, but adds laws of nature as partial truthmakers for the dispositional attributes of things. His necessity relation, N, provides for lawful connection between types of properties. Thus, the laws, together with the repeatability of universals, allow predictability in the way properties connect and things relate. An essential nature is thereby provided, albeit attributed to higher-order types rather than property instances. In Chapters 2 and 3 of this Thesis, I argued that Categoricalism does not ultimately explain the necessity required of strong causality, something more than merely constant conjunctions between events. I also agreed with Charlie Martin's assessment that irreducible dispositionality is built into Categoricalism in the form of repeatability.

If Harré's argument against Hume's event ontology holds up, it would appear that even though Hume does not defend strong causality, he nonetheless requires events to possess some kind of qualifying nature in order to produce differences that give rise to patterns recognisable in constant conjunctions of events. That is, Hume requires event types entailing that events possess essential natures of some sort. Allowing for a purely qualitative nature, however, is not viable. Unlike Categoricalism, Humean event ontology cannot call upon necessity relations to provide type-ness, nor laws 'strong enough' to account for the diversification of conjunctions of events affording pattern-recognition. Since we could know nothing about events with a purely categorical nature, these would not suffice. 
If the Principle of Independence cannot be shored up, as Harré argues, how does this affect Shoemaker's Causal Theory of Properties and Place's Conceptualism? Chapters 4 and 5 discussed the Principle of Independence in relation to Shoemaker's mere-Cambridge, spatiotemporal relations. The problem faced by the early Causal Theory of Properties is how to explain why extrinsic relations should be deemed necessary. It was argued that because these relations are supervenient on the objects of the world, Shoemaker can provide for their necessity, but only at the cost of either ending with a Foundation-Monism, or by introducing quiddity into the theory and thereby rendering these relations extrinsic and contingently existing. The latter path was chosen for Shoemaker's later revision. Harré's argument against Hume's Principle of Independence is not an argument for extrinsic, contingent relations between distinct entities. Rather, it is an argument against the existence of distinct entities, and a bid for necessity to be built into the system by denying an ontology that relies on distinctness of events. Shoemaker's solution retains distinct objects at the cost of removing necessity from the system. Since his properties no longer feature irreducible dispositionality essentially - a property now maintains its identity throughout changes in its features - the causal features, although brute, become part the properties which possess them, apparently contingently.

Chapters 8 and 9 of this Thesis described how Place's Conceptualism encounters difficulties with the Principle of Independence, as it inserts a distinction between microstructure and dispositional properties in order to accommodate a causal relation between the two. However, a regress occurs because the fundamental structure, being comprised of both categoricity and dispositionality, requires a 'more fundamental' structure to cause it, and so on. This can be halted only by the existence of irreducible dispositional properties, not available to Place given that his dispositional properties are caused to exist. Harré's criticism against Hume's formulation of the Principle of Independence applies in full force to Place's Conceptualism, which bears the tension between requiring fundamental necessity and distinctness.

Given the assumptions built into Humean independence, necessity is unexplained in Humean terms, which make causality no more than an 'external relation between events' (Harré, 1970, pp. 104-105). If the external relation exists 
contingently, then there is no expectation of the regularities that we observe, and which need explaining. It can be argued that Humean distinctness underlies many other problems, including: denial of the uniformity of nature and hence inability to justify inductive inference; imputation of practices and assumptions behind scientific endeavours; and difficulty distinguishing causal from accidental sequences, scientific laws from enumerative generalisations, and subjunctive from indicative conditionals. However, Harré and Madden point out that such repercussions of the Independence Thesis are merely artefacts of the Humean ontology of events (1972, p. 24). 'These "problems", writes Madden, 'are not genuine ones but constitute reductios of the Humean view itself' (1972, p. 23).

One option, as Harré and Madden propose, is to deny Hume's concept of events as independent or distinct. 'Independence,' they write, '...is ontology-bound and not an intrinsic problem for every ontology' (1973, p. 727). If there were no necessary connections between matters of fact, then nature would have no obligation to proceed in the future as it has in the past, and we would lack any warrant to infer or confirm general laws on the grounds of instance-statistics. Independence, incurring theoretical implications that lead to explanatory paucity, is a major downfall of the Humean tradition, and alternative accounts must be explored. This is the departure point for Harré's Field Theory of Powers, and his positing ontologically-robust generative mechanisms underlying the strong causality upon which scientific endeavours rely.

\subsubsection{Laws of Nature and Generative Mechanisms; Distinguishing Causal from}

\section{Accidental Sequences}

In contrast to the Humean notion of laws - as generalisations from instance-statistics pertaining to constant conjunctions-Harré considers laws of nature to be descriptions of underlying mechanisms responsible for the constant conjunctions we observe (1970, p. 111). Without these mechanisms figuring in our laws, there is no distinction between those conjunctions considered causal, or nomic, and those considered accidental. An important principle in Harré's theory is to accept that necessity is tacitly built into our laws, and that we should be able to account for it. Furthermore, if our laws are statements about patterns, then we must be able to distinguish necessary 
from accidental regularities (1970, p. 99). According to Harré and Madden, the Humean tradition attempts to distinguish lawful versus accidental successions in terms of "nomic universals' by which one gets "the cash equivalent of "necessity" without any ontological mortgages':

Equally germane to our argument is the failure of any logicist doctrine adequately to characterise a law of nature and differentiate it from an accidental generalisation, a characterisation evidently necessary to any logicist account of causation. The contemporary Humean believes that there are 'nomic universals' which are more than mere generalisations over time, in terms of which a logicist account of causal statements can be given. He insists that such universals can be adequately analysed in logicist terms without requiring the concept of physical necessity. We believe not only that he does not sustain the claim, but that in principle such a claim cannot be sustained (1975, pp. 28-29).

Nomic universals are ostensibly true but also law-like, requiring that they share some formal characteristics. Examples are given by Harré and Madden, such as 'contain only purely qualitative predicates', and 'are unrestricted in scope'. Nomic universals give a sense of universality that is more than mere generality, but does not involve physical necessity. The problem is that it is unclear that we can derive general statements from purely formal or qualitative predicates, unconstrained by the inductive baggage of specific disciplines. 'But more frequently,' write Harré and Madden, 'the accolade is bestowed upon those general statements which have certain epistemic and logical relations to some corpus of scientific or common-sense knowledge and in particular are deducible from it' (1975, p. 29). A nomic universal must have a universal scope of predication, but such a thing calls for criteria to formulate and justify its category of restriction. Harré writes, 'The vacuous truth of an unrestricted universal is not sufficient for counting it as a law; it counts as a law only if there is a set of other assumed laws from which the universal is logically derivable' (1975, p. 31). Although these nomic universals differ from Armstrong's necessitating relation, 'N' (which does involve physical necessity), this critique is reminiscent of 
the difficulty faced by ' $\mathrm{N}$ ', since both nomic universals and ' $\mathrm{N}$ ' require higher and indeterminately-higher levels of law to underwrite the warrant for nomicity. But, as Harré and Madden note, this recipe fails to define 'lawlikeness', since other laws are needed to say what a certain law is, and these laws need still others, and so on (1975).

\subsubsection{Generative Mechanisms, Explanatory Regress and Parmenidean Individuals}

To give an adequate account of causality, the evidence for natural or strong necessity must be more than constant conjunction, for it must explain what underlies the successions from which we draw our inference statistics (Harré, 1970, p. 100). Whereas Hume's Regularity Theory of Causality denies access to the underlying structures of the world, Harré opts to consider generative mechanisms - more or less permanently existing mechanisms responsible for patterns among phenomena.

Harré's laws describe such generative mechanisms, which are comprised of inner constitutions, structures, powers, encompassing systems, and so on, and are responsible for the constant conjunctions between cause and effect (1970, pp. 104, 123). Positing laws as descriptive of underlying generative mechanisms is not to say we have access to knowledge of these generative mechanisms directly; or are somehow cognisant of the Kantian noumenal world. It is merely to assert that theoretically there is something underlying the patterns of behaviour and structure in the world. We look to the powers of things and patterns of effects in attempting to identify the generative mechanisms constituting the nature of things. This attempt follows the assumption of their existence. Based on the respective associations and patterns, we compose laws describing the powers - the capacities, liabilities and tendencies — of things (Harré, 1970, p. 123). Our law descriptions build up a model or 'hypothetical depiction of the nature of the actual world' (1970, p. 101). As noted by Harré, 'To say of a thing or material that it has a power to do, or to be, or to effect something or other is to say specifically what would happen under appropriate conditions, and to say that these effects occur in virtue of the nature it has, because it is to say only that it is in virtue of whatever nature it has that it can affect things, materials and observers the way that it does' (1970, p. 122).

At first glance, Harré and Hume appear not too different with respect to the composition of laws as descriptions of patterns or regularities. The difference rests in 
that Hume would deny the central feature of Harré's ontology, that things are to be identified and differentiated in terms of their powers; and that things possess powers in virtue of their inner nature (Harré \& Madden, 1975, pp. 5, 57). The strength of science lies in the predictive ability of models. Harré's stance is appealing because our overwhelming phenomenological experience of both necessity and possibility is acknowledge by fixing generative mechanisms into his system as an axiom.

If Harré's account is stronger than the Humean, its advantage lies in the model-making capacity that comes from axiomatically incorporating generative mechanisms. But its promise is fulfilled only if it accounts for our perceptions of necessity and possibility without a vicious explanatory regress. As Harré points out, there are regresses of micro-explanation and of macro-explanation. Regresses of micro-explanation occur when the underlying mechanisms cannot be observed but clearly differ in kind from our observations. Then we must hypothesise as to the nature of these mechanisms, using further models to do so, and still further models to explain these, and so on (1970, p. 261). This first kind of regress arises in trying to explain the mechanisms guiding the behaviour of things by concentrating on the relevant components and structure.

A second kind of regress, involving macro-explanation, occurs in attempting to delineate the structure of things with reference to the systems in which they are embedded; looking at wholes, of which the things under scrutiny are parts (1970, pp. 261-262). The macro-explanatory regress occurs because the complexity and scale of the structure that does the embedding might be indeterminate (1970, p. 262). There might, for example, be structure all the way down. A related regress, concerning cause and effect, runs in both directions (1970, p. 262).

Regresses can actually be useful for explanation, provided they are not ultimately vicious; and providing they are not infinite regresses. Harré approaches explanatory regresses by first delineating what needs explaining and what does not. He adopts the principle: enduring needs no explanation. What needs explaining is change, not 'unchange' (1970, p. 248). Harré's application of this principle derives from his rejection of Humean independence of events. In the Humean ontology, since all events are independent, potentially any event may follow any other. Thus, one following another on a regular basis calls for an explanation. As Harré notes, because 
things are either explicitly or tacitly treated as a set of 'like or identical events', in the Humean perspective, likeness or identity, and hence persisting or enduring, needs to be explained. The Problem of Induction arises, argues Harré, because the Humean requires an account of what remains unchanged:

The 'Problem of Induction' arises because it is supposed that we need an explanation of evidence for, or guarantee of sameness, of unchange. The problem is generated by asking 'What guarantees have we that the regularities and existences we have so far discovered will persist in the future?' It is then suggested that anything might succeed any previous state, and in the metaphysics of events (each independent of the previous one) this would indeed follow. But sequences of events are the products of generative mechanisms, regularities of appearances are the consequences of the endurance of internal structures. Persistence, endurance and unchange require no explanation and particular cases of them have no causes (1970, p. 248).

Because the regularities are brought about by generative mechanisms built-in to the universe, the existence of these generative mechanisms guarantee the persistence of the regularities. Since endurance requires no explanation, the enduring generative mechanism closes the explanatory gap for sequences of events. However, what explains the nature of generative mechanisms? Harré and Madden propose the existence of an ultimate entity that is unchanging (1970, p. 83; 1975, p. 163). Harré calls this a Parmenidean Individual, named in honour of the Parmenidean world-view, in which the essential nature of the world is eternal and unchanging. These entities are 'the rock-bottom referents of explanation', the ultimate explanans (1973, p. 223; 1975, pp. 162-165). An entity that can be identified by its primary qualities or properties is subject to change and cannot be fundamental. These changeable, material individuals, which Harré refers to as 'Strawsonian' (1970, p. 301) or Aristotelian individuals (1975, p. 96), have powers that are variable and therefore undergo change to which explanation applies. Parmenidean Individuals, although they constitute Strawsonian or Aristotelian Individuals, are bare power. Any nature apart from power, such as the Lockean primary properties of shape or volume, would be liable to 
undergo change. Harré and Madden describe a Parmenidean Individual as wholly characterised by its possibilities, incapable of being altered or transformed (1975, p. 163), and thus representing constant, rather than varying, power (1975, p. 96). Just as early Shoemaker's potentialities are immutable, but his individuals-ultimately comprised of sets of these potentialities - are subject to change under differing circumstances, so too Parmenidean individuals are changeless, although the 'objects' they comprise undergo transformations of properties and powers.

\subsubsection{Roger Boscovich - Law of Continuity}

Harré uses and extends the arguments of Roger Boscovich and Immanuel Kant in rejecting the 'classical atom' or 'corpuscle' (called an Aristotelian individual), as a candidate for the fundamental (1972, p. 155). Boscovich's discussion of the Law of Continuity, in particular, is crucial to Harré's characterisation of the Parmenidean individual. This Law denies that anything can pass from one magnitude to another without going through intermediate states (Boscovich, 1922, Article 18- 32, pp. 2527). What follows is an outline of the 'Boscovisch-Harré' lemma. ${ }^{31}$

Boscovich argues that if two solid bodies collide, then at the moment of contact each body must undergo instantaneous change of velocity:

[T]hen in every case it would be necessary that, at the very instant of time at which this contact happened, the hindermost body should diminish its velocity, \& the foremost body increase its velocity, in each case by a sudden change...For it cannot possibly happen that this kind of change is made by intermediate stages in some finite part, however small, of continuous time, whilst the bodies remain in contact (Boscovich, 1922, Article 18, pp. 24-25).

But an instantaneous 'jump' in velocity violates the Law of Continuity. To avoid this violation, one may either abandon the law (Boscovich, 1922, Article 30, p. 27), or take Boscovich's path in dispensing with immediate contact between solid bodies. 'But I, after considering the Law of Continuity somewhat more carefully,' writes

\footnotetext{
${ }^{31}$ For the details of this argument, I recommend the reader to pp. 285-293 of Harré's Principles of Scientific Thinking; and Boscovich's A Theory of Natural Philosophy, Articles 17 - 32, pp. 25-27.
} 
Boscovich, ' $\&$ [sic] pondering over the fundamental ideas on which it depends, came to the conclusion that it certainly could not be withdrawn altogether out of Nature. Hence, since it had to be retained, I came to the conclusion that immediate contact in the collision of solid bodies must be got rid of' (Boscovich, 1922, Article 31, p. 27). This could be done by positing, in line with Humean event ontology, a universe wholly disconnected among all its fundamental parts, an ontology that Harré rejects for reasons discussed in 12.3.1. Another way is to suppose that, at bottom, things are not corpuscular-not strictly particularised. Boscovich chooses this latter option.

Madden and Sachs observe that Boscovich's rejection of corpuscular ultimates is based on the supposed immutability of the Law of Continuity, which needs justification (1972, p. 155). Harré, recognising this, endeavours to extend Boscovich's defense of the Law:

For the general outlines of the proof of the Law of Continuity I follow Boscovich, supplementing his argument at some important points... Boscovich argues that two different kinds of proof ought to be offered. There should be an inductive proof, to show that the Principle in fact is operative in all known actions, but there should also be a metaphysical proof. This has the aim of showing that the Principle is necessary, in the sense that it is a principle which could not be abandoned without abandoning the whole scientific enterprise. Such a proof proceeds by showing how the Principle is related to very fundamental features of space and time. In his proof Boscovich simply assumes the continuity of time. I shall try to supplement his proof by advancing what I believe are powerful reasons for that assumption (1970, p. 287).

As Madden and Sachs note, Boscovich's metaphysical proof relies on showing that discontinuous action is not compatible with the concept of continuous time; but then it must be shown that time is necessarily continuous (1972, p. 155). Invoking Kant's kinematics, Harré first shows why it is impossible to empirically determine whether time is continuous or not. He then sets out a theoretical argument (Harré, 1970, pp. 289-291), summarised by Madden and Sachs as follows: 
His strategy is a reductio. If time is discontinuous, then motion is impossible. But clearly motion is possible since it exists. Hence time cannot be discontinuous. Harré establishes the crucial If-then premise...it depends upon the assumption that "a moving real particle in transition from place $s 1$ to place $s 2$ must necessarily be at every intermediate point in the course of the motion, and being a real particle must be capable, at least in principle, of being the subject of demonstrative reference at any one of those points (1972, p. 155).

Boscovich had assumed the continuity of time in his metaphysical argument for the Law of Continuity. But in trying to shore up Boscovich's proof of the Law, Harré incorporates it in his own premise. Boscovich-Harré together amounts to the continuity of time serving to support the Law of Continuity (Boscovich); but the Law of Continuity acts as a premise for the continuity of time (Harré). As Madden and Sachs point out, "the defence of the Law must be scrutinized to see that it contains no assumptions that beg the question at issue' (1972, p. 155).

To support this own field theory of powers, Harré depends upon Boscovich's refutation of fundamental corpuscular entities. However, Boscovich's unfounded reliance on the Law of Continuity is problematic. There are those in quantum theory, such as theorists of the Copenhagen Interpretation ${ }^{32}$, who might deny that the Law of Continuity is necessary or even viable. This leads Madden and Sachs to defend Harré's Parmenidean individuals by proposing independent criticisms of the Copenhagen view ${ }^{33}$. These include the inability of quantum theorists to describe what characteristics a fundamental entity might have. Quantum particles are, as Madden and Sachs note, 'extremely different from ordinary and classical particles - to the extent of not seeming to be particles at all-nevertheless, they are characterized in terms of some of the same primary properties of mass, weight, etc., as the classical atoms' (1972, p. 162). Quantum particles are treated in many ways like ordinary

\footnotetext{
32 Copenhagen Interpretation: At the quantum level, nature is essentially probabilistic. The Complementarity Principle is built-in to this interpretation, such that in any phenomenon, depending how one sets up an experiment, either the wave aspect or particle aspect can be observed, but not both simultaneously.

${ }^{33}$ Details of these criticisms are to be found in Madden \& Sachs, 1972, pp. 158-166.
} 
particles in terms of their mutability. It is not clear that Madden and Sachs succeed in discrediting the Copenhagen view, so the viability of the Law of Continuity remains in question. Moreover, recent theoretical questions on whether time is, indeed, continuous or discontinuous, negatively impact upon the transparency of the Law of Continuity.

Rather than wrestle with the Law of Continuity, perhaps Harré's reliance on it to support a field theoretic view is not necessary. In his discussion of Hume's event ontology, he supplied reasons to reject distinct events at fundamental levels, the default alternative is some kind of field-theoretic view. Whether in terms of classical continuous fields or a quantised field, there is currently considerable discussion in theoretical physics and in philosophy of physics concerning whether strict particlehood is any longer a useful concept, over and above its instrumental value. As discussed in 1.1 of this Thesis, Michael Redhead, Paul Teller and others claim that the notion of particle-hood is an outdated concept not supported by recent physics. Theories that are currently exploring quantum gravity, such as Loop Quantum Gravity (Smolin, 2000, pp. 106-145; 2006) and the Bilson-Thompson Helon Model (BilsonThompson, 2005; Bilson-Thompson et al., 2009; Bilson-Thompson et al., 2007), seem compatible with relational and purely powerful models of the universe.

Another concept of concern to Harré is that of the Parmenidean individual as an individual. If the ultimate entities are bare powers or potentialities, then how may they be characterised in terms of 'individuality'? Shoemaker faces the same concern, since bare powers cannot be atomic, thing-like entities - cannot be corpuscular or have qualitative features. The upshot is that Harré and Madden's Parmenidean Individuals require careful characterisation in terms of what it might mean for them to be individuals, as examined in the remainder of this chapter.

\subsubsection{Harré's Field Theory of Power}

Starting with the Parmenidean individual, Harré presents his conceptual system in six principles (1970, pp. 308-310):

Principle 1: The ultimate entities are quality-less point centres of mutual influence. 
Principle 2: The most fundamental material entities are atomic corpuscles, which are collections of ultimate entities.

Principle 3: Perceived things are groups of these corpuscles.

Principle 4: The mutual influences between the ultimate point centres must be at least attraction and repulsion, and depend on the distance between the centres.

Principle 5: The ultimates are individuated by verbal reference to their location in the spatio-temporal framework, but it is not an ontologicallyrobust, strict individuation in which these ultimates are somehow distinct from each other.

Principle 6: The ultimates are, themselves, neither perceptible nor detectable.

\section{Principles $1 \& 4$}

These describe the ultimate entities as quality-less point centres of bare power and mutual influence that are distributed in space. There is a constant flux of redistribution of these point centres and hence of their influences. Following Boscovich and Kant, the powers of these entities are the forces of attraction and repulsion ${ }^{34}$ at the very least (see, Principle 4), which may be viewed as fields of potential. A field, albeit with neither shape nor sharp boundary, has structure comprising the spatial distribution of point centres of gradients, or contours, of potential. The entities, then, are 'singularities' in fields of potential, whose essential natures are nothing more than powers. Harré defines potentials in terms of their effects, claiming that 'the potentials of the physicist's fields are its powers, that is the dispositions of the field in virtue of its having a certain nature at that point' (1973, p. 224).

To better understand these Parmenidean individuals, we may apply the metaphor of a landscape. We identify geographic places by their various featuresstructures involving the shape of the land rising or falling in relation to its surrounds. The 'fieldscape' can be envisaged as similarly 'structural' variations in magnitudes of potential. In close-up, distant mountain peaks turn out to be constituted of rocky

\footnotetext{
${ }^{34}$ In line with Boscovich and Kant, Harré seems to distinguish types of Parmenidean individuals in terms of attraction and repulsion, corresponding to primitive power.
} 
outcrops, each of which has 'peaks' and 'valleys' at successively smaller scales, until, somewhere below the atomic scale, changes in the potential gradient correspond to fundamental peaks and valleys. Pursuant to Boscovich and Kant regarding the degree of attraction or repulsion as contingent upon distance, we can see these 'maxima' and 'minima' as singularities - centres of influence whose magnitude varies by functions such as inverse-distance-squared.

\section{Principles 5 \& 6}

These points of mutual influence are not discretely distinguishable from the rest of the field. A theory of Parmenidean individuals within one universal field entails denying strict individuality for these singularities. Yet, as outlined in Principle 5, although they are not ontologically individual, they can be individuated verbally by reference to their location in the spatiotemporal framework. Harré's description of relativistic, non-absolute point-moments represents a system of location with respect to any arbitrarily specified frame of reference.

A substance-monist ${ }^{35}$ picture calls for some account of how we know the field exists at all, given that a single, indefinitely extended thing may apparently defy any frame of reference being established to characterise it. Can fish notice the existence of water? How do we perceive a universe of individual things, if in reality there is only one unified Great Field? According to Harré, because the field has structure as differentiations of potential, we may derive a concept of 'place' within it. The gradient of the field is continuously variable, allowing singularities to serve as reference points of their own distribution:

It is through the concept of a singularity within the field that field identification is possible. Singularities become reference points. By appointing [sic] to singularity the field of which it is a singularity is shown at least to exist. And since there is a conceptual connection between being that singularity and an element of that field, identification of singularities necessarily identifies fields (Harré \& Madden, 1973, p. 227).

\footnotetext{
${ }^{35}$ The term 'substance-monism' used with respect to Harré's 'Great Field' reflects a different context from that of the same term used in discussions on mind and body.
} 
Singularities can be considered loci of mutual influence or regions that are more dense or have greater potentialities in certain respects than surrounding regions. For example, a negative charge will repel another negative charge with indefinitely great force as a function of closeness, such that two singularities can never 'contact' each other. Described by Harré and Madden as 'structured elements of a unified and universal entity, the great field' (1973, p. 227), although such structure is a function of power, these elements appear to be localised in terms of spatial extension, yet persistent through time (1973, p. 228). This powerful structure dovetails with the suggestion made in 7.4 of this Thesis, that spacetime structure should be considered powerful rather than passive. It also raises the question of whether Harré's treatment of Parmenidean individuals is even-handed with respect to space and time, a discussion that is taken up in more detail in Chapter 13.

Although the singularities of this field are not strictly distinct from one another, they are distinguishable. In 1970 Harré postulates that the ultimate entities can be verbally, numerically individuated. He stresses that this is only instrumental, as implied by his principle 6 , whereby the ultimate entities are neither perceptible nor detectable per se, but we consider them in terms of individuation because we connect them with the ostensible individuality of manifest objects. If we are tempted to interpret Harré's 1970 'numerical individuation' of the ultimates in any more robust terms, Harré emphasises in 1975 that the potentials of the field, and their singularities or clusters of singularities, are not themselves spatially-individuated in the way that we perceive manifest objects as spatially distinct. We perceive, and conceive of, individuals; but, as described by Harré, a field-theoretic view posits merely potentials with loci structured in specific ways. 'But since there is no room for individuation', he writes, 'these acts [pointing to singularities] do not individuate fields, so we are free to interpret the singularities as being the structured elements of a unified and universal entity, the great field' (1973, p. 227).

Harré pre-empts the problem that, since he has used singularities to identify the field in terms of spatio-temporality, it would be circular to then use places in the field to individuate singularities. Instead, he describes the field as an 'ensemble of its potentials' such that 'places' can be understood in terms of comparative intensive 
magnitudes, rather than as spatiotemporally defined (1973, p. 229). Thus the field is described as an intensive rather than extensive distribution. (An example of an everyday intensive property is 'density'. An extensive one is 'volume'.) While the singularities constantly change their relative 'positions', assuming spacetime relationalism, they are not distinct entities. Each has a region of influence, but since this extends indefinitely (Harré \& Madden, 1975, p. 226), each region is co-extensive with the universal field. In this case, what we have taken to be Parmenidean individuals - singularities as centres of local influence - are revealed to be a unified, global entity. As Harré notes:

What would be the status of the field of a unified field theory, in which the universal field filled all space and all apparently ontically differentiated fields were somehow seen to merge within it? How could we have the concept of the field as an individual, an individual with powers, capable of taking on all the metaphysical roles so ill performed by the old concepts of the substance with its qualities? It seems clear that for the ultimate field the concept of 'individual' would have no application. Such a field could not be conceived of as an individual with powers (1973, p. 226).

An inability to individuate via spatial location is to be expected in an ontology in which there are only powers. The Parmenidean individuals of the Great Field are individuated only in terms of magnitude or intensity. While a field, itself, may be very structured-consisting of spatiotemporally distributed potentials - the elementary entities have no such structure in or of themselves. In this sense the Great Field is nothing over and above an array of gradients characterised purely by potentials (or pure powers) (1975, pp. 104-105). There is no question of the field as an individual possessing its powers; rather, the field is comprised of its powers. Harré's is, therefore, a bundle theory.

\section{Principles $2 \& 3$}

Abandoning independent singularities raises the question of how we perceive objects while supposing a substance-monist universe. It is important for Harré to integrate the 
scientific, monistic view of the Great Field and the manifest world of distinctly perceived objects. He asserts that our perceptions reflect the natural world in so far as the natural necessity that we perceive of things actually exists:

Our fundamental contention is that the necessity that is such a striking feature of the conceptual relation between the predicates descriptive of events, things and states of affairs as causes and the predicates descriptive of their usual effects, as it is unreflectingly understood, matches a natural necessity in the relation between the states, powers and natures of those physical systems which in fact constitute the universe...the conceptual necessity involved in relations between the predicates involved in causal hypotheses reflects the natural necessity of the upshot of the activities of physical systems (1975, pp. $8,10)$.

In positing 'natural necessity', Harré and Madden mean that if the constraining or stimulating effects of conditions are appropriate for the power of an object to manifest, then that power cannot but manifest (1975, p. 20). We ascribe power to things in judging what they may or may not do; but contra Hume's Regularity Thesis of Causality, we do so because we consider that things have a nature which underpins and explains our observations. Such 'causally active things', therefore, have their power not because of what they may or may not do. Rather, what they may or may not do-how they manifest their power-is a short-cut, counterfactual way of describing or speaking of the power of things. They actually have their power because of their intrinsic natures (1975, p. 86).

According to Harré et al., our intuiting natural necessity in the case of cause and effect occurs precisely because necessity exists in the natural world, and by this principle Harré defends strong causality. But this resurrects the question of how to account for the intuiting of particulars constituting the manifest world, if it is deemed to be substance-monist in nature? One answer lies in Harré et al's discussion of affordances: that perception or intuiting reflects nature is not to say that we perceive what exists exactly as it is. Harré's analysis of J. J. Gibson's affordances lends credence to this claim. Harré defines an affordance as a disposition 'the display of 
which occurs in circumstances created by or relevant to human interests' (Gibson, 1979; Harré, 1997, p. 25). We have our perceptual and conceptual apparati but, Harré argues, 'One must surely concede that no one system of instruments, the uses of which are made intelligible according to just one conceptual system, is going to give us a complete account of the physical universe' (1997, p. 26).

According to our perception, events involve objects, but we identify objects only because of the role that singularities play in relation to our apparati (Harré \& Madden, 1973, p. 228). Harré suggests that even though the world is monistic in nature, we perceive it as multi-faceted and individuated because of the structure of the Great Field. Harré denies Phenomenalism about the objects we perceive. He posits that fundamental material entities (atomic corpuscles) - as collections of ultimate entities - do indeed exist. Each cluster of ultimate entities is "jointly such that a continuous limiting surface of an infinite region of zero repulsive "force" surrounds it' (1970, p. 308). Within a structured region of the field, thereby differentiated from its surrounds, the bounds of material entities are just patterns of mutual effect of the ultimate entities of which that region is constructed. Taking, for example, the quality of solidity, Harré's claim - following Kant and Boscovich - is that what appears to be a primary quality may be accounted for in terms of forces of attraction and repulsion. The following passage provides some detail:

Solidity is the alleged quality, the possession of which is responsible for the fact that two material things cannot occupy the same place at the same time, and is logically connected with impenetrability, the power to resist penetration, in that the possession of the former is supposed to account for the manifestation of the latter. Solidity is supposed to be the permanent state of a thing which ensures that a thing has the secondary power to resist any other body. An apparently solid volume can be readily created around a centre of influence which affects other centres of influence with repulsive and attractive 'force'. The region around a centre of influence in which the repulsive 'force' is greater than the attractive will resist the penetration of other bodies. The surface around the point at which the net 'force' is zero will seem to be the 
surface of a compressible solid and outside that surface there will seem to be a free space in which these solid bodies attract one another (1970, pp. 305-306).

Harré provides further detail and similar treatments for the properties of inertia, density and so on. The upshot is a theory that explains the perceived properties of things, but dispenses with what we have previously taken to be qualitative 'substance' and its attributes at fundamental levels. Hence, although the world includes apparently individual, distinct objects, the ultimate entities composing such objects are neither discontinuous nor discrete.

\subsubsection{Questioning the Ontological Priority of Spacetime}

If a field is an array of potentials that are not intrinsically spatial, how can anything devoid of individual 'localities' be spatially distributed? The answer lies in recognising certain assumptions. If we think of spacetime as ontologically prior to, or background for, its 'contents', then it is natural to assume that the contents are spatiotemporally interactive or 'deterministic' in order that they possess mutually determinate spatial relations. But if, as Harré notes, fields are ontologically prior to spacetime, then the above issue need not arise. He questions the ontological priority of spacetime, as follows:

Fields are structures of spatially distributed potentials, enduring and changing with time. One is strongly inclined to reason a priori that from this characterization alone it follows that space and time are conceptually prior to fields, since these concepts are evidently required in order to provide even the most general characterization of the field. And yet it may turn out that in cosmology we may want to say that there is space and there is time only in so far as there is the universal field, that is that space and time exist only as relations amongst the states and component potentials of the field. It seems that while space and time are concepts necessary to the characterization of the concept of the field, the field is an entity necessary to the existence of space and time. Conceptual priority does not imply existential priority (1973, p. 223). 
We can construct a consistent picture in which the spatial distribution of potentials surrounding a singularity occurs precisely as a matter of continuous differentiation between their magnitudes, such that potential gradients vary according to a scale that emerges naturally. Then, the metric of space, say, just is the "well-ordering', ${ }^{36}$ of the measure of potential or power magnitude. We may use the attraction and repulsion forces postulated by Boscovich and Kant, for example, to see how the interplay of singularities in the Great Field determines distances between them. Consider a singularity whose power corresponds to some precise number of Parmenidean individuals. Given two such identical loci with mutual attraction and repulsion, were these forces wholly equal, there would be no possible net force. Moreover, if the two forces were to vary differently, albeit both according to inverse-distance-squared, then there would be just one critical 'separation' at which zero potential gradient obtains, thereby affording a metric based on nothing but numericity of Parmenidean Individuals. This picture does not construe distance to be primitive, assuming 'difference' in potentiality to be ontologically prior to spatial distribution. As alluded to earlier, the well-ordering of potentials represents a pattern of intensive, and not extensive, magnitudes, from which the relations of space and time emerge.

\subsubsection{Harré and Substance}

Instead of proposing a world of substance with attributes, Harré puts forward particulars as powers. Substance is traditionally that which persists throughout changes in its properties, from which it is distinct. In comparison, Harré's Aristotelian individuals persist likewise through changes in their powers, but are not strictly distinct from their powers, comprising regions of the field of power. This lack of distinctness is crucial with regard to what plays the role of substance in Harré's theory (a discussion addressed in more detail in Chapter 13 of this Thesis).

Let us consider the Parmenidean individual versus the Aristotelian individual. Parmenidean Individuals are singularities - regions in the field of potential whose magnitudes differ sharply from those of their surrounds, and-for the sake of sidestepping infinity - to indefinite limits. They are distinguished by a steep variation in

\footnotetext{
${ }^{36}$ A well-ordered set has a 'first' element and a specific sequential place for every other element.
} 
potential magnitude to the extent that Harré loosely calls them 'particulars'. Although I will argue differently in Chapter 14, an electron is one example of a fundamental entity, for Harré. Parmenidean Individuals do not persist through change, so given any change in its power, the electron would cease to exist. Since they are immutable, however, no such change is possible.

Harré's Aristotelian individuals, such as atoms, are clusters of Parmenidean individuals. The powers of an atom, unlike those of an electron, may change according to circumstance while its identity remains. Changes in its powers are credited to the interactions and reconfigurations of the Parmenidean Individuals, or singularities, of which it is comprised. However, just as with the Parmenidean Individual, we may readily consider gradients of potential surrounding the Aristotelian Individual, and these may be sufficiently abrupt such that it is distinguishable from other individuals in the field, yet not strictly self-contained. In this sense of being continuous with the Great Field, the traditional interpretation of 'substance' as self-contained and independent, does not fit with Harré's Aristotelian Individuals. Therefore, the traditional idea of substance as substrate to property cannot be accommodated in Harré's ontology. Instead, if substance is to be incorporated at all, then it is derived via pure power. Chapter 13 outlines one way such an account may be provided.

\subsection{Summary and Conclusions}

In summary, Harré and Madden's field theory of powers has much to offer in terms of explaining strong causation via enduring, underlying generative mechanisms ultimately comprised of a fundamental unchanging explanans - the Parmenidean individual-responsible for causal regularities. The primitive entity in this monist ontology is pure power; a dispersed and variously structured 'Great Field'. It is an intensive distribution of different magnitudes of potentiality, represented by overlapping contours and giving rise to the spacetime metric. The individuality of objects manifested to us is explained in terms of clusters of singularities whose patterned structures vary sharply from their surrounds. The qualitative properties of such objects emerge from the clustering of Parmenidean Individuals. 
Although unable to provide uncontroversial evidence for the Law of Continuity, Harré's model seems, relative to some competing ontologies, internally consistent and explanatorily powerful. His Great Field is a major influence in the formation of my own theory outlined and discussed in Chapter 14. 


\section{CHAPTER 13}

\section{The Substance Role in a Pure-Power World}

The thrust of the Swinburne regress and the neo-Swinburne arguments is that the categorical or qualitative is necessary to individuate the various properties attributed to things. The same contention applies to field-theoretic views as to ontologies comprised of distinct, fundamental objects. Martin argues, for example, that if the ultimacy of objects were replaced with fields, these fields would still be certain ways, and thus have properties that are over and above the field itself (Martin, 1997, pp. 222-223). For Martin, these properties need to be more than 'mathematized measures', by which he means they must be more than quantities or potentialities, that they be in some way qualitative (Martin \& Heil, 1998, p. 195). Putting forward his 'deeper' description of the world (Heil, 2003a, p. 189), Heil also explores the idea of a field cum spacetime manifold as the sole entity, whose manifest everyday objects represent properties, modes, or ways that this field is. For Heil it would have to be 'grainy', in keeping with Martin's view, requiring that the properties be somehow qualitative. Stances such as New Essentialism (2001b, 2002, 2008a, 2008b) include structure as fundamental while holding it to be the defining descriptor of the categorical. A similar view is also articulated in Mark Lange's (2002) and in Keith Campbell's (1976) 'physicalised' fields, each being categorical. Since pure-power theorists reject the requirement for power to be grounded in the categorical at fundamental levels, they face the challenge of explaining how the substance role is satisfied. This is the task of this Chapter.

The argument for fundamental categoricity goes hand in hand with the idea that power needs to be grounded by the categorical or qualitative. George Molnar discusses the notion of 'grounding power' as popularly understood in terms of bestowing power on its bearer (2003, p. 125), which can be achieved in two ways: either power is borne by some particular (or qualitative field) directly in virtue of its properties (Armstrong, 1997, pp. 69, 204-205; Martin, 1997, p. 197; Molnar, 2003, pp. 125-141); or dispositional properties are themselves grounded by categorical properties. I discuss the first of these in section 13.1 and the second in section 13.2. 


\subsection{Property Bearers}

The assumption that power needs to be borne and/or grounded is incompatible with fundamental power. This chapter will argue, however, that such an assumption is unnecessary; and makes the case for an emergent spacetime whose underlying structure can be considered powerful. The term 'emergent' is much debated in current literature. Some, such as Jeffrey Goldstein, use the term to refer to macro-level wholes that emerge from micro-level components (Goldstein, 1999, p. 50). My view, however, captures the notion of 'emergence' as increasing in complexity of structure from the micro-levels to the macro-levels, but sees the more fundamental levels as holistic. I use the term to depict a concept similar to the 'explicate order' put forward by David Bohm, whereby an underlying complex, dynamic and holistic fundamental level provides a principle of organisation that gives rise to the actualisation of higher order sub-wholes into relatively stable forms (Bohm, 1980, 1987).

Given the extent to which claims for categoricity permeate the literature, however, the onus is on the pure power theorist to argue for why such fundamental structure should not be deemed categorical, and to explain the manifestly qualitative world without recourse to fundamental categorical properties. To these ends, I suggest that the dispositional-categorical distinction, where this distinction is associated with the notion of possibility or contingency, obtains only at higher levels.

Both the Swinburne regress type objections (Armstrong, 1997; Armstrong, 2000, pp. 13-14; Armstrong, 2004b, pp. 138-139; Ellis, 2001b, 2002, 2005b, 2008b; Foster, 1982, pp. 66-72; Heil, 2003a; 2006, p. 42; Martin, 1993, 1996a, 1996b, 1996c) and the claim that fields must be categorical to have structure in some ontologicallyrobust way (Campbell, 1976; Lange, 2002; Martin, 1997, p. 195) insist that power requires a bearer, whether it be some haecceitistic particular or manifold. The underlying impetus for this assertion stems from the demand for some space-occupier or space-filler to be either the recipient or the antecedent of the action (or effect) of power. As Armstrong notes, it is objects or particulars that act and which are the recipients of action (1997, pp. 204-205). Descriptions of objects or fields as bearers of properties derives from Substance-Attribute Theory, in which substances are alleged to have attributes or properties that are distinguishable one from the other, and not identical with the bearer itself. Trying to explain how objects change, lose or gain 
properties over time is one cause for claiming properties to be possessed by, rather than identified (or partially identified) with, their bearers (Martin \& Heil, 1998, p. 198). Another reason for supposing that powers require bearers resides in the need to account for different properties in the same object, and different objects with exactly similar (in the case of Nominalism) or the same (in the case of Universalism) properties. The properties in question include those that bestow powers upon their possessors in one fashion or another.

The Substance-Attribute view, then, distinguishes between the powers of an object and the object itself. In his ontological argument, 'Five Ways', Thomas Aquinas puts forward an argument concerning change, which reflects why he favours this distinction. His argument begins by introducing potentiality and actuality as a dichotomy: 'For a thing only undergoes a change inasmuch as it has potentiality for being that into which it changes, while a thing only causes change inasmuch as it is actual' (Aquinas, 1270/1948, pp. 2576-2577). In modern parlance, potentiality is often associated with dispositionality, closely tied to the idea of 'possibility', and thus 'nonactual'. Actuality is often associated with categoricity or qualitativity . Hence, dispositionality tends to be connected with past or future, and categoricity with that which is present. (An example of this treatment was discussed in Chapters 8 and 9 of this Thesis, with respect to Ullin Place's Conceptualism.) Aquinas presents both the potentiality and the actuality of a thing as potencies: potentiality is its passive ability to undergo change, while actuality is its causing of change. Both are required for change to occur in the bearer of power. (This is reflected in the more recent work of Brian Ellis's New Essentialism, discussed in Chapters 6 and 7 of this Thesis, in which both causal powers and categorical dimensions play a causal role.) Aquinas also describes conditions required for the manifestation of power:

To cause change is just to draw something out of potentiality into actuality, and this can only be done by something that is in actuality. (Thus, something actually hot, like fire, makes wood which is potentially hot become actually hot, thereby changing and altering that wood.) But, while a single thing can simultaneously be in actuality with respect to one property and in potentiality with respect to another, it cannot simultaneously be in actuality and 
potentiality with respect to the one and the same property. (While that which is actually hot may simultaneously be potentially cold, that which is actually hot cannot simultaneously be potentially hot.) (Aquinas, 1270/1948, question 2, Article 3).

This passage suggests that no property of a thing can simultaneously be potential (dispositional) and actual (categorical). From this premise, together with the premise that both potentiality and actuality are required for change to occur, Aquinas implies that it is not possible for a thing undergoing change to be the cause of its own change in any particular respect. The argument can be set out as follows:

i) both the potential (dispositional) and actual (categorical) are required for change to occur in a thing's properties;

ii) the very same property cannot be both potential and actual (dispositional and categorical) in any thing;

iii) supposing actual property A were the cause of some distinct property B to change from potentiality to actuality, they could not belong to the same thing — since B would then be actual by virtue of property A; hence,

iv) power of a bearer cannot manifest — change cannot occur-without at least one object, external to that bearer, being involved.

The conclusion in (iv) implies that an object can effect change of a property in another object, but that an object cannot change the very same property in itself. An object, for example, cannot make itself warm if it is cold; another object, such as the external environment, is required.

If Aquinas's stricture is taken as a guide to understanding the Swinburne regress, then both potentiality and actuality are required for change to occur. Thus the manifestation or effect of power requires an ontologically-robust recipient bearer in addition to an agent of change. It follows that a bearer cannot be the recipient of the action of its own power. For an effect to occur, it must do so between distinct bearers of powers. (This principle applies to bearers considered at differing levels, for example, either within a complex object or among the mereological parts of such an object). The Swinburne regress, described in detail in Chapter 5 of this Thesis, alleges 
that if all of the essential, intrinsic properties of Shoemaker's bearers are nothing but powers (i.e. potentiality), then, because he lacks categoricity that is available for either potentiality to become or actuality to be, he cannot provide suitable recipients or agents for the manifestation of powers. Without bearers, the action of power is moot, since there is nothing for it to affect, and nothing to do the affecting.

Given this traditional background, power has been defined (in metaphysics) as the ability that a property bestows upon its bearer to affect or be affected (see, Armstrong, 1997, p. 69), which assumes that a bearer is indispensable for the existence and action of power. However, the requirement for property bearers, in the form of some instantiating particular, applies only to Substance-attribute Theories. No such requirement is feasible or necessary for Bundle Theories, whereby objects are comprised of bundles of properties, although Bundle Theories face problems of their own.

The first problem for Bundle Theory concerns the situation whereby an object and its complement of properties are identical, such that if an object loses or gains properties, it is strictly no longer the same object. The manifest persistence of objects that vary across time therefore requires explanation. An obvious response is that the objection is debatable, since discussion of an entity gaining or losing properties begs the question against Bundle Theory, which denies that entities consist of anything over and above their properties. A related objection can be put forward, though, querying how a bundle maintains its individuality through changes of composition. This may present a problem for Bundle Theorists purporting the existence of distinct objects, since they must explain why any given bundle is distinct from all others. However, the same concern may not apply to a theory that denies the existence of distinct objects or bundles.

A second, related problem for Bundle Theory is how to explain what ties the properties of any bundles together. However, this problem appears no more severe than issues that arise for Substance-Attribute Theorists concerning instantiation. As discussed in Chapter 3 of this Thesis, such theories need to address the instantiation tie (see, Armstrong, 1997, p. 114; Baxter, 2001) and Bradley's regress (Bradley, $1897^{`}$ ), topics under discussion in current philosophical debates. This issue is further discussed in section 13.3 . 
A third objection to Bundle Theory is encapsulated in Leibniz' Law, often referred to as the Principle of the Identity of Indiscernibles (PII). In his Discourse on Metaphysics, Leibniz writes, 'it is not true that two substances resemble each other entirely and are different in number alone' (Leibniz, [1686] 1973, Section 9). This Principle has often been read as meaning that no two entities can have exactly the same properties (Forrest, 2006), or that two entities with identical properties should be deemed the very same entity. A Bundle Theorist who believes that the world contains distinct objects might skirt this objection by counting numerical identity as a property. Thus, two numerically distinct objects can never have exactly the same set of properties. A Foundation-monist proposing a bundle view of the universe can respond to the criticism by pointing out that, since there is only a single object in the universe, PII does not have any threatening consequences. In such a view, bundles might be construed as regions of this field, whereby their locations are (higher-order) properties of the field. This position is further delineated in Chapter 14.

For a Bundle Theory that also disallows fundamental categorical properties, the meaning of the term 'power' could not be that of 'ability, bestowed upon a bearer by its properties, to affect or be affected'. If all the properties of an object were powers, which comprised objects, then 'power' would need to be defined, instead, as that which 'affects or is the recipient of effect simpliciter'. This raises the question of how, contra Aquinas, power can both affect itself and be affected by itself. Such enquiries, however, build-in an assumption of agent-hood, which pre-supposes distinct objects and causal effects in terms of relations between them. A Foundationmonist perspective, such as that being defended in this Chapter, holds no such assumption at fundamental levels. We may justifiably think of fundamental pure power in terms of an evolution of pure agency, reminiscent of Harrés primal 'Influence' (Harré \& Madden, 1975, p. 161). It is not until we consider the scale of higher-order, densely 'clumped' property-bundle configurations of field regions that 'objects' appear. It is these manifest objects that afford our impression of ostensibly 'distinct' agents affecting and being affected. These higher-order configurations may take the form of fermionic entities, as discussed in Chapter 14. 


\subsection{Categorical Grounding of Power}

The second assumption concerning power, that it requires grounding, derives from the claim that the individuation of powers or power-properties requires contingency, supplied by the self-contained nature of categorical properties. In a bundle theory of properties, the recipient might be the grounding categorical properties. Substanceattribute Theories may postulate categorical particulars bearing powers in addition to powers being grounded in categorical properties, or some other combination of provisions.

Molnar suggests four motivations for feeling that powers need grounds. The first two 'weak' motivations are: i) we feel the need to provide a truthmaker for powers that are unmanifested; and ii) assuming after Aquinas, that 'it is not possible that the same thing should be at once in actuality and potentiality in the same respect' (Aquinas, 1270/1948, pp. 2576-2577), we suppose actual existence and potential existence (i.e. unrealised capacity for existence) to be mutually exclusive. It would seem reasonable to require a ground for unrealised 'possibilities'.

John Heil is one proponent of these 'weak' reasons for grounding power. As he claims, dispositional ascriptions are fundamentally modal, but worlds composed of pure power must suppose necessary relations, and thus cannot account for a sense of possibility or the counterfactual nature of dispositions (Heil, 2003a, pp. 99-113). A tumbler possesses the disposition of brittleness because it will shatter when dropped in suitable circumstances, but this incorporates possibilities that need not be fulfilled. Since a purely relational world is comprised of relations that are already actual, it has no distinction between power and its manifestation, disallowing modal truths. Put another way, if we conceive of objects as nothing but their relations (or dense nodal intersections of relations), then the existence of an object ensures that the relations comprising it already exist. We get a static universe rather than one open to possibilities, making it difficult for objects to possess dispositions in the first place.

The purported distinction between power and its manifestation is based on assuming that: a) certain powers of an object might never be manifested; and b) that when an object does manifest power, it does so according to variable and contingent circumstances. If an object's powers and their manifestations were one and the same, there would be a world of strictly necessary laws of nature in which circumstances 
could neither be, nor have been, otherwise than as they transpire. Such a world has no room for ontological possibility (Heil, 2003a, pp. 99-104). In this light, it has been argued that the reservation of ontologically robust status to the categorical is motivated by the perceived need to accommodate contingency and to partition power from its manifestation. As Armstrong notes, the proposition that power needs to be grounded in categorical properties goes along with upholding contingent laws of nature (Armstrong, 1997, p. 83).

There is something interesting about the idea that categorical properties provide for contingency, and thus possibility. Yet, in many descriptions of dispositional properties, it is these that are described in terms of potentiality, possibility and capacity, and hence their being 'non-actual'. I will return to this point in Chapter 14, where I will argue that a 'purely powerful world' need not be taken to mean the same thing as a 'purely dispositional world', if the term 'dispositional' is employed to refer to possibilities and hence associated with being 'non-actual' or 'actual but nonmanifesting'.

Denial of categoricity at fundamental levels does appear consistent with necessary laws, and such a view seems committed to the reduction of possibility to the epistemological. Beyond this 'cost', however, there may be no need to accommodate contingency, nor allow for unmanifested powers, at fundamental levels, although we may provide for epistemic possibility. Taking a 4-dimensional block universe (4-D) perspective, the intersections of object world-lines represent interaction. At any given time slice, the possibility of two world-lines intersecting is defined by the conjunction of their respective 'future light cones'. The extent to which light cones overlap pertains to the distribution of mass-energy associated with power or potentiality. However, the notion of 'possibility' embedded within our use of counterfactuals is pertinent because we are blind to the future. We do not possess a 'God's-eye point of view' to know 'the end from the beginning' (see: Isaiah 46:10). Observing whether any two world-lines actually intersect, God has no use for possibilities. In a 4-D world-model, possibility arises due to the inability to see time slices 'ahead'. In a purely relational universe, possibility arises similarly, due to the inability to see beyond a certain radius within the relational net. In either case, it is an epistemological abstraction. 
Considerations of whether the universe is fundamentally indeterministic, with ontological possibility built-in as randomness, is under discussion in physics at present. Issues include whether the probability that features in Quantum Mechanics is subjective or objective, and whether measurement entails irreducible uncertainty (see, Caves et al., 2008). Regardless of these debates, the appearance of possibility can be linked to the emergence of higher-order categorical properties at levels where distinctly persistent, non-homogeneous clumpings of mass-energy become noticeable; since one of the prominent features of categorical properties is their ascribed contingency in terms of individuating properties and objects. As argued later in this chapter, such manifest distinctness may feature at higher levels yet remain absent at fundamental levels. Indeed, the dispositional-categorical distinction can have meaning only at levels that feature such apparent categoricity. A pure-power Foundationmonist ontology, discussed further in Chapter 14 of this Thesis, may obviate the categorical-dispositional dichotomy at fundamental levels, while explaining why it appears as higher-order phenomena.

Two 'stronger' motivations suggested by Molnar for grounding powers in the categorical are that: iii) we need to explain powers, and since there seem to be far fewer prospective grounding candidates among categorical properties than among powers presenting themselves, we can more readily get an explanation in terms of non-power properties than of powers; and iv) many powers, particularly those of complex objects, appear to have a known base; and accordingly we can extrapolate and suggest that all powers have bases (Molnar, 2003, pp. 126-127).

In accord with a solution that I defend, Molnar proposes that a suitable response to the grounding issue might be to 'allow ungrounded powers full ontological status on par with all of the paradigms of respectable existences' (Molnar, 2003, p. 141). But this requires powers to be more than mere possibilia, since it involves actuality in the sense of ontological-robustness. Power, thus conceived, usurps this traditional mark of the categorical. (See the discussion on Conceptualism in Chapters 8 and 9 of this Thesis.) It also challenges the understanding of power in purely dispositional terms, if the term 'dispositional' is taken to denote potentiality or possibility. Molnar warns that this step brings with it a host of problems, but opposing 
theories also face their share of difficulties, many of which have been discussed in this Thesis in considerable detail.

Swinburne regress arguments posit the intuitively satisfying position that we cannot 'see' the effect of power, or see properties, unless these are grounded in the categorical. As also described by Harré's and Madden's critique, Simon Blackburn discusses the role that Humean event ontology plays in our ostensible need to ground powers in the categorical. He writes:

In Lewis's exploration of these issues ${ }^{37}$, categoricity comes in the 'Humean mosaic' or pattern of 'perfectly natural intrinsic properties which need nothing bigger than a point at which to be instantiated. It seems as though we need them, but it now also seems as though we cannot have them - out [sic] best physical understanding of the world gives us no conception of what they might be. But the mention of Hume is suggestive. We can think of the Humean mosaic in experimental terms: a colour here, a tactile sensation there, a sound somewhere else. Categoricity in fact comes with the subjective view: there is nothing dispositional, to the subject, in the onset of a pain or a flash in the visual field. Such events come displayed to us as bare, monadic, changes in particular elements of experience. In this perspective a change in perceived colour is as categorical as a change in shape or a twinge of toothache, even if from the objective standpoint, 'all that goes on' when such changes occur is that a change of foundational (dispositional) state arises, the subject being disposed to act and think differently as a consequence of changes in the dispositions of surroundings things (1990, p. 65).

This passage explains that we intuitively perceive not possibilities, but what seem to be actual events. Events that affect us via our modalities inform us of the impact 'concretely', as a change in 'substance' rather than potentialities. The powers that manifest as effect thus seem grounded in categorical antecedents, and we feel there must be a 'what' that we perceive, not simply that we are 'caused' to perceive. Anjan

\footnotetext{
${ }^{37}$ Blackburn takes his reference from Lewis, 1986, p. x.
} 
Chakravartty argues, however, that empirically verifying any such 'what' is not possible:

One might contend that on the assumption that there is more to property identity than the dispositions for causal behaviour that properties confer, or rather more specifically, if we were to say that we can recognize properties independently of causal relations, the regress could be broken. It seems impossible, however, to give any empirical content to this suggestion. If there were something more to causal property identity than dispositions conferred say, as per categoricalism or double-aspect theory - how would we recognize the extra somethings? It would appear that we have no option but to ground property attributions on causal interactions that we experience or detect (Chakravartty, 2003 , p. 397).

Although we cannot directly encounter the 'what' of perception other than through a 'perceptual veil', it is considered by proponents of the Swinburne regress that the contradictory view - that perceiving is no more than responding to causal effects, and itself is but a link in the causal chain - is anti-intuitive compared to the argument for Kantian noumenal objects. The idea that 'real' percepts are not 'things' per se, but rather, parts of causal chains in which effects are passed on, unacceptably discomforting to many.

As briefly described in Chapter 10, Heil points out two further facets of antiintuitiveness: first, relations need relata; and second, without the qualitative, we could not distinguish empty space from space containing non-qualitative entities. Concerning the first idea, Heil discusses world-models put forward by Richard Holton (1999) and Randall Dipert (1997, pp. 98-101) which have networks of relations without relata other than intersection points with different densities of relations. These models might be used to represent spacetime, for instance, as comprising the network links, whose 'contents' are defined by the nodes corresponding to Harré's Parmenidean individuals or field singularities, perhaps. In this case, the network density reflects the embedded geometry or curvature, which is inseparable from the contents. Such worlds need not incorporate spacetime points as existing in any 
substantial sense. Rather, the 'locations' of individual nodes are characterised with respect to their arrangement of links. ${ }^{38}$

Heil asserts that relations need relata, and denies that these can be merely dense nodal intersections that are characterised entirely in terms of their relations to other such nodes (2003a, pp. 99-103). One reason he gives, for why relata must be independent of their relations, is the need to avoid a situation of interdependence: if relations did not exist except between relata that were only nodes of intersection, then we would readily end up with neither relations nor relata (2003a, p. 104). For Heil, the nodes serve to link up the links. As I see it, however, this problem occurs only for a world that has no relations in the first place. In a proposed purely relational world, relations can be thought of as fundamental and existing in and of themselves over and above being merely abstracted associations of relata. Armstrong's description of relations as poly-adic properties comes to mind (Armstrong, 1997, pp. 85-87). They do exist, and intersections among them correspondingly exist.

Heil's second argument (2003a, p. 106), for why the notion of a pure-power world is anti-intuitive, derives from an observation made by David Armstrong (1961). ${ }^{39}$ As noted in Chapter 11, Heil argues that properties such as shape, size, position, duration, divisibility, solidity and so on cannot, of themselves, give us a physical object because these properties could just as easily apply to any region of space. Even motion can be treated as a body 'occupying' adjacent spatial regions over successive intervals, and solidity as referring to one region of space being impenetrable to another. He concludes that something additional is required for distinguishing the presence of these properties from 'empty space'. This something is what allows us to sense - to see, hear, smell, touch and taste - the objects of the world (2003a, p. 107), and that a non-qualitative world would not supply the conceptual resources (2003a, p. 100) required to differentiate between space which is empty and space occupied by material objects. 'If an object's qualities are reduced to or replaced by pure powers,' writes Heil, 'anything resembling substantial nature fades away. Substances wholly bereft of qualities are difficult to envision' (2003a, p. 99). A non-

\footnotetext{
${ }^{38}$ The topic of ontic structuralism, although outside the scope of this Thesis, is relevant to this discussion.

${ }^{39}$ Heil is careful to make clear that this is no longer Armstrong's view.
} 
qualitative world is, to all our sensibilities, 'empty of concrete objects' (2003a, pp. 76, 102).

In such a world, then, could objects be merely conglomerations of spatial points rather than substantial points? Drawing on an argument analogous to Richard Swinburne's regress (1980b), Heil says no (2003a, p. 98). The world that lets us experience individuated objects via shape, size, solidity and so on incorporates either materiality or some 'field' of 'granular substance'. This conclusion relies on the premise that even if properties like shape, position, duration, divisibility and solidity of themselves could be accounted for dispositionally, then the qualitative would still be required with respect to how these properties are detected. A related viewpoint is that, in a world of pure powers, qualities would be needed in order to differentiate the effects of powers. Otherwise we lack an explanation for how objects and properties are distinguishable from one another, given that pure power does not, of itself, seem to provide for objects or properties to be individuated. Heil focuses on the individuation of powers when he writes that, 'qualities inescapably enter into the individuation of powers, and in a way that makes it hard to see how these could vary independently' (personal communication, 2007, August 8). Martin expresses a similar thought, but focuses on objects, by noting that qualities supply the forms of their possessors (Martin, 1997, pp. 222-223). As Blackburn also says, the intuitiveness of requiring a 'what' of perception is derived from our being accustomed to regarding the world in terms of distinct objects, perceived either directly or indirectly. As discussed further in Chapter 14, I argue that a Foundational-Monist position denies that distinct objects exist, or that power is 'particularised', at the fundamental level, and therefore does not fall under the regress objection.

There is a further reason for denying the force of the regress argument. Blackburn suggests that to avoid the dilemma of choosing between what is in keeping with physics but anti-intuitive and what is intuitive but not physically possible, we tend to avoid looking too closely:

Strawson counsels that we can self-consciously shift perspectives to avoid the contradiction of thinking of the same event as both categorical and dispositional. But the problem remains that this gives us no help in 
understanding what, except counterfactuals, is true of the objective order of nature, unless, heroically, we see that order as a kind of construct from the categorical point-instances of properties available to the subjective view - a kind of neutral monism. It almost seems that carelessness and inattention alone afford a remedy - the remedy of course of allowing ourselves to have any idea at all of what could fill in space (Blackburn, 1990, p. 65).

In line with Molnar's thinking, what we are missing, according to Blackburn, is that events which we conceive of as categorical do not really play any role in scientific understanding. Nor do they ground anything. As Blackburn notes, our assuming categoricity is a means of escaping the concept of a world with 'only powers without end', and the discomfort that this causes (1990, p. 65). However, it is worth askingparticularly given how 'weird' the universe looks through the lens of Quantum Mechanics - whether modern physics has removed the option of deciding between theories on the basis of an intuitiveness-comfort rating. As argued in Chapters 1, 12 and 14, there are good reasons for denying the existence of fundamental particles, particle-hood and categoricity.

Denying that either objects or categorical properties exist at the fundamental level requires re-defining power as the ability to affect or be affected. Because such power does not rely upon a mediating bearer or being grounded in the categorical, I refer to it as 'bare power'. Why reject power-bearers in favour of 'bare' power? Allowing for one brute type is ceteris paribus better than positing two brute types, such as power plus categoricity, which would have to be somehow 'tied' together. Moreover, explaining the tie between power-bearers (or ground) and power itself has so far proven very difficult, as evidenced by the ongoing debate in the theory of properties and discussed in Section 3 of this Thesis (Armstrong, 2004a; Baxter, 2001; Bradley, 1897). In examining the Identity Theory of Properties, I have also contended that identifying powers and non-powers in terms of singularised properties (called 'power-qualities') is unsuccessful in justifying how the two seemingly different 'natures' (see, Sparber, 2006) can be fused to represent one and the same property. As noted in Chapter 7, one difficulty with proposing two types of property is to defend 
the claim that non-powerful properties play a causal role of some sort, since roleplaying appears to be enacted by an agent of power.

Avoiding the need to explain how power and non-power sit together at some fundamental level appears to be an advantage of monist positions. Categorical Monism, which defines the world in purely categorical terms, has endured much debate. ${ }^{40}$ The main point of contention is whether one can satisfactorily explain the world without irreducible power featuring in it. Armstrong admits to Categoricalism being a 'soft' theory of power in light of its reliance on the laws of nature to supply the nomic necessity required for strong causation (Armstrong, 2004b, p. 142). As discussed in Chapter 3, Martin, Herbert Hochberg and Alexander Bird each offer reasons why Categoricalism fails to provide an adequate account of such necessity, given that the laws described in Categoricalism are contingent ones.

Power-maximalism, the monism as represented by Sydney Shoemaker's Early Causal Theory of Properties, faces the regress arguments discussed throughout this Thesis. Shoemaker's later response (1998) introduces quiddity into the notion of properties in order to circumvent a reduction of powers to properties, and vice versa. Thus, his position can no longer be described as Power-maximilist.

In Chapter 5, I noted that rather than revising the Causal Theory of Properties by introducing quiddity, a viable alternative would have been to posit FoundationMonism, which entertains only a single, powerful entity as fundamental. Harré's Great Field (1970; 1975), described in Chapter 12 of this Thesis, represents such a view. The Great Field is comprised of Parmenidean Individuals, which are not property bearers, but instead represent pure power. Power-bearers are rejected in favour of 'bare' power. There is no issue of what individuates these entities, since they are not strictly individuated. While we can talk of them as individuals for pragmatic purposes, their pseudo individuality is in virtue of being singularities of potential within the one entity of the Field.

\footnotetext{
${ }^{40}$ Discussions on Categoricalism include Armstrong, 1997b, 2001; Armstrong, 2004b; Armstrong, 2005b, 2005c, 2006; Armstrong et al., 1993; Baxter, 2001; Bird, 2005b; Crane et al., 1996; Dodd, 1999; Ellis, 2001a, 2002; Hochberg, 1999b; Lewis, 1986; Lewis, 1992; Lewis, 1999; Molnar, 1999; Molnar et al., 2003; Mumford, 1998, 2005; Prior et al., 1982; Rissler, 2006; Sider, 2005; Simons, 2005.
} 
A theory positing bare power as the sole fundamental, escapes the difficulties posed by fundamental categorical properties-described in Sections 1 to 3 of this Thesis-in accommodating strong causality. A successful defense of this view, however, requires at least: first, giving a suitable account of 'substance' (or whatever plays the substance role); and second, explaining the ostensible primacy of spatial properties with respect to the perceived individuation of distinct objects, without recourse to the qualitative. The rest of this Chapter details the former account, while Chapter 14 turns to the latter.

\subsection{Substance}

Some, for example Bertrand Russell, view General Relativity as precipitating abandonment of the traditional concept of matter. Outlining the theories of atomism (Newton) versus the aether (Descartes), Russell notes that the concept of matter, although associated with 'substance' in metaphysics, is not required for the examination of phenomena (Russell, 1925, p. 208). Asserting that substance is an outmoded concept, he reacts to Substance-attribute Theory whereby distinct objects persist through changes in their properties. He writes:

I draw the conclusion that science is concerned with groups of "events," rather than with "things" that have changing "states." This is also the natural conclusion to draw from the substitution of space-time for space and time. The old notion of substance had a certain appropriateness so long as we could believe in one cosmic time and once cosmic space; but it does not fit in so easily when we adopt the four-dimensional space-time framework (Russell, 1959 [1927], p. 286).

For Russell, the world is constructed out of 'events' (1959 [1927]) defined as 'entities or structures occupying a region of space-time which is small in all four dimensions' (1959 [1927], p. 286). Construing them literally in terms of topology, we can read them as more primitive than structured entities such as electrons or protons (1959 [1927], p. 9); and in a sense they are more primitive than spacetime itself (1959 [1927], p. 299). Russell views his events as 'continuous with percepts' in a way that 
implicates percepts as primary. Since it is not within the scope of this Thesis to discuss the Russell's notion of primitive percepts, I parenthesise this step and begin by simply illustrating his concept of an event in topological terms. (Russell's description of events and their contribution to spacetime points is based on the mathematics he names 'analysis situs' (1959 [1927], pp. 290-308), otherwise known as 'topology' or 'functional analysis'.)

In a 3-dimensional Euclidean space, consider the array of all spheres of some given radius, and let any group of them be defined as 'co-punctual' if any four from the group enclose some common region. Furthermore, let any co-punctual group be defined as 'punctual' if it cannot be numerically enlarged. Then the points of the space correspond one-to-one with punctual groups of spheres, and this holds if the spheres are distorted in any lawfully continuous way. Analogously, the pointmoments of Minkowski spacetime are constructible via co-punctual groups of five events, giving rise to the possible metrics of General Relativity. Russell's primitive events exist prior to spacetime, representing an ontology whereby the spacetime manifold emerges from entities rather than the other way around. Russell proceeds to extract a geometry compatible with 'causal lines' in modeling the physical world (Russell, 1959 [1927], p. 186). This is an early example of an ontology in which spacetime is emergent rather than fundamental.

Importantly, for Russell, the spacetime point is not a substance, and his view concerning traditional notions of substance in scientific explanation parallels Blackburn's view rejecting the inclusion of the categorical for such purposes. Russell writes:

The question is: Are electrons and protons part of the ultimate stuff of the world, or are they groups of events, or causal laws of events? We have already seen that the physical object, as inferred from perception, is a group of events arranged about a centre. There may be a substance in the centre, but there can be no reason to think so, since the group of events will produce exactly the same percepts; therefore the substance at the centre, if there is one, is irrelevant to science, and belongs to the realm of mere abstract possibility (1959 [1927], pp. 244-245). 
The idea of matter as permanent units persisting through time is replaced, in Russell, by that of the persistence of causal laws. To explain, without the concept of substance, how we perceive objects as extending spatially and/or temporally, Russell notes our recognition of causal chains preserving contiguous groupings of events (1959 [1927], p. 284). These casual chains provide the continuity and intrinsic unity important to the notion of persistence. Intrinsic unity is considered by him to replace material identity. He writes, '[t]hus the persistence of substance is replaced by the persistence of causal laws, which was, in fact, the criterion by which the supposed material identity was recognized' (1959 [1927], p. 285). Developments in theoretical physics, claims Russell, undermine the metaphysical status of persistent units of matter (Russell, 1985, pp. 12-13).

Russell considers material objects in terms of groupings of his events (1959 [1927], p. 320) arranged about 'crinkles' in spacetime whose influences diminish with greater distance; and which are superimposed to give the metrical structure of any region (1959 [1927], pp. 326-327). Like Russell, Harré questions the notion of primitive spacetime. As discussed in Chapter 12, he posits the idea of a field being ontologically, if not conceptually, prior to spacetime (1973, p. 223). I suggested that, for Harré's Great Field, variation in 'Influence' or potential allows for an intensive, rather than extensive, spatiotemporal distribution of topological features. The contours associated with a singularity provide a scale upon which 'distance' can be imposed as a continuous, well-ordered function of potential magnitude rather than as spatial extension simpliciter.

A challenge for the pure-power theorist, such as Harré, is to explain substance without recourse to fundamental categoricity. As Russell points out, our notion of matter is traditionally tied very closely to the idea of substance $(1925$, p. $208 ; 1959$ [1927], p. 286), but if qualitative substance is denied, then an account is required of what plays the 'substance role' in this manifestly qualitative world. Paul Teller (1995) notes three such roles: i) it allows an object to have an identity independently of its properties; thus ii) it explains the persistence of an object through changes in its properties; and iii) it provides a ground for properties. A Foundation-monist view, according to which there is only a single entity comprised of pure-power, must 
necessarily be a bundle theory of properties. In terms of rejecting some bearer, Harré's ontology does not allow any fundamental other than pure power. His Parmenidean Individuals comprise the fundamental. Moreover, no fundamental categorical properties can be said to ground pure power entities. The three roles above, therefore, do not apply, since they build-in a Substance-Attribute view to the discussion of how substance may be considered. Howard Robinson, however, outlines eight marks of substance that may be useful in appreciating how a pure power theory might engage with what has been previously seen as the role of substance (Robinson, 2004). Robinson's system allows his marks of substance to be drawn upon with more or less emphasis depending upon the theoretical stance taken. They describe substance as:

i) ontologically basic;

ii) relatively independent and durable (in some cases absolutely so);

iii) the subject of predication and the bearer of properties;

iv) the subject of change;

v) typified by things we normally classify as objects, or kinds of objects;

vi) typified by kinds of stuff;

vii) enduring particulars that give unity to our spatiotemporal framework, and the individuation and re-identification of which enables us to locate ourselves in that framework; and

viii) those entities, in a given system, that are crucial from the teleological or design perspective of that system. By the term 'crucial', Robinson means 'other things exist either to constitute them or to provide a context of operations for them'.

Robinson compares various major ontological stances concerning substance against these criteria. Early atomists, for example, emphasised criteria (i) and (ii) and, it can be argued, (iii) and (v); Plato's Forms comply with (i), (ii), (v) and (vi), but do not conform very well to (iii) and (iv); Aristotle's views as canvassed in Categories (Aristotle et al., 1938) can be thought to favour (v) over (vi), and so forth. If we combine the Categories with Metaphysics (Aristotle, 1960), Robinson concludes that Aristotle satisfies (i) through to (vi), although barely meeting (vi). 
Comparing Harré's Parmenidean Individuals-representing FoundationMonism - to Robinson's criteria for substance, we can see that they are ontologically basic, thus satisfying mark (i). Since they are immutable, mark (ii)—independent and durable — is particularly emphasised. Mark (iii) — the subject of predicates and bearers of properties - is partially fulfilled since the Parmenidean Individuals are capable of being the subjects of predication. They are predicates, for example, in the statement, 'Parmenidean Individuals exert an influence'. However, they are not bearers of properties; rather, they are 'properties' of the Great Field that they comprise. Parmenidean Individuals explicitly abandon (iv), the requirement that they be subject to change, since, as Harré emphasises, basic entities are immutable. However, the field that they comprise can endure rearrangement with respect to its distribution of potential densities. Marks (v) and (vi) also only partially apply, since Parmenidean Individuals belong to a 'kind' although do not constitute distinct objects in the 'normal' sense of 'object'. It can be argued that all marks assuming individual substances in terms of object-hood do not apply to a Foundation-monistic fieldtheoretic view. Marks (vii) and (viii), are emphasised heavily, although as discussed earlier, individuality of Parmenidean Individuals can only be individualised in a verbal sense for instrumental reasons. Since all but those marks that do not apply generally to Bundle Theories are satisfied (in varying degrees) by Harré's ontology, his Great Field can be said to play a substance-role, although fundamental categoricity is denied. Likewise, the field theoretic ontology that I defend in Chapter 14 holds up against Robinson's marks in a sufficiently robust way to argue that it allows an account of the substance role. I formulate this comparison in Chapter 14.

A contemporary debate with respect to substance concerns how to draw a distinction between substance and properties. Such a distinction may be in terms of dependence or else the ability to exist as a sole entity. A second issue concerns the individuation of objects. I discuss these issues in turn.

If we say that a distinction between substances and properties can be drawn in terms of dependence, we might formulate this as substance being somehow 'independent' of properties, but properties requiring substance for their existence. While this formulation is clearly not viable in general — since it is difficult to say how 
substances could exist un-propertied-Robinson suggests recasting the differentiation from the general case to instances:

Substances and events are distinguished from properties by the fact that properties are the kinds of things the instances of which depend for their existence on the particular substance or event by which they are instantiated, whereas substances and events are such as not to depend for their existence on particular instances of properties (2004).

Likewise, thinking of individual substances as particulars, David Armstrong differentiates particulars from properties in terms of instantiation: properties are the way that particulars are; particulars can instantiate properties, but properties cannot instantiate particulars. As Armstrong notes, however, there is a difficulty in describing instantiation without falling into Bradley's regress (Armstrong, 1997, p. 114). This was discussed in some detail in section 3.5 of this Thesis, and is sufficiently severe to encourage some to deny substance-attribute theories that purport properties to be instantiated by particulars. A possible alternative position is Bundle Theory, whereby bundles of properties comprise, rather than are instantiated by, objects. For Bundle Theorists, what distinguishes one bundle of properties from another might be an individuating property possessed by each bundle. Robinson notes that even if one holds the concept of substance as important merely for the instrumental purpose of discussing or analysing the manifest objects of the world, this does not negate the fact that substances might be nothing but collections of properties (2004). There seems to be nothing untoward about bundles undergoing rearrangement of their compositional elements, with their properties sorted somehow into accidental and essential ones.

A different kind of distinction between particulars and properties has been noted by Robinson, namely, Hoffman and Rosenkranz's (1994, 1997) differentiation in terms of the ability for a substance to be a sole entity (provided it is not mereologically comprised), an ability not shared by properties. Heil speculates about such a world, comprised of a single field whose Spinozian-like objects are modes or properties (Heil, 2003a, p. 189). The claim by Hoffman and Rosenkranz is that these worlds might suffice with a single substance, but that no world can do without 
multiple properties. This numerical differentiation distinguishes particulars from properties. As noted by Robinson, Penelope Mackie (2000) objects to this distinction on the grounds that it is not a sufficient condition for differentiating properties and particulars. The distinction does not hold, for example, for those possible worlds where there are multiple or complex objects. But is the distinction necessary for distinguishing properties and particulars? A Bundle Theorist working within a fieldtheoretic framework might object to the Hoffman-Rosenkranz distinction by claiming that it may be possible to posit a single fundamental property that grounds all others. Robinson offers the idea of a 'master property' of which all other properties are features. He suggests, for example, that for Descartes, the master property of matter is extension; whereas Newtonian model might adopt solidity (2004). The master property of a Foundational-monist world, such as Harré's Great Field, could be 'density' or 'distribution of intensity'. Since the Field is comprised of pure power, this would pertain to an intensive rather than extensive distribution, as discussed in section 12.2.5 of this Thesis. Taking a bundle view of such a world, we could then say, contra Hoffman and Rosenkranz, that such density is its sole fundamental property. Further objections might follow, that Hoffman and Rosenkranz could admit only a sole fundamental property, although insist on multiple properties nonetheless. In response, I would suggest that this situation would assume these multiple properties to be higher-order properties, yet built-in to the universe in some ontologically-robust way. I would suggest denying this assumption, however, in favour of imposing the 'Ontological Free Lunch Principle': higher-order properties are supervenient upon more basic properties, but do not represent additional 'furniture of the world'. Harré's Field manifests power in the form of attractive and repulsive forces, which are immediately re-interpretable, in the context of spacetime, as 'curvature'. This just is effectively the range of ways in which the Field can vary. The absoluteness of the speed of light entails that the temporal dimension is involved, and hence the curvature provides geodesics that correspond to the action of forces. Readily re-interpreted, again, as the 'structure' of the Field, we can see that it is intrinsically dynamic and powerful. However, such an account would only successfully act as a counterexample to Hoffman and Rosenkranz by the further limitation that structure is fundamental and that other 'spatiotemporal structures' - pointed out by Rickles and French (Rickles \& 
French, 2006, p. 1), to include causal structures, distance and so on-are higher-order features of the field. This idea is discussed in more detail in Chapter 14 of this Thesis. The claim that structure ought to be considered powerful is discussed in Chapter 14.4. Robinson's purpose for the master property is to act in the substance role as a grounding substratum. Taking 'solidity' as an example, he writes:

One possible resort is to treat solidity as the core or master quality and all the others as features of it. One would never ask what binds together a patch of colour and its shape, because the shape is the shape of the colour patch, and, though the shape of something can change, its shape cannot come away from it, like a separable component. Perhaps the shape, size and density of an atom are similarly features of the solidity. The quality solidity would then become equivalent to the notion of material stuff or material substance (2004).

As noted in 13.1, a pure Bundle Theory seems to lack the ability to account for how multiple properties might be held together to form an object. As Robinson notes, a regress seems to occur without something at base holding properties together into their bundles (2004). Thus, part of the substance role, plausibly played by the master property, appears to be an explanation for the bundling of properties.

Robinson also points to the individuation of objects as a second general role that substance plays. Such a role, however, does not apply to a Foundation-monist world, such as Harré's Great Field, that contains only a single object. If properties are to be considered tropes rather than universals, the role of substance is also relevant to explaining how these tropes may be individuated. However, a world that contains only a single fundamental property, i.e. structure, is in no need of such explanation.

\subsection{Summary}

This Chapter argues against the requirement that power be either borne by particulars or grounded in categorical properties. Instead, it defends a worldview according to which the fundamental entity is comprised of pure power, and effect can be considered in terms of an evolution of pure agency, understood in Harré's terminology as 'Influence'. There is an emphasis on differentiating between a purely 
dispositional world - dispositionality understood in terms of ability, possibility, capacity or potentiality - and one that is fundamentally powerful. It is argued that the categorical-dispositional distinction, like spacetime and the notion of possibility, arises only at higher-order levels, once persisting fermionic entities arise. How such entities may emerge from a base of pure power is the focus of Chapter 14 .

Throughout this Chapter, I draw a comparison between Rom Harré's Great Field - which I claim is shown to play a substance-role — and my own field-theoretic ontology. As I have claimed for Harré, my view also amounts to a Bundle Theory whose 'master' property is (intensive) structure. Other features of this fundamental represent higher-order properties. The world that I describe is purely powerful at fundamental levels, power being ontologically-robust and thus 'actual', but clearly not categorical, if by categorical we mean independent of causal power. The claim that structure should be considered powerful is also discussed in Chapter 14. 


\section{CHAPTER 14}

\section{DERIVING THE MANIFESTLY QUALITATIVE WoRLD FROM}

\section{A Pure-Power BASE: Light-LIKe NeTWORKS}

The constraint that Harré adopts for his Parmenidean individuals is that these fundamental entities are pure-power (Harré, 1970; Harré \& Madden, 1975). As basic 'entities', they involve neither haecceity nor quiddity, and obtain ontologically-robust status without grounding in anything categorical. The previous Chapter detailed how such a pure-power theory could plausibly account for a substance role without recourse to fundamental categorical properties. However, another requirement of a pure-power theory is to explain the ostensible spatial primacy of our perceived world in relation to the individuation of apparently distinct objects. In this Chapter, I flesh out how the manifestly qualitative world might conceivably emerge from a purepower base.

This Thesis contends that the categorical-dispositional property distinction arises only macroscopically, i.e. as a higher-order distinction. I also defend the claim that power requires no bearer to mediate its action; that spacetime curvature, corresponding to the presence of energy, evidences the exchangeability of effect in pure-power systems. The theory introduced in this section argues that what have traditionally appeared to be fundamental power bearers (e.g. fermions and objects derived from these building blocks) could actually be regions of a field ${ }^{41}$ comprised of complex networks of field fluctuations, circulating within extra dimensions. This would imply that ostensible power bearers are nothing over and above the respective networks, constituting pure power. Thus, the ostensible 'bearers' of power would be just perceptual products of power.

Because this account describes a scenario in which the fundamental entities are light-like processes that form networks, for purposes of efficiency I refer to what

\footnotetext{
${ }^{41}$ It should be noted that the term 'field' is used singularly throughout much of this Chapter, although it may refer to overlapping fields, e.g. the electromagnetic and gravitational fields. Subject to scientific investigation deriving a grand unified theory (GUT) encompassing all forces, my own account envisions, overall, a single field akin to Harré's unified and universal 'Great Field' - described in his 1973, p. 227.
} 
follows as the Light-like Network Account, LNA for short. This is not intended as a scientific theory or model of the physical world, since a great deal of work would be required before it could be recognised as such. Rather, it merely constitutes a plausible approach to metaphysically accounting for the manifest world while starting from a pure-power base.

\subsection{Primitives}

I begin with the idea that field fluctuations (i.e. force carriers or gauge bosons) - lightlike processes with a spacetime interval of zero and no rest mass - are suitable candidates for fundamental 'entities'. ${ }^{42}$ I also refer to them as 'neo-Parmenidean individuals' in deference to Rom Harré. As described in Chapter 12 of this Thesis, Harré and Edward H. Madden propose a field-theoretic view in which the underlying 'Parmenidean' primitives are unchanging, immutable, pure-power entities that give rise to higher-order, qualitatively-describable and changeable 'Aristotelian' individuals, which then constitute complex, manifest objects (Harré, 1970; Harré, 2001; Harré \& Madden, 1975; Madden \& Hare, 1971; Madden \& Sachs, 1972). A key difference between Harré's Parmenidean Individuals and the neo-Parmenidean Individuals of LNA concerns spatiotemporal extension. While Parmenidean Individuals are not extended in space, they have the time-like extension of persisting singularities. However, Harré's treatment is not even-handed with respect to space and time. If Parmenidean Individuals - as pure power — should have no extension in space, then why should they be assigned extension in time? In this respect, LNA's basic entities, which correspond to the gauge bosons of theoretical physics, part company with their Parmenidean cousins.

The Standard Model of particle physics describes four types of gauge bosons; the elementary carriers of the four fundamental forces. ${ }^{43}$ Photons carry the electromagnetic force. $\mathrm{W}$ and $\mathrm{Z}$ bosons carry the weak interaction involved in quark

\footnotetext{
${ }^{42}$ In referring to 'individuals', 'entities' and 'particles', I use the terms loosely, in line with my earlier objection to particle-hood. In the sense that fluctuations are of the field - the sole underlying entitythey are not distinct from it. They also can be seen as manifestations of power that affect the field, and hence one another. Just as Harré refers to his Parmenidean Individuals 'verbally' as entities (outlined in Chapter 12), I follow suit.

${ }^{43}$ Actually, there are fewer than four, since the weak and electromagnetic forces have been shown to be different forms of the same, and it is posited that all the forces will eventually be resolved into one.
} 
flavour changing. Gluons carry the strong interaction involved in the cohesion between quarks, antiquarks and gluons, within protons and neutrons. Gravitonswhose existence is supported by Quantum Mechanics, although not directly confirmed - carry gravity. Since the worldlines of force carriers correspond to a spacetime interval of zero, they are neither continuously space filling nor persistent. ${ }^{44}$ That is to say, in no coordinate frame of reference do they possess either purely spatial extension or purely temporal extension.

Gauge bosons travel at the speed of light - they are 'light-like' entitieswithout rest mass. (As a 'massy' object accelerates, its mass/energy increases such that this would be infinite at the speed of light. Hence, only things without rest mass can travel at the speed of light. ${ }^{45}$ ) Light-like processes, or events with a light-like separation, have a spacetime interval of zero by the formula: $\mathrm{Q}^{2}=c^{2} \Delta t^{2}-\Delta s^{2}$, where Q represents the spacetime interval, $c$ is the speed of light squared, and $\Delta t$ and $\Delta s$ represent changes in time and space coordinates, respectively. Providing the speed of light is defined as $c=1$, two events are separated by zero spacetime interval when the space between them exactly matches the time between them. Always at light speed, a gauge boson, at any moment, occupies zero space; and at any point, occupies zero time. Thus it has no spatiotemporal extension. In a Minkowski diagram, light-like processes - in contrast to time-like or space-like processes-are represented by 45 degree worldlines. Events connected by time-like processes, at less than 45 degrees from the vertical axis, are causally related. A vertical worldline represents an object at rest in space relative to the frame of reference, yet persisting through time. Events with space-like separation, or $\mathrm{Q}^{2}$ less than zero, can have no causal relationship. ${ }^{46} \mathrm{~A}$

\footnotetext{
${ }^{44}$ There is no absolute fact as to how much spacetime is involved between the occurrences of emission and absorption events pertaining to the transmission of force, since this varies with the frame of reference. The extension of such worldlines can be invariantly characterised, however, in terms of 'action', whose units are those of Planck's constant.

${ }^{45} \mathrm{~W}$ and $\mathrm{Z}$ bosons are thought to have rest mass of a kind, however the Higgs field has been postulated to account for this.

${ }^{46}$ Space-like intervals can, however, accommodate pseudo-processes that appear to move faster than the speed of light, e.g. an illuminated spot from a distant torch, moving across a brick wall. Bertrand Russell outlines two criteria for the existence of space-like processes: First, they are not causal; and second, there is a common ancestor responsible for each component of such a process (Russell, 1954 [1927], pp. 313-314). Given this description, we can characterise a pseudo-process as a coordinated change of state amongst mass/energy contiguities which occurs faster than the speed of light. However, this is not constitutive of the mass/energy contiguities themselves, and does not engage causally in the
} 
horizontal worldline might be construed to represent a state of rest in time, with extension through space, but this corresponds to nothing physical.

In addition to gauge bosons, we may regard spacetime's dimensional topology (incorporating length, breath, height, time and how they interconnect) as primitive. In our everyday experience, each spatial dimension represents an arbitrarily orthogonal direction of displacement, but the existence of more dimensions would simply mean the availability of further orthogonalities. In our natural experience of the world, the dimensional topology of spacetime consists of three dimensions of space and a single, directed dimension of time. The idea of a fifth dimension was first put forward by Gunnar Nordström in 1914, in an attempt to derive gravity from electromagnetism (Ravndal, 2003). In 1919 Joseph Larmor, to counter the Theory of General Relativity and to account for gravitation in terms of an extra dimension, also proposed a fivedimensional theory (1919, p. 354). Daniela Wuensch (2003) says that because both Nordström's and Larmor's theories used an extra Minkowskian-type or scalar spatial dimension, their attempts failed to explain the deflection of light in a gravitational field, and consequently their theories did not attract much attention (2003, p. 526).

Following these attempts, a five-dimensional theory was also put forward by Theodore Kaluza, ${ }^{47}$ submitted in 1919 but published in 1921 with the endorsement of Albert Einstein after a two-year waiting period (Kaluza, 1921). Kaluza's theory differed from those of Nordström and Larmor in that it was based on the Riemannian metric. Whereas a Minkowskian metric is scalar, the Riemannian metric employs a vector space that allows for curvature in describing positively curved spacetime; and Kaluza's use of it overcame the limitations of its forebears. Kaluza's introduction of the fifth dimension was conservative in that it was introduced for instrumental purposes such as mathematical expediency. Nonetheless, his main aim was to unify the electromagnetic and gravitational forces, and he was successful in accounting for charge in terms of a compacted, tightly 'curled-up', dimension, if charge is regarded as corresponding to motion within such a dimension. Wuensch notes:

world. Rather, it can be said to supervene on the mass/energy contiguities together with their antecedent patterning; and thus is not itself ontologically robust.

${ }^{47}$ See also: Gribbin, 2007; Randall, 2005; Wuensch, 2003. Translated in English, the title of Kaluza's Thesis is 'On the Unity Problem in Physics'. 
Kaluza proved that the five-dimensional gravitational equations, in fourdimensional space-time split into the Einstein and Maxwell equations... What appears to us as two separate interactions, gravitation and electromagnetism, is actually just one gravitation-like interaction in five-dimensional space...because we live in a four-dimensional space-time we only experience the fifth dimension as two separate interacting forces. What appears to us as electromagnetic interactions is in fact a projection from the five-dimensional gravitation (Kaluza, 1921, p. 971; Wuensch, 2003, p. 527).

Oskar Klein (1926) extended Kaluza's model in application to the physical world (Krauss, 2005) and assumed a quantum rather than classical field (van Dongen, 2002). In the ensuing Kaluza-Klein model, an extra, finite dimension of space is curled up at every spacetime location. It is 'periodic' such that motion in the curled up direction returns to the starting place. Outstanding issues concerning the impact of the finite or 'frozen' radius of the compacted dimensions with respect to the dynamic requirements of General Relativity ${ }^{48}$ (Biswas, 2003; Smolin, 2006, pp. 38-53; van Dongen, 2002), together with the overwhelming 1930s focus on Quantum Mechanics, resulted in unobservable dimensions being largely left aside until String Theory reinvigorated the concept. (For further discussion on Kaluza-Klein dimensions also see, Aitchison, 1991; van Dongen, 2002; Weingard, 1991). Higher-dimensional frameworks are presently being re-examined and 'Kaluza-Klein Theory' now generally refers to the geometrical representation of fields in more than four dimensions (Gribbin, 2007, p. 162).

LNA employs the Kaluza-Klein theory to suggest how gauge bosons might be engaged in circulation networks that give rise to fermions and the manifest substantial world. Without speculating on how many, LNA suggests that some extra, curled up spatial dimensions reside at every 'spacetime point' (Kaluza, 1921, p. 971; Wuensch,

\footnotetext{
${ }^{48}$ Lee Smolin argues that Kaluza-Klein dimensions need fixed radii to achieve the unification of gravity and electromagnetism, and are thus incompatible with General Relativity (Smolin, 2006, pp. 47-48). However there is good reason to suspect that fixed radii only apply to the application of compacted dimensions with respect to continuous fields. Jeroen Van Dongen shows that when quantised fields are assumed, the issue concerning fixed radii no longer applies. Much ongoing work is being conducted to explore the modification of Kaluza-Klein dimensions in this respect, including: Biswas, 2003; Bringmann \& Hofmann, 2007; Dzhunushaliev, 2008; Dzhunushaliev \& Myrzakulov, 2005; Kriz, 2005; Van Dongen, 2002.
} 
2003, p. 527). In extrapolating from the Kaluza-Klein attempt to account for electromagnetic charge in terms of one compacted dimension, LNA conjectures that a similar story applies to all conserved physical quantities. This portrays each form of gauge boson as circulating in Kaluza-Klein fashion; reflecting Gribbin's speculative picture of a photon as a 'ripple' in the fifth dimension, a $\mathrm{W}$ boson as a ripple in the $6^{\text {th }}$, a $\mathrm{Z}$ boson in the $7^{\text {th }}$ and so on, including combinations (Gribbin, 2007, pp. 105106). This makes sense of the emission and absorption of gauge bosons by fermions; say, photons by electrons. As Icke notes, 'the old problem: if an atom drops to a lower energy state and emits a photon, where was the photon before that? The answer...the photon was in another world, another "abstract space", and has become apparent at the juncture between the space(s) containing the single electron' (1995, p. 182). LNA sees this 'other space' as the compacted dimensions in which gauge bosons circulate. In this view, virtual gauge boson are neither created nor annihilated, since this would be inconsistent with postulating them as basic, 'neo-Parmenidean' entities. Rather, they exit and enter-in being emitted and absorbed - the respective circulation networks. The requisite change in trajectory involves the exchange of energy and quantum states, corresponding to the causal efficacy of gauge bosons as 'messengers' and force carriers (Davies \& Gribbin, 1992, pp. 230-231).

In the example of an electron, the networks themselves may be thought to comprise the gauge invariant conserved quantity of charge, which, under a local symmetry transformation (discussed shortly), gives rise to the electromagnetic field. A charged area is surrounded by a 'sea of virtual photons' or 'messenger bosons' which can be pictured as being emitted from and absorbed by the circulation networks. Importantly, this account unifies conserved quantities, their associated gauge bosons and the interplay between them. We arrive at a fermion (e.g. electron) without recourse to anything qualitative or categorical.

\subsection{Circulation Networks}

I now focus on how a manifestly qualitative world might arise from a pure-power base. The principle of symmetry figures prominently in modern physics. Certain 'features' of both gauge bosons and fermions represent conserved quantities which remain constant in a closed system. Examples include: charge (associated with the 
photon), isotopic spin (associated with $\mathrm{W}$ and $\mathrm{Z}$ bosons and gluons), and energymomentum (associated with gravitons). An example of global symmetry can be given by electromagnetic charge: if all positive and negative charges were reversed, no discernible difference in the universe would exist. We can say that a quantity is 'gauge invariant' if, in changing some aspect of the relevant system, the measure of that quantity does not vary.

Global symmetry applies to whole systems, but where only part of a system changes we get local symmetry transformations. Vincent Icke visually represents local symmetry transformations through the metaphor of a tablecloth being twisted beneath a fixed point while the edges are held in place (1995, pp. 176-185). The tablecloth develops creases (representative of force lines) that radiate out from the fixed point. As Icke notes, the disturbance of any local symmetry generates a compensatory force, and each respective force can be described in relation to some gauge boson (Icke, 1995, pp. 182-183). For the electromagnetic field, the associated gauge boson is the photon. Gribbin offers a discussion of the electric current associated with a magnetic field (2007, pp. 104-106). A local symmetry transformation occurs, but electric charge is a conserved quantity, and so a counteracting magnetic force is presented. Symmetry is restored (or better, conserved) through the electromagnetic field by forcesessentially changes 'imposed upon' respective field fluctuation trajectories in conservation of the physical quantities that they represent. A similar story can be postulated in terms of the other three primary forces - the strong, weak and gravitational. In the case of the strong force, the associated gauge boson is the gluon; the weak force is associated with $\mathrm{W}$ and $\mathrm{Z}$ bosons; and gravity with (hypothesised) gravitons.

At this point, I emphasise again that LNA does not pretend to model physical reality, but as an attempt to establish consistency among metaphysical considerations of reality, it echoes certain prominent contemporary scientific research, which lends credence to its premises. On this footing, LNA presents an alternative, broadly compatible interpretation of symmetry at the fundamental level. Put simply, it extrapolates that a change in a fermion occurs within the compacted spaces where field fluctuations circulate, entailing that a gauge boson either enters or exits the network. The idea that fermions are thus 'made of' gauge bosons, however, sets a 
challenge for LNA: the proposed micro-topology somehow must account for bosonic whole-integer spin (or single-loop phase path), as opposed to fermionic half-integer spin (or double-loop phase path). This calls for some topological development whereby extra dimensions are made available, beyond the $3+1$ dimensions of everyday spacetime. As Icke explains, this is needed because given 'the right to step out into another direction, we can twist and untwist the phase paths of bosons and fermions...to change angular momentum by half a unit' (Icke, 1995, p. 279). This very possibility is explored by Supersymmetry Theorists in modern physics, searching for a 'Theory of Everything'. We can see that if fermions could be explained in terms of the effectively permanent outcome of a topological field fluctuation, as accommodated by LNA, then it would be natural to regard them as gauge bosons in disguise. A note of supporting evidence comes from the fact that when a fermion meets its antimatter (chiral) counterpart, which is presumably of opposite 'twist', their mutual annihilation results nothing but gauge bosons.

Local symmetry transformations tell us that conserved quantities are tied inextricably to fundamental force-fields, which can be described in terms of the gauge bosons associated with these fields. An important question is raised: where do the conserved quantities come from? LNA offers a neat answer: circulation networks of gauge bosons form conserved quantities. If an electron-representative of a fermion - were described as a set of conserved quantities partly constituted by a field of 'virtual' photons, this would be without recourse to anything categorical. Just as electrons, interpreted as charged regions of virtual photons, might boil down to manifestations of the field, so the field would be ontologically prior to fermions. The denial of fundamental status to fermions is not unprecedented. As discussed in more detail shortly, James Dodd recounts a history of attempts to present models showing quarks and leptons to be themselves comprised of smaller components (Dodd, 1984, p. 172). ${ }^{49}$ Kaluza and Klein opened up the possibility of accommodating conserved quantities, like charge, spin and energy-momentum, in terms of extra compacted dimensions. LNA suggests understanding conserved quantities as networks of

\footnotetext{
${ }^{49}$ One problem that arises with pre-quark models concerns accounting for the great energy required to confine components, compared to the relatively little energy actually had by fermions. Programs designed to deal with such problems are, however, outside the scope of this paper, which may in any case avoid the issue.
} 
circulating gauge bosons giving rise to fermions and hence the manifestly substantial world.

\section{Figure 14.1: 'Barber Pole’ Compared With Gauge Boson Path ${ }^{50}$}

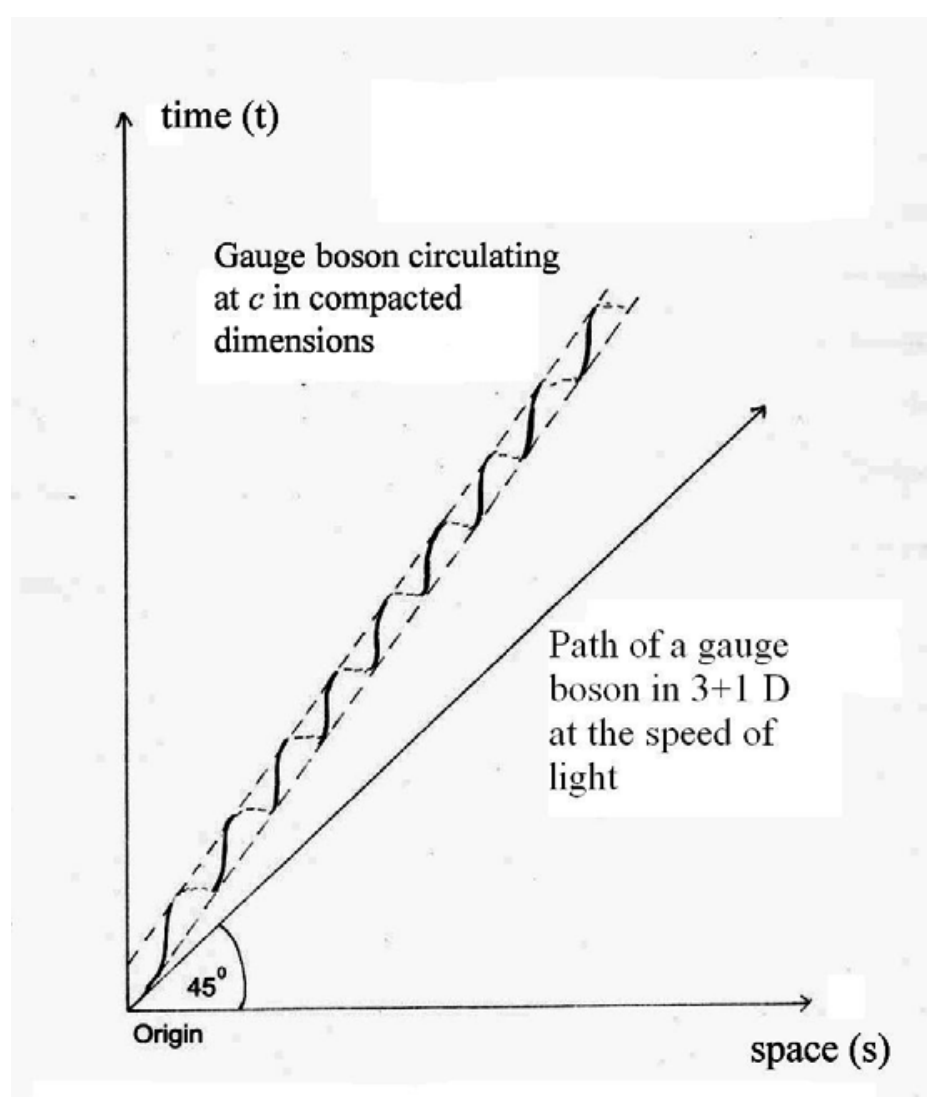

\footnotetext{
${ }^{50}$ Note: The barber pole leans over at angle $\arctan (\mathrm{v} / \mathrm{c})$. If its radius were fixed, then the $45^{\circ}$ spiral stripe (gauge boson path) would have a horizontal component greater than its vertical componentcontrary to physics. However, the components remain equal if the barber pole cross-section contracts in the direction of motion as per Special Relativity. If $\mathrm{x}$ and $\mathrm{y}$ are the two respective spatial dimensions and $t$ is that of time, then we have:

$\mathrm{v}^{\prime} \mathrm{x}=(\mathrm{v}-\mathrm{c} \sin (\mathrm{t})) /(1-(\mathrm{v} / \mathrm{c}) \sin (\mathrm{t}))$,

and $\mathrm{v}^{\prime} \mathrm{y}=\mathrm{c} \cos (\mathrm{t}) \sqrt{1}-(\mathrm{v} / \mathrm{c})^{2} /(1-(\mathrm{v} / \mathrm{c}) \sin (\mathrm{t}))$,

such that $\left(v^{\prime} x\right)^{2}+\left(v^{\prime} y\right)^{2}=c^{2}$ as required.

This bears on the wavelength and thus the energy-momentum of the circulating gauge bosons in accord with $\mathrm{E}=\mathrm{E}_{0} / \sqrt{ } 1-(\mathrm{v} / \mathrm{c})^{2}$ where $\mathrm{E}_{0}$ is the relevant absorption/emission energy in relation to a network at rest. (For further explanation of addition of velocities in Special Relativity, see Hartle, 2003, p. 71).
} 


\section{Figure 14.2: Network Trajectories of Gauge Bosons}

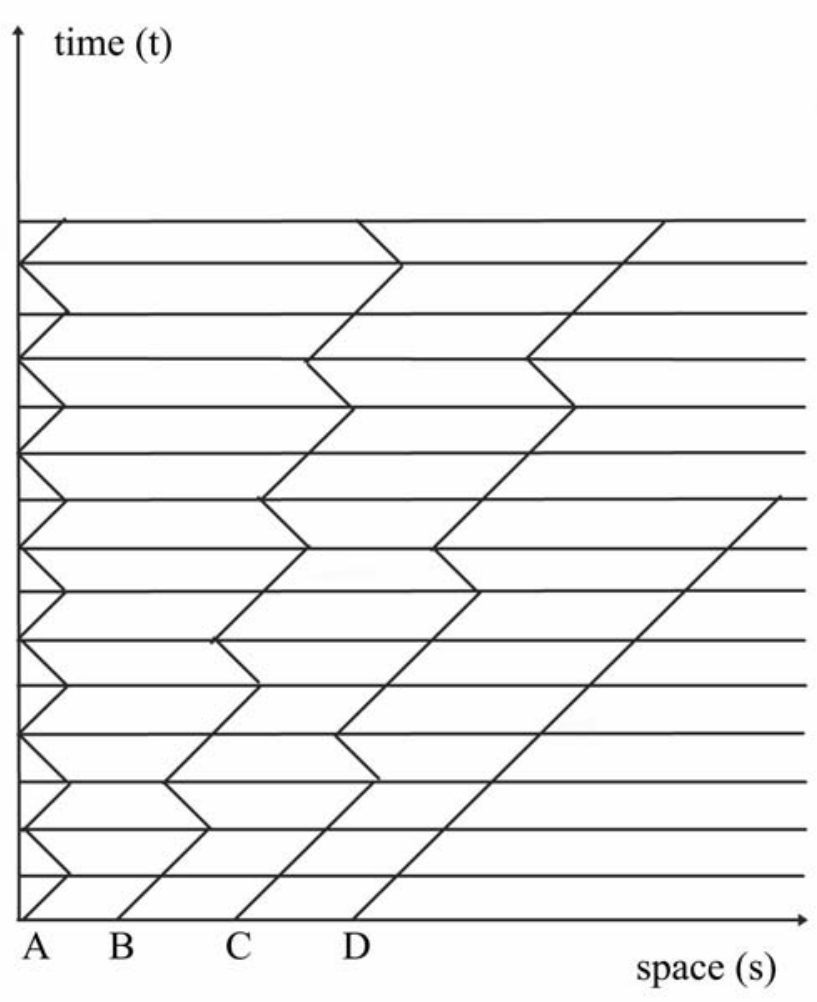

A 'barber pole effect' illustrates how such networks offer an explanation for massiness, even though the constituent gauge bosons lack rest mass. Figure 1 is a schematic Minkowskian representation of a gauge boson circulating within one compacted dimension. The effect can be likened to moving in a spiral up a barber pole, ending up at the starting place in terms of space, although at successively later times. A cross-section of the barber pole is just a circle (or ellipse), the simplest compact space orthogonal to everyday 3-space. In a three-plus-one-dimensional universe, the light-like process in Figure 1 applies. However, if compacted dimensions are involved, then while a gauge boson travels at the speed of lightmaintaining a spacetime interval of zero in a 'five-plus' dimensional spacetime- it 
may circulate as part of some network whose displacement entails velocity less than $c^{51}$

Figure 2 schematically shows how the velocity indicated by the lean of the barber pole varies depending upon how, relative to a frame of reference, gauge bosons travel through the compacted orthogonal spaces. Barber pole A represents the path of a gauge boson as a network that is persisting through time with zero velocity; $\mathrm{B}$ and $\mathrm{C}$ represent the same thing at successively greater velocities; and for D the overall path is light-like. The velocity of the network (the barber pole) itself differs from case to case, although the circulating gauge bosons comprising the networks always have light speed.

In summary, first, field fluctuations would otherwise travel along geodesic paths in 3-space, but the compacted, tangent spaces allow them to circulate through a multi-dimensional micro-topology whose local structure is influenced by the 'original' field fluctuation, forming specific circulation patterns. The resulting networks are gauge boson absorbers and emitters. Second, any given network (e.g. charged region in a sea of virtual photons; a.k.a. electron) as a whole moves slower than the speed of light; representing a concentration of energy-momentum with associated rest mass, identifiable by type. Thus, from the frame of reference of any network, other networks appear 'massy'-persisting as the fermionic constituents of

\footnotetext{
51 Barber pole detail: Consider a Minkowski diagram with two graphically horizontal, spatial dimensions. On this diagram, a vertical barber pole represents a stationary light-like network-a particle-like object with zero relative velocity. A barber pole that is slightly leaning over represents a light-like network with non-zero velocity. Choosing units such that the speed of light is unity, then by convention any velocity is denoted by the symbol $\beta$-a value between 0 and 1 . The angle at which a barber pole is leaning over, from the vertical, then equals $\arctan (\beta)$. The red stripe on the leaning barber pole represents the path of a light ray, circulating within a one-dimensional micro-topology (in a circular or elliptical path). The stripe must be painted on the barber pole at a constant $45^{\circ}$ to the vertical. This implies that, for one complete spiral circuit of the barber pole's circumference, the total horizontal (spatial) distance is more than the total vertical (temporal) distance traversed by the paint brush. This is contrary to the analogous physical possibility for a ray of light, whose horizontal and vertical path-lengths must remain constantly equal. However, suppose the circumference of the barber pole is contracted in accord with Special Relativity. In this case, when the lean of the barber pole corresponds to the speed of light, its circumference is zero, and so the stripe is simply a $45^{\circ}$ straight line. Equality is thereby maintained between the spatial and temporal components. Given some suitable space contraction, with an associated time dilation, as a function of $\beta$, the required unit ratio would be maintained for all $\beta$ values - all relative velocities. Is the required function of $\beta$ simply supplied by Special Relativity? The mathematics required to resolve this question exceeds this author's capacity, but it might be useful to explore the robustness of the barber pole metaphor in this regard.
} 
qualitative objects. ${ }^{52}$ In this light, fundamental levels may be devoid of rest mass, while at supervening levels the appearance of networks gives rise to the manifest world. Hence, LNA supports both the recognition by Rovelli, that matter and spacetime cannot be fruitfully distinguished (1997, pp. 191-195), and Bird's claim that spacetime should be considered powerful (2005a).

Since LNA treats dimensionality as fundamental, it is important to the argument that compacted dimensions are not viewed as categorical in the sense of 'bordering off', 'directing' or 'containing' power in the way that qualitative structure is often portrayed. Everyday dimensions, for instance, do not border off motion. I may walk indefinitely in an arbitrary direction or orthogonally in two others, in accord with local spacetime curvature. Motion among compacted dimensions would be likewise unbounded, albeit of finite size, just as 3-space may be finite yet unbounded on the cosmological scale. Certainly, any compacted dimensions possess size, just as the universe at cosmic scale might be 'closed' and finite, but in any case, the compacted circumference would emerge from the field's intrinsic potential gradient. Moreover, lacking any location (its orthogonalities being 'everywhere'), there would be no object-hood about it.

The overall idea is gauge boson motion emerges along with spacetime curvature, both in macroscopic 3-space and throughout the micro-topology. While it is initially useful to separate talk of field fluctuations from discussion of the dimensional topology, they can be regarded as not strictly distinct. Rather, the dimensional orthogonalities together with field fluctuations could be considered interdependent and dynamically overlapping as each force carrier induces curvature, modifying the range of orthogonalities 'available' to itself and others. ${ }^{53}$ That is, gauge bosons - as primitive effects - could conceivably change the geometry and even the topology in terms of independent orthogonalities in which they affect and are affected. Consider, for example, a convergence of photons that increases the energy density in some

\footnotetext{
${ }^{52}$ Whereas photons would circulate among the microtopological paths that we identify as electrons, gluons would do the same for quarks. Of course, while photons do not interact with each other, gluons do so. We can speculate that this is due to their being fluctuations of distinct combinations of compacted dimensions. Another relevant speculation is whether the eight gluon colour combinations somehow might correspond to the eight possible curvatures of 3-space.

${ }^{53}$ Issues surrounding the problem of infinities, fixed versus relational spacetime background and research into Quantum Gravity are ongoing, but outside the scope of this paper. (For further discussion, see, Smolin, 2000; 2006.)
} 
spacetime region. The consequently greater gravitational curvature changes how photons may be absorbed and emitted by that region. In this picture, spacetime structure is interdependent and to some degree interchangeable with gauge-boson activity. That is to say, the underlying field structure of intrinsic orthogonalities and the field fluctuations (gauge bosons) need not represent distinct metaphysical categories.

Since LNA views the field as comprised of power rather than bearing power, gauge bosons and the dimensional topology are not considered to be 'bearers of properties' as described by Substance-Attribute Theory. In keeping with Robinson's suggestion of a master property, LNA suggests that the field be identified with the master property of 'power density', interpretable as field structure, and that all other features exist as higher-order properties of this property. For gauge bosons, being absorbed or emitted by the compacted dimensional micro-topology is merely a matter of changing trajectory whereby the physical quantities corresponding to any field fluctuation are conserved. That is, they enter or exit circulation networks of 'sibling' gauge bosons, which may require quantisation for geometric reasons. Thus the idea of the 'quantum' is not tied to that of 'particle' in any traditional sense of the term.

\subsection{Particles and Fields}

If we suppose absorption and emission events to be changes of trajectory for gauge bosons (or field fluctuations) such that they exit or enter, not nothingness, but extradimensional 'circulation paths', then quantisation itself might potentially be explained in dynamical terms rather than by merely inferring particularisation. Questioning the strict individuation of particles at fundamental levels removes the requirement for fundamental categorical properties to supply the means of individuating particulars. This, together with the difficulties introduced by incorporating fundamental quiddity, leads naturally to supposing that, if fundamental structure were to be built-in, perhaps this should happen in terms of a pure-power ontology.

As noted, particles have traditionally involved 'primitive thisness' (Teller, 1995, p. 29) or haecceity-described by Armstrong as that which individuates particulars; a unique inner essence over and above any properties (1997, p. 109). Michael Redhead calls this 'transcendental individuality' (TI) - the idea of entities as 
'bearers' of properties, or in Redhead's words, 'individuation that transcends the properties of an entity' (1982, p. 59). Denying strict particle-hood removes the need for haecceity. Thus Redhead and Teller's idea, that the 'quantum' is not tied to 'particle-hood' in any traditional sense, features in LNA (Redhead, 1982; Teller, 1982). It can be argued that favouring categoricity seems tied to the intuitively attractive idea of fundamental particularity (i.e. haecceity or primitive thisness). Physics shows that gauge bosons, for example, while they appear wave-like in terms of motion, engage in 'emission' and 'absorption' events involving discrete 'quanta'. Such phenomena lead us to often think of them as particles, since 'particle-hood' is intuitively associated with distinct individuality or basic particularity.

The reality of particles in modern physics and more recently in metaphysics is highly debated, however, with many in favour of abandoning the traditional concept. Redhead argues that a traditional dualistic approach adopts two categories of entity: particles and forces between them. We may ask whether particles can be reduced to forces, and/or forces to particles. He shows that by quantising a field, we give it a particle aspect. In Quantum Field Theory (QFT), while particles are created and destroyed, they are, as Redhead notes, 'just quantized excitations of particular modes of the field' (1982, p. 70). He likens them to the bumps in an active skipping rope, whereby quantisation does not entail that the field constitutes a collection of traditional particles (p. 70). Redhead provides an extended argument for why the distinction between 'field' and 'particle' can be tied to neither the distinction between boson and fermion, nor that between massless and massive fields (pp. 72-76). Photons, for example, have no rest mass, but because they carry energy and momentum, observes Redhead, we are inclined to treat them as substantial (p. 79). However, he writes, "it is not at all clear which is the "matter" particle and which is the "force" particle' (p. 80).

Redhead's attempt to address the dilemma involves retaining the concept of particle while questioning the distinction between substance and force. He posits 'ephemerals', described in terms reminiscent of Lewis Carroll's 'Cheshire cat' as 'entities which can be distinguished one from another at any given instant of time, but unlike continuants cannot be reidentified as the same entity in virtue of TI at different times' (p. 88). Since a collection of indistinguishable particles may be described as an 
ephemeral in Redhead's view, this encompasses fields. He writes, 'like the Cheshire cat, although the substantial particularity has gone, there remains a particle "grin". The elementary "particles" are not particles but they are also not classical-type fields. They are quantum fields - ephemerals with a particle grin' (p. 88). They are not classical fields, in this view, because they retain particle-like aspects such as energy and charge that come in discrete amounts (Teller, 1982, p. 108). Thus, according to Redhead, the 'particle' and 'field' concepts are underdetermined in QFT.

Paul Teller adopts Redhead as a starting point, but takes his 'too soft treatment of ephemeral particles' further, to abandon any role for particularity. Teller claims that the notion of 'particle' in QFT is a relic of overlooking the fact that a full description, as per Feynman diagrams, ${ }^{54}$ must depict superposition of all processes mediating between input and output. Partial or selective use renders the appearance of such diagrams as operating in terms of the particle concept to the exclusion of the superimposable field concept (1982, p. 109).

The argument for abandoning particles involves rejecting haecceity, or in Teller's preferred terminology, primitive thisness. He describes a hypothetical scenario whereby distinct particles (say, an electron and a proton) are distinguished by fixed, individuating properties. Teller argues that although it is 'natural' to read these fixed properties in terms of primitive thisness, attempts to formalise such a reading ${ }^{55}$ lead to 'surplus structure', a term employed by Redhead (Redhead, 1975, p. 88) to formally describe elements that are absent in the 'real world' (Teller, 1995, p. 25). That is, recognition of fixed properties will entail system components (e.g. nonsymmetric vectors) that lack real-world counterparts (pp. 20-26). This failure of reduction from theory to the natural world is argued by Redhead to show that elementary particularity in the traditional qualitative sense does not exist. It represents what he calls 'one of the most profound revisions in our ultimate metaphysical weltanschauung, that has been engendered by our most fundamental physical theory, viz. quantum mechanics' (pp. 61-62).

\footnotetext{
${ }^{54}$ A Feynman diagram symbolically represents sub-atomic 'particle' interactions according to all possible 'pathways'.

${ }_{55}$ For example, by using Labeled Tensor Product Hilbert Space Formalism (LTPHSF).
} 
Likewise, Carlo Rovelli holds that entities traditionally considered particles, such as electrons, quarks and so on, should be considered in terms of fields (1997, p. 191). Rovelli views particle-hood as a long-standing inference formulated in spite of the fact that the particle-aspect of quantum 'entities' has never been detected and might be undetectable in principle (1997, p. 191). For Rovelli, particle-hood as traditionally conceived appears unsustainable:

Indeed, a physical particle cannot be an extended rigid object, because rigid bodies are not admitted in the theory (they transmit information faster than light), nor can it be a pointlike massive object, because such objects too are incompatible with the theory (they disappear in their own black hole). Thus, understanding the physical picture of reality offered by general relativity in terms of particles moving on a curved geometry is misleading (1997, pp. 191192).

As Rovelli explains, particles cannot be rigid bodies with pure spatial extension, involving instantaneous transmission of effects from one side of the object to the other, faster-than-light processes being ruled out by Special Relativity. Explicitly, the spacetime interval $\mathrm{Q}^{2}=c^{2} \Delta t^{2}-\Delta s^{2}$ must be zero or positive for all physical processes. Thus, extension through space must accommodate some associated extension through time, and continuous, purely spatial extension is untenable (putting aside discussion of tachyons or pseudo-processes). Neither can particles be point-like. Taking Newton's Law of Gravity, $\mathrm{F}=\mathrm{G} m_{1} m_{2} / r^{2}=m a$, where $a$ is acceleration; reformulated to accommodate only a single mass we get, $a=\mathrm{G} m / r^{2}$. By this formula, point-like particles would, due to their zero radius, form 'singularities', representing loci of infinite gravitational acceleration, that are causally cut off from the universe.

This is also in keeping with Mark H. Bickhard's discussion of quantum particles and quantum foam, emphasising the transient nature of these 'entities' that defy traditional concepts of rigidity or spatial extension:

The uncertainty principle in quantum field theory yields not just virtual particles, but a vastly different notion of the basic vacuum in which the 
phenomena of physics are conceived of as taking place. In particular, the vacuum becomes a sea of continuous creation and annihilation of field quanta in accordance with the uncertainty principle - a foam of such creations and annihilations. The dynamics of quantum fields can be crudely conceptualized as waves of excitation moving in this sea of background activity (Bickhard, accessed 2008).

John Gribbin argues that the 'folk notion' of fundamental particles is basically a means to understand the mathematical laws describing fields of force, spacetime curvature and quantum uncertainty:

Over the past fifty years, physics has revealed a wonderland of a subatomic world, populated by all kinds of strange objects. We call those objects particles, for want of a better name...Such truth as there is in any of this work lies in the mathematics; the particle concept is simply a crutch ordinary mortals can use to help them towards an understanding of the mathematical laws. And what those mathematical laws describe are fields of force, spacetime curved and recurved back upon itself in fantastic complexity, and a quantum uncertainty when you try to peer at it closely (1998, pp. 51-52).

For Gribbin, electrons can be considered as 'energetic bits of the field', confined to a certain region by the uncertainty principle such that they can move only half the distance of their wavelength before they are reabsorbed into the region of the field that denotes the electron. Thus, he describes an electron as, 'a charged region embedded in a sea of virtual photons' (1998, pp. 61, 64). The confinement occurs in accord with an application of Heisenberg's uncertainty principle involving energy and time. In short, an energy variation multiplied by an associated time variation must be less than or equal to Planck's constant—which determines the size of the discrete packets (or quanta) in which field fluctuations occur. As described by Lawrence M. Krauss, the shorter the time of measurement, the less accurate - and therefore the greater the uncertainty of energy (2005, p. 108). In the case of an electron, at very short time spans such that the uncertainty in amount of energy is larger than the 
electron's rest mass, there could be more than one particle present, described as virtual particles. Krauss notes that no violation of the laws of physics occurs if more than one particle, e.g. an electron-positron pair, exists in a given region for a sufficiently short time before the two counterparts annihilate each other. ${ }^{56}$ Krauss describes what had previously been thought 'empty space' as a brew of boiling, bubbling, 'particle-antiparticle pairs popping in and out of nothingness' (Krauss, 2005, pp. 108-109). We arrive at a picture of an electron as a locus of electromagnetic field in which virtual photons are encapsulated for short durations of time, when they act as 'messengers'. The message is in the form of a disturbance which 'exerts a mechanical effect both on the receiving particle (action) and on the transmitting particle (reaction)' (Davies \& Gribbin, 1992, pp. 230-231). ${ }^{57}$

Harré may have considered an electron to be Parmenidean, but in keeping with my aforementioned even-handed treatment of space and time, I prefer to view Harré's Aristotelian individuals (1975, p. 96) as corresponding to fermions, comprising quarks, electrons and neutrinos, which have rest mass. If, as suggested, fermions are ultimately manifestations of the field, then it seems that the field, at bottom, is ontologically prior to them. The denial of fundamental status to fermions, although initially surprising, is not unprecedented. As discussed above, Davies, Gribbin and others describe the electron as a charged region within a sea of virtual photons; basically a field. James Dodd (1984, p. 172) recounts a history of attempts to present models showing fermions to be themselves comprised of smaller components. While there is no experimental evidence, thus far, supporting the notion that fermions are composites, there is also no disconfirming evidence, and some good motivations for

\footnotetext{
${ }^{56}$ In quantum theory epistemological and ontological concerns are not always readily distinguishable, as they are claimed to be in the macro world. Rovelli provides a useful discussion on 'information', noting that a complete description of the world would be 'exhausted by the relevant information that systems have about each other' (Rovelli, 1997, p. 201). If we consider ourselves to be 'systems' then the idea of an 'observer-independent description' of the world dissolves. As suggested by Rovelli, 'The state of a system is always a state of that system with respect to a certain observer' (Crane, 1994; Rovelli, 1997, p. 202).

${ }^{57}$ This raises the question of what might be the difference between virtual photons and 'real photons' (those that travel long distances, for example, from the sun to our eyes). This issue is still under considerable discussion. A view that there is no real difference between the two would reflect their equal mathematical treatment according to which both are essentially excitations of the field; and detected only in terms of force. 'Real' photons would be those not yet drawn into an electromagnetic field and thus not yet absorbed (until, of course, observed via some perceptual apparatus). The same considerations can be made for the other types of gauge bosons.
} 
postulating that they are divisible. First, there are too many types of these entities to comfortably accept them as fundamental. Second, as Dodd notes, 'we would like to explain the apparent three-generation structure of the quarks and leptons which introduces more-massive repetitions of the same basic set of quantum numbers' (1984, p. 172). Third, the charges on quarks are exactly one-third divisors of the electric charge. Dodd describes two models of fermions as composite: first, the Abdus Salam and Jogesh Pati model (1974) according to which 'preons' form quarks and leptons. Second, the Rishon model put forward by Haim Harari (1979) proposing that any quark or lepton is a combination of two primal rishons. The three generations of quarks and leptons could be merely different 'dynamical excitations of the same system' (Dodd, 1984, p. 172).

I have argued for rejecting haecceity (or primitive thisness), and the associated notion of strict particle-hood. However, a question remains concerning quiddity. Robert Black suggests that as haecceitism is for particulars, quidditism is for properties (Black, 2000, p. 92). Associated with categorical properties, it corresponds to their having some 'nature' independent of their causal roles, in much the same way that haecceity implies particulars having some nature over and above their properties (for further discussion, see, Bird, 2006; Black, 2000). In this Chapter, the issue of quiddity translates to the question of whether properties have some non-causal aspect. As mentioned earlier, LNA portrays gauge bosons (or field fluctuations) as resembling Harré's Parmenidean individuals in representing pure power. Harré's picture rules out their being extended in space, since that would make them identifiable by qualities such as shape and size, and thus subject to change (1975, p. 163); but they clearly possess extension in time. In the sense that numerical individuality may represent paradigmatic haecceity, temporal extension arguably represents paradigmatic quiddity, as follows.

Categorical properties are not powers and therefore do not generate effect, so their bearers (or bundles) must be capable of 'non-change'. Moreover, at the fundamental level, purely spatial extension is ruled out by arguments such as Rovelli's, discussed earlier. At the fundamental level, then, the remaining medium of change (or non-change) is time-neither change nor non-change exists apart from time. Accordingly, at this level, any categorical property must have temporal 
extension in order to be capable of being had and/or lost by its bearer (thus allowing for changes in bearers to occur). (Indeed, from Harré's viewpoint, such extension cannot be a property because his fundamental entities are pure power - but as neither a power nor a property, this is problematic; a point that ties in with my demand (sections 12.2.5 and 14.1) for even-handedness with respect to neo-Parmenidean Individuals). In any case, the property of temporal extension is thus associated with categoricity. Such temporal extension goes hand in hand with possessing rest mass, and informs Martin's (1997) definition of qualitative properties as those which afford our qualia - and which thus result in our being able to see, hear, touch, taste and smell-whereby extension seems mandatory for the processes of the world. Indeed, the Swinburne regress emphasises this expectation of properties to be 'observable', temporally extended and correspondingly massy.

We can track the association of rest mass with temporal extension. As the first step, from Special Relativity, presenting the familiar $E=M c^{2}$ more precisely:

$$
E=M_{0} c^{2} / \sqrt{ } 1-(v / c)^{2}
$$

where $E$ is energy, $M_{0}$ is rest mass, $v$ is velocity and $c$ denotes the speed of light. It is clear that as the velocity of a massy entity approaches the speed of light, its energy approaches infinity. Since infinite energy is not viable, we may say that only entities without rest mass can travel at the speed of light. ${ }^{58}$ Fermions must travel at less than light-speed. The second step is to show that things moving at less than the speed of light, such as fermions, necessarily possess rest mass, and are temporally extended in the above sense. From the above formula:

$$
E \sqrt{ } 1-(v / c)^{2}=M_{0} c^{2}
$$

Thus, given velocity less than $c$, the term $\sqrt{ } 1-(v / c)^{2}$ will be positive, so the presence of energy implies non-zero rest mass. Such velocity delivers a non-zero spacetime interval reducible to persistence. A cohesive spatial arrangement of persisting entities,

\footnotetext{
${ }^{58}$ Although $\mathrm{W}$ and $\mathrm{Z}$ gauge bosons are thought to have mass, the Higgs field has been postulated to account for this.
} 
mutually at rest (or thereabouts) and engaged in the ongoing exchange of force carriers, amounts to the space-filling or space-occupying associated with qualitativity. As fermions, they are mutually exclusive of each other's spatial occupancy; a property that defines the Pauli Exclusion Principle. This is in contrast to entities moving at the speed of light, which possess neither rest mass nor such exclusiveness of spaceoccupancy.

Defined as the way a property is, over and above any causal roles, quiddity has primarily been associated with time-like extension, and through the co-occurrence demonstrated above, also with rest mass. Hence, properties transmitted at less than the speed of light may be thought of as quiddistic in these terms. However, if the basic elements of the universe are, as suggested in this paper, light-like rather than timelike, and lack rest mass, then categoricity in the form of quiddity can be denied at the fundamental level.

\subsection{Structure}

In Chapter 7, I argued that since the difference between powerful and categorical dimensions is based on the issue of structure, justification is required for regarding fundamental structure as categorical rather than powerful. Alexander Bird (2005a; 2007, pp. 161-168) and Stephen Mumford (2004, p. 188) observe that classical accounts, in which spacetime is treated as 'background', have contributed to the assumption that structure is categorical. As noted in Chapter 7 of this Thesis, considering distance in Newton's Law of Gravity: $\mathrm{F}=\mathrm{G} m_{1} m_{2} / \mathrm{r}^{2}$, Mumford notes that the force could be a manifestation of spatial separation just as readily as a manifestation of the respective masses. The equation itself makes no distinction between what is categorical and what is dispositional (or powerful). Bird suggests that we intuitively derive the distinction based on classical views of space and time as 'containers', disregarding spacetime's capacity to act as an ontologically-robust agent. He notes, by way of example, conventionalisms about spacetime put forward by Mach, Poincaré, Schlick and Duhem which allow choice of geometry and metric to be conventional instead of reflecting any 'real structure' of spacetime. As Bird explains, 'If a spatial property, such as the distance between two points, is in effect the result of a conventional choice, rather than a real property of a real thing, then it is difficult to 
regard it as being active' (Bird, 2005a, p. 458). Hence, in virtue of its lack of apparent agency, distance has been considered categorical.

Background dependence leads to an assumption of a fixed theoretical structure (Kribs \& Markopoulou, 2005, p. 4) interpreted by Ellis as categorical. However, recent scientific theorising has tended to question whether spacetime is fundamental and whether it has any intrinsic metric upon which theoretical structures are 'fixed'. Rovelli points out that the distinction between background and dynamic properties collapses in General Relativity (Rovelli, 1997, p. 209), whereby the metricgravitational field carries energy and momentum, engages in causal interactions and is equivalent in terms of effect to forces such as electromagnetism. As he notes, a strong burst of gravitational waves could destroy the rock of Gibraltar as naturally as a burst of electromagnetic waves. Rovelli concludes that any ontological distinction is thus ill-founded (Rovelli, 1997, p. 193).

In keeping with Rovelli's (1997) observations, since only relative positions between dynamical physical entities are 'observable', it is meaningless to foreground physical entities with respect to some 'background'. Bird suggests that a viable physical theory, if not background free, should be at least background irrelevant (Bird, 2005a, p. 459; 2007). He argues that those interpreting space and time to be mere background should remove them from their theories, in which fundamental categorical properties ought not appear. Alternatively, theorists interpreting space and time to be more than background should characterise these as 'fully-fledged agents' and hence powerful. Either way, all 'relevant explanatory properties' should be dispositional (Bird, 2005a, p. 459). Such a stance requires spacetime to be constituted by physical entities (Relationalism), rather than a container for them (Substantivalism). ${ }^{59}$

There is much work being presently undertaken in Quantum Gravity that seeks to either present spacetime as emergent or at least to formulate theories in which it is background independent. Quantum gravity theories have been developed, for example, aiming for a background independent unification of general relativity and

\footnotetext{
59 Discussions of Ontological Structuralism, Sophisticated Substantivalism and Relationalism are outside the scope of this Thesis. However, recommendations for further reading include: Chakravartty, 2003b; Disalle, 1994; Disalle, 1995; French, 2006; French \& Ladyman, 2003; Ladyman, 1998; Pooley, 2006a, 2006b; Rickles \& French, 2006.
} 
quantum mechanics. In such models, structure is built into the universe in terms of a dynamic topology of fundamental relatedness. Loop Quantum Gravity (LQG) is one purely relational approach, pioneered by Abhay Ashtekar, Lee Smolin, Carlo Rovelli, Rolfo Gambini, Anthony Trias and others (Smolin, 2000, pp. 106-145; 2006), hypothesising 'spin networks' which can be depicted in terms of quantised units of volume comprised of edges and their intersections (or nodes) (Smolin, 1991, 1997, 2000, 2006). This portrays space not as a fundamental, categorical, persisting entity, but as a higher-order emergent of a purely relational system whose fundamental entities are quantised field-lines. ${ }^{60}$

An absence of categoricity at fundamental levels is further explored in research by Sundance O. Bilson-Thompson, Fotini Markopoulou and colleagues. Bilson-Thompson (2005) revisits 1970s pre-quark models that had conjectured quarks as being comprised of smaller components (see, the Preon Model put forward by Pati and Salam (1974), and the Rishon Model introduced by Harari and Shupe (1979)). Bilson-Thompson et al.'s Helon Model puts forward 'ribbons' (helons) as fundamental, whose topological braiding arrangements correspond to fermions. In this theory, one or two helons may represent a gauge boson, and three or more helons braided together in certain ways (i.e. topological arrangements) deliver the formation of fermions (Bilson-Thompson, 2005; Bilson-Thompson et al., 2009; BilsonThompson et al., 2007). This model does not consider certain properties such as spin and mass to be fundamental. Rather, these properties are emergent. Bilson-Thompson et al. describe the braiding model as allowing local excitations to be mapped to first generation fermions (e.g. quarks and leptons) of the Standard Model of particle physics. Like LQG, this theory posits for stable conserved quantities and fermionic entities as emergent, relying upon topological variation to supply diversity. ${ }^{61}$

\footnotetext{
${ }^{60}$ Whereas graph representations of flux lines would have edges carrying a certain amount of electric or magnetic flux, the edges of spin networks carry units of area, and the nodes represent quantized units of volume (Smolin, 2000, p. 136).

${ }^{61}$ According to this model, at least three strands of helons are required to provide an arrangement stable enough to self-sustain as a persistent fermionic object. The fermions are stable because the states that give rise to them act as 'noiseless subsystems', defined in quantum gravity by David Kribs and Fotini Markopoulou as subsystems protected from the noise of the system in virtue of symmetries (2005, p. 1). Bilson-Thompson et al. describe these as propagating coherently, protected from decoherence because they transform under symmetries that 'commute with the evolution' (2007, p. 1).
} 
The story that I present through LNA is the idea that there is a more fundamental, perhaps essentially topological, 'layer' to the universe that gives rise to spacetime and the fermionic world. This would be in keeping with Harré's suggestion that spacetime may be emergent from something more fundamental. The idea of spacetime as emergent is consistent with the claim that locations also may not be fundamental. The LNA perspective, in keeping with General Relativity, posits that absolute 'locations' do not exist in and of themselves. They are artefacts of adopting one or another coordinate system or frame of reference. Moreover, there is no such thing as a 'bare location', since every spacetime event (point-moment) has some presence of 'field'-some potential gradient involving forces-some curvature or geodesic. LNA focuses on the micro-topology of the compacted dimensions and the field fluctuations or 'forces', which invariably entail motion at the speed of light. (In this respect, they could be construed as interactions between 'temporality' and 'spatiality'.) Because the motion of field fluctuations 'takes detours' among the compacted dimensions, we get the appearance of sub-luminal motion in the form of fermionic matter. This immediately establishes frames of reference and the ability to adopt coordinate systems from which 'location' emerges. Prior to frames of reference, spacetime's geometry cannot even be defined. However, any apparent sub-luminal motion is not that of any particular. It is just a relation between certain geodesics of open spacetime and certain geodesics of the micro-topology, which all go to constituting the field. I am treating such relations as powerful, in terms of their being descriptions of the nature of their relata.

The importance of the above is that, whether one chooses to consider spacetime as background or more than background, there is no transparent reason why the structure presented by the dimensional topology should be considered categorical. LNA proposes that spacetime is constituted by gauge bosons (field fluctuations) and a specific dimensional topology, neither of which may be viewed as categorical or qualitative. The categorical has been defined in terms of being 'actual' in the sense of being ontologically-robust, non-powerful, exhausted in its manifestation, and individuated or self-contained; whereas the dispositional is often characterised as representing possibility, capacity or an ontological status that is somehow non-robust, powerful (dynamic or active), and non-individuated in the sense of 'stretching out' 
beyond itself. LNA's conjectured micro-topology adheres to neither of the delineations above. Rather, it can be viewed as both ontologically-robust and powerful. The interdependence of the field's potential and its topology also supports an interpretation of the topology being, like Harré's Field, an intensive rather than an extensive distribution. Thus, it is not bordering off, or constraining or self-contained in the way supposed of the categorical.

As already noted, Bird considers that spacetime structure, since not justifiably categorical, should be considered dispositional. However, I argue that dispositionality should not be treated as the default to being non-categoricity. Perhaps we should distinguish between the terms 'powerful' and 'dispositional' such that the notions of 'potentiality', and hence 'non-ontologically robust', as applied to dispositionality, should not be applied to the term 'powerful'. Thus far I have argued that the qualitative does not appear at the very basic level of the universe, in the form of either haecceity or quiddity. Denying these at the basic level is consistent with regarding the geometric/topological structure as non-categorical. I am suggesting that all that exists are the effects of fluctuations of the field, which are intrinsically light-like, corresponding to pure power. However, this entails no unmanifested power at the fundamental level, and thus no room for dispositionality in the sense of unmanifested potentiality, although differentiation and hence context-dependent modality may obtain at emergent levels. Thus, denial of categoricity at fundamental levels, while equating to a pure-power ontology, does not entail the fundamental being purely dispositional, if by this we mean a world comprised of pure possibility.

We have an overwhelming sense of the world as comprising 'things' standing in relation to each other spatially, and it seems that we derive this sensation from the properties of things that are temporally extended. Yet, without the power or ability of things to be perceived - to affect us in the ways that they do-we would lack the capacity to sense this ostensible spatial primacy of the world. LNA tells a story of how we might get from a homogeneous pure-power base to the emergence of nonhomogeneity without introducing categorical properties or fundamental particularity into the ontology. But this entails that what we have traditionally taken to be differences between property types, e.g. dispositional versus categorical properties, are only higher-order artefacts of the action of pure power. In Chapter 11 of this 
Thesis, I criticised the Identity Theory of Properties in terms of its attempt to identify dispositional and qualitative properties as a single 'power-quality'. Part of that criticism derived from Charlie Martin and John Heil characterising two seemingly unique natures to create their power-quality, while criticising property-monist theories for lacking an important component supplied by either the qualitative or the dispositional. LNA differs from the Identity Theory of Properties in that my claim is not to collapse the two types of property together. Rather, I suggest that in terms of fundamental ontology, we should disengage from the concepts of dispositionality and qualitativity (or categoricity) altogether, and accept that the descriptions they satisfy only have meaning at higher levels.

Although Graham Nerlich's account (1993a, 1993b) differs from that of Harré et al. and from LNA in that it views spacetime as fundamental, it is nonetheless useful as an illustration for how fundamental structure might be considered non-categorical, and yet not dispositional in the sense of pure potentiality. Nerlich calls his fundamental a 'unique ontic category'; or the 'concrete immaterial'. He supposes that since the field itself is structured-and thereby operates to alter the direction of anything passing through it, according to its intrinsic geodesic structure-spacetime curvature determines. On one hand, Nerlich's spacetime is structure. This would suggest, according to certain notions of structure, such as Brian Ellis's categorical dimensions (Ellis, 2001b, 2002, 2008b), that spacetime is categorical. On the other hand, Nerlich's spacetime 'acts' determinately, affording it the status of being not only ontologically-robust, but potent. Thus Nerlich's spacetime is not categorical, if by the term 'categorical' we mean 'non-power' or even 'independent of power'. Neither can spacetime, as the single substratum, be called categorical in the sense of being some distinct category of thing, since at least two entities are required for such a distinction to be made. Thus, Nerlich's narrative lends itself to the claim for the cogency of structure of pure potency, notwithstanding that spacetime is ontologicallyrobust (actual). Thus it conforms to descriptions of neither the categorical nor the dispositional, but can be described as simply 'powerful'.

Consistency demands that if there is nothing categorical at the fundamental level, there can be nothing ontologically categorical at higher levels. LNA describes how the fermions that give rise to the manifest world might emerge from a pure power 
basis, and with these entities we get the possibility of differentiation (but not strict distinction) between 'objects', or more precisely 'clumps in the field'. Such differentiation allows for the attribution of location, size, shape and other properties that are viewed as qualitative, as well as the emergence of higher-order powerful properties. Hence, at this level, does the dispositional-categorical dichotomy appear to emerge, along with the appearance of contingency and possibility. However, it does not follow from the effects of non-homogeneity at higher levels that the world is nonhomogeneous at the fundamental level. Neither does it follow that our familiarity with contingency and possibility at higher levels requires them to be ontologically-robust.

One concern might be expressed in that LNA represents basic fermions, like quarks, as circulations of gauge bosons, whereby any given circulation remains associated with a particular location in some inertial frame of everyday space - the point of intersection between macroscopic and microscopic dimensions where the relevant change of trajectory occurs. But how does LNA get from a mere locus of trajectory changes to more complex yet stable configurations, such as a proton? By way of an answer, consider the following description of dimensionality in terms of allowing or restricting angular momentum. Given some point-moment event $\mathrm{X}$ and some quantity of time $\mathrm{T}$, there are just two events existing at the same point as $\mathrm{X}$, and which are separated from it by $\mathrm{T}$. These two events are located at $\mathrm{T}$ in the future from $\mathrm{X}$ and at $\mathrm{T}$ in the past from $\mathrm{X}$. However, given some point-moment event $\mathrm{X}$ and some quantity of space $\mathrm{S}$, there are many events existing at the same moment as $\mathrm{X}$, and which are separated from it by S. These many events are located at all the points forming a sphere of radius $\mathrm{S}$ around $\mathrm{X}$.

Schematically, in the latter case, $\mathrm{X}$ is surrounded by an array of events which are simultaneous and may be equi-spatial also from each other. Consequently, in the context of fundamental particles, we have the potential for a secondary or higherorder 'one-way circulation' among the events equi-spatial from X. Analogous to a circular-driveway, this is available in two or more dimensions that supply an angular metric, but there is no prospect for any similar circulation among the merely two events equi-temporal from $\mathrm{X}$, which exist at the same point as $\mathrm{X}$. (One dimension allows for only a linear network, much like a drive-in-back-out-driveway.) Subject to scientific interpretation, and in keeping with the description of LNA in sections 14.1 
and 14.2, what is circulating in this higher-order network would be suitable gauge bosons. The events constituting the array that surrounds event $\mathrm{X}$ would be those at which the gauge boson paths 'inside' each quark (say) intersect with uncompacted 3D space, allowing the quarks to exchange gauge bosons. Our categorical-dispositional distinction may emerge from the dimensional asymmetry of spacetime which facilitates the formation of 'matter' constituted by the exchange of gauge bosons. Continually doing so across sufficiently short separations (in connection with Heisenberg's Uncertainty Principle ${ }^{62}$ ), we may suppose these complex entities to continuously 'self-replicate'. ${ }^{63}$ Concrete particles would thus arise as described earlier. Taking curled-up extra dimensions into account, the sizes, shapes and orientations of objects are all ultimately defined by changes of trajectory of field fluctuations, which just are their effects upon each other.

The upshot of the 3-to-1 dimensional asymmetry is that such more complex entities may exist only as persisting spatial arrangements of conserved quantities. They appear as stuff that extends 'gratuitously' through time, upon which entropy imposes a well-defined direction. But we too are such spatially confined networks. As a result, we readily perceive motion-through-space, but not motion-through-time (faster than light). We are 'primed' by expediency to perceptually encounter the world in terms of the velocities of other particle-like networks, giving rise to the intuition of spatial primacy. The 3-to-1 asymmetry is thus translated into a bias that favours identifying the constituent world as 'substantial' or qualitative, with substance considered to be spatially oriented. Hence, we are incidentally yet inescapably disposed to identify the world's contents primarily in terms of spatial arrangements of events. This presents an intuitively forceful, yet ultimately illusory, distinction of ontological status between arrangements of events in space and those in time. The former tend to be called 'qualitative', and the latter 'dispositional'. Although derived from the prior 3-to-1 asymmetry, however, the distinction is essentially an artefact of properties corresponding to perception. Whenever it seems to manifest in 'spaceonly', power appears qualitative. Although power manifests always in spacetime, we

\footnotetext{
${ }^{62} \Delta \rho \times \Delta s \leq$ Planck's constant. Shorter separations provide for greater uncertainty in momentum and other quantities, perhaps contributing to network stability.

${ }^{63}$ This is reminiscent of David Armstrong's 'immanent causation' (1997).
} 
arrive at the false impression that mere space-filling entails 'substance'. This is expressed in relation to the appearance of persistent, distinct objects, and arises because everyday space, in comprising three dimensions, seems more primary than time. Objects apparently 'sit still' in space and persist through time, occupying spatial regions in an 'orthogonally extended' fashion, whereas no analogous physical process involves sitting still in time.

A flow-on observation is central to the main themes of this thesis, harking back to Shoemaker's Causal Theory of Properties. As pointed out by Chakravartty and others, causal chains of effect need never have point-moments at which they begin or end. Correspondingly, LNA proposes that, between being absorbed and emitted by fermions, gauge bosons might merely circulate among compacted orthogonal spaces. Indeed, the gauge boson itself represents a causal chain with neither beginning nor end. At the level of fermions and higher orders of manifestation, however, we assign primary ontological status to apparently distinct objects, including ourselves. This behaviour inclines us to interpret them as the spatial endpoints of causal chains, and naturally reinforces the interpretation of events as Humean independent - in line with the false intuition that spatial arrangements of events possess categoricity.

I noted earlier that if there is nothing categorical at fundamental levels, then ontologically-robust qualitativity at higher levels would be inconsistent. Instead, we are afforded an intuitively forceful impression of the qualitativity that arises by virtue of our own involvement as very complex regions of a fundamentally powerful field which is dimensionally asymmetric. Since this picture rules out 'substance' in terms of spatially-oriented particulars, bearers of power or categorical properties, how can we rescue our commonsense notion of substance in power terms? Can the substance role be played by 'force', if the structure of spacetime is 'intensive' rather than 'extensive' in nature, and thereby accounted for as power?

Returning to Robinson's marks of substance, discussed in section 13.3, it is possible to satisfy (i) in providing something which is ontologically basic; namely, a fundamental pure-power field capable of affecting and being affected. In terms of mark (ii) — substance being relatively independent and durable_-LNA describes a Foundation-Monism that absolutely fulfils this description. Mark (iii) requires that 
substance be the subject of predication and the bearers of properties. In the same fashion that Harré's Great Field only partially fulfils this mark, so LNA is formulated as a Bundle Theory whose properties are higher-order manifestations of fundamental structure, although the structure I have in mind is powerful. Mark (iv) requires that substance be the subject of change. Again, in keeping with Harré's pure-power basis, LNA considers field fluctuations to be immutable, although the fermionic entities that emerge are consistent with changeable Aristotelian individuals described by Harré et al. The fifth, six and seventh marks provided by Robinsons do not fit within the framework of LNA, since these assume distinct objects and the requirement for individuation, assumptions axiomatically denied for LNA. The fermionic building blocks of the manifestly qualitative world are described by these marks, but I account for these as emergent, and for spatially-oriented ostensible substance in terms of dimensional asymmetry. Mark (viii) is very relevant to the ontological system described by LNA, since what is crucial to the system is precisely the fundamental, powerful field. In terms of these marks of substance, therefore, a satisfactory account of the substance role is provided in terms of structure that is fundamentally powerful.

\subsection{Summary}

This Chapter rises to the challenge of providing a sustainable account of the manifestly qualitative world by describing how ostensibly qualitative objects might arise in terms of complex composites of fundamental field fluctuations interacting throughout compacted-dimensional orthogonalities. Spacetime, locations, fermions (conforming to the Pauli Exclusion Principle, thus contributing to the spatial extension of objects), the categorical-dispositional distinction and notions of possibility; all arise at higher levels. The challenge for LNA is to describe how we get from the fundamental to these higher levels. I have conjectured that the ostensible spatial priority of the world might be thought to obtain by virtue of: first, the 'barber poles' or micro-topological networks giving rise to massy and persistent 'entities', comprised of circulating gauge bosons which travel at the speed of light, possess no spatiotemporal extension, and lack rest mass. The networks conserve physical quantities (such as mass and charge), absorbing and emitting both occasionally real and continually 'virtual' gauge bosons. Second, spacetime's numerical asymmetry- 
several spatial dimensions versus one time dimension-permits things to sit still in space but not in time, affording our different attributions of ontological status. The objects of the world, including ourselves, are very complex barber poles, ultimately comprised of pure power and subsisting within a fundamentally powerful structure that reflects Harré's Great Field. 



\section{CHAPTER 15}

\section{SUMMARY AND CONCLUSION}

A central aim of this Thesis involved determining what fundamental entities would suffice to account for the manifest world. Much of the discussion about fundamental entities revolves around the distinction between dispositional and categorical properties, and the nature of their bearers. This Thesis has critically examined various representational stances on these issues in providing a comparative analysis of the competing positions.

Section 1 outlined and discussed David Armstrong's Categorical Monism, according to which all properties are categorical. Criticisms put forward by Charlie Martin, Herbert Hochberg and Alexander Bird were employed to argue that this stance faced the problem of satisfactorily accounting for the necessity required of 'strong' causality, an argument that holds whether Armstrong's laws are considered to exist contingently or necessarily. If the laws of nature are contingent, they must be external to the property instances that they relate. In this case, nomic regularity between instances requires an explanation derived other than by these laws. To this end, Armstrong proposes higher-order laws — relations between types of propertiesto represent the necessitating relation, $\mathrm{N}$, responsible for ensuring regularities between the instances. This set-up is problematic, since Armstrong does not allow higher-order types to exist independent of their instances. Thus, still higher-order types are required to explain these, and so on. It turns out that the theory requires higher- and higher-order types to explain the necessitating relations of each subsequent level, but short of allowing an end-point such as some transcendent Platonic Ideal, cannot ultimately provide a source of necessity. Necessary laws could, in theory, solve this problem. However, such a move would introduce irreducible dispositionality, thus rendering it no longer Property Monist.

Section 2 outlined and discussed Sydney Shoemaker's Causal Theory of Properties. Its reductionist formulation of power and properties is criticised by Richard Swinburne and others insofar as pure-power ontologies allegedly lack means of identifying qualitative properties, such as shape and size, involved in perceiving the 
objects of the manifest world. George Molnar points out that such arguments are flawed to assume that such qualitative properties exist at fundamental levels. Instead, his criticism of Shoemaker focuses on the criteria by which properties are identified as genuine rather than ontologically eliminable. Using spatiotemporal relations as a counterexample to Shoemaker's formulation, Molnar first argues that properties and powers are independent. His S-property account then opens the way to claim a causal role for spatiotemporal properties and relations in moderating the intrinsic powers of their bearers. Since spatiotemporal properties are not intrinsic, they repudiate Shoemaker's tenet that only intrinsic properties are causally contributing.

The status of Shoemaker's spatiotemporal relations are also implicated in the issue of distinctness between entities. According to the Principle of Independence, for theories that postulate distinct objects, relations between such objects must exist contingently, hence self-contained and categorical. Yet, the Causal Theory of Properties requires these relations to be supervenient upon pure-power properties. If the relata ground the relations as Shoemaker supposes, then an explanation of distinct relata 'linking' together would have to be given in terms of the properties of the relata themselves. To completely specify circumstances for the manifestation of power, every object in the universe is involved. Moreover, since all genuine properties are causally contributing, if Molnar's S-property argument holds, then all spatiotemporal relations should be seen as genuine properties and, by Shoemaker's criteria, intrinsic to their bearers. Indeed, if spatiotemporal relations were intrinsic, they would be intrinsic to the system representing the inclusion of all of their relata. The result is that the whole universe must be considered in order to fully specify any particular relation between its object-contents, resulting in an underdetermination of local, extrinsic relations.

Redressing the problems incurred by reduction of powers to properties, and vice versa, requires that Shoemaker reformulate his theory in terms of either removing the reduction between powers and properties or crafting a Foundation-Monism. $\mathrm{He}$ chooses the former option, presented in his later revision. I have argued that this course removes the theory from a pure-power view in favour of incorporating quiddity and hence fundamental categoricity. 
What is shown by the above, is that if an ontology attempts a description of fundamentality in terms of the categorical-dispositional distinction, rectifying the shortfalls of both Monist accounts appears to require adding the additional property type; irreducible dispositionality in the case of Categoricalism, and quiddity in the case of the Causal Theory of Properties. Section 3 provided a coverage of issues faced by Property Compatibilist theories, which aim to avoid the concerns of the Propertymonist stances by embracing both dispositional and categorical fundamental properties. Stephen Mumford notes that dualist claims involve a 'real division' of reality between two differently-natured properties (1998, p. 95). We have an overwhelming sense of the world as being spatially-oriented, and it seems that we derive this sensation from the properties of things. Yet, we are aware that without the power or ability of things to be perceived - to affect us in the ways that they do-we would lack the capacity to sense this ostensible spatial primacy of the world in which we live. In attempting to capture both these aspects of our reality, Property Compatibilists builds them in at the fundamental level in one way or another.

The New Essentialism of Brian Ellis, the focus of Chapters 6 and 7, is a notable dualist account that incorporates both dispositional and categorical fundamental properties. Its categorical modulators or dimensions, such as spatiotemporal relations, play a causal role in the operation of powers. Ellis bases the mutual-exclusion of dispositional and categorical properties upon the premise that categorical properties are 'structural' and dispositional properties are 'non-structural'. Moreover, although both play a causal role, dispositional properties can be considered to be causal powers, whereas categorical properties are described as independent of these powers.

This raises the question of whether New Essentialism could feasibly replace its fundamental dimensions with an alternative version of structure that is powerful, rather than categorical. Three reasons for making such a change were discussed. First, it would avoid the problem of how to forge an identity of categorical dimensions in terms of quiddity, while also allowing them a causal role. Second, structure that is powerful would remove problems associated with positing necessary laws. And third, it allows the dimensions to be observed via their effects without suffering the problem of how they are to be observed, an issue that arises if the essential nature of 
categorical dimensions is quiddistic. In subsequent Chapters, I followed up on the suggestion for fundamental, powerful structure by suggesting that the categoricaldispositional distinction arises only at higher levels; and that the fundamental level is comprised of pure power that defies description as either categorical or dispositional.

In Chapters 8 and 9, I briefly outlined and discussed Ullin Place's Conceptualism, a view that considers categorical and dispositional properties to be different types of entity, although fundamentally inter-dependent. Place describes the categorical in terms of being 'actual' and 'present', and the dispositional in terms of past counterfactual and possible future considerations. Place adheres to the Humean Principle of Independence in adopting the view that dispositions are distinct from their associated microstructure, which causes them to exist. In Chapter 9, I discussed two objections that have been put forward: the first, raised by Armstrong, concerned the inability for Place to provide more than a counterfactual truthmaker for ascribing dispositions; and the second, by Mumford, concerned a regress that obtains because dispositions are caused by the microstructures of the objects that possess them, as well as partially comprising them. Each level of microstructure has dispositional properties, but these must be caused by still more basic structures, so the regress would bottom out only with (uncaused) irreducible dispositional properties.

The Identity Theory of Properties was the focus of Chapters 10 and 11. I concluded that Martin's and Heil's theory faces difficulties that resemble, in principle, those which other dualist theories encounter; its theoretical assumptions being informed by the dichotomy that underpins the concepts of the qualitative and dispositional. Their strict identity is incompatible with the assertion that both make unique contributions to the world, which would imply some ontologically-robust difference between the two. Attempts at resolving this contradiction, by proposing epistemological, context-dependent or functional-role explanations, fail because no antecedent is available to warrant differently considering the dispositional and categorical. I argued that Heil's theory falters in trying to unite the qualitative and powerful by positing power to be non-relational. Power-qualities and pure-qualities differ in terms of how they may bestow power, yet Heil offers no explicit account of this action of bestowing.

Section 3 concluded that attempts to posit both categorical and dispositional 
properties at fundamental levels encounter serious problems with explaining how they might differ in nature, yet work together. Endowing them with unique natures, such that they represent distinct property types, leaves open the question of how purely quiddistic properties would be detected, and what function they would serve. Any role would seem inextricably tied to producing effects, and thus be powerful. Perspectives that intrinsically combine, identify or describe the two raises semantic questions concerning their duality.

The final part of this Thesis, Section 4, discusses Field Theory as a viable alternative to world-views that hold distinct objects as fundamental. The Foundational-Monist stance that I discussed in Chapter 14 was largely based on Rom Harré's and Edward H. Madden's Theory of Causal Powers, particularly with respect to their view of a single 'Great Field' comprised of pure-power Parmenidean 'Individuals'. Harré endeavours to justify the notion of pure power by first venturing the principle that only change needs explanation. On this basis, a suitably ultimate fundamental entity, not subject to change, demands none. Harré and Madden's fieldtheoretic view has much to offer in terms of accounting for strong causation via enduring, underlying generative mechanisms ultimately comprised of their explanans - the Parmenidean individual—responsible for causal regularities. The sole, primitive entity in this monist ontology is the Great Field, characterised as an intensive distribution of different magnitudes of potentiality, represented in terms of overlapping contours and giving rise to the spacetime metric. The individuality of objects manifested to us is explained in terms of clusters of Parmenidean Individuals - singularities whose gradients vary sharply from their surroundsalthough the Parmenidean Individuals are not qualitatively distinct.

Chapter 13 served as an introduction to the Foundational-Monist view that I subsequently developed in Chapter 14. In Chapter 13, I defended a worldview according to which the fundamental entity is comprised of pure power-understood in Harré's terminology as 'Influence'-best characterised differently from traditional accounts of either categoricity or dispositionality. I distinguished a purely dispositional world-dispositionality understood in terms of ability, possibility, capacity or potentiality - from one that is fundamentally powerful. I also argued why the categorical, in terms of quiddity, self-containment and association with contingent 
laws of nature, is not a fitting characterisation of fundamental power, and argued that the categorical-dispositional distinction should be regarded as emerging only as a higher-order phenomenon, once 'clumpiness' emerges in the form of fermionic entities. How this may be conjectured to occur is described more fully in Chapter 14, in the light-like network account (LNA) that I have put forward.

Throughout Chapters 13 and 14, I draw a very close parallel between the Great Field of Rom Harré and my own Foundational-Monist position. Since a pure-power theorist rejects the requirement for power to be grounded in the categorical at fundamental levels, she faces the challenge of explaining the ostensible qualitativity of the manifest world. Chapter 14 rose to this task by providing an account of the world starting from a base devoid of categorical or qualitative properties or entities. It explored the possibility of a 'field' whose fluctuations are force-carrying entities - the gauge boson types - differentiated in terms of their relation to several or more curledup spatial dimensions. Such 'Calabi-Yau spaces' are widely conjectured to constitute a built-in micro-topology at every point in macroscopic space. Field fluctuations might thus 'traverse' more than the three everyday spatial dimensions. Although penetrating this extra-dimensional labyrinth, they would constantly end up at the same (inertial) location within everyday space. However, the characteristics of the process would depend on the gauge boson type, corresponding to which dimensions were involved in any fluctuation. Just as Harré's Parmenidean Individuals comprise the Great Field, so these gauge bosons comprise the micro-topology as well as the intensive distribution of power which gives rise to spacetime, emerging from the light-like fluctuations that continuously 'flow through' the field. (The sense of the term 'through' here, is meant as a wave flows through water, rather than as a fish swims 'through' it.) Via this approach, and subject to further scientific analysis, LNA represents a description of how the ostensibly categorical objects of the manifest world might arise in terms of complex composites of fundamental field fluctuations interacting with respect to the compacted dimensions.

There are some differences postulated between Harré's Parmenidean Individuals and the neo-Parmenidean entities of LNA. The former are singularities in the field, obtaining their pseudo-individuality in terms of a steep gradient of potential magnitude that marks off their field region. LNA's gauge bosons, however, are field 
fluctuations that engage in quantum interactions, being absorbed and emitted by circulation networks; hence they are quantised with respect to such networks, which conserve physical quantities (such as mass, charge, colour and isospin). When not being absorbed or emitted, their wavelike character endows them as indeterministic in terms of spatiotemporal extension, although influential in terms of potentiality.

Unlike Harré's characterisation of Parmenidean Individuals as singularities that that persist through time, I stress an even-handed treatment of gauge bosons in terms of space and time. Because they are light-like, given any two events, the amount of space between them equates to the amount of time between them. That is to say, these field fluctuations are effectively one-dimensional interactions of time and space. Just as they possess no purely spatial extension, neither do they posses purely temporal extension. They can not 'sit still' in space while persisting in time, as the Parmenidean singularities appear to do. Moreover, since they represent no purely spatial extension, yet they engage directly in causal 'effect' or 'Influence', they defy description as categorical - in terms of representing the Lockean primary properties such as size and shape, or providing the 'what' or 'shell' as Richard Swinburne, Charlie Martin and others claim—but represent pure power. This claim undermines arguments, put forward by proponents of Swinburne's regress, that a viable ontology requires primitive categoricity at the fundamental level.

Chapter 14 puts forward the notion that the ostensible spatial priority of the world is obtained by virtue of: first, the 'barber poles' or micro-topological networks comprised of circulating gauge bosons (which travel at the speed of light and which cannot be said to represent purely spatial or purely temporal extension). Second, macroscopic spacetime's numerical asymmetry-several spatial dimensions versus one time dimension - favours objects as complexes of time-like (hence, persisting) rather than space-like processes. Since we are also complex conglomerations of such networks and resultant emergent entities, we view the world in terms of things that move about in space, perceiving these as persisting causal agent or 'objects'. Hence, things that are time-like afford our attributions of the ontological status of the world as substantial. This Thesis argues, however, that this end-point can be attained by beginning with nothing but pure power. A further conclusion is that causal chains of effect actually have no ending point-moments; just as, between being absorbed and 
emitted by fermions, gauge bosons might merely circulate among compacted orthogonal spaces. At this and higher orders of manifestation, however, our attribution of primary ontological status to apparently distinct objects, including ourselves, inclines us to perceive them as the spatial endpoints of causal chains of effects. This naturally sustains the view that events possess Humean independence, and that spatially arranged substance has categorical properties.

In conclusion, I entertain an idea of the universe, at base, as a fluctuating field comprised of and comprising pure power, neither borne by a categorical entity nor grounded in categorical properties. If the basic ingredients of our world were lightlike force carriers (or conceivably 'bits of force') with neither purely spatial nor purely temporal extension, nor bordered, then these fulfill the criteria of pure power. However, fundamental power does not, in the view taken here, qualify as dispositional, if the term 'dispositional' is taken to refer to possibility, capacity or unmanifested potentiality. Rather, the Foundation-Monist perspective expressed in this Thesis treats spacetime as emergent from a basis of fundamental power that cannot be described as either categorical or dispositional.

\section{REFERENCES}

Aitchison, I.J.R. (1991). The Vacuum and Unification. In S. Saunders \& H. R. Brown (Eds), The Philosophy of Vacuum (pp. 159-95). Oxford: Clarendon Press.

Aquinas (1270/1948). Summa Theologica: Question 2, Article 3, translated by Fathers of the English Dominican Province In St. Thomas Aquinas Summa Theoligica: Complete Edition in Three Volumes (pp. 2576-77 ). London: Burns and Oates.

Aristotle (1952). Metaphysics III: Book Beta. In R. Hope (Ed.), Aristotle Metaphysics. New York: Columbia University Press.

— (1960). Metaphysics. New York: The University of Michigan Press.

Aristotle, Cooke, H.P. \& Tredennick, H. (1938). The Categories On interpretation Prior Analytics. Cambridge, Mass.: Harvard University Press; London: Heinemann. 
Armstrong, D.M. (1961). Perception and the Physical World. New York: Humanities Press.

— (1966). The Nature of Mind. Arts (Proceedings of The Sydney University Arts Association), 3, 37-58.

_ (1968). A Materialist Theory of the Mind. London: Routledge \& Kegan Paul.

_ (1972). Materialism, Properties and Predicates. Monist, 56 (April), 163-76.

_ (1978a). Universals and Scientific Realism: A Theory of Universals, Vol. II, Cambridge; New York: Cambridge University Press.

- (1978b). Universals and Scientific Realism: Nominalism and Realism, Vol. I, Cambridge: Cambridge University Press.

- (1980). The Nature of Mind. In The Nature of Mind and Other Essays (pp. 115). Brisbane: University of Queensland Press.

_ (1983a). Recent Work on the Relation of Mind and Brain. In G. Floistad (Ed.), Contemporary Philosophy: A New Survey (pp. 45-79). The Hague: Nijhoff.

_ (1983b). What Is a Law of Nature. Cambridge: Cambridge University Press.

— (1984a). Consciousness and Causality. In D.M. Armstrong \& N. Malcolm (Eds), Consciousness and Causality. Oxford: Basil Blackwell.

— (1984b). Self-Profile. In R.J. Bogdan (Ed.), D M Armstrong (pp. 2-51). Boston: Reidel.

— (1989). A Combinatorial Theory of Possibility. New York: Cambridge University Press.

_ (1996a). Dispositions as Categorical States. In D.M. Armstrong, U.T. Place, C.B. Martin \& T. Crane (Eds), Dispositions: A Debate (pp. 15-18). New York: Routledge.

- (1996b). Place and Armstrong's Views Compared. In D.M. Armstrong, U.T. Place, C.B. Martin \& T. Crane (Eds), Dispositions: A Debate (pp. 33-48). New York: Routledge.

— (1996c). Reply to Martin. In D.M. Armstrong, U.T. Place, C.B. Martin \& T. Crane (Eds), Dispositions: A Debate (pp. 88-104). New York: Routledge.

— (1997). A World of States of Affairs. Cambridge; New York: Cambridge University Press. 
— (2000). The Causal Theory of Properties: Properties according to Shoemaker, Ellis and Others. Metaphysica, 1 (1), 5-20.

— (2001). Two Problems for Essentialism. In Australasian Association for the History, Philosophy and Social Studies of Science. Melbourne: Reprinted in Brian Ellis (2002). The Philosophy of Nature: A Guide to the New Essentialism. Chesham: Acumen. Appendix, pp. 167-171.

— (2004a). How Do Particulars Stand to Universals? In D.W. Zimmerman (Ed.), Oxford Studies in Metaphysics (Vol. I, pp. 139-54). Oxford: Clarendon Press.

- (2004b). Truth and Truthmakers. Cambridge: Cambridge University Press.

— (2005a). Four Disputes about Properties. Synthese, 144, 309-20.

— (2005b). Reply to Bird. Analysis, 65 (3), 264-65.

- (2005c). Reply to Simons and Mumford. Australasian Journal of Philosophy, 83 (2), 271-76.

- (2006). Reply to Rissler. Australasian Journal of Philosophy, 84 (2), 211-12.

Baxter, D.L.M. (2001). Instantiation as Partial Identity. Australasian Journal of Philosophy, 79 (4), 449-64.

Bickhard, M. H. (accessed 2008). Quantum Level Variations and Selections. Retrieved 2009 March 03, 2008, Online at http://www.lehigh.edu/ mhb0/ quantumvarsel.html.

Bigelow, J. \& Pargetter, R. (1999). Critical Notice - Book Review. Canadian Journal of Philosophy, 29 (4), 619-33.

Bilson-Thompson, S.O. (2005). A topological model of composite preons [Electronic Version]. Retrieved, 30 March, 2009. Online at: http://arxiv.org/abs/hep$\mathrm{tj} / 0603022 \mathrm{v} 2$

Bilson-Thompson, S.O., Hackett, J. \& Kauffman, L.H. (2009). Particle Topology, Braids and Braided Belts [Electronic Version] Classical and Quantum Gravity. Retrieved, 30 March, 2009. Online at: http://arxiv.org/abs/hep-tj/0603022v2

Bilson-Thompson, S.O., Markopoulou, F. \& Smolin, L. (2007). Quantum Gravity and the Standard Model, Classical and Quantum Gravity: IOP Publishing

Bird, A. (1998). Dispositions and Antidotes. Philosophical Quarterly, 48 (191), 22734. 
— (2003). Structural Properties. In H. Lillehammer, G. Rodríguez Pereyra \& D.H. Mellor (Eds), Real Metaphysics: Essays in Honour of D.H. Mellor (pp. 154-68). London; New York: Routledge.

— (2005a). Laws and Essences. Ratio (new series), XVIII (4), 437-61.

— (2005b). The Ultimate Argument Against Armstrong's Contingent Necessitation View of Laws. Analysis, 65 (2), 147-55.

— (2005c). Unexpected A Posteriori Necessary Laws of Nature. Australasian Journal of Philosophy, 83 (4), 533-48.

— (2006). Kinds and Essences. In A. Drewery (Ed.), Metaphysics (pp. 63-87). Malden, MA; Oxford; Victoria, Australia: Blackwell Publishing.

— (2007). Nature's Metaphysics: Laws and Properties. Oxford: Oxford University Press.

Biswas, T. (2003). Symmetry Breaking of Guage Theories via Internal Space dynamics. Retrieved March 6, 2009, Online at http://jhep.sissa.it/archive/ papers/jhep022003054/jhep022003054.pdf

Black, R. (2000). Against Quidditism. Australasian Journal of Philosophy, 78 (1), 87 104.

Blackburn, S. (1990). Filling in Space. Analysis, 61, 267-74.

Bohm, D. (1980). Wholeness and the Implicate Order (1983). London, Boston, Melbourne and Henley: ARK Paperbacks.

— (1987). Hidden Variables and the Implicate Order. In B.J. Hiley \& R.D. Peat (Eds), Quantum Implications: Essays in Honour of David Bohm (pp. 33-45). London \& New York: Routledge \& Kegan Paul.

Boler, J. F. (1963). Charles Pierce and Scholastic Realism. Seattle: University of Washington Press.

Boscovich, R. (1922). Theoria Philosophiae Vaturalis. Venice, 1763 (A Theory of Natural Philosophy) New York: M.I.T. Press.

Bradley, F. H. (1897). Appearance and Reality. Oxford: Clarendon Press.

Campbell, K. (1976). Metaphysics: An Introduction. Encino, California: Dickenson Publishing.

— (1990). Abstract Particulars. Oxford, UK; Cambridge, Mass.: Blackwell.

Carr, B. (1978). Review. Mind, 87 (346), 305-06. 
Caves, C.M., Fuchs, C.A. \& Schack, R. (2008). Subjective Probability and Quantum Certainty [Electronic Version] Online at: arXiv:quant-ph/0608190v2 </abs/ quant-ph/0608190v2>

Chakravartty, A. (2003). The Dispositional Essentialist View of Properties and Laws. International Journal of Philosophical Studes, 11 (4), 393-413.

Choi, S. (2003). Improving Bird's Antidotes. Australasian Journal of Philosophy, 81 (4), 573-80.

— (2005). Do Categorical Ascriptions Entail Counterfactual Conditionals? The Philosophical Quarterly, 55 (220), 495-503.

— (2006). The Simple Vs. Reformed Conditional Analysis of Dispositions. Synthese, 148, 369-79.

Cohen, B. \& Madden, E.H. (1973). Harre and Nonlogical Necessity. The British Journal for the Philosophy of Science, 24 (2), 176-82.

Crane, T. (1996). Introduction. In D.M. Armstrong, U.T. Place, C.B. Martin \& T. Crane (Eds), Dispositions: A Debate (pp. 1-11). London; New York: Routledge.

Crane, T., Armstrong, D. M., Martin, C. B. \& Place, U. T. (Eds) (1996). Dispositions A Debate: D.M. Armstrong, C.B. Martin and U.T. Place. New York: Routledge.

Davies, P. \& Gribbin, J. (1992). The Matter Myth. London: Penguin Group.

Dipert, R R. (1997). The Mathematical Structure of the World: The World as Graph. Journal of Philosophy 94, 329-58.

Dodd, J.E. (1984). The Ideas of Particle Physics: An Introduction for Scientists. Cambridge: Cambridge University Press.

Dreske, F.I. (1977). Laws of Nature. Philosophy of Science, 44, 248-68.

Drewery, A. (Ed.) (2006). Metaphysics. Malden, MA; Oxford; Victoria, Australia: Blackwell Publishing.

Earman, J. (1970). Space-Time, or How to Solve Philosophic Problems and Dissolve Philosophic Muddles Without Even Trying. The Journal of Philosophy, 67, 259-77.

Earman, J., Glymour, C. \& Stachel, J. (Eds) (1977). Foundations of Space-Time Theories. Minneapolis: University of Minnesota Press. 
Ehring, D. (2004). Distinguishing Universals from Particulars. Analysis, 64 (4), 32632.

Einstein, A. (1934). Clerk Maxwell's Influence. In Essays in Science (English Translation ed., pp. 40-45).

— (1998 [1905]). On a Heuristic Point of View Concerning the Production and Transformation of Light. In J. Stachel (Ed.), Einstein's Miraculous Year. English translation of the 1905 paper, 'Über einen die Erzeugung und Verwandlung des Lichtes betreffenden heuristischen Gesichtspunkt', Annalen der Physik 17:132-148. Princeton, N.J.: Princeton University Press.

Ellis, B.D. (1999). Causal Powers and Laws of Nature. In H. Sankey (Ed.), Causation and Laws of Nature (pp. 19-34). Dordrecht, The Netherlands: Klewer Academic Press.

- (2000). Causal Laws and Singular Causation. Philosophy and Phenomenological Research, 61 (2), 329-51.

- (2001a). Response to Armstrong. Paper presented at The Annual Conference of the Australasian Association for the History, Philosophy and Social Studies of Science, Melbourne. In The Philosophy of Nature: A Guide to the New Essentialism (pp. 171-76). Ithaca: McGill-Queen's University Press.

— (2001b). Scientific Essentialism. Cambridge: Cambridge University Press.

— (2002). The Philosophy of Nature: A Guide to the New Essentialism. Chesham: Acumen.

_ (2005a). Physical Realism. Ratio (new series), XVIII (4), 371-84.

_ (2005b). Universals, the Essential Problem and Categorical Properties. Ratio (new series), XVIII (4), 462-72.

- (2008a). Categorical Dimensions and Causal Powers.Unpublished manuscript.

— (2008b). Essentialism and Natural Kinds. In S. Psillos \& M. Curd (Eds), The Routledge Companion to the Philosophy of Science (pp. 139-48): Routledge.

_ (forthcoming). The Categorical Dimensions of the Causal Powers. In Issues in the Metaphysics of Scientific Realism.

Ellis, B.D. \& Lierse, C. (1994). Dispositional Essentialism. Australasian Journal of Philosophy, 72 (1), 27-45. 
Esfeld, M. (Ed.) (2006). John Heil: Symposium on his Ontological Point of View. Frankfurt/Paris/Ebikon/Lancaster/New Brunswick: Ontos Verlag.

Fara, M. (2005). Dispositions and Habituals. Noûs, 39 (1), 43-82.

— (2006). Dispositions. Stanford Encyclopedia of Philosophy, 2008. Retrieved, 26

July. Online at: http://plato.stanford.edu/entries/dispositions/\#2.2

Feynman, R.P., Leighton, R.B. \& Sands, M. (1963). The Feynman Lectures on Physics. Reading, Mass.: Addison-Wesley.

Forrest, P. (2006). The Identity of Indiscernibles. In E.N. Zalta (Ed.), The Stanford Encyclopedia. Stanford: Stanford University.

Foster, J. (1982). The Case for Idealism. London: Routledge \& Kegan Paul.

Francescotti, R.M. (1999). How to Define Intrinsic Properties. Noûs, 33, 590-609.

Gibson, J.J. (1979). The Ecological Approach to Visual Perception. Boston: Houghthon Mifflin.

Gilbert, W. (1600). De Magnete (About the Magnet). Translated 1893 from Latin to English by Paul Fleury Mottelay. Dover Books.

Goldstein, J. (1999). Emergence as a Construct: History and Issues. Emergence: Complexity and Organization, 1 (1), 49-72.

Goodman, N. (1973). Fact, Fiction, and Forecast. 3rd ed. Indianapolis: BobbsMerrill.

Grajewski, M. J. (1944). The Formal Distinction of Duns Scotus. Washington, DC: The Catholic University of America Press.

Greene, R. (1712). The Principles of Natural Philosophy, In Which is Shewn the Insufficiency of the Present Systems, To Give Us Any Just Account of that Science: and the Necessity There is of Some New Principles, In order to Furnish us with a True and Real Knoweldge of Nature. Cambridge: Cambridge University Press.

Gribbin, J. (1998). The Search for Superstrings, Symmetry, and the Theory of Everything. Boston, New York, London: Little, Brown and Co.

- (2007). In Search of Superstrings, Symmetry, and the Theory of Everything. Boston, New York, London: Little, Brown and Co.

Gundersen, L. (2002). In Defence of the Conditional Account of Dispositions. Synthese, 130 (3), 389-411. 
Harari, H. (1979). A Schematic Model of Quarks and Leptons. Physics Letters B, 86 (1), 83-86.

Harré, R. (1970). The Principles of Scientific Thinking. London \& Basingstoke: Macmillan.

— (1997). Is There a Basic Ontology for the Physical Sciences? Dialectica, 51 (1), 17-34.

- (2001). Active Powers and Powerful Actors. Philosophy, 48 (Supp), 91-109.

- (2004). An Interpretation of the Philosophy of Niels Bohr, in the Light of the Philosophies of Kant and Husserl. Nordic Journal of Philosophy, 5 (2), 5-13.

Harré, R. \& Madden, E.H. (1973). Natural Powers and Powerful Natures. Philosophy, 48, 209-30.

— (1975). Causal Powers: A Theory of Natural Necessity. Totowa, New Jersey: Rowman and Littlefield.

_ (1976). Discussion: Causal Powers. Hume Studies, 2 (2), 95-103.

Hartle, J.B. (2003). Gravity: An Introduction to Einstein's General Relativity. San Francisco, CA: Addison Wesley.

Heil, J. (2003a). From an Ontological Point of View. Oxford: Clarendon Press.

— (2003b). Levels of Reality. Ratio (new series), XVI (3 September), 205-21.

— (2005a). Dispositions. Synthese, 144, 343-56.

— (2005b). Kinds and Essences. Ratio (new series), XVIII (4), 405-19.

— (2005c). Real Tables. The Monist, 88 (4), 493-509.

— (2006). Kinds and Essences. In A. Drewery (Ed.), Metaphysics (pp. 33-46). Malden, MA; Oxford; Victoria, Australia: Blackwell Publishing.

— (2007). 'Reply to Sharon Ford'. In G. Romano (Ed.), Symposium: From an Ontological Point of View, Vol. 6, (pp. 82-85). (Reply to: Ford, S.R. (2007), 'An Analysis of Properties in John Heil's "From an Ontological Point of View"', SWIF Philosophy of Mind Review, G. Romano (ed.), 6 (2), pp. 42-51.) Philosophy of Mind Review: SWIF. http://gxserver.uniba.it/mind/swifpmr/ 0620072.pdf

Hochberg, H. (1999a). Complexes and Consciousness Vol. 26. Stockholm: Thales. — (1999b). D.M. Armstrong, A World of States of Affairs. Noüs, 33 (3), 473-95. 
- (2001). The Positivist and the Ontologist: Bergmann, Carnap and Logical Realism. Amsterdam - Atlanta, GA: Rodopi B. V.

Hoffman, J. \& Rosenkranz, G.S. (1994). Substance Among Other Categories. Cambridge: Cambridge University Press.

— (1997). Substance: Its Nature and Existence. London: Routledge.

Holbo, J. Retrieved 10 January, 2007, Online at http://crookedtimber.org/2006/09/13/ more-nietzsche-on-kant-thanks-ill-be-here-all-week/

Holton, R. (1999). Dispositions All the Way Round. Analysis, 59 (1), 9-14.

Hume, D. (1739). A Treatise of Human Nature. Oxford: Clarendon Press.

— (1993 [1740, 1777 post-humous edition]). An Enquiry Concerning Human Understanding. In E. Steinberg. (Ed.), An Enquiry Concerning Human Understanding; [with] A Letter from a Gentleman to His Friend in Edinburgh; [and] An Abstract of a Treatise of Human Nature (2nd ed.). Publisher Indianapolis: Hackett Pub. Co.

- (Ed.) (1962). A Treatise of Human Nature: Book 1, Of the Understanding. Glasgow: William Collins Sons.

Icke, V. (1995). The Force of Symmetry. Cambridge: Cambridge University Press.

Johnston, M. (1992). How to Speak of the Colors. Philosophical Studies, 68, 221-63.

Kaluza, T. (1921). Zum Unitätsproblem der Physik. Sitzungsberichte Preussische Akademie der Wissenschaften, 54, 966-72.

Kant, I. (1786/1909). The Metaphysical Foundations of Natural Science. In Kant's Prolegomena and Metaphysical Foundations of Natural Science (pp. 137-245). London: George Bell and Sons.

Kim, J. (1973). Causation, Nomic Subsumption, and the Concept of Event. The Journal of Philosophy, 70 (8), 217-36.

- (1990). Supervenience as a Philosophical Concept. Metaphilosophy, 21 (1-2), 127.

Klein, O. (1926). Quantentheorie und Fünfdimensionale Relativitätstheorie. Zeitschrift für Physik A Hadrons and Nuclei, 37 (12), 895-906.

Krauss, L.M. (2005). Hiding in the Mirror: The Mysterious Allure of Extra Dimensions, From Plato to String Theory and Beyond. New York: Viking; Penguin Group. 
Kribs, D.W. \& Markopoulou, F. (2005). Geometry from Quantum Particles, 2009. Retrieved, 11 Oct 2005. Online at: http://arxiv.org/abs/gr-qc/0510052

Lange, M. (2002). An Introduction to the Philosophy of Physics: Locality, Fields, Energy, and Mass. Oxford: Blackwell Publishers.

- (2004). A Note on Scientific Essentialism, Laws of Nature, and Counterfactual Conditionals. Australasian Journal of Philosophy, 82 (2), 227-41.

Langton, R. \& Lewis, D. (1998). Defining 'Intrinsic'. Philosphy and Phenomenological Research, 58, 333-45.

Larmor, J. (1919). On Generalized Relativity in Connection with Mr. W.J. Johnston's Symbolic Calculus. Proceedings of the Royal Society of London. Series A, Containing Papers of a Mathematical and Physical Character, 96 (678), 33463.

Leibniz, G.W. (1969). Philosophical Papers and Letters. 2nd ed. Dordrecht, Holland: Reidel.

- (1996). New Essays on Human Understanding. Cambridge; New York: Cambridge University Press.

— ([1686] 1973). Discourse on Metaphysics. In G.H.R. Parkinson (Ed.), Leibniz: Philosophical Writings. London, Melbourne \& Toronto: Dent.

Levi, I. (2003). Dispositions and Conditionals. In H. Lillehammer, G. Rodríguez Pereyra \& D.H. Mellor (Eds), Real Metaphysics: Essays in Honour of D.H. Mellor (pp. 137-53). London; New York: Routledge.

Lewis, D. (1983a). Extrinsic Properties. Philosphical Studies, 44, 197-200.

— (1983b). New Work for a Theory of Universals. Australasian Journal of Philosophy, 61 (December), 343-77.

- (1986a). Against Haecceity. In On the Plurality of Worlds (pp. 220-47). Oxford: Blackwell.

— (1986b). Against Structural Universals. Australasian Journal of Philosophy, 64 (March), 25-46.

— (1986c). On the Plurality of Worlds. Oxford: Blackwell.

—_ (1986d). Philosophical Papers Vol. II. Oxford: Oxford University Press.

— (1992). Critical Notice of D M Armstrong, "A Combinatorial Theory of Possibility". Australasian Journal of Philosophy, 70 (2), 211-24. 
_ (1993). Many, but Almost One. In J. Bacon, K. Campbell \& L. Reinhardt (Eds), Ontology, Causality and Mind: Essays in Honour of D M Armstrong (pp. 2338). New York: Cambridge University Press.

— (1999a). Papers in Metaphysics and Epistemology. Cambridge: Cambridge University Press.

— (1999b). Papers in Metaphysics and Epistemology. Philosophical Review, 110 (1), 77-79.

- (2000). Causation as Influence. The Journal of Philosophy, 97 (4), 182-97.

— (2001). Forget About the 'Correspondence Theory of Truth'. Analysis, 61 (4), 275-80.

Locke, J. (1924). An Essay Concerning Human Understanding. Oxford: Clarendon Press.

Mackie, P. (2000). Review of Hoffman and Rosenkranz (1994) Mind, 109, 149-52.

MacKinnon, E. (1975). A Reinterpretaiton of Harre's Copernican Revolution. Philosophy of Science, 42 (1), 67-69.

Madden, E.H. (1972). Discussion: R. Harre's The Principles of Scientific Thinking. Southern Journal of Philosophy, 10 (Spring), 23-32.

_ (1973). Scientific Explanations. Review of Metaphysics, 26 (4), 723-43.

Madden, E.H. \& Hare, P. (1971). The Powers that Be. Dialogue, 10, 12-30.

Madden, E.H. \& Harré, R. (1973). In Defence of Natural Agents. The Philosophical Quarterly, 23 (91), 117-32.

Madden, E.H. \& Sachs, M. (1972). Parmenidean Particulars and Vanishing Elements. Studies in History and Philosophy of Science, 3, 151-66.

Malzkorn, W. (2000). Realism, Functionalism and the Conditional Analysis of Dispositions. Philosophical Quarterly, 50 (201), 452-69.

Martin, C.B. (1993). Power for Realists. In J. Bacon, K. Campbell \& L. Reinhardt (Eds), Ontology, Causality and Mind: Essays in Honour of D M Armstrong (pp. 175-85). New York: Cambridge University Press.

— (1994). Dispositions and Conditionals. The Philosophical Quarterly, 44 (174), $1-8$. 
_ (1996a). Final Replies to Place and Armstrong. In D.M. Armstrong, U.T. Place, C.B. Martin \& T. Crane (Eds), Dispositions: A Debate (pp. 163-92). New York: Routledge.

— (1996b). Properties and dispositions. In D.M. Armstrong, U.T. Place, C.B. Martin \& T. Crane (Eds), Dispositions: A Debate (pp. 71-87). New York: Routledge.

— (1996c). Replies to Armstrong and Place. In D.M. Armstrong, U.T. Place, C.B. Martin \& T. Crane (Eds), Dispositions: A Debate (pp. 126-46). New York: Routledge.

— (1997). On the Need for Properties: The Road to Pythagoreanism and Back. Synthese, 112 (2), 193-231.

Martin, C.B. \& Heil, J. (1998). Rules and Powers, Philosophical Perspectives (Vol. 32, pp. 283-312).

— (1999). The Ontological Turn. Midwest Studies in Philosophy, XXIII, 34-60.

Mellor, D.H. (1974). In Defense of Dispositions. Philosophical Review, 83 (2), 157 81.

Molnar, G. (1999). Are Dispositions Reducible? Philosophical Quarterly, 49 (194), 117.

— (2003). Powers: A Study in Metaphysics. New York: Oxford University Press.

Mumford, S. (1994). Dispositions, Supervenience and Reduction. Philosophical Quarterly, 44 (177), 419-38.

— (1998). Dispositions. New York: Oxford University Press.

- (2004). Laws in Nature. London: Routledge.

Nerlich, G. (1976). The Shape of Space. Cambridge New York: Cambridge Univesity Press.

— (1993a). The Shape of Space. Second ed. Cambridge: Cambridge University Press.

- (1993b). What Spacetime Explains: Metaphysical Essays on Space and Time. Cambridge: Cambridge University Press.

Nietzsche, F. W. (1886/1923). Beyond Good and Evil: Prelude to a Philosophy of the Future. 4th ed. Edinburgh: T.N. Foulis. 
Pati, J.C. \& Salam, A. (1974). Lepton Number as the Fourth "Color". Physical Review D., $10(1), 275-89$.

Place, U.T. (1996a). Conceptualism and the Ontological Independence of Cause and Effect. In D.M. Armstrong, U.T. Place, C.B. Martin \& T. Crane (Eds), Dispositions: A Debate (pp. 153-62). New York: Routledge.

(1996b). A Conceptualist Ontology. In D.M. Armstrong, U.T. Place, C.B. Martin \& T. Crane (Eds), Dispositions: A Debate (pp. 49-67). New York: Routledge.

— (1996c). Dispositions as Intentional States. In D.M. Armstrong, U.T. Place, C.B. Martin \& T. Crane (Eds), Dispositions: A Debate (pp. 19-32). New York: Routledge.

— (1996d). Intentionality as the Mark of the Dispositional. Dialectica, 50 (2), 91120.

— (1996e). Structural Properties: Categorical, Dispositional or Both? In D.M. Armstrong, U.T. Place, C.B. Martin \& T. Crane (Eds), Dispositions: A Debate (pp. 105-25). New York: Routledge.

_ (1999a). Intentionality and the Physical: A Reply to Mumford. The Philosophical Quarterly, 49 (195), 225-31.

— (1999b). Vagueness as a Mark of Dispositional Intentionality. Acta Analytica, 14 (23), 91-109.

Priestley, J. (1782). Disquisitions Relating to Matter and Spirit, Vol 1-2. Birmingham, England: Pearson and Rollason.

Prior, E. (1985). Dispositions. Aberdeen: Aberdeen University Press ; Atlantic Highlands N.J. : Humanities Press.

Prior, E.W., Pargetter, R. \& Jackson, F. (1982). Three theses about dispositions. American Philosophical Quarterly, 19 (3), 251-58.

Quine, W.V.O. (1976). Whither Physical Objects? In R.S. Cohen, P.K. Feyerabend \& M.W. Wartofsky (Eds), Essays in the Memory of Imre Lakatos, Boston Studies in the Philosophy of Science (Vol. XXXIX). Dordrecht: D. Reidel.

Ravndal, F. (2003). Scalar Gravitation and Extra Dimensions. Paper presented at the Gunnar Nordstrom Symposium on Theoretical Physics, August 27-30 from http://arxiv.org/abs/gr-qc/0405030 
Redhead, M. (1988). A Philosopher Looks at Quantum Field Theory. In H.R. Brown \& R. Harré (Eds), Philosophical Foundations of Quantum Field Theory (pp. 923). Oxford: Clarendon Press.

Redhead, M.L.G. (1975). Symmetry in Intertheory Relation. Synthese, 32, 77-112.

— (1982). Quantum Field Theory for Philosophers. PSA 1982: Proceedings of the 1982 Biennial Meeting of the Philosophy of Science Association, 2, 57-99.

Reid, T. (1788/1819). Essays on the Powers of the Human Mind. Edinburgh: Bell and Bradfute.

Rickles, D. \& French, S. (2006). Quantum Gravity Meets Structuralism: Interweaving Relations in the Foundations of Physics. In D. Rickles, S. French \& J.T. Saatsi (Eds), The Structural Foundations of Quantum Gravity (pp. 1-35): Oxford University Press.

Rissler, J.D. (2006). Does Armstrong Need States of Affairs? Australasian Journal of Philosophy, 84 (2), 193-209.

Robb, D. (2005). Qualitative Unity and the Bundle Theory. The Monist, 88 (4), 46692.

Robinson, H. (1982). Matter and Sense: A Critique of Contemporary Materialism. Cambridge: Cambridge University Press.

Robinson, H.M. (2004). Substance. Stanford Encyclopedia of Philosophy, Retrieved, 18 August, 2008. Online at: http://plato.stanford.edu/entries/substance

Rosenberg, A. (1984). Mackie and Shoemaker on Dispositions and Properties. Midwest Studies in Philosophy, 9, 77-92.

Rovelli, C. (1997). Halfway Through the Woods: Contemporary Research on Space and Time. In J. Earman \& J.D. Norton (Eds), The Cosmos of Science: Essays of Exploration (pp. 180-223). Pittsburgh: University of Pittsburgh Press.

Russell, B. (1911). The Problems of Philosophy. London; New York; Toronto: Oxford University Press.

— (1925). The ABC of Relativity. London: Kegan Paul.

— (1954 [1927]). The Analysis of Matter. London: George Allen and Unwin.

— (1959 [1927]). The Analysis of Matter 2nd ed. London: George Allen and Unwin. 
— (1970 [1927]). The Structure of the Atom. In An Outline of Philosophy (pp. 10312, Chapter IX). London: Allen and Unwin.

(1985). My Philosophical Development. London: Unwin Paperbacks.

Ryle, G. (1949). The Concept of Mind. New York: Barnes \& Noble.

Scotus, D. (1994). Ordinatio II. In P.V. Spade (Ed.), Five Texts on the Mediaeval Problem of Universals: Porphyry, Boethius, Abelard, Duns Scotus, Ockham. Indianapolis: Hackett.

_ (1997). Reportata Parisiensia. In R.N. Bosley \& M.M. Tweedale (Eds), Basic Issues in Medieval Philosophy. Peterborough, Ontario: Broadview Press.

Shoemaker, S. (1980a). Causality and Properties. In P. Van Inwagen (Ed.), Time and Cause (pp. 109-35). Dordrecht, Netherlands: D. Reidel Publishing.

— (1980b). Properties, Causation, and Projectibility. In L.J. Cohen \& M. Hesse (Eds), Applications of Inductive Logic (pp. 291-312). Oxford: Clarendon Press.

- (1984a). Causality and Properties. In Identity, Cause, and Mind: Philosophical Essays (pp. 206-33). Cambridge: Cambridge University Press.

— (1984b). Identity, Properties, and Causality. In Identity, Cause, and Mind: Philosophical Essays (pp. 234-60). Cambridge: Cambridge University Press.

- (1998). Causal and Metaphysical Necessity. Pacific Philosophical Quarterly, 79, 59-77.

— (2003). Identity, Cause, and Mind. 2nd ed. Oxford; New York: Oxford University Press.

Shoemaker, S., Swinburne, R., Mackie, J.L., Mellor, H., Hesse, M.H. \& UllmannMargalit, E. (1980). Comments and Replies: The Shoemaker-Swinburne Session. In L.J. Cohen \& M. Hesse (Eds), Applications of Inductive Logic (pp. 321-32). Oxford: Clarendon Press.

Shope, R.K. (1988). Powers, Causation, and Modality. Erkenntnis, 28, 321-62.

Simons, P. (2005). Negatives, Numbers, and Necessity Some Worries About Armstrong's Version of Truthmaking. Australasian Journal of Philosophy, 83 (2), 253-61.

Smith, A. D. (1977). Dispositional Properties. Mind, 86 (July), 439-45. 
Smolin, L. (1991). Space and Time in the Quantum Universe. In A. Ashtekar \& J. Stachel (Eds), Conceptual Problems of Quantum Gravity. Boston: Birkhauser.

— (1997). The Future of Spin Networks. Retrieved, 17 Feb 1997. Online at: http://arxiv.org/abs/gr-qc/9702030

— (2000). Three Roads to Quantum Gravity. London: Phoenix.

- (2006). The Trouble with Physics: The Rise of String Theory, the Fall of a Science and What Comes Next. Penguin Books.

Sparber, G. (2006). Powerful Causation. In M. Esfeld (Ed.), JohnHeil Symposium on His Ontological Point of View. Frankfurt, Main: Ontos-Verlage.

Spinoza (1632-1677). Ethics. Translated by A. Boyle. Heron Books.

Stachel, J. (1998a). Introduction. In J. Stachel (Ed.), Einstein's Miraculous Year (pp. 3-27). Princeton, N.J.: Princeton University Press.

— (Ed.) (1998b). Einstein's Miraculous Year. Princeton, N.J.: Princeton University Press.

Swinburne, R. (1980a). Comments and Replies: The Shoemaker-Swinburne Session. In L.J. Cohen \& M. Hesse (Eds), Applications of Inductive Logic (pp. 321-32). Oxford: Clarendon Press.

- (1980b). Properties, Causation, and Projectibility: Reply to Shoemaker. In L.J. Cohen \& M. Hesse (Eds), Applications of Inductive Logic (pp. 313-20). Oxford: Clarendon Press.

Teller, P. (1982). Comments on the Papers of Cushing and Redhead: "Models, HighEnergy Theoretical Physics and Realism" and "Quantum Field Theory for Philosophers", PSA: Proceedings of the Biennial Meeting of the Philosophy of Science Association: Symposia and Invited Papers (Vol. 2).

— (1995). An Interpretative Introduction to Quantum Field Theory. Princeton, New Jersey: Princeton University Press.

Tooley, M. (1977). The Nature of Laws. Canadian Journal of Philosophy, 7, 667-98. van Dongen, J. (2002). Einstein and the Kaluza-Klein Particle. Studies in History and Philosophy of Modern Physics, 33, 185-210.

Weatherson, B. (2001). Intrinsic Properties and Combinatorial Principles. Philosphy and Phenomenological Research, 63, 365-80. 
Weingard, R. (1991). The Vacuum and Unification. In S. Saunders \& H.R. Brown (Eds), The Philosophy of Vacuum (pp. 197-215). Oxford: Clarendon Press.

Wheeler, J. A. (1962). Geometrodynamics. New York: Academic Press.

Wuensch, D. (2003). The Fifth Dimension: Theodor Kaluza's Ground-Breaking Idea. Annalen der Physik, 12 (9), 519-42. 


\section{INDEX}

aether 4, 212

affordance ... 191

Aquinas $199,200,202,203,260$

Armstrong, David .. iii, iv, v, 1, 2, 1-55, 8, 9, 11, 13, $15,16,17,18,19,20,21,22,23,24,25,29,30$, $31,32,33,34,35,36,37,38,39,40,41,42,43$, $44,46,47,48,49,52,53,54,80,87,94,95,118$, $119,120,121,122,126,127,129,131,132,134$, $137,138,139,141,148,150,154,160,161,162$, $173,176,179,197,204,208,211,217,233,248$, $253,256,261,262,263,264,265,267,269,270$, 272,273

Categoricalism.....9, 10-55, 57, 94, 99, 107, 149, $160,161,180$

Central State Materialism

Central-State Materialism..

Combinatorialism ....................................22, 261

Contingent Identity Thesis................... 17, 22, 54

Intersection Theory ............................. 50, 51, 54

atomism ...................................... 4, 168, 173, 212

Barber Pole Effect......................................viii, 229

Baxter

Aspect Theory …........................................ 9, 50

Baxter, Donald ............................... 49, 50, 211, 262

Bergmann, Gustav .....................................21, 267

Bigelow, John ............................................20, 20,

Bilson-Thompson, Sundance O ..... 7, 164, 186, 243

Bilson-Thompson, Sundance O. ................... 7, 186

Bird, Alexander.....v, 1, 7, 11, 31, 39, 43, 44, 47, 52, $53,54,57,70,90,102,122,211,232,241,242$, $245,253,262,263,264$

Black, Robert $44,47,94,102,239,263$

Blackburn, Simon 170,206

Boscovich, Roger... vii, 10, 164, 165, 167, 170, 183, 184, 185, 187, 188, 192, 194, 263

Boyd, Richard ................................................... 61

Brown, H........................................................ 171

Bundle Theory ....................................... 49, 201, 217

Campbell, Keith ... vii, 10, 73, 76, 77, 78, 79, 80, 84, $164,165,167,168,169,197,263,269$

Categoricalismiv, v, 9, 13, 15, 32, 36, 37, 39, 43, 44, $53,55,57,94,99,107,149,157,160,161,163$, $176,211,255$

causal interaction ................ 101, 108, 176, 207, 242

causal potentialities..8, 61, 62, 64, 65, 67, 87, 92, 93 causal process .....101, 104, 108, 109, 111, 112, 117, $119,121,123,128$

causation.12, 13, 24, 53, 54, 59, 134, 149, 163, 173, $176,177,178,180,191,212,253$

singular ..................................... 35, 37, 38, 55 strong....13, 53, 54, 149, 163, 176, 178, 191, 212

Choi, Sungho …...................................... 11, 264

circumstances....1, 11, 16, 19, 20, 57, 61, 62, 65, 67, $82,83,86,88,89,90,91,92,96,104,105,106$, $109,111,112,120,121,123,124,126,127,129$, $138,139,141,157,159,183,192,203,254$ contingent .........67, 106, 120, 121, 127, 159, 203

Conceptualism .................................See Place, Ullin
Connectibility Argument ...........See Martin, Charles Copenhagen View ........................................... 185 Crane, Tim ... 137, 211, 238, 261, 262, 264, 270, 271 de Spinoza, Benedict.................... 10, 165, 168, 274 dimensional asymmetry ............................ 248, 250 dimensions 7, 82, 104, 105, 106, 109, 111, 112, 113, $114,115,117,118,120,121,123,127,128,158$, $199,212,221,224,225,228,229,230,231,232$, 241, 244, 246, 247, 249, 251, 255, 258, 259

Dipert, Randall. $147,207,264$

Dispositional Essentialism

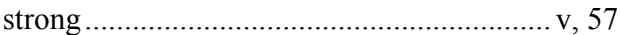
weak …………………………….......... 57, 71

dispositions

irreducible......39, 40, 44, 52, 53, 54, 55, 99, 149, $160,161,162,176,177,253,255$

mental ............................................. 16, 17 supervenient.............................................20, 22 unmanifested................................... 36, 37, 158

Dodd, James............................... 211, 228, 238, 264

Drewery, Alice

Dynamism.................................. 164, 166, 169, 171

Earman, John ................................... 11, 264, 272

Einstein, Albert 5, 6, 7, 224, 225, 264, 265, 267, 274 relativity

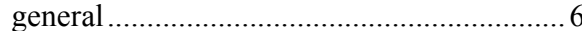
special.................................... 6, 49, 74, 166

Ellis, Brian .... iii, iv, vi, 1, 2, 4, 6, 7, 8, 9, 11, 44, 57, $69,71,82,101,102,103,104,105,106,107$, $108,109,111,112,113,114,115,116,117,118$, $119,120,121,122,123,125,126,127,128,132$, $158,160,199,211,242,246,97-128,262,265$ Hierarchy of Natural Kinds... 101, 102, 107, 108, 109

New Essentialism . iv, vi, 71, 101, 104, 107, 111, 128, 129, 197, 199, 255, 262, 265

New Essentialism ........................................... 71

New Essentialism ..................................... 97-128

energy transfer process ............................. 101, 108

Fara, Michael ....................................................... 11

Faraday, Michael ......................................... 5, 165

fermions ......221, 225, 226, 227, 228, 238, 240, 241, $243,246,247,249,250,260$

Feynman, Richard .............................. 82, 235, 266 field

electromagnetic..................... 125, 226, 227, 238

Field Theory... iv, vii, viii, $6,80,168,171,178,186$, $189,197,216,222,234,257,272,274$

forces ..... 7, 72, 74, 83, 84, 108, 119, 166, 171, 172, $177,188,189,192,194,209,222,223,226,227$, $228,232,234,237,238,241,249,258,260$

Foster, John....................... $8,72,73,74,75,76,266$ Foundational Monism .......... 97, 164, 177, 216, 254 Foundationism ................. vi, 76, 77, 78, 80, 84, 169 gauge bosons.......222, 223, 224, 225, 226, 227, 228, $229,230,231,232,233,234,238,239,240,244$, $247,248,249,250,258,259$ 
generative mechanism.126, 172, 178, 180, 181, 182, 195,257

Geometrodynamism.................................. 77, 169

Gilbert, W.................................................... 165

Goodman, Nelson ............................................ 266

Great Field ......10, 164, 186-96, 188, 189, 190, 191, 192, 194, 195, 196, 211, 214, 216, 218, 219, 220, 221, 250, 251, 257, 258, see also; Harré, Rom \& Madden, E.H

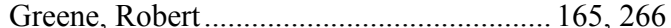

Gribbin, John ...7, 224, 226, 227, 237, 238, 264, 266

grue 11,266

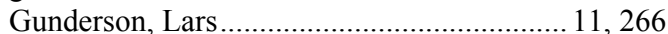

haecceity ........ 7, 24, 47, 63, 221, 234, 235, 239, 245

Harré, Rom ... iv, vii, 10, 12, 126, 164, 163-96, 165, $169,171,172,173,174,175,176,177,178,179$, $180,181,182,183,184,185,186,187,188,189$, 190, 191, 193, 194, 195, 196, 202, 206, 207, 211, $214,216,218,219,220,221,222,238,239,240$, $244,245,246,250,251,257,258,259,266,269$, 272

Heil, John ....iv, vi, 1, 4, 9, 11, 70, 71, 116, 143, 144, $145,147,148,149,150,151,153,154,155,156$, $157,158,159,160,161,162,163,203,207,208$, 209, 217, 246, 256, 265, 267, 270, See also, Identity Theory of Properties Identity Theory of Properties...... iv, vi, 116, 143, $151,153,156,158,159,160,162,210,246,256$

Heisenberg, Werner 5, 237, 248

Hesse, Mary .............................................. 273, 274

Hochberg, Herbert........................... 39, 54, 211, 253

Holton, Richard.................................. 147, 207, 267

Hume

distinctness ............................................. 173, 178

Principle of Independence ..... 173, 177, 181, 260 Regularity Thesis of Causality.............. 175, 191

Hume, David. 86, 132, 135, 150, 172, 173, 174, 175, 176, 177, 178, 180, 186, 191, 206, 267

Icke, Vincent............................. 226, 227, 228, 268

identity

of particulars ...................................................... 47

of properties......................... 54, 76, 91, 94, 96

token ……................................................. 36

type ................................. 16, 17, 18, 22, 36

image

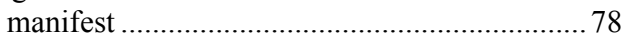

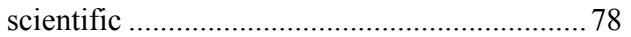

Individual

Aristotelian ....182, 183, 194, 195, 222, 238, 239, 250

Parmenidean ... vii, 172, 180, 182, 183, 185, 186, 187, 188, 189, 190, 194, 195, 207, 211, 215, 216, $221,222,226,238,239,240,250,257,258,259$, 269

Strawsonian

individuals

bundle theory ...........29, 190, 202, 203, 215, 219

transcendental (TI).

$63,233,234$

individuation

of objects and properties..... 25, 63, 79, 163, 187 , 189, 203, 209, 212, 215, 216, 219, 221, 233, 234, 250 instantiation. $1,15,25,30,31,32,34,35,39,40,48$, $49,50,51,53,54,61,62,64,102,160,170,201$, 217

contingent $48,49,54$ necessary 51

Jackson, Frank $11,18,149,271$

Kaluza-Klein................164, 224, 225, 228, 268, 274

Kant, Immanuel ....10, 164, 165, 166, 167, 172, 180, $183,184,187,188,192,194,207,266,268$

Kim, Jaegwon ............................................ 63, 268

Krauss, Lawrence M................................... 237, 268

Lange, Marc................................. 11, 169, 197, 268

Law of Continuity......... vii, 183, 184, 185, 186, 196 laws

causal ..............64, 107, 118, 121, 123, 213, 214 contingent ........................................2 21, 37, 44 higher-order ........................ 34, 35, 39, 42, 253 necessary .....19, 20, 53, 54, 64, 85, 95, 121, 126, $127,160,203,204,255$ strong $44,54,55,57$

Leibnitz, G.W. ................................................. 165

Leibniz ............................... 10, 165, 202, 268, 269

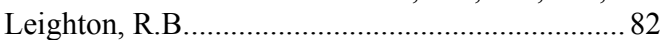

Levi, Isaac................................................. 11, 269

Lewis, David............................................................ 30

Light-like Network Account ... iv, 10, 164, 221, 222, $223,225,226,228,230,231,233,241,245,258$, 259,260

Locke, John................143, 144, 149, 155, 165, 269

Loop Quantum Gravity ............ 7, 79, 164, 186, 243

Mackie, J.L. ............................. 218, 269, 272, 273

Madden, E.H.....vii, 10, 164, 163-96, 171, 173, 174, $178,179,182,184,185,186,189,191,195,206$, $222,257,264,266,269$

Malzkorn, Wolfgang ..................................... 11, 269

Martin, Charles ..4, 11, 21, 44, 54, 70, 131, See also, Identity Theory of Properties

Connectibility Argument .................................. 40 Dual-aspect Theory...................................... 9, 143

Identity Theory of Properties...... iv, vi, 116, 143, $151,153,156,158,159,160,162,210,246,256$ Two-Aspect Theory.

Martin, Charlie ... iv, v, vi, 1, 4, 9, 11, 21, 39, 40, 44 $53,54,70,71,72,76,116,131,143,145,146$, $147,148,149,150,151,153,154,155,156,157$, $158,159,160,161,162,163,176,197,209,211$, $240,246,253,256,259,261,262,264,269,270$, 271

material point $5,6,165,167,170$

Maxwell, James Clerk................ 5, 6, 165, 225, 264

Meinongian objection .......................................... 158

Mellor, Hugh............................ 263, 269, 270, 273

Molnar, George vi, 11, 44, 61, 67, 71, 72, 73, 74, 75, $76,78,80,81,82,83,84,85,95,117,118,119$, $163,197,203,205,210,211,254,270$

multiple-realisability ............................... 17, 18, 36

Mumford, Stephen ......7, 11, 18, 122, 140, 141, 156, $211,241,255,256,262,270,271$

necessity

causal 63,171

metaphysical 63

Nerlich, Graham $\quad 10,169,246$ 
New Essentialismiv, vi, 71, 101, 104, 107, 111, 128, 129, 197, 199, 255, 262, 265

Nietzsche, Friedrich .................................. 73, 270

Nominalism........................................... 29, 199, 261

Norman, Robert ……......................................... 165

objects

complex85, 86, 87, 106, 146, 158, 160, 200, 205, 218

concrete .

$144,149,209$

constituent.

63

distinct $.57,67,77,78,83,84,85,87,96,97,99$, $123,124,125,164,177,193,201,202,209,212$, $216,221,249,250,254,257,260$

Pargetter, Robert .................... 11, 20, 149, 262, 271

particle theory .7, 9, 72, 83, 101, 108, 168, 185, 210, $222,226,233,234,235,236,237,238,247,248$ particulars

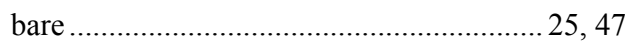

thick .................................................. 29, 49, 50

thin ............................... 25, 29, 47, 49, 51, 176

Pauli ........................................................... 241, 250

Pauli Exclusion Principle ........................... 241, 250

perception

direct........................................ 29, 69, 70, 96, 117

Phenomenalism.............................. See Ryle, Gilbert

Place

Conceptualism iv, vi, 9, 131, 137, 140, 149, 177, 199, 205, 256, 271

Place, Ulliniv, vi, 1, 4, 9, 11, 44, 131, 132, 133, 134, $137,128-41,137,138,139,140,141,145,149$, 177, 199, 256, 261, 262, 264, 270, 271

Conceptualism

$128-41,149$

Planck's constant ........................................... 78, 79

point-moments .......24, 188, 213, 244, 247, 249, 259

possibility.......iv, $4,22,41,52,78,92,93,106,120$, $132,154,158,166,174,181,198,199,203,204$, $205,213,220,228,231,244,245,247,250,257$, 258,260

possible worlds ..........18, 20, 32, 45, 46, 47, 61, 218 power-net. 159 powers

causal.....10, 18, 59, 64, 67, 68, 73, 95, 102, 103, $104,105,106,107,108,109,111,112,113,114$, $115,117,118,120,121,122,123,124,125,126$, $127,128,171,173,199,255$

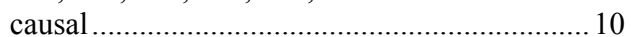

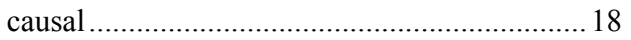

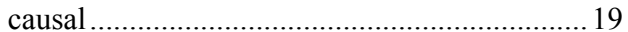

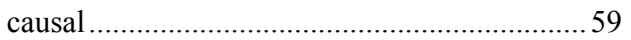

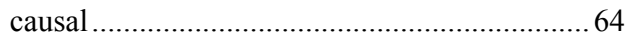

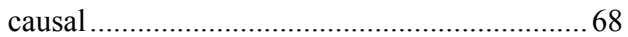

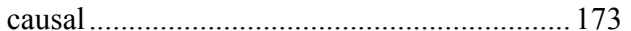

conditional $\ldots 8,61,62,65,67,85,86,91,93,94$, 95

conditional

$61-76$

Priestley, Joseph.................................................. 165

Principle of Independence..... vi, 86, 87, 92, 96, 124, $132,173,176,177,254,256$

Prior, Elisabeth.........11, 18, 149, 211, 244, 261, 271 properties

extrinsic ................................................. 76,84 genuine $. .8,61,62,64,65,67,75,76,80,81,84$, $92,96,163,254$ higher-order ........25, 65, 105, 150, 218, 220, 233 historical ................................................. 59, 64 identity condition ............................................. 207 intrinsic...................91, 101, 103, 201, 206, 254 mere-Cambridge ................ 60, 64, 65, 75, 83, 89 non-relational.................................... 25, 49, 143 particularising ................................................ 26 power-qualities ......116, 143, 144, 150, 151, 153, $154,156,159,160,161,162,210,246$ relational..............25, 49, $62,64,75,83,88,143$ S-properties.

S-Properties ............. vi, 80, 82, 85, 95, 163, 254

Property-monism ......................................... 156 qualia ............................ 70, 71, 72, 145, 148, 240 quanta......................................... 5, 79, 234, 237 quantum mechanics....... $5,6,79,108,205,210,223$, $225,235,243$

quiddity. $44,61,62,67,68,76,94,95,97,102,109$, $111,112,113,114,115,116,118,127,163,177$, $211,221,233,239,241,245,254,255,257$

Redhead, Michael ......7, 63, 186, 234, 235, 272, 274 reduction ...11, 12, 16, 17, 18, 19, 20, 21, 22, 36, 37, $62,92,95,96,133,204,211,235,254$

metaphysical $\mathrm{v}, 17$ regress

Bradley's instantiation............... 48, 54, 201, 217

Harré's explanatory ......................................... 181

Swinburne.............................................62, 120

Reid, Thomas ............................................ 165, 272

relations

external ............... 51, 62, 177

extrinsic ..... 75, 77, 78, 83, 85, 86, 87, 90, 92, 96, 177,254

mere-Cambrdige ............................................. 59

mere-Cambridge ....59, 60, 62, 63, 64, 65, 75, 82, $83,87,89,177$

necessitating ...32, 33, 34, 36, 39, 41, 42, 43, 179, 253

spatiotemporal ...6, 67, 75, 76, 80, 82, 83, 84, 85, $91,96,103,104,106,115,120,122,133,163$, $177,254,255$

Rissler, James $211,262,272$

Robinson, Howard215, 216, 217, 219, 233, 249, 272

Rosenberg, Alexander..........................................6 69

Rovelli, Carlo... 7, 232, 236, 238, 239, 242, 243, 272

Russell, Bertrand....4, 5, 30, 212, 213, 214, 223, 272

Ryle, Gilbert ................................... 15-17, 37, 132

Phenomenalism................................................ 15

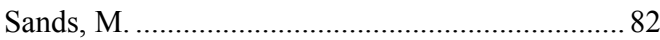

Schrödinger, Erwin ................................................ 5

Shoemaker

Causal Theory of Property. iv, v, 8, 9, 10, 57, 59, $62,63,65,67,69,70,71,72,75,78,80,84,85$, $92,95,96,97,99,119,163,177,211,249,253$, $254,255,262$

Shoemaker, Sydney .iv, v, 4, 8, 9, 10, 11, 44, 57, 59, $61,62,63,64,65,67,69,70,71,75,76,80,81$, $82,83,84,85,86,87,89,90,91,92,93,94,95$, $96,99,118,132,147,154,162,163,177,183$, $186,201,211,249,253,254,262,272,273,274$ Causal Theory of Properties ........................ 9, 10 Simons, Peter $50,211,262,273$ 
singularities...38, 125, 171, 186, 187, 188, 189, 190, $192,194,195,207,211,214,222,236,257,258$, 259

Smolin, Lee. $225,232,243,262,273$

space-fillers ... 223

space-occupants 73,75

spacetime $6,7,8,10,77,78,79,107,122,123,164$, $165,168,169,189,190,193,195,197,198,207$, $212,213,214,218,220,221,222,223,224,225$, $228,230,232,236,237,240,241,242,244,245$, $246,248,249,250,257,258,259,260$

physicalised ................................... 77, 164, 169 spacetime curvature ..7, 77, 168, 169, 207, 218, 221, 224, 232, 237, 244, 246

Stachel, John ........................ 264, 265, 273, 274 state of affairs..21, 22, 23, 24, 29, 31, 32, 33, 34, 35, $37,39,41,47,48,49,50,51,54,131,132,133$, $134,137,138,139,140,145,146,172,191$ atomic 23,48 higher-order ..................................... 22, 23, 33 molecular ........................................................ 23 state of affairs types .....23, 24, 31, 33, 34, 35, 37, 41 structure

categorical.

114,140 powerful $4,128,189,251,256$

substance iv, 2, 4, 5, 20, 74, 101, 106, 134, 149, 168, $172,188,190,191,193,194,195,197,206,209$, $212,213,214,215,216,217,219,220,221,234$, $248,249,260,272$
Substance-monism $67,85,188,190,191$ Swinburne, Richardiv, v, 4, 8, 10, 62, 65, 67, 68, 69, $70,71,72,75,76,79,94,95,117,118,120,128$, $149,197,198,200,206,207,209,240,253,259$, 273,274 regress... 120,149

Tooley, Michael ..........................................31, 274 truthmaker.16, 21, 22, 42, 44, 55, 95, 132, 133, 137 , $138,139,141,154,155,156,203,256$

non-categorical . 138

Two-Aspect Theory ...................See Martin, Charles

Ullmann-Margalit, Edna ....................................... 273

Universalism .............................................29, 199

universals .. 18, 22, 23, 24, 25, 30, 31, 32, 33, 34, 35, $36,38,40,41,42,43,45,48,50,51,52,53,102$, $176,179,219$

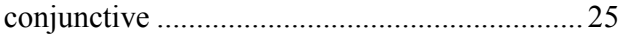

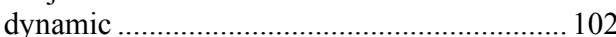

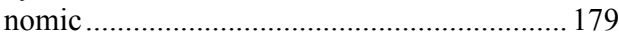

property ...................................................... 102

repeatable........24, 25, 29, 30, 38, 39, 40, 46, 176

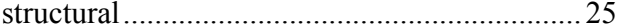

substantive ...................................................... 102

uninstantiated......................................... 24, 48

WaveTheory of Light.............................. 5, 237, 242

Wheeler, John ..................................... 77, 169, 274

Wuensch, Daniela ...................................... 224, 274

zero-point energy ............................................... 79 